\title{
52nd Annual Meeting of the Austrian Society of Surgery
}

\author{
Vienna, Austria, June 23-25, 2011 \\ Guest Editors: \\ Rudolf Roka, Gabriele Hastermann, Heidemarie Uher, Michael Vrba
}

\section{CONTENTS}

\section{VORTRÄGE}

Arbeitsgemeinschaft für Minimal Invasive

Chirurgie 1: SIL, Cholezystektomie . . . . . . . . 3

Chirurgische Forschung I: Immunologie,

Transplantation. ................ 5

Österreichische Gesellschaft für Mund-, Kiefer-

und Gesichtschirurgie: Interdisziplinäre

Diagnostik und Therapie kraniofazialer

Problemfälle ...................

3. Hauptsitzung - Funktionerhaltung und

Lebensqualität 1: Plastisch-rekonstruktive

Chirurgie. . . . . . . . . . . . . . . 11

Chirurgische Forschung II - Varia . . . . . . . . . 13

4. Hauptsitzung - Neue Techniken 1:

Interdisziplinäre Rundschau . . . . . . . . . . . . . . . . 15

Arbeitsgemeinschaft für Minimal

Invasive Chirurgie 2: SIL und Laparoskopie

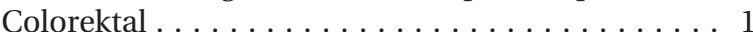

Arbeitsgemeinschaft für Hernienchirurgie

und Österreichische Gesellschaft für

Medizinische Videographie: Videositzung

Narbenhernie . . . . . . . . . . . . . . . . . 20

Chirurgische Forschung III: Onkologie,

Transplantation. . . . . . . . . . . . . . . . . 23

Österreichische Gesellschaft für Thorax-

und Herzchirurgie: Herzchirurgie - TAVI

und Neu Aortenklappenprothesen . . . . . . . . . . . 26

5. Hauptsitzung - Funktionserhaltung

und Lebensqualität $2 \ldots \ldots$. . . . . . . . . 29

6. Hauptsitzung - Onkologische

Viszeralchirurgie ................ 31

Arbeitsgemeinschaft für Minimal

Invasive Chirurgie: Varia . . . . . . . . . . . . . . . . . 35

Arbeitsgemeinschaft für Hernienchirurgie:

Leistenhernie . . . . . . . . . . . . . . . . 37

Chirurgische Forschung IV: Chirurgische

Innovationen. . . . . . . . . . . . . . . . . . 39

8. Hauptsitzung - Komplikationsmanagement

1: Herdsanierung. . . . . . . . . . . . . . . . . . 42

Österreichische Gesellschaft für Thorax-

und Herzchirurgie: Herzchirurgie -

Congenital und Varia . . . . . . . . . . . . . . . . . . 45

Österreichische Gesellschaft für Unfallchirurgie:

Varia . . . . . . . . . . . . . . . . . . . . . 49
9. Hauptsitzung - Neue Techniken 2: Zugänge . . . . 50

ACO/ASSO - Österreichische Gesellschaft für Chirurgische Onkologie: Multimodale Konzepte

und individualisierte Krebstherapie. . . . . . . . . . . 52

Arbeitsgemeinschaft für Qualitätssicherung

in der Chirurgie: Qualitätsverbessernde

Maßnahmen im Krankenhaus. . . . . . . . . . . . . . . 55

Österreichische Gesellschaft für Thoraxund Herzchirurgie: Herzchirurgie bei

Erwachsenen. . . . . . . . . . . . . . . . . . . 56

Österreichische Gesellschaft für Kinder-

und Jugendchirurgie: Technik, Varia . . . . . . . . . . 61

10. Hauptsitzung - Chirurgie und Recht . . . . . . . 64

Österreichische Gesellschaft für Handchirurgie:

Toxische Schädigungen an Hand

und Unterarm. . . . . . . . . . . . . . . . . . . . . 65

Österreichische Gesellschaft für Handchirurgie:

Verbrennungen an Hand und Unterarm . . . . . . . 65

Arbeitsgemeinschaft für Minimal Invasive

Chirurgie: Videositzung. . . . . . . . . . . . 66

Arbeitsgemeinschaft für Qualitätssicherung

in der Chirurgie: Varia. . . . . . . . . . . . . . . . . . 68

Österreichische Gesellschaft für Thorax-

und Herzchirurgie: Thoraxchirurgie - Varia . . . . 72

Österreichische Gesellschaft für Kinder-

und Jugendchirurgie: Neue Techniken

in der Kinder- und Jugendchirurgie . . . . . . . . . . 74

Österreichische Gesellschaft für

Adipositaschirurgie und Österreichische

Gesellschaft für Plastische, Ästhetische

und Rekonstruktive Chirurgie: Plastische

und Adipositaschirurgie I . . . . . . . . . . . . . . . . 77

Proktologie. . . . . . . . . . . . . . . . . . 81

12. Hauptsitzung - Komplikationsmanagement 2:

Beim chirurgischen Intensivpatienten. . . . . . . . 82

14. Hauptsitzung - Erweiterte Tumorchirurgie 2,

Chirurgie im hohen Senium . . . . . . . . . . 83

Österreichische Gesellschaft für

Kinder- und Jugendchirurgie:

Komplikationsmanagement. .

Österreichische Gesellschaft für

Adipositaschirurgie: Chirurgische Technik . . . . . 88

16. Hauptsitzung - Komplikationsmanagement 4:

Viszeralchirurgie ................. 92 


\section{2nd Annual Meeting of the Austrian Society of Surgery}

Österreichische Gesellschaft für Gefäßchirurgie: Aktuelles aus der arteriellen und venösen

Gefäßmedizin . . . . . . . . . . . . . . . . . . 94

18. Hauptsitzung - Berufsbild und chirurgische Karriere. . . . . . . . . . . . . . . . . . 97

Arbeitsgemeinschaft für Coloproctologie . . . . . . . 97

Österr. Ges. für Orthopädie und Österr. Ges.

für Plastische, Ästhetische und Rekonstruktive

Chirurgie: Weichteiltumore . . . . . . . . . . . . . 100

20. Hauptsitzung - Komplikationsmanagement 5:

Dehiszenz der ösophagogastrischen oder ösophagojejunalen Anastomose,

Ösophagusperforation. . . . . . . . . . . . . . . . 102

Herzchirurgie Thoraxchirurgie: Varia . . . . . . . . . 104

\section{POSTER}

Adipositaschirurgie. . . . . . . . . . . . . . . . . 115

Ausbildung. . . . . . . . . . . . . . . . . 116

Gefäßchirurgie . . . . . . . . . . . . . . . . 117

Handchirurgie . . . . . . . . . . . . . . . . . . 119

Kinderchirurgie . . . . . . . . . . . . . . 120
Arbeitsgemeinschaft für Chirurgische Endokrinologie 1: Incidentalom der Schilddrüse. Wann observieren? Wann operieren? Wie operieren?. . . . . . . . . . . . . . 107

Arbeitsgemeinschaft für Endoskopie in der Chirurgie: Neues und Bewährtes in der chirurgischen Endoskopie. . . . . . . . . . 108

22. Hauptsitzung - Komplikationsmanagement

6: Gallengangsverletzungen. . . . . . . . . . . 110

24. Hauptsitzung - Multimodale Konzepte. . . . . . 111 Arbeitsgemeinschaft für Chirurgische Endokrinologie 2: Primärer

Hyperparathyreoidismus . . . . . . . . . . . . . . 113

25. Hauptsitzung - Chirurgie in den Medien . . . . . 114

Komplikationsmanagement . . . . . . . . . . . . . 120

Krebstherapie... . . . . . . . . . . . . . . 122

Varia. . . . . . . . . . . . . . . . . . . . . . . . 128

Thoraxchirurgie . . . . . . . . . . . . . 130

Unfallchirurgie . . . . . . . . . . . . . . 131

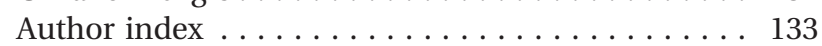

Für in der fortlaufenden Nummerierung fehlende Abstracts liegen keine Texte vor.

\section{Kongressorganisation:}

Wiener Medizinische Akademie, Bianca Theuer, Alserstraße 4, 1090 Wien

\section{Homepage:}

www.chirurgenkongress.at

\section{Kongresspräsident:}

Prim. Univ.-Prof. Dr. Rudolf Roka

\section{Kongress-Sekretäre:}

OA Dr. Gabriele Hastermann

Dr. Heidemarie Uher

OA Dr. Michael Vrba 


\section{VORTRÄGE}

\section{Arbeitsgemeinschaft für Minimal Invasive Chirurgie 1: SIL, Cholezystektomie}

\section{6}

\section{SILS Cholezystektomien (SILC) - Ergebnisse und kritische Analyse}

\section{F. Tomaselli, J. Huber, O. Gangl, W. Hofer, R. Függer \\ Department of Surgery, Linz, Austria}

Grundlagen. SILS zeigt uns eine Option, wodurch eine Reduktion des parietalen Traumas in der laparoskopischen Cholezystektomie erreicht werden könnte. Wir berichten über unsere Ergebnisse bei über 100 Patienten.

Methodik. Im Zeitraum 2/09 bis 1/11 wurden insgesamt 108 SILS Cholezystektomien (SILC) in standardisierter SILS Technik durchgeführt. Die ersten 67 SILC Patienten wurden unter dem Aspekt der Implementierung in einem matched paired Design analysiert.

Ergebnisse. Die Operationszeit in der matched pair Analyse (67/67) war länger bei SILC (median: 75 Minuten, 39-168 - konv. LCHE median 63 Minuten 29-165, $p=0,039)$ und zeigte auch bis dato keine Trendänderung (median: 73 Minuten, 32-168). Die postoperativen Schmerzen (in der matched pair analyse) gemessen mit VAS waren nach 24 Stunden (SILC median 3, range 0-8 vs. LC median 2, range $0-8 ; p=0,224)$ und auch nach 48 Stunden (SILC median 2, range 0-6 vs. LC median 2, range $0-8 ; p=0,571$ ) nicht unterschiedlich. Auch diesbezüglich imponierte keine wesentliche Änderung (median 3 , range 0-8) nach über 100 SILC Eingriffen. Insgesamt wurde SILC in $89,8 \%(97 / 108)$ erfolgreich beendet.

Schlussfolgerung. Die sichere Implementierung stellt eine der Grundvoraussetzungen für die notwendigen prospektiv randomisierten Studien dar. Ein Vorteil der Methodik kann momentan nicht bewiesen werden. Die Datenerhebung sollte durch Register unterstützt werden.

\section{7}

\section{Single incision laparoscopic cholecystectomy as the first choice approach in 300 patients}

\author{
J. Schirnhofer, W. Brunner, K. Pimpl, C. Mittermair, \\ C. Obrist, R. Frass, N. Waldstein, H. Weiss
}

Chirurgie, Salzburg, Austria
Background. During the last years single incision laparoscopy (SIL) has become popular for cholecystectomy (CHE). After having passed the learning curve of 15 SILcholecystectomies in selected patients we offered this approach to all patients.

Methods. From 08-2008 to 01-2011 we performed a number of 300 SIL-CHE without selection in all patients who were eligible for laparoscopy (mean age 56 years, mean BMI $26 \mathrm{~kg} / \mathrm{m}^{2}$ ). Different trocars were utilized as available. Different armamentarium was used. Additional trocars were inserted on demand. Perioperative parameters (operation time, complications, pain) were assessed. Data were prospectively collected in a data-base and analyzed.

Results. Eighty-seven percentage of all procedures could be completed without adding an extraumbilical trocar. In one patient conversion to open CHE was indicated for safety reasons. Operating time yielded in mean 52 minutes. The umbilical reconstruction was optimal in 94\%. Postoperative complications included two major complications (duodenal injury and bile duct laceration).

Conclusions. Initial expertise confirms the value of the novel transumbilical laparoscopic approach for cholecystectomy even at first choice. Surgical safety and outcomes has to remain uncompromised by adding one or more additional trocars.

\section{9}

\section{SIL-cholecystectomy: a teaching procedure?}

\section{K. Pimpl, W. Brunner, J. Schirnhofer, C. Mittermair, C. Obrist, N. Waldstein, R. Frass, H. Weiss}

Chirurgie, Salzburg, Austria

Background. While learning single incision transumbilical laparoscopy (SIL) the surgeon is constrained by the intersection of the instruments and the modified angles.

Methods. We report on the learning curve of SI-CHE performed by senior surgeons (A, $n=30$ ), fellows (B, $n=30$ ) or trainies (C, $n=30$ ) comparing total procedural time, intraoperative/postoperative complications, and length of incision. All surgeons passed wet-lab training before. The procedure took place in regular laparoscopic settings. Dissection and exposure was performed with the help of a suspension suture (cystopexia), one articulating instrument and a $5 \mathrm{~mm}$ optical device.

Results. All 90 procedures could be completed laparoscopically. Additional trocars were used for difficult dissection in $6 \%, 15 \%$ and $0 \%$ in groups $\mathrm{A}, \mathrm{B}, \mathrm{C}$, respectively. Total procedural time yielded in Mean $61 \mathrm{~min}, 84 \mathrm{~min}$, and $77 \mathrm{~min}$. Two complications required redo-laparoscopy (one in group A for bowel laceration and one in group $\mathrm{C}$ for hematoma). The other patients were on oral diet and started mobilization at the day of surgery. The follow-up was uneventful in all patients. Incisional length at the navel showed no difference between the groups. 
Conclusions. SIL-CHE can be proctored safely at least in selected cases and can be thereby regarded as a teaching procedure.

\section{0}

\section{Situs inversus totalis - first report on SILS-cholecystectomy}

\section{W. Mark, K. Kienzl-Wagner, M. Biebl, J. Pratschke}

Department of Operative Medicine, Innsbruck, Austria

Background. SILS is becoming to mature to the method of choice in uncomplicated cholecystectomy. In patients with situs inversus totalis laparoscopic surgery may be challenging and positioning of the surgeon has been discussed in one of the rare reports available.

Methods. We report on a 19 year old female patient $(\mathrm{BMI}=28)$ with symptomatic cholelithiasis and a known situs inversus totalis. There were no operations in the previous history and concomitant respiratory and cardiac anomalities. Therefore the indication for a SILS approach was set. After giving informed written consent the patient was operated in the French position. A single umbilical port and standard grasper, dissector and endobag were used.

Results. Upon laparoscopy mirrow-image anatomy was confirmed. Skeletalisation of Calot's triangle was feasible with ease and the procedure could be completed successfully without adding any additional trocar. The whole procedure took $65 \mathrm{~min}$ and blood loss was neglectable. After an uneventfull postoperative course the patient could be discharged on pod 3 .

Conclusions. This is the first report on SILS-cholecystectomy in situs inversus totalis.

\section{1}

\section{Erste Ergebnisse im Zentralkrankenhaus Bozen}

\section{G. Sitzmann, E. Brugger, F. Martin}

Abteilung für Allgemeinchirurgie, Zentralkrankenhaus Bozen, Bozen, Italy

Im Zeitraum von 13.12.2009 bis 19.10.2010 wurden an unserer Abteilung 24 Operationen von einem Operateur in SILS Technik durchgeführt: Davon waren 18 Cholezystektomien, 4 Appendektomien, 1 Anlage eines doppelläufiges Ileostomas und 1 Leberzysten Entdachung. Es wurden 9 Männer (27-71 Jahre; Median 48,44 Jahre) und 15 Frauen (16-72 Jahre; Median 43,73 Jahre) 18 mal programmiert und 6 mal im Notfall operiert. Die Operationsdauer betrug von $35 \mathrm{~min}$ bis $81 \mathrm{~min}$ (Median $49 \mathrm{~min}$ ). Postoperativ ergaben sich 1 Wundinfektion; 1 Choledocholithiasis mit nachfolgender ERCP; die VAS Werte (Schmerzskala) schwankten am 1. postoperativen Tag von 2-6, am 3. postoperativen Tag von 0-4 und zeigen damit vergleichbare Ergebnisse mit der konventionellen Laparoskopie. Im follow up nach 3 Monaten zeigten sich keine Nabelhernie, keine Schmerzen und zufriedenstellende kosmetische Ergebnisse. Die Technik ist sicher, zeigt verbesserte kosmetische Ergebnisse, erfordert grosse laparoskopische Erfahrung des Operateurs und hat technisches Entwicklungspotential für die Zukunft.

\section{2}

\section{SPIDER $^{\mathrm{TM}}$ - the "surgical" NOTES platform}

\section{J. Schirnhofer, W. Brunner, K. Pimpl, C. Mittermair, C. Obrist, R. Frass, N. Waldstein, H. Weiss}

Chirurgie, Salzburg, Austria

Background. A brand new operation platform is the single trocar SPIDER ${ }^{\mathrm{TM}}$ device allowing multiple flexible instruments to be used through one incision with increased degrees of freedom. We discuss the advantages and disadvantages of this innovative approach on a standard procedere such as the laparoscopic single incision cholecystectomy.

Methods. After wet-lab training and clinical immersion an initial number of three patients were operated for symptomatic cholecystolithiasis by using the first generation SPIDER ${ }^{\mathrm{TM}}$ decive (Transenterix). Perioperative parameters (operative time, complications, pain) were assessed. Data were prospectively collected in a data-base and analyzed.

Results. All procedures could be completed without adding an additional trocar. The overall procedural time yield 110, 113, and $127 \mathrm{~min}$ including adjustments for the team. The visualisation and handling of the device was advantageous without suspending sutures but strongly dependent on the size of the patient. Therefore, in two patients a GelPoint was used to obtain additional length. Dissecting, cutting, clip application, electrocautery, grasping, suturing and argon-beam vaporization was used sufficiently. No complication was noticed. Patient's satisfaction was optimal.

Conclusions. The SPIDER ${ }^{\mathrm{TM}}$ device allows to use high-flexible surgical instruments with the potential for a surgical NOTES platform. 


\section{3}

The SPIDER ${ }^{\mathrm{TM}}$ device: improvement of surgical performance?

\section{W. Brunner, J. Schirnhofer, R. Frass, N. Waldstein, K. Pimpl, C. Mittermair, C. Obrist, H. Weiss \\ KH Barmherzige Brüder, Salzburg, Austria}

Background. Laparoscopic surgery is the gold standard for several surgical procedures today. Since now only robotic systems allowed movements with seven degrees of freedom. The SPIDER ${ }^{\mathrm{TM}}$ device (Transenterics) is the first generation of a new class of flexible laparoscopic instruments. We herein describe the improvement of surgical perfomance based on our first experience with this new device.

Methods. In 10-2010 we performed 5 procedures with the SPIDER ${ }^{\mathrm{TM}}$ device via transumbilical approach based on our expertise of 909 single port laparoscopic surgery procedures.

Results. All operations (3 cholecystectomies, 1 TAPP, 1 rectopexy) could be completed in a single port technique. One cholecystectomy was performed only with the Spider device, in the other procedures we took a Gelpoint (Applied Medical) to introduce the device transumbilically. Dissection, clipping of the cystic duct and artery and removal was done via the flexible branches of the device. In the TAPP procedure only dissection was anatomically possible. In the rectopexy procedure dissection and suturing could be well done with the device.

Conclusions. The SPIDER ${ }^{\mathrm{TM}}$ device enables flexible moves with laparoscopic instruments and has the potental to improve the surgical perfomance. The shown difficulties with the length of the branches should leed to an optimized device for further use.

\section{Chirurgische Forschung I: Immunologie, Transplantation}

\section{7}

\section{Allergic comorbid diseases and histamine receptor expression (H1R, H2R) predispose for complicated courses of acute sigmoid diverticulitis}

B. von Rahden ${ }^{1}$, M. Grimm ${ }^{1}$, M. Jung ${ }^{1}$, D. Landmann ${ }^{1}$, S. Kircher ${ }^{2}$, M. Lazariotou ${ }^{1}$, C. Germer ${ }^{1}$

${ }^{1}$ Department of Surgery, University Wuerzburg, Wuerzburg, Germany; ${ }^{2}$ Department of Pathology, University Wuerzburg, Wuerzburg, Germany
Immunosuppression and steroids are significantly associated with complicated sigmoid diverticulitis. We hypothesize that allergies and histamine receptors predispose for complicated diverticulitis.

Expression of histamine and histamine receptors (H1R, H2R) were analyzed on protein and mRNA level in surgical specimen (complicated/uncomplicated diverticulitis). Results were correlated with clinical parameters.

The mean number of comorbid diseases per patient was $1.76 \pm 1.25$. Thirty-nine of 100 patients (39\%) exhibited allergic disposition. Comorbid diseases were significantly associated with complicated diverticulitis ( $p=0.027)$. Complicated diverticulitis was associated with H1R and H2R expression $(p=0.0304)$. An association of complicated diverticulitis with allergic preconditions was found $(\mathrm{OR}=3.2 ; 95 \% \mathrm{CI}: 1.3271-7.8239, p=0.0097)$. IF double-labelling showed a strong correlation of histamine expression on H1R and H2R expressing intestinal enterocytes (histamine/H1R, $\rho=0.841, p<0.0001$ and Histamine/H2R, $\rho=0.806, \quad p<0.0001)$. The results were confirmed on mRNA-level (RT-PCR, $p=0.009$ ).

Our findings suggest that allergic predisposition is another risk factor for complicated courses of sigmoid diverticulitis, and linked with histamine receptor expression. Similar to immunsuppressed patients and patients on steroids, early elective surgical resection should be considerer in patients with allergic comorbid diseases. Supportive therapies with antihistaminic drugs could be an option in addition to antibiotic standard pre-treatment.

\section{8}

Kann mittels Schockwellentherapie die Expression des VEGF im Anastomosengebiet einer enteralen Anastomose gesteigert und somit die Dehiszenzrate verringert werden? Eine experimentelle Studie im Rattenmodell

\section{W. Dabernig ${ }^{1}$, K. Emmanuel ${ }^{1}$, K. Schmid ${ }^{2}$, V. Maaß ${ }^{3}$, D. Öfner-Velano ${ }^{1}$ \\ ${ }^{1} \mathrm{PMU}$ Salzburg, LKH Salzburg, Abteilung für Chirurgie, Salzburg, Austria; ${ }^{2}$ Universitätsklinikum Essen, Institut fur Pathologie, Salzburg, Austria; ${ }^{3}$ PMU Salzburg, LKH Salzburg, Abteilung für Mikrobiologie, Salzburg, Austria}

Grundlagen. Die positive Wirkung von Schockwellen auf die Gewebedurchblutung wurde in der Literatur beschrieben und wurde von uns erstmalig an enteralen Anastomosen angewendet. Unterstützt durch die Schockwellentherapie (SWT) soll eine rasche Heilung einer Darmanastomose herbeigeführt und die Dehiszenzrate somit verringert werden. Die Literatur kennt bislang keine Publikation wo die SWT Behandlung an enteralen Anastomosen durchgeführt wurde.

Methodik. Nach Anlage einer Darmanastomose wurde jene im Rattenmodel intraoperativ einmalig mit 
SWT behandelt und eine Resektion der Anastomose in verschiedenen Zeitabständen durchgeführt (SW-Gruppe), die Kontrollgruppe blieb ohne Schockwellenapplikation (Ko-Gruppe). Die resezierten Anastomosen wurden histologisch aufgearbeitet, mittels eines Antikörper gegen VEGF behandelt und die VEGF Expression immunhistochemisch und mittels rtPCRs quantifiziert. Des Weiteren wurde der native Dünn.- und Dickdarm, welcher nicht anastomosiert wurde, mittels SWT behandelt und mit einer Kontrollgruppe, die ebenso unbehandelt blieb, verglichen.

Ergebnisse. Sowohl der nativ behandelte Dick.- und Dünndarm, als auch die Darmanastomosen der SWGruppe zeigten eine signifikant höhere Expression des VEGF nach einmaliger intraoperativer Behandlung gegenüber der Ko-Gruppe.

Schlussfolgerungen. Es werden Untersuchungen gefordert, die zur Verringerung einer Anastomosendehiszenz führen. Anhand dieser Studie wird gezeigt, dass Anastomosen, die mittels SWT behandelt wurden, eine erhöhte Expression von VEGF erreichen. Eine rasche Gefäßaussprossung und eine damit einhergehende erhöhte Durchblutung im Anastomosengebiet könnte die Anastomosendehiszenzrate verringern.

\section{9}

\section{Cell culture supernatants of peripheral blood mononuclear cells enhance wound healing in a murine full-thickness wound model}

\section{S. Hacker ${ }^{1,2}$, M. Mildner ${ }^{3}$, G. Werba ${ }^{2,4}$, T. Haider ${ }^{2,4}$, E. Tschachler ${ }^{3}$, M. Frey ${ }^{1}$, H. J. Ankersmit ${ }^{2,4}$}

${ }^{1}$ Department of Plastic and Reconstructive Surgery, Medical University of Vienna, Vienna, Austria; ${ }^{2}$ Christian Doppler Laboratory for Cardiac and Thoracic Diagnosis and Regeneration, Medical University of Vienna, Vienna, Austria; ${ }^{3}$ Department of Dermatology, Medical University of Vienna, Vienna, Austria; ${ }^{4}$ Department of Thoracic Surgery, Medical University of Vienna, Vienna, Austria

Background. Chronic skin ulcers are often resistant to common treatments. Previous studies have shown a positive effect on wound closure after topical treatment with growth factors. In this study we investigated whether cell culture supernatants (SN) of peripheral blood mononuclear cells (PBMC) induce enhanced wound healing in vivo. We further analyzed the effect of supernatants on human primary fibroblasts (FB), keratinocytes (KC) and endothelial cells (EC) in vitro.

Methods. Supernatants were harvested after 24 hours. Full-thickness dorsal punch biopsy wounds in C57BL/6j mice were treated with lyophilized cell culture supernatants for 4 days. The wound area including the surrounding tissue was excised on day 7. Tube formation assays, scratch assays, and cell cycle analyses were performed in vitro.
Results. Supernatants induced migration but not proliferation in FB. By contrast, supernatants induced migration and proliferation in KC and EC. Enhanced reduction of wound size was observed primarily during the first 3 days of treatment. H\&E staining revealed more advanced healing compared to control wounds. CD31 was strongly induced in wounds treated with SN.

Conclusions. Topical treatment of murine cutaneous wounds with PBMC-derived supernatants leads to early induction of wound closure. SN enhance migration and proliferation in human primary cells.

\section{0}

\section{Expression of peripheral node addressin (PNAd) in skin of human hand allografts: evidence of tertiary lymphoid organs}

\author{
T. Hautz ${ }^{1}$, B. Zelger ${ }^{2}$, I. Nasr ${ }^{3}$, G. Brandacher ${ }^{1,4}$, \\ L. Landin ${ }^{5}$, A. Weissenbacher ${ }^{1}$, P. Cavadas ${ }^{5}$, \\ R. Margreiter ${ }^{1}$, A. W. P. Lee ${ }^{4}$, J. Pratschke ${ }^{1}$, \\ F. G. Lakkis ${ }^{3}$, S. Schneeberger ${ }^{1,4}$
}

${ }^{1}$ Department of Visceral, Transplant and Thoracic Surgery, Medical University Innsbruck, Innsbruck, Austria;

${ }^{2}$ Department of Pathology, Innsbruck Medical University, Innsbruck, Austria; ${ }^{3}$ Division of Plastic Surgery, University of Pittsburgh Medical Center, Pittsburgh, USA; ${ }^{4}$ Department of Plastic and Reconstructive Surgery, Johns Hopkins University School of Medicine, Baltimore, USA; "La Fe" Hospital, Plastic and Reconstructive Surgery, Valencia, Spain

Background. We investigated the expression of PNAd in skin biopsies of human hand allografts for evidence of TLO after composite tissue allotransplantation.

Methods. One hundred and sixty-seven skin biopsies of 6 handtransplant recipients were assessed by HE-histology and immunohistochemistry (ABs for PNAd, CD3, CD4, CD8, CD20, C4d, CD68, LFA-1, ICAM-1, E-selectin, P-selectin, VE-cadherin, HLA-DR, Psoriasin, IDO and Foxp3). Levels of PNAd expression was assessed semiquantitatively (\% of PNAd+ vessels;staining intensity) and correlated with rejection grade, characterization of the infiltrate, expression of adhesion molecules and time after transplantation.

Results. Upon rejection, PNAd expression was increased in endothelial cells (grade 0: $0.24 \pm 0.48 v s$. all grades of rejection: $0.44 \pm 0.62$ ). Most often PNAd was only found in few vessels $(1-10 \%)$. PNAd staining intensity was increased the higher the grade of rejection. Intense PNAd-staining was associated with more CD4+ and CD8+ infiltrating T-cells, but less B-cells and macrophages, compared to mild PNAd staining intensity. Overall, PNAd expression correlated well with CD3+ cells and CD20+ B-cells. Poor correlation was found for expression of adhesion molecules, IDO and Foxp3. While PNAd expression was observed at all time-points after transplantation, staining intensity was slightly enhanced very early and late after transplantation. 
Conclusions. PNAd expression in endothelial cells is increased in skin biopsies of human hand allografts indicating presence of TLO. Further analysis is warranted to clarify the role of PNAd and TLOs in CTA.

\section{1}

\section{Effect of N-Acetylcysteine on acute allograft rejection after lung transplantation}

\section{B. V. Erne, W. Jungraithmayr, S. Arni, I. Inci, W. Weder}

University Hospital Zurich, Zurich, Switzerland

Background. We evaluate a protective effect of NAC against acute lung rejection.

Methods. Rat lung Tx was performed in four groups (each $n=7$ ). Donors and recipients of NAC group received NAC $150 \mathrm{mg} / \mathrm{kg}$ intraperitoneally before Tx and was repeated once daily until euthanasia. Control groups (CON) did not receive treatment. Euthanazation on day 1 or 5 post-Tx. Oxygenation $\left(\mathrm{PaO}_{2}\right)$ was measured before allograft recovery. Lung tissue was assessed by histology, immuno-histochemistry (IHC), immuno-fluorescence (IF), concentration of reduced glutathione level (GSH), and activated NF- $\kappa$ B levels.

Results. IHC showed significantly more CD68+ macrophages in CON5 compared to NAC5. No significant difference for $\mathrm{CD} 163+$ macrophages on day 5. CD3+ Tcells were significantly more frequent in NACl, but significantly less frequent in NAC5. IF yielded no significance between the goups for IL-4 and IL-12. GSH level was higher in NAC5 compared to CON5. In both groups on day $5, \mathrm{NF}-\kappa \mathrm{B}$ was significantly more activated compared to naive lung. Compared to CON5 group, NAC5 allografts had significantly less NF- $\kappa \mathrm{B}$ activation.

Conclusions. NAC attenuates acute pulmonary rejection by reduction of macrophage and T-cell-infiltration. This is intimately linked to a reduced action of the NF- $\kappa \mathrm{B}$ pro-inflammatory signalling pathway. NAC should be considered a promising substance in the prevention of acute rejection.

\section{2}

The influence of cold ischemia of pig hearts and vessels on mitochondrial function

\section{Wiedemann ${ }^{1,2}$, T. Schachner ${ }^{1}$, N. Bonaros ${ }^{1}$, O. Leberfing ${ }^{1}$, A. Kocher ${ }^{2}$, G. Laufer ${ }^{2}$, M. Grimm', A. Kuznetsov ${ }^{1}$}

${ }^{1}$ University Clinic of Cardiac Surgery, Innsbruck Medical University, Innsbruck, Austria; ${ }^{2}$ Department of Cardiac Surgery, Vienna Medical University, Vienna, Austria
In this study we aimed to investigate the effects of cold storage on mitochondrial function in pig myocardium and vessels, comparing also efficiency of HTK versus isotonic $\mathrm{NaCl}$ solution. Hearts, saphenous veins, internalmammary-artery and ascending aortas of male landrace pigs were harvested and stored in either NACL or HTK. After different times of cold ischemia mitochondrial function was determined by high resolution respirometry. No significant difference was found between mitochondrial respiratory capacities (maximal respiration rates) in control right and left ventricles $(32 \pm 8$ and $33 \pm 5$ with substrates for complex I glutamate/malate; and $48 \pm 14$ and $52 \pm 18 \mathrm{pmol} \mathrm{O}_{2}$ per s per mg wet weight with substrate for complexII succinate respectively). As compared with myocardium, mitochondrial respiration per mg in control vessels (aorta, arteries and veins) was more than 20 -folds lower. After $14 \mathrm{~h}$ of cold storage mitochondrial function estimated with either glutamate/malate or with succinate was significantly better preserved in HTK as compared with $\mathrm{NaCl}$ both in left and right ventricle. Similar superiority of HTK was observed also in aorta after $24 \mathrm{~h}$ of cold storage, whereas veins and arteries demonstrated stability of mitochondrial function in both solutions. High resolution respirometry analysis of mitochondrial function in permeabilized preparations is a suitable method for the assessment of cold ischemic injury in various tissues including heart and vessels

\section{3}

\section{Die inflammatorische Antwort des Spenderherzens nach einer Antithymozyten Globulin Therapie der hirntoten Maus}

\section{G. Pomper ${ }^{1,2}$, M. Hasun ${ }^{1}$, B. Thometich ${ }^{3}$, J. Kremer ${ }^{1}$, M. Inci ${ }^{1}$, K. Trescher ${ }^{1,4}$, A. O. Zuckermann ${ }^{5}$, B. K. Podesser ${ }^{1,4}$}

${ }^{1}$ Ludwig Boltzmann Cluster für kardiovaskuläre Forschung, Wien, Austria; ${ }^{2}$ Klinische Abteilung für Plastische und Rekonstruktive Chirurgie, Universitätsklinik für Chirurgie, Medizinische Universität Wien, Wien, Austria; ${ }^{3}$ Abteilung für Biomedizinische Forschung, Medizinische Universität Wien, Wien, Austria; ${ }^{4}$ Abteilung für Herzchirurgie, LK St. Pölten, St. Pölten, Austria; ${ }^{5}$ Klinische Abteilung für Herzchirurgie, Universitätsklinik für Chirurgie, Medizinische Universität Wien, Wien, Austria

Grundlagen. Die inflammatorische Reaktion in peripheren Organen nach Hirntod resultiert in einer vermehrten Abstossungsreaktion nach Transplantation. Wir haben in dem von uns etablierten Hirntodmodell der Maus die inflammatorische Antwort der Spenderherzen nach einer Antithymozyten Globulin (ATG) Therapie vor Organentnahme untersucht.

Methodik. Weibliche OF-1 Mäuse $(n=24)$ wurden in eine Hirntod- (BD) und eine Kontroll-Gruppe (Control) unterteilt. Jede dieser Gruppen wurde nochmals in eine 
ATG-Therapie-[ATG, BD ATG] und eine untherapierte Gruppe [BD, BD Control] randomisiert. Der Hirndruck wurde durch Inflation eines intrakraniell platzierten Ballonkatheters unter Elektroenzephalogramm (EEG) -Monitoring bis zum Eintritt des Hirntodes gesteigert. Das Hauptkriterium hierfür war ein Null-Linien-EEG. Am Ende einer 6-stündigen Beobachtungsphase wurde ATG (1 mg/kg KG) i.v. als Bolus appliziert und $45 \mathrm{~min}$ nach Beginn der Therapie wurden die Spenderherzen entnommen. Als proinflammatorische Marker im Myokard untersuchten wir IL-2 und IL-6 mittels ELISA.

Ergebnisse. Die Applikation von ATG vor Organentnahme führte $\mathrm{zu}$ einer signifikanten Reduktion der Expression von IL-6 (BD Control $v$ s. BD ATG: $p<0,05$ ) und von IL-2 (BD Control $v s$. BD ATG: $p<0,05$ ).

Schlussfolgerungen. Die Antithymozyten Globulin Therapie der hirntoten Maus zeigte eine Reduktion proinflammatorischer Zytokine im Myokard. Die Applikation von Antithymozyten Globulin beim hirntoten Organspender ist ein sinnvoller Therapieansatz zur Verbesserung der Organqualität vor Transplantation.

\section{4}

Donor treatment with tetrahydrobiopterin saves murine aortic allografts from chronic vasculopathy by attenuation of ischemia reperfusion injury

R. Oberhuber ${ }^{1}$, D. Bernhard ${ }^{2}$, B. Messner ${ }^{2}$, B. Cardini ${ }^{1}$, G. Riede ${ }^{1}$, W. Mark ${ }^{1}$, C. Steger ${ }^{3}$, G. Brandacher ${ }^{1}$, J. Pratschke ${ }^{1}$, K. Watschinger ${ }^{4}$, E. R. Werner ${ }^{4}$, M. Maglione ${ }^{1}$

${ }^{1}$ Department of Visceral, Transplant and Thoracic Surgery, Innsbruck Medical University, Innsbruck, Austria; ${ }^{2}$ University Clinic of Cardiac Surgery, Vienna Medical University, Vienna, Austria; ${ }^{3}$ Institute of Pathology, Innsbruck Medical University, Innsbruck, Austria; ${ }^{4}$ Division of Biological Chemistry, Biocenter, Innsbruck Medical University, Innsbruck, Austria

Chronic allograft vasculopathy (CAV) is a major obstacle to long term graft survival and is thought to be associated with ischemia reperfusion injury (IRI). Herein we analyzed whether tetrahydrobiopterin (H4B) attenuates CAV preventing IRI.

A fully MHC mismatched (BALB/c to C57BL/6) mouse cervical aortic transplantation model was used. Grafts were subjected to $24 \mathrm{~h}$ cold ischemia time (CIT). Donor animals received either H4B $(50 \mathrm{mg} / \mathrm{kg}$ b.w.) or saline. Aortas without CIT and syngeneic animals served as controls. Ten hours following reperfusion glutathione and HSP-70 Western blots and CD-31 immunohistochemistry were performed. Intimal hyperplasia was quantified by histopathology and immunohistochemistry 4 weeks following reperfusion.

Compared to controls CIT resulted in a significant reduction of glutathione tissue levels and increased
HSP-70 expression $(p<0.05)$. H4B-treatment resulted in significant restoration of glutathione levels and in a significant reduction of HSP-70 expression $(p<0.05)$. Furthermore, reduced CD-31 expression following CIT was attenuated by H4B. Four weeks following transplantation prominent intimal hyperplasia was observed in the untreated group but not following pre-treatment with $\mathrm{H} 4 \mathrm{~B}$ $(p<0.001)$, which, by contrast, was comparable with syngeneic controls and grafts without CIT.

These data suggest that IRI strongly correlates with CAV development. H4B might therefore represent a novel strategy to prevent CAV.

\section{Österreichische Gesellschaft für Mund-, Kiefer- und Gesichtschirurgie: Interdisziplinäre Diagnostik und Therapie kraniofazialer Problemfälle}

\section{8}

\section{Funktionserhaltung und Verbesserung der Lebensqualität durch rekonstruktive Maßnahmen in der MKG Chirurgie}

\section{S. A. Virnik ${ }^{1}$, A. Gaggl ${ }^{2}$, F. Chiari ${ }^{1}$}

${ }^{1}$ MKG Klagenfurt, Klagenfurt, Austria; ${ }^{2}$ Universität Salzburg, MKG Abteilung, Salzburg, Austria

Grundlagen. Mikrovaskuläre Transplantate zur Rekonstruktion in der MKG Chirurgie stellen einen wichtigen Teilaspekt dar um soziale, funktionelle und ästhetische Funktionen bei Patienten nach Tumor oder Traumata wieder herzustellen. Es wird im Rahmen des Kongresses mehrere Fälle vorgestellt die im Jahr 2011 auf der MKG Klagenfurt mittels mikrovaskulären Beckenkamm und Radialislappen rekonstruiert wurden.

Methodik. Es wurden an der MKG Klagenfurt in diesem Zeitraum zur Rekonstruktion von Gesichtsdefekten nach Tumorresektion ausschließlich mikrovaskuläre Beckenkammtransplantate und Radialislappen entnommen. Es werden drei dokumentierte Patientenfälle vorgestellt.

Schlussfolgerungen. Mikrovaskuläre Transplantate können erfolgreich zur Rekonstruktion von Alveolarkammdefekten verwendet werden. Das Transplantat kann individuell angepasst werden um anschließend eine Implantation mit festsitzender Versorgung durchzuführen. 


\section{9}

\section{Antikörper-assoziierte Osteonekrose der Kiefer unter Therapie mit Denosumab}

\section{G. Russmüller, R. Seemann, K. Pirklbauer, P. W. Pöschl, A. Wutzl, R. Ewers, C. Klug}

Universsitätsklinik für Mund-, Kiefer- und Gesichtschirurgie, AKH Wien - Universitätskliniken, Wien, Austria

Das Auftreten von Osteonekrosen der Kiefer ist ein Krankheitsbild, welches häufig bei Patienten auftritt, welche an schweren Knochenstoffwechselstörungen leiden oder mit antiresorptiven Medikamenten behandelt wurden. Sein Einführung der Bisphosphonate werden die Fälle von bisphosphonat-assoziierten Osteonekrosen der Kiefer immer häufiger. Als möglicher Ersatz für die Bisphosphonate wurde kürzlich der hier behandelte anti-RANK Antikörper Denosumab eingeführt.

Aufgrund der Behandlung von Patienten mit Denosumab im Rahmen klinischer Studien, wurden an unserer Institution erste Fälle von Denosumab-assoziierter Osteonekrose der Kiefer diagnostiziert und therapiert.

Erste Ergebnisse aus der Behandlung dieser neuen Entität der Osteonekrose zeigen Parallelen zur bisphosphonat-assoziierten Osteonekrose. Nach Absetzten der Therapie mit Denosumab und unter konservativer Therapie zeigte sich allerding nur eine partielle Besserung der klinischen Situation. Im Falle von chirurgischer Therapie zeigten sich bisher gute Ergebnisse.

Ähnlich zum Auftreten der bisphosphonat-assoziierten Osteonekrose der Kiefer könnten die kieferchirurgischen Zentren in Zukunft mit einer steigenden Zahl von Denosumab-assoziierten Ostenekrosen der Kiefer konfrontiert werden. Bei im Vergleich zur bisphosphonat-assoziierten Osteonekrose klinisch ähnlicher Ausprägung, aber grundsätzlich anderer Pharmakodynakik und -kinetik, muss bei diesem neuen Krankheitsbild nun der beste therapeutische Weg gefunden werden.

Wir berichten über die ersten Fälle von Osteonekrosen der Kiefer unter dem neuen antiresorptiven Medikament Denosumab und zeigen therapeutische Ergebnisse dieser neuen Form der Osteonekrose.

\section{0}

Pre-auricular hockey stick incision with retro-tragal development and tragus detachment for improved exposure of the condylar process

\section{Pau, M. Feichtinger, K. Reinbacher, P. Ivancic, H. Kärcher}

Department of Oral and Maxillofacial Surgery, Medical University of Graz, Graz, Austria
Background. We present a modification of the preauricular approach that improves the exposure of the condylar process. The incision resumes a hockey stick incision but its inferior part runs within the ear on the posterior face of the tragus. The tragal cartilage will be transsected together with the retrotragal skin and included in the anterior skin flap.

Methods. Between May 2009 and December 2010, 21 patients have been operated for various pathologies of the temporomandibular joint (TMJ).

Discussion. We find that a main disturbance in gaining access to the posterior edge of the TMJ is represented by anterior face of the EAC. This interferes with the visualization of the fractures and with the instruments, forcing the surgeon to an unfavourable obliquity while dissecting and while drilling. Our new approach improves the exposure of the TMJ combining the advantages of the hockey stick incision with those of the endoauricular and retroauricular incisions. Nevertheless, if compared with the standard preauricular approach, it adds any additional morbidity or increase of operative time.

Results. Every patient showed good aesthetical results, no infections or cartilage necrosis was observed in this survey.

Conclusions. This modification of the preauricular approach improves the exposure of the condylar head during open surgery of the TMJ ensuring easier manipulation of the joint and good cosmetic result.

\section{1}

\section{Komplikation einer elektiven, konventionell offen-chirurgischen Tracheotomie}

\section{P. Ivancic, G. Schultes, K. Reinbacher, H. Kärcher}

Department of Oral and Maxillofacial Surgery, Medical University of Graz, Graz, Austria

Die elektive konventionelle Tracheotomie nach Björk und Dukes ist bei einer Langzeitintubation aufgrund der Schwere der Grunderkrankung bzw. der zu erwartenden posttraumatischen und postoperativen Ödeme und Blutungen ein Routineeingriff und ist zur sicheren Gewährleistung der Atemwege eine absolute Indikation.

Bei unseren Patienten war eine Tumorresektion der Wange re., sowie radikale Neck dissection re., suprahyoidale Neck dissection li. und Rekonstruktion mit gestieltem M. pectoralis major Lappen geplant.

Bei der präoperativen PET-CT Schädel Hals Untersuchung wurde bereits eine knöcherne Struktur mit einer $6 \times 4 \times 3 \mathrm{~cm}$ Größe unklarer Genese beschrieben. Diese knöcherne Struktur hatte lt. CT einen engen Kontakt zur Schilddrüse, die sich als verkleinert darstellte. Der entgültige histologische Befund stand zum Zeitpunkt des Einreichens noch aus.

Intraoperativ bestätigte sich der CT Befund. Es zeigt sich ein vakuolisiertes, bienenwabenähnlich, mäßig kon- 
figuriertes Gerüst mit der oben beschriebenen Größe, welches diffus durchblutet war. Die Blutstillung konnte mit Koagulation nicht erreicht werden, weshalb mehrere Umstechungsnähte von Nöten waren.

Aufgrund des Tumors wurde die Trachea bereits massiv nach hinten und lateral, der Wirbelsäule aufliegend verschoben.

\section{2}

Interdisziplinäre Versorgung und Outcome komplexer Gesichtsschädelfrakturen mit frontobasaler Beteiligung

\section{R. Gassner ${ }^{1}$, S. Gerhard1, T. Ennemoser"1, M. A. Baubin', M. Rieger ${ }^{3}$, A. A. Obwegeser ${ }^{4}$}

${ }^{1}$ Universitätsklinik f. Mund-, Kiefer- und Gesichtschirurgie, Department f. Zahn-, Mund- und Kieferheilkunde u. Mund-, Kiefer- und Gesichtschirurgie, Medizinische Universität Innsbruck, Innsbruck, Austria; ${ }^{2}$ Universitätsklinik f. Anästhesie und Allgemeine Intensivmedizin, Department Notfallmedizin, Medizinische Universität Innsbruck, Innsbruck, Austria; ${ }^{3}$ Universitätsklinik f. Radiologie, Medizinische Universität Innsbruck, Innsbruck, Austria; ${ }^{4}$ Ärztliche Direktion, Landeskrankenhaus Innsbruck und Universitätsklinik f. Neurochirurgie, Medizinische Universität Innsbruck, Innsbruck, Austria

Ziel dieser Studie war die interdisziplinäre Analyse jener Patienten, die im Zeitraum Jänner 2003 bis Dezember 2008 an der Universitätsklinik Innsbruck mit komplexen frontobasalen Traumen und Gesichtsschädelfrakturen aufgenommen und zumindest von den Abteilungen Mund-, Kiefer- und Gesichtschirurgie und Neurochirurgie operativ behandelt wurden.

Insgesamt wurden im Beobachtungszeitraum 33 Patienten versorgt, die Geschlechts- und Altersverteilung, Unfallursache und -art wurden analysiert. Alle Patienten erlitten zumindest eine laterale Fraktur, in Kombination mit einer weiteren Fraktur des Os cribriforme oder der Ethmoidalzellen. Es handelte sich in allen Fällen um eine komplexe frontobasale Fraktur nach Sakas mit insgesamt 22 „large fractures“. Neben den Gesichtsschädelfrakturen und Läsionen im frontobasalen Viscerocranium, zeigten $36 \%$ der Patienten weitere Verletzungen an der Schädelbasis, bei $73 \%$ der Patienten waren zusätzliche Kalottenfrakturen zu erheben. Die mittlere Aufenthaltsdauer an der Klinik betrug $31 \pm 17$ Tage, davon verbrachten die Patienten $18 \pm 16$ Tage auf der Intensivstation.

Bei allen Patienten waren aufgrund der Komplexität der Verletzungen noch weitere Teams anderer Fachrichtungen involviert, um Synergien und Grenzen der einzelnen Fachdisziplinen effizient zu nützen. Diese Studie zeigt die aktuellen Perspektiven der Versorgung am Notfallort, die Behandlung und Abklärung im Schockraum als auch die weitere Patientenversorgung im Operationssaal und in der Nachsorge mit angewandten Vorgehensweisen und Algorithmen unserer Klinik.

\section{3}

Die Rolle der Funktionsdynamischen

Überbrückungsplatte (FDÜP) in der

Behandlung von entzündlich-destruktiven

Erkrankungen des Unterkiefers

(Osteomyelitis, Radioosteonekrose)

und komplizierten Unterkiefer-Frakturen

\section{S. Enzinger, J. Hachleiner, C. Krenkel, A. Gaggl}

Mund-, Kiefer- und Gesichtschirurgie Salzburg, Salzburg, Austria

Die von Krenkel zur Unterkieferrekonstruktion entwickelte FDÜP ist auch in der Behandlung entzündlicher Veränderungen des Unterkiefers (Osteomyelitis, Radioosteonekrose; 16 Patienten) sowie komplizierter UK-Frakturen (p.s. heilende Frakturen, Pseudoarthrosen, Trümmerfrakturen, Schußbrüche; 16 Patienten) erfolgreich zur Anwendung gekommen.

Die Formgebung der FDÜP lässt dosierte Kräfte zu, die die knöcherne Ausheilung fördern. Im Zeitraum 1990 bis 2010 wurden 32 Patienten aus diesem Spektrum mittels FDÜP therapiert und nachuntersucht.

In der Gruppe der entzündlich-destruktiven Erkrankungen des Unterkiefers kam es durch den Einsatz der FDÜP zusammen mit der Sanierung der entzündeten UKAreale bei 14 Patienten zu einer knöchernen Ausheilung mit Erhaltung der Sensibilität, notwendige Folgeoperationen konnten auf ein Minimum reduziert werden. In der Gruppe der komplizierten UK-Frakturen musste in einem Fall einer p.s. heilenden Unterkieferfraktur ein mikrovasculärer UK-Ersatz durchgeführt werden. Bei allen übrigen Patienten kam es zur Ausheilung.

Verglichen mit anderen Osteosyntheseverfahren sichert die FDÜP durch Positionierung der Osteosyntheseschrauben in sicherer Distanz zu den kompromittierten UK-Arealen und auch ohne Gefahr für den N.alv.inf. einen ungestörten Heilungsverlauf. Folgeoperationen können weitestgehend vermieden werden.

Durch dynamische Funktion bei Sicherung der Stabilität schafft die FDÜP in der Behandlung von entzündlich-destruktiven Prozessen und komplizierten Frakturen die Basis für eine problemlose knöcherne Ausheilung des Unterkiefers.

\section{4}

25 Jahre Erfahrung mit der KieferhöhlenEndothese in der Versorgung dislozierter Orbitabodenfrakturen

\section{Singh, J. Hachleitner, C. Krenkel, A. Gaggl}

Mund-, Kiefer- und Gesichtschirurgie Salzburg, Salzburg, Austria 
Die 1984 von Krenkel, Holzner und Lixl entwickelte Kieferhöhlen-Endothese ermöglicht bei ausgedehnten Orbitabodenfrakturen durch Retention der reponierten Fragmente in originärer Position in vielen Fällen eine Restitutio ad integrum.

Von 1985 bis 2010 wurden 503 Patienten mit Orbitabodenfrakturen mit diesem Verfahren therapiert. Nach Exploration des Orbitabodens über einen infraorbitalen Zugang wurde die Kieferhöhlen-Endothese über ein Fenster in der facialen Kieferhöhlenwand eingebracht, mit Kontrastmittel über ein nasales Drain gefüllt und 4 Wochen postoperativ nach Evakuation in Lokalanästhesie entfernt.

Alle Patienten wurden im Rahmen einer retrospektiven Studie bzgl. Persistenz von Doppelbildern, Auftreten eines En- oder Exophthalmus und von Sensibilitätsstörungen im Versorgungsgebiet des N. infraorbitalis nachuntersucht.

Im Rahmen der Spätkontrollen fand sich bei knapp einem Prozent der Patienten eine Diplopie in Primärstellung des Bulbus, bei 2,5\% eine solche im Gebrauchsblickfeld und bei $20 \%$ außerhalb desselben. Bei $6 \%$ der Patienten lagen die Hertelwerte außerhalb des Toleranzbereichs von $+2 /-2 \mathrm{~mm}$. Sensibilitätsstörungen bestanden bei knapp $20 \%$ der Patienten.

Verglichen mit rein überbrückenden Verfahren (Titangitter, Folien) können mit der Kieferhöhlen-Endothese alle knöchernen Fragmente zur Frakturheilung verwendet werden. Postoperative Korrekturen können einfach durchgeführt werden. Auf Dauer bleibt kein Fremdmaterial in situ.

Dieses operative Verfahren ermöglicht eine sichere und äußerst komplikationsarme anatomische Wiederherstellung des frakturierten Orbitabodens.

\section{Hauptsitzung - Funktionerhaltung und Lebensqualität 1: Plastisch- rekonstruktive Chirurgie}

\section{5}

\section{Synergie interventioneller Radiologie und plastischer Chirurgie bei der Behandlung von Patienten mit vaskulären Malformationen}

\section{K. Hirschl ${ }^{1}$, J. Roka-Palkovits ${ }^{1}$, J. Lammer ${ }^{2}$, H. Kubiena ${ }^{1}$, E. Frey ${ }^{3}$, M. Frey ${ }^{1}$}

${ }^{1}$ Abteilung für plastische und wiederherstellende Chirurgie AKH Wien, Wien, Austria; ${ }^{2}$ Abteilung für Interventionelle Radiologie AKH Wien, Wien, Austria; ${ }^{3}$ St. Anna Kinderspital, Wien, Austria

Grundlagen. Patienten mit gewissen Krankheitsbildern können nur in Zusammenarbeit von interventionellen Radiologen und Chirurgen behandelt werden. Dies sind Patienten mit diffusen venösen, lymphatischen und arterio-venösen Gefäßmalformationen. Dargestellt werden sollen Dauer der Symptomfreiheit bzw. Rezidivrate, Art des Sclerosans/Embolisat, Dosis und Intervall der Behandlung.

Methodik. Untersucht wurden 53 Patienten (156 Interventionen, $\mathrm{W}: \mathrm{M}=26: 27)$ im Beobachtungszeitraum 2001-2010. Grundlage bildete dabei die interdisziplinäre Erfassung gemäß der Klassifikation der ISSVA. Erfasst wurden demografischen Daten, läsionsspezifische, diagnostische und therapeutische Parameter (Art des Sclerosans/Embolisat, Dosis, Intervall). Anamnestische Daten wurden mittels Patientenfragebögen, SF 36 und DASH erhoben, eine Auswertung der spezifischen Datenbank erfolgte deskriptiv.

Ergebnisse. Die Läsionen verteilten sich wie folgt: AVM 11, VM 30, LM 11, LVM 1. Lokalisation: Kopf/Hals 24, Rumpf 3 OE 8, UE 18. Als Sclerosantien wurden Äthoxysclerol (57\%), Vibravenös (22\%), Bleomycin (5\%) und OK432 (16\%) eingesetzt. In der Regel wurde 3 Behandlungen im Abstand von 4-6 Wochen durchgeführt. Als Embolisate kamen Ethibloc, Contour, Coils und Glubran zum Einsatz. 15 Patienten wurden anschließend operiert.

Schlussfolgerungen. Bei Patienten mit diffusen venösen und lymphatischen bzw. arterio-venösen Malformationen ist ausschließlich ein kombiniertes Verfahren möglich, ein rein chirurgisch bzw. interventionelles Vorgehen ist bei vorliegen dieser Pathologie nicht indiziert.

\section{6}

\section{Rekonstruktion von Zungendefekten mit dem anterolateralen Oberschenkellappen}

\section{O. C. Aszmann, M. Paulhart, T. Rath, M. Frey}

Division of Plastic and Reconstructive Surgery, Vienna, Austria

Grundlagen. Relevante Zungenteildefekte führen zur Beeinträchtigungen der Schluck und Sprachfunktion und müssen rekonstruiert werden. Je grösser der Defekt desto substanzstärker muss der Lappen sein um der Restzunge Stabilität zu gewähren. Der ALT-Lappen hat sich wegen seiner substanzstärke und der minimalen Hebemorbidität auch bei grösseren Lappendimensionen für die Rekonstruktion von Defekten nach Tumorresektion im HNO Bereich etabliert. Hier berichten wir von der Anwendung dieses Lappens bei grossen Zungen und Zungengrunddefekten.

Methodik. In der Zeit von 2006-2010 kam bei fünf Patienten nach Resektion eines Plattenepithelkarzinoms an der Zunge und Zungengrund der ALT Lappen zum Einsatz. Drei von diesen 5 Patienten konnten für eine Nachuntersuchung gewonnen werden. Fotos und Videos wurden eingespielt. Die OP Berichte und Patientenakte wurden gesichtet und analysiert.

Ergebnisse. Es handelt sich durchwegs um Männer im durchschnittlichen Alter von 51 Jahren. (35-68) Bei allen 
Patienten war eine Hemiglossektomie notwendig mit mehr oder weniger grossen Anteilen den Mundbodens. Bei allen Patienten konnte ein primärer Verschluss der Heberegion erzielt werden und es zeigten sich hervorragende funktionelle Ergebnisses und gute Lebensqualität.

Schlussfolgerungen. Der ALT-Lappen wird bei Zungendefekten mit hervorragenden Ergebnissen zur Rekonstruktion eingesetzt. Der Hebedefekt des Lappens ist minimal, jedoch ist für einen erfolgreichen Einsatz dieses Lappen eine genaue Patientenselektion notwendig.

\section{7}

Maßnahmen zur Erhaltung und Wiederherstellung der mimischen Funktion wie auch der Lebensqualität bei älteren und tumorkranken Patienten mit Fazialisparese

\section{A. Hold, M. Michaelidou, I. Pona, E. Placheta, C. J. Tzou, M. Frey \\ MUW, AKH, Plastische und Rekonstruktive Chirurgie, Wien, Austria}

Grundlagen. Bei kompletter irreversibler Fazialisparese stellen dynamische Maßnahmen wie Muskeltransplantation oder Muskeltransposition eine gute Möglichkeit zur Wiederherstellung des Augenschlusses und des Lächelns dar. Bei Patienten, die aufgrund des Alters oder einer Tumorerkrankung keine Kandidaten für einen mikrochirurgischen Eingriff sind, wird der Muskeltransposition der Vorzug gegeben.

Methodik. Es wurden neunundzwanzig Patienten mit einer Temporalistransposition zur Verbesserung des Augenschlusses und acht Patienten mit einer Massetertransposition zur Wiederherstellung des Lächelns behandelt. Das durchschnittliche Alter betrug 53,4 \pm 16,7 Jahre (20 bis 85 Jahre). Die Ergebnisse wurden mittels eines 3D-Vieoanalysesystems dokumentiert und ausgewertet.

Ergebnisse. Die Weite des Lagophthalmus während des Augenschlusses betrug präoperativ durchschnittlich $9,59 \mathrm{~mm} \quad(\mathrm{SD} \pm 3,03 \mathrm{~mm})$, postoperativ nur mehr $4,33 \mathrm{~mm}(\mathrm{SD} \pm 2,68 \mathrm{~mm})$. Dadurch konnte ständiges Tränen und Trockenheitsgefühl des Auges bei allen Patienten drastisch reduziert werden. Die statische Asymmetrie des Mundwinkels verbesserte sich von präoperativ $14,17 \mathrm{~mm}$ $(\mathrm{SD} \pm 5,26 \mathrm{~mm})$ auf $5,38 \mathrm{~mm} \quad(\mathrm{SD} \pm 3,23 \mathrm{~mm})$. Somit erreichte die Bewegungsamplitude auf der rekonstruierten Seite $18 \%(S D \pm 19 \%)$ der Amplitude der gesunden Seite.

Schlussfolgerungen. Obwohl die funktionellen Ergebnisse der Muskeltransposition denen der freien Muskeltransplantation untergeordnet sind, stellt die Muskeltransposition ein wichtiges Werkzeug in der chirurgischen Therapie der Fazialisparese bei älteren und tumorkranken Patienten dar.

\section{8}

\section{Plastisch-Rekonstruktive Eingriffe nach Tumorresektion im Kopf-Halsbereich}

\section{O. C. Aszmann, M. Paulhart, I. Pona, T. Rath, M. Frey}

Division of Plastic and Reconstructive Surgery, Vienna, Austria

Grundlagen. Tumore in dieser Region stehen an der sechsthäufigsten Stelle der Statistik Austria. 2005 erkrankten in Österreich 843 Männer und 253 Frauen an diesen Tumoren. Histologisch handelt es sich vornehmlich um Plattenepithelkarzinome, welche primär einer chirurgischen Therapie zugänglich sind, jedoch auf Grund der Komplexität des Krankheitsverlaufes und der Region in einem interdisziplinären Tumorboard behandelt werden sollten.

Methodik. In den Jahren von 2001-2008 wurden an der Universitätsklinik für Chirurgie in Zusammenarbeit mit der Universitätsklinik für Hals-, Nasen- und Ohrenkrankheiten 69 Patienten behandelt, welche nach einer Tumorresektion eine Rekonstruktion mittels Lappenplastik benötigten. Die Ziele der Rekonstruktion waren kompetente Weichteildeckung und Rekonstruktion funktioneller Strukturen. Wir haben diese Patienten zu einer Nachuntersuchung eingeladen um das funktionelle Ergebnis und die Lebensqualität unter zu Hilfenahme eines internationalen validierten Fragebogens zu untersuchen.

Ergebnisse. Die am häufigsten eingesetzten Lappenplastiken waren der myokutane Pectoralis-major-Lappen, der radiale Vorderarmlappen, der ALT-Lappen und das freie Jejunumtransplantat. Wir mussten einen Lappenverlust und kleinere Komplikationen in $15 \%$ der Fälle verzeichnen.

Schlussfolgerungen. Die chirurgische Resektion von Plattenepithelkarzinomen ist sicherlich die beste Behandlungsstrategie. Diese Entscheidungsfindung erfordert ein interdisziplinäres Tumorboard. Die Methoden der plastischen Chirurgie haben in den letzten Jahren das funktionelle und ästhetische Ergebnis deutlich verbessert und sind aus diesem Patientengut nicht mehr wegzudenken.

\section{9}

\section{Ein kleiner Schnitt für den Chirurgen - eine „große“ Narbe für den Patienten}

\section{W. Michlits, M. Deutinger}

KA Rudolfstiftung, Abteilung für Plastische, Ästhetische und Rekonstruktive Chirurgie, Wien, Austria

Grundlagen. Jeder operativ tätige Mediziner ist früher oder später mit der Frage der richtigen Schnittführung bei 
Eingriffen konfrontiert. In der Traumatologie sind die Schnittführungen aufgrund der Wundverhältnisse teilweise vorgegeben. Bei einem geplanten Eingriff sollte jedoch stets eine optimale Schnittführung gewählt werden, um ansprechende und funktionell unauffällige Narben für den Patienten zu erzielen.

Methodik. Die Langer Spannungslinien, die weitgehend den „relaxed skin tension lines“ (RSTL) nach Borges entsprechen, stellen noch immer die Grundlage für die Wahl der Schnittführung dar. Anhand einiger Beispiele wollen wir Ihr Augenmerk auf diese Thematik lenken.

Ergebnisse. Neben der richtigen Ausrichtung der Schnittführung sind auch der Umgang mit dem Wundrand sowie die Nahttechnik entscheidende Faktoren, die das Erscheinungsbild einer Narbe wesentlich beeinflussen. Je feiner der Umgang mit dem Gewebe und je sauberer die Naht ausgeführt wird, desto besser sind die Ergebnisse.

Schlussfolgerungen. Für viele stellt die Schnittführung und der Umgang mit dem Gewebe eine gewohnte Banalität dar. Nichts desto trotz sollte man stets bemüht sein, auch bei kleinen Eingriffen Sorgfalt in der Planung und Durchführung walten zu lassen.

\section{0}

\section{Vaskuläre Malformationen an der Oberen Extremität - therapeutische Synergien und Grenzen}

\section{J. Roka-Palkovits ${ }^{1}$, T. Entner ${ }^{1}$, K. Hirschl ${ }^{1}$, H. Kubiena ${ }^{1}$, C. Plank ${ }^{2}$, J. Lammer ${ }^{2}$, M. Frey ${ }^{1}$ \\ ${ }^{1}$ Plastische und Rekonstruktive Chirurgie/Medizinische Universität Wien, Wien, Austria; ${ }^{2}$ Interventionelle Radiologie/Medizinische Universität Wien, Wien, Austria}

Vaskuläre Malformationen sind angeborene, chronische Erkrankungen des Gefäßsystems, wobei Ausprägung und Symptomatik individuell sehr unterschiedlich sein können.

Vaskuläre Malformationen an der oberen Extremität stellen aufgrund der hohen funktionellen Anforderungen eine oftmals massive und fortschreitende Beeinträchtigung für den Patienten dar.

Schmerzkontrolle und Funktionserhalt bzw. -verbesserung der Extremität zielen auf den Verbleib bzw. die Rückkehr des Betroffenen zu Alltags- und beruflichen Aktivitäten ab. Neben der chirurgischen Therapie haben sich insbesondere physikalische und interventionell-radiologische Methoden etabliert.

Zwischen Juni 2006 bis Dezember 2010 wurden an unserer Abteilung insgesamt 52 Patienten mit vaskulären Malformationen an der oberen Extremität vorstellig. Alle Patienten wurden interdisziplinär begutachtet. An 32 Patienten fanden operative Eingriffe statt, bei 31 Patienten kamen interventionell-radiologische Vorgehen zur Anwendung.
Die Entscheidung innerhalb eines interdisziplinären Settings ist das Fundament in der Behandlung von Patienten mit vaskulären Malformationen an der oberen Extremität. Der Entschluss zur Einleitung von Interventionen folgt diagnostischen und prognostischen Faktoren, die näher beleuchtet werden sollen. Nicht selten stoßen die therapeutischen Möglichkeiten dabei an ihre Grenzen.

\section{Chirurgische Forschung II - Varia}

\section{2}

\section{Recruitment of endothelial progenitor cells in chronic hind limb ischemia by extracorporeal shock wave therapy in rats}

\section{Tepeköylü ${ }^{1}$, F. S. Wang ${ }^{2}$, W. Schaden ${ }^{3}$, C. J. Wang ${ }^{4}$, M. Grimm ${ }^{1}$, J. Holfeld ${ }^{1}$}

${ }^{1}$ Department of Cardiothoracic Surgery, Medical University of Innsbruck, Innsbruck, Austria; ${ }^{2}$ Department of Medical Research, Chang Gung University College of Medicine, Chang Gung Memorial Hospital-Kaohsiung Medical Center, Kaohsiung, Taiwan; ${ }^{3}$ AUVA Trauma Center, Vienna, Austria; ${ }^{4}$ Department of Orthopedic Surgery, Chang Gung University College of Medicine, Chang Gung Memorial

Hospital-Kaohsiung, Kaohsiung, Taiwan

Background. Shock wave therapy (SWT) was shown to enhance recruitment of intravenously injected endothelial progenitor cells (EPC). It thereby improves angiogenesis in hind limb ischemia. We could show previously that direct epicardial SWT induces angiogenesis and improves left ventricular ejection fraction in ischemic heart failure in rats. We therefore hypothesized that injection of EPCs may not be necessary for muscle regeneration after SWT.

Methods. Five-week-old rats were subdivided in 3 groups: sham $(n=18)$, ischemic muscle with SWT (SWT-group, $n=18$ ) and without SWT (control, $n=18$ ). Hind limb ischemia was induced by ligation of femoral artery. Three weeks later SWT (300 impulses; $0.1 \mathrm{~mJ} / \mathrm{mm}^{2}$ ) was applied on the adductor muscle. Blood flow was measured by laser Doppler imaging. Muscle samples were analyzed by RT-PCR for angiogenetic factors and chemoattractants. FACS analysis of peripheral blood was performed for CD31/CD34 positive cells. Functional improvement was evaluated by walking analysis.

Results. Increased blood flow within the ischemic muscle and functional improvement were found after SWT. PCNA analysis revealed increased proliferation in the SWT-group. SDF-1 and VEGF were upregulated in the treatment group as shown by RT-PCR indicating the recruitment of EPCs. FACS analysis showed high numbers of CD31/CD34 positive cells in the SWT-group. 


\section{3}

\section{Injection of apoptotic peripheral blood mononuclear blood cells (PBMC) increases elastin expression in cardiac scar tissue after myocardial infarction}

\section{G. Werba ${ }^{1,2}$, M. Lichtenauer ${ }^{1}$, M. Mildner ${ }^{3}$, A. Baumgartner ${ }^{4}$, M. Hasun ${ }^{4}$, B. K. Podesser ${ }^{4}$, W. Klepetko ${ }^{1}$, H. J. Ankersmit ${ }^{1,2}$}

${ }^{1}$ Department of Surgery, Medical University of Vienna, Vienna, Austria; ${ }^{2}$ Christian Doppler Laboratory for Cardiac and Thoracic Diagnosis and Regeneration, Vienna, Austria; ${ }^{3}$ Department of Dermatology, Medical University of Vienna, Vienna, Austria; ${ }^{4}$ Ludwig Boltzmann Cluster for Cardiovascular Research, Vienna, Austria

Background. We have previously shown that injection of apoptotic cells improves left ventricular function after experimental myocardial infarction (MI) in rats.

Methods. Cell suspensions of apoptotic cells were injected intravenously or intramyocardially after experimental MI induced by coronary artery ligation in rats. Six weeks after induction of AMI the scar tissue was examined for the ratio of collagenous and elastic fibres. Cardiac function was quantified by echocardiography.

Results. Six weeks after MI animals treated with intravenous or intramyocardial administration of irradiated apoptotic PBMC, presented a remarkable accumulation of elastic fibers, mainly in the border zone of the MI. A planimetric analysis revealed that the fibrotic scar in apoptotic cell (IV and IM) injected rats was composed by $5.5 \% \pm 1.1$ and $8.9 \% \pm 2.2$ of elastic fibres compared to $0.2 \% \pm 0.1$ in controls and $2.9 \% \pm 0.2$ in viable injected animals ( $p<0.001$ vs. control, $n=10-12$ per group).

Conclusions. Injection of apoptotic cell suspensions resulted in attenuation of myocardial remodelling after experimental MI, preserved left ventricular function and altered the composition of cardiac scar tissue. The higher expression of elastic fibres could provide passive energy to cardiac scar tissue which results in prevention of ventricular remodelling.

\section{4}

Apoptotic peripheral blood mononuclear cells secrete pro-angiogenic factors that confer cytoprotection to cardiomyocytes

M. Lichtenauer ${ }^{1,2}$, M. Mildner ${ }^{3}$, K. Hoetzenecker ${ }^{1,2}$, S. Nickl ${ }^{1,2}$, G. Werba ${ }^{1,2}$, A. Mitterbauer ${ }^{1,2}$, M. Gyöngyösi ${ }^{4}$, W. Klepetko ${ }^{1}$, H. J. Ankersmit ${ }^{1,2}$

${ }^{1}$ Department of Surgery, Medical University of Vienna, Vienna, Austria; ${ }^{2}$ Christian Doppler Laboratory for Cardiac and Thoracic Diagnosis and Regeneration, Vienna, Austria; ${ }^{3}$ Department of Dermatology, Medical University of Vienna,
Vienna, Austria; ${ }^{4}$ Department of Cardiology, Medical University of Vienna, Vienna, Austria

Background. We could show previously that apoptotic peripheral blood mononuclear cells (PBMC) are able to reduce myocardial damage in a model of myocardial infarction (MI). We sought to examine the cardioprotective mechanisms that are induced by apoptotic cells and their secretome.

Methods. Cell culture supernatants derived from apoptotic PBMC (termed APOSEC) were collected and examined for pro-angiogenic and cytoprotective factors by ELISA and proteome membrane arrays. Cytoprotective effects of APOSEC were evaluated in cell starvation assays. The induction of pro-survival signalling-cascades in primary human cardiomyocytes was determined by western blot analysis and mebrane arrays.

Results. Induction of apoptosis in PBMC increased the secretion of many pro-angiogenic cytokines and growth factors ( $p<0.05$ and 0.01 compared to controls). Exposure of cardiac myocytes to the secretome of apoptotic cells triggered the activation of pro-survival signalling-cascades (AKT, p38 MAPK, Erk1/2, CREB, c-Jun), increased anti-apoptotic gene products (Bcl-2, BAG1) and protected cardiomyocytes against starvation induced cell death in a time and dose dependent manner in vitro.

Conclusions. The supernatant of apoptotic PBMC contains a broad spectrum of pro-angiogenic and cytoprotective factors that induces the activation of pro-survival signalling-cascades in cardiomyocytes.

\section{5}

Secretome of apoptotic peripheral blood cells (APOSEC) confers cytoprotection to cardiomyocytes and inhibits tissue remodelling after acute myocardial infarction

\section{Lichtenauer ${ }^{1,2}$, M. Mildner ${ }^{3}$, K. Hoetzenecker ${ }^{1,2}$, S. Hacker ${ }^{1,2}$, M. Zimmermann ${ }^{1,2}$, B. K. Podesser ${ }^{4}$, W. Sipos ${ }^{5}$, E. Berényi ${ }^{6}$, E. Tschachler ${ }^{3}$, M. Gyöngyösi $^{7}$, W. Klepetko ${ }^{1}$, H. J. Ankersmit ${ }^{1,2}$ \\ ${ }^{1}$ Department of Surgery, Medical University of Vienna, Vienna, Austria; ${ }^{2}$ Christian Doppler Laboratory for Cardiac and Thoracic Diagnosis and Regeneration, Vienna, Austria; ${ }^{3}$ Department of Dermatology, Medical University of Vienna, Vienna, Austria; ${ }^{4}$ Ludwig Boltzmann Cluster for Cardiovascular Research, Vienna, Austria; ${ }^{5}$ Clinical Department for Farm Animals and Herd Management, University of Veterinary Medicine, Vienna, Austria; ${ }^{6}$ Department of Biomedical Laboratory and Imaging Science, Faculty of Medicine, University of Debrecen, Debrecen, Hungary; ${ }^{7}$ Department of Cardiology, Medical University of Vienna, Vienna, Austria}

Background. Our previous observation that injection of apoptotic peripheral white blood cells was able to re- 
store long-term cardiac function after myocardial infarction (MI) prompted us to study the effect of soluble factors derived from apoptotic cells on ventricular remodelling.

Methods. Cell culture supernatants derived from apoptotic cells (APOSEC) were collected and injected as a single dose intravenously after myocardial infarction in an experimental MI rat model and in a porcine closed chest reperfused MI model. Magnetic resonance imaging was used to quantitate cardiac function.

Results. Intravenous administration of APOSEC resulted in a reduction of scar tissue formation in both MI models. In the porcine reperfused MI model APOSEC led to higher values of ejection fraction $(57.0 \% v s .40 .5 \%$, $p<0.01)$, a better cardiac output $(4.0$ vs. $2.4 \mathrm{l} / \mathrm{min}$., $p<0.001)$ and a reduced extent of infarction size $(12.6 \%$ vs. $6.9 \%, p<0.02)$ as determined by MRI. Administration of APOSEC in the rat MI model caused increased presence of macrophages in the infarcted myocardium.

Conclusions. Infusion of culture supernatant of apoptotic cells attenuated myocardial remodelling in both models of experimental MI.

\section{6}

\section{Recipient and donor body mass index correlate with initial kidney graft function}

\section{A. Weißenbacher, M. Jara, M. Biebl, C. Bösmüller, J. Pratschke, R. Öllinger}

Department of Visceral, Transplant and Thoracic Surgery, Center of Operative Medicine, Innsbruck Medical University, Innsbruck, Austria

Background. We investigated whether donor and/or recipient BMI correlate with the occurrence of delayed graft function (DGF).

Methods. Retrospective analysis of 1132 consecutive cadaveric kidney transplants between 01/2000 and $12 / 2009$. Recipients/donors were devided into four groups according to their BMI $(<20,20-25,>25-30$, $>30$ ). DGF was defined as the requirement for more than one dialysis within the first post-transplant week.

Results. Overall DGF rate was $32.1 \%$, mean BMI was $23.75(\mathrm{SD} \pm 3.8)$ for all recipients and $24.68(\mathrm{SD} \pm 3.6)$ for all donors (median age $44.0 ; 40.3 \%$ female). DGF rate was $25.2 \%, 29.8 \%, 40.9 \%$ and $52.6 \%$ in recipients with a $\mathrm{BMI}<20, \quad 20-25, \quad>25-30$ and $>30$ respectively $(p<0.0001)$. Donor $\mathrm{BMI}<20,20-25,>25-30$ and $>30$ resulted in a DGF rate of $22.5 \%, 31.0 \%, 37.3 \%$ and $51.2 \%$ $(p<0.0001)$. Multivariate analysis revealed overweight in the recipient, besides cold ischemia and anastomosis time, as an independent risk factor for DGF $(p<0.0001)$.

Conclusions. Recipient and donor BMI as well closely correlates with the incidence of DGF. Awareness thereof should have an impact on peri- and post transplant measures in renal transplant recipients.
4. Hauptsitzung - Neue Techniken 1: Interdisziplinäre Rundschau

\section{8}

\section{Bionische Rekonstruktion der schwer geschädigten oberen Extremität- ein neuer Ansatz in der rekonstruktiven Extremitätenchirurgie}

\section{O. C. Aszmann ${ }^{1}$, M. Herczeg ${ }^{2}$, H. Dietl ${ }^{3}$, M. Frey ${ }^{1}$}

${ }^{1}$ Division of Plastic and Reconstructive Surgery, Vienna, Austria; ${ }^{2}$ Universitätsklinik für Physikalische Therapie und Rehabilitation, Vienna, Austria; ${ }^{3}$ Otto Bock Healthcare Products $\mathrm{GmbH}$, Vienna, Austria

Manche Verletzungen der oberen Extremität führen zwar nicht notwendigerweise zu einem physikalischen Verlust der Hand oder des Armes, aber ziehen einen mehr oder weniger großen Funktionsverlust nach sich. Dies gilt z.b. für massive Plexusverletzungen aber auch für elektrische Verbrennungen- um nur zwei wesentliche Verletzungsmuster $\mathrm{zu}$ nennen. Falls die biologische Rekonstruktion $\mathrm{zu}$ einem unbefriedigenden Ergebnis führt kann die bionische Rekonstruktion möglicherweise eine sinnvolle Alternative darstellen und wesentliche Extremitätenfunktion wiederherstellen.

Exemplarisch wollen wir die Möglichkeiten der bionischen Rekonstruktion an Hand einiger Fallbeispiele darstellen und die Grenzen und Möglichkeiten dieses neuen spannenden Ansatzes erörtern und diskutieren. Die Bandbreite geht von Doppelarmamputierten bis hin zu Patienten nach mutilierenden Extremitätenverletzungen welche nach eingehender Aufklärung und Versorgung mit „Hybridlösungen“ sekundär amputiert wurden und schließlich mit modernster Technik eine Arm/Handfunktion wiedererlangt haben.

Anhand von Videos und Interviews werden Langzeitergebnisse dargestellt und die Möglichkeiten der bionischen Rekonstruktion dargestellt.

\section{9}

Interdisziplinäres operatives Management vaskulärer Malformationen

\author{
H. Kubiena ${ }^{1}$, K. Hirschl ${ }^{1}$, J. Roka-Palkovits ${ }^{1}$, M. Frey ${ }^{1}$, \\ J. Lammer ${ }^{2}$, E. Frey ${ }^{3}$ \\ ${ }^{1}$ Klin.Abt.f.Plastische und Rekonstruktive Chirurgie, Wien, \\ Austria; ${ }^{2}$ Klin.Abt.f.Kardiovaskuläre und Interventionelle \\ Radiologie, Wien, Austria; ${ }^{3}$ St.Anna Kinderspital, Wien, Austria
}

Grundlagen. Fehldifferenzierungen im Rahmen der Vaskulogenese führen zu vaskulären Malformationen, 
einer Vielfalt unterschiedlicher abnormer Komponenten des Gefäßbettes. Eine interdisziplinäre Indikationsstellung begründet ein operatives Vorgehen für dessen erfolgreiche Umsetzung die Berücksichtigung bestimmter Prinzipien empfehlenswert ist.

Methodik. In den letzten 10 Jahren wurden 70 Patienten mit vaskulären Malformationen operiert. Davon fanden sich 20 Läsionen im Kopf-/Halsbereich, 7 im Stammbereich, 23 an der oberen und 20 an der unteren Extremität. In den meisten Fällen (60) war nach Resektion ein Direktverschluß möglich, in 5 Fällen kamen lokale Lappenplastiken, und in 5 Fällen eine Spalthauttransplantation zur Anwendung.

Ergebnisse. Indikationen waren: rezidivierende Thrombophlebitiden, Ulzeration, Expansion, ästhetischer Beeinträchtigung, Schmerzen sowie Funktionseinschränkung. In 5 Fällen war ein Folgeeingriff erforderlich. In einem Fall wurde infolge eines Fortschreitens der Erkrankung eine weitere Resektion indiziert. Die Rezidivrate beträgt $2 \%$. In keinem der Fälle war eine Erythrozytensubstitution erforderlich.

Schlussfolgerungen. Ein operatives Vorgehen in der Behandlung vaskulärer Malformationen beruht auf einer strukturierten Indikationsstellung: Morphologie der jeweiligen Läsion (fokal versus diffus) sowie spezifische Charakteristika (Schobinger Stadium, mikro- versus makrozystisch) sind dabei von Bedeutung. Das chirurgische Vorgehen kann neben speziellen Techniken der Blutsperre und Lagerung, durch den Einsatz von präoperativen interventionellen Verfahren, die Verwendung spezieller Instrumente und mittels spezifischer Präparationstechniken optimiert werden.

\section{0}

\section{Erste Erfahrungen mit direkter Stimulation des Nervus pudendus bei Stuhlinkontinenz}

\section{Lechner ${ }^{1}$, H. Rosen ${ }^{2}$}

${ }^{1} \mathrm{KH}$ der Barmherzigen Schwestern Wien, Wien, Austria; ${ }^{2} \mathrm{KH}$ göttlicher Heiland Wien, Wien, Austria

Grundlagen. Die sakrale Neuromodulation ist ein etabliertes Therapiekonzept in der Behandlung der Stuhlinkontinenz. Zuletzt wurde in einigen Arbeitsgruppen eine direkte Stimulation des Nervus pudendus an seiner Austrittsstelle aus dem Alcock'schen Kanal durchgeführt.

Methodik. Ziel unserer Studie war zu objektivieren ob durch direkte Stimulation des Nervus pudendus bessere Ergebnisse als durch Stimulation der Nervenwurzel im Rahmen einer SNM erzielt werden können.

Bei 8 Patienten wurde eine SNM-Elektrode in gewohnter Weise implantiert. Zusätzlich wurde unter rektal-digitaler Kontrolle durch eine zweite Elektrode der Nervus pudendus direkt stimuliert. Die subchronische Testphase erfolgte analog dem Vorgehen bei SNM.
Ergebnisse. Von 8 Patienten bei denen eine direkte Stimulation des Nervus pudendus durchgeführt wurde, konnte bei 6 Patienten die subchronische Testphase erfolgreich abgeschlossen werden. Bei einer Patientin wurde wegen fehlendem Erfolg die Elektrode explantiert, ein Patient befindet sich noch in der Testphase.

Schlussfolgerungen. Bei den für die direkte Pudendusstimulation ausgewählten Patienten handelte es sich durchwegs um komplexe Problemstellungen. Trotzdem konnte die Implantantionsrate im Vergleich zur SNM verbessert werden $(75 \%$ bei Pudendusstimulation im Vergleich $\mathrm{zu} 70 \%$ bei SNM). Sollten sich die ersten Erfahrungen mit der direkten pudendus-stimulation (PS) in einem groesseren Kollektiv reproduzieren lassen, wuerde dies eine wesentliche Erweiterung des Behandlungsspektrums bedeuten

\section{1}

\section{Ultraschalluntersützte Thrombolyse mit dem EKOS-System zur Behandlung der tiefen Venenthrombose}

\section{J. Grommes ${ }^{1}$, A. Greiner ${ }^{1}$, A. H. Mahnken ${ }^{2}$, M. Jacobs ${ }^{1}$, C. H. Wittens ${ }^{1}$ \\ ${ }^{1}$ Europäisches Gefäßzentrum Aachen-Maastricht Klinik für Gefäßchirurgie der RWTH Aachen, Aachen, Germany; ${ }^{2}$ Klinik für Radiologische Diagnostik und Interventionelle Radiologie der RWTH Aachen, Aachen, Germany}

Grundlagen. $25 \%$ aller iliofemoraler Thrombosen entwickeln ein postthrombotisches Syndrom innerhalb von 2 Jahren. Die pharmako-mechanische Thrombolyse zur Behandlung der TVT stellt eine neue Therapieoption zur Thrombusentfernung dar, um akute Symptome und Langzeitschäden einer femoroiliacalen Thrombose $\mathrm{zu}$ reduzieren.

Methodik. Das EKOS ${ }^{\circledR}$ EndoWave $^{\mathrm{TM}}$ System kombiniert einen Infusionskatheter mit mehreren seitlichen Öffnungen zur kontinuierlichen Medikamenteninfusion und einen Hochfrequenzultraschall mit niedriger Energie. Seit 1/2009 wurden 23 Patienten mit einer schweren iliofemoralen oder cavalen Thrombose mit der kathetergesteuerten Thrombolyse behandelt. Nach Platzieren des Katheters im zentralen Thrombusbereich wurde die Lyse mit einem $5 \mathrm{mg}$ rtPA-Bolus begonnen und $1 \mathrm{mg} / \mathrm{h} \mathrm{rtPA}$ und 1000 I.E.Heparin fortgeführt. Angiographische Kontrollen wurden nach 12 Stunden durchgeführt.

Ergebnisse. In 21 von 23 Fällen war die Thrombolyse erfolgreich. Lungenembolien traten während der Therapie nicht auf. Komplikationen waren Hb-relevante Blutung $(n=1)$ und Reverschlüsse $(n=3)$. Frühreverschlüsse wurden durch residuelle venöse Obstruktion $(n=3)$ und HIT II Syndrom $(n=1)$ verursacht. Im weiteren Follow-up zeigten sich keine Reverschlüsse. In 6 Fällen wurden verbliebenen Stenosen mit PTA und Stent erfolgreich behandelt.

Schlussfolgerungen. Die pharmako-mechanische Thrombolyse mit EKOS ${ }^{\circledR}$ stellt eine sichere Methode zur 
Behandlung der TVT dar. Verbliebene Stenosen sollten mit Angioplastie und Stent versorgt werden. Weitere prospektiv randomisierte Studien sind notwendig, um die pharmako-mechanische Thrombolyse im Langzeitverlauf zu beurteilen.

\section{2}

Applied tissue engineering and reconstructive medicine in challenging hernia repair and treatment of complex abdominal wall defects

A. H. Petter-Puchner ${ }^{1}$, R. H. Fortelny ${ }^{2}$, S. Gruber-Blum ${ }^{2}$, K. S. Glaser ${ }^{2}$, H. Redl ${ }^{1}$

${ }^{1}$ Ludwig Boltzmann Institute for Experimental and Clinical Traumatology, Vienna, Austria; ${ }^{2}$ Wilhelminenspital, Vienna, Austria

Background. Increasing numbers of very complex repair procedures, e.g. "loss of domain" or closure of open abdomen in ICU patients, demand dedicated implants and fixation devices. Although a variety of new biomaterials and fixation strategies were introduced in the past decade there is still room for improvement. Tissue integration, infection and biocompatibility remain key issues. Scepticism is fuelled by mixed results from clinical and experimental trials. Therefore, research has turned to tissue engineering and reconstructive medicine in order to adopt possible advantages for hernia repair.

Methods. Our study group investigates scaffolds and implants combining favorable properties of biologic matrices (low susceptibilty for infection) or synthetic meshes (reliable and fast integration) with the potential of amniotic membrane or stem cells. Amniotic membrane effectively enhances integration and reduces adhesion formation. To provide the best enviroment for these new implants, different supportive techniques are currently assessed, including fibrin sealant coatings or non invasive tissue preparation.

Results. Excellent results have been obtained with tissue engineered implants derived from first experiments in our lab. Amniotic was readily available for clinical use.

Conclusions. In the presentation the most promising strategies will be discussed with focus on the clinical relevance and the current literature in the field.

\section{3}

\section{Ex vivo Lungenperfusion - der Probelauf vor der Transplantation}

A. Slama ${ }^{1}$, A. Scheed ${ }^{1}$, K. Hötzenecker ${ }^{1}$, W. Schmid ${ }^{2}$, B. Urbanek ${ }^{3}$, G. Lang', W. Klepetko ${ }^{1}$, C. Aigner ${ }^{1}$
${ }^{1}$ Department of Thoracic Surgery, Vienna, Austria;

${ }^{2}$ Department of Anaesthesia and Intensive Care, Vienna, Austria; ${ }^{3}$ Department of Anaesthesia and Intenisve Care, Vienna, Austria

Background. Ex-vivo lung perfusion (EVLP) provides the opportunity to evaluate and optimise unacceptable donor-lungs prior to transplantation. We report on initial clinical results of a prospective study of lung-transplantation after ex-vivo-perfusion.

Methods. All donor-lungs who are deemed unacceptable by our standard-criteria and meet the inclusion-criteria are evaluated with EVLP using acellular Steen-Solution for 2-4 hours. Lungs showing a arteriovenous $\Delta \mathrm{PaO}_{2}>350 \mathrm{mmHg}$ with stable or improving functional-parameters are accepted for transplantation.

Results. From 3/2010 to 1/2011 12 lungs were evaluated ex-vivo. Mean donor-age was 43 years (range 16-58). Mean donor $\mathrm{PaO}_{2} / \mathrm{FiO}_{2}$ was $207 \pm 49 \mathrm{mmHg}$. Four lungs, all with trauma-history did not improve. 8 lungs improved to $\Delta \mathrm{PaO}_{2}>350 \mathrm{mmHg}$ (mean $\mathrm{PaO}_{2} 472 \pm 32 \mathrm{mmHg}$ ). These lungs were transplanted with a mean total-ischemic-time of $584 \pm 56 \mathrm{~min}$. Mean recipient age was 47 years (range 18-66). Diagnoses were fibrosis $(n=3)$, COPD $(n=3), \mathrm{CF}(n=1), \mathrm{SPH}(n=1) .7$ recipients had a PGD score 0 at $24 \mathrm{~h}$ posttransplant. The patient with SPH was left on ECMO postoperatively for hemodynamic reasons showing good lung-function. Median intubation-time was 2 days. Thirty-day-mortality was $0 \%$.

Conclusions. EVLP is a safe and effective method to evaluate and optimise nonacceptable donor-lungs. In the setting of an already very liberal use of marginal donororgans we were able to transplant 8 additional lungs which would not have been used otherwise with excellent short-term-results.

\section{4}

\section{Kathetergestützte}

Aortenklappenimplantation (TAVI) erfolgreiche Implementierung durch interdisziplinäre Kooperation

\section{S. Pfeiffer ${ }^{1}$, G. Santarpino ${ }^{1}$, M. Herold ${ }^{2}$, M. Hinzmann ${ }^{2}$, J. Jessl $^{3}$, M. Pauschinger ${ }^{3}$, T. Fischlein ${ }^{1}$ \\ ${ }^{1}$ Klinikum Nürnberg - Klinik für Herzchirurgie, Nürnberg, Germany; ${ }^{2}$ Klinikum Nürnberg - Klinik für Anästhesiologie, Nürnberg, Germany; ${ }^{3}$ Klinikum Nürnberg - Klinik für Kardiologie, Nürnberg, Germany}

Grundlagen. Nach Markteinführung wurde in Nürnberg ein TAVI Programm etabliert. Aufgrund der transapikalen (TA) und transfemoralen (TF) Implantationsmöglichkeit wurde dafür die „Edwards SAPIEN“ Prothese ausgewählt.

Methodik. Die SAPIEN wird entweder transfemoral (Retroflex) oder transapikal (Ascendra) realisiert. Auswahl der Hochrisikopatienten und Durchführung der Implan- 
tation erfolgt durch ein multidisziplinäres Team (Herzchirurgie, Kardiologie, Anästhesie). Peripherer Gefäßstatus und Komorbiditätsprofil bestimmen den Zugangsweg.

Ergebnisse. Bis Januar 2010 wurde bei 51 Patienten (Pat) die SAPIEN implantiert: TA 27 (53\%), TF 24 (47\%). LogEuroScore war 25.58 (TA 25.9, TF 25.2, $p=$ NS), mittleres Alter 81.2 Jahre für TA und 83.3 für TF $(p=\mathrm{NS})$ (52.4-92.3). TA wurde über eine linksanterolaterale Minithorakotomie durchgeführt, für TF wurde die Leistenarterie über eine limitierte Inzision chirurgisch freigelegt. Alle Pat wurden ohne HLM operiert, keine Konversion zur offenen OP.1 Pat ( $2 \%)$ benötigte eine zweite Klappe („valve in a valve“) bei ausgeprägter paravalvulärer Leckage. Paravalvuläre AI $(<2)$ war bei $60 \%$ der Pat. Bei 1 TF Pat $(4,2 \%)$ benötigte eine Gefäßprothese im Bereich der A.fem. aufgrund einer lokalen Dissektion. 30-Tage Mortalität war 5,8\% (1 (3.7\%) TA und 2 (8.5\%) TF, $p=0.216$ ).

Schlussfolgerungen. Für chirurgische Hochrisikopatienten ist die TAVI inzwischen bevorzugte Alternative zum offenen Aortenklappenersatz. Der multidisziplinäre Ansatz ist ein wichtiger Faktor zum Erfolg.

\section{5}

Minimally invasive mitral valve reconstruction on the fibrillating heart an attractive therapeutic alternative for high-risk patients

\section{J. Kilo, H. B. Hangler, K. Stifter, A. Heinz, M. Grimm, L. Müller}

Medical University of Innsbruck, Innsbruck, Austria

Reoperative mitral valve surgery is associated with high operative risk. An aortic valve prosthesis (AVP) can severely impair visualization to the mitral valve, so that these patients are often denied surgery. Furthermore, patients with severely calcified aorta are usually considered inoperable.

Mitral valve repair without aortic crossclamping on the fibrillating heart may be an attractive surgical option for these extremely high-risk patients.

We report the series of seven patients undergoing mitral valve surgery via a right-sided minithoracotomy without aortic crossclamping on the fibrillating heart. Three patients had an AVP in situ, three patients underwent CABG before, two patient presented with porcelain aorta. Reconstruction was possible in 6 patients, one patient, who underwent mitral valve repair plus CABG before, received mitral valve replacement. Cannulation for cardiopulmonary bypass was performed femorally in three and via the axillary artery in four patients.

No fatalities were observed. One patient required rethoracotomy for bleeding. One patient suffered from ischemic embolism to the leg due to the arterial pressure line. The postoperative course was uneventful in all other cases. No patient presented with significant residual mitral insufficiency in control echocardiography.

Mitral valve reconstruction via a right-sided minithoracotomy is an attractive surgical option in high-risk reoperative settings.

\section{Arbeitsgemeinschaft für Minimal Invasive Chirurgie 2: SIL und Laparoskopie Colorektal}

\section{6}

\section{Ergebnisse nach laparoskopischen und offenen kolorektalen Resektionen bei malignen und benignen Grunderkrankungen}

\section{F. Schneider, C. Wimmer, G. Bischof}

St. Josef Krankenhaus, Wien, Austria

Grundlagen. Ziel der Studie ist es Vor- und Nachteile der laparoskopischen Colonchirurgie gegenüber offenen kolorektalen Eingriffen zu evaluieren.

Methodik. Im Zeitraum 04/2005 bis 12/2010 wurde im St. Josef KH an 382 Patienten ein kolorektaler Eingriff vorgenommen, wovon 149 (39\%) Patienten laparoskopisch und 233 (61\%) Patienten offen reseziert wurden. Der Anteil maligner Erkrankungen liegt mit 238 Patienten bei $62,3 \%$ wovon $73,1 \%$ der Patienten (=174) offen und $26,9 \%$ der Patienten (=64) laparoskopisch operiert wurden.

Die UICC Stadienverteilung ist in Tab. 1 dargestellt.

Ergebnisse. Das Durchschnittsalter bei offenen Resektionen liegt bei 70,3 $(n=233)$, bei lap. Eingriffen bei $67,2(n=149)$. Das 5 Jahres Überleben liegt bei $73,1 \%$ bei Patienten (maligne Grunderkrankung, $n=26$ ) mit lap. Eingriffen und bei 74,4\% bei der offenen Gruppe

\begin{tabular}{|c|c|c|c|}
\hline $\begin{array}{l}\text { UICC } \\
\text { Stadium }\end{array}$ & Offen & Laparoskopisch & Gesamt \\
\hline I & $32(64 \%)$ & $18^{1}(36 \%)$ & 50 \\
\hline$\|$ & $52(73,2 \%)$ & $19^{2}(26,8 \%)$ & 71 \\
\hline III & $65(73 \%)$ & $24^{3}(27 \%)$ & 89 \\
\hline IV & $12(80 \%)$ & $3(20 \%)$ & 15 \\
\hline Gesamt & 161 & 64 & $225^{4}$ \\
\hline \multicolumn{4}{|c|}{$\begin{array}{l}1 \text { 1 } 1 \times \text { Umstieg lap }- \text { offen. } \\
{ }^{2} 3 \times \text { Umstieg lap }- \text { offen. } \\
{ }^{3} 2 \times \text { Umstieg lap }- \text { offen. } \\
{ }^{4} \text { Abweichung zur Gesamtzahl }(n=238) \text { durch } 13 \text { nicht. klassifizierbare } \\
\text { Tumore. }\end{array}$} \\
\hline
\end{tabular}


$(n=39)$. Die Gesamtzahl der Patienten im Langzeit Follow-up beträgt 65 ( $n=78$; entspr. 83,3\%). Die Morbiditätsrate $30 \mathrm{~d}$ post $\mathrm{OP}$ lag bei $18 \%$ offen und $11,4 \%$ laparoskopisch.

Schlussfolgerungen. Offene und laparoskopische Eingriffe können sowohl bei benigner als auch maligner Indikation mit niedriger Mortalitäts- und Morbiditätsrate durchgeführt werden. Komplikationen nach Resektionen sind in beiden Fällen selten.

\section{7}

\section{Outcome of 130 single incision laparoscopic colorectal procedures}

\author{
W. Brunner, J. Schirnhofer, R. Frass, N. Waldstein, \\ K. Pimpl, C. Mittermair, C. Obrist, H. Weiss \\ KH Barmherzige Brüder, Salzburg, Austria
}

Background. Single-access laparoscopic surgery should offer reduction of the surgical trauma without compromising surgical outcome. Herein we describe our technique of single port laparoscopic colorectal procedures.

Methods. Between 09-2008 and 12-2011 we performed 130 laparoscopic colorectal procedures (Mean \pm SD age $66 \pm 13$ years, BMI $26 \pm 5 \mathrm{~kg} / \mathrm{m}^{2}$ ) out of a total number of 896 patients undergoing single access surgery for neoplasma or polyps (65). The entire operation was carried out transumbilically or via the ileostomy position in the French position using three trocars or a single-port system.

Results. All but eight procedures could be completed without adding additional trocars, one was converted to open. Anastomosis was completed by means of stapling (118) or suturing (8), 4 patients underwent rectopexy without colonic resection. Operation time lasted (Mean) 140 min. Specimen retrieval was carried out trough the port site, transrectal (2) or transvaginal (2). Specimen length yielded (Mean) $27 \mathrm{~cm}$, number of harvested lymphnodes was 16 (Mean).

The fast-track concept was respected. Reoperation was necessary for anastomotic leakage (8), and others (3). Patients were discharged on day (Mean \pm SD) $8 \pm 10$. Umbilical hernia (2) and mild wound infections (5) were observed during follow-up.

Conclusions. The novel technique of SIL colorectal procedures does not compromise surgical outcome but allows for optimal postoperative cosmesis.

\section{8}

\section{SILS Colonchirurgie - mitten in der Lernkurve?}

\section{J. Huber, F. Tomaselli, O. Gangl, W. Hofer, R. Függer}

Department of Surgery, Linz, Austria
Grundlagen. Innovationen, wie SILS zeigen uns vielversprechende Optionen, wodurch eine Reduktion des parietalen Traumas in der laparoskopischen Chirurgie erreicht werden könnte. Wir berichten über unsere ersten Ergebnisse in der SILS Colonchirurgie.

Methodik. Im Zeitraum 12/2009 bis $7 / 2010$ wurden 19 Patienten (14 Frauen und 5 Männer, 47-83 Lj, i. M. 67, $1 \mathrm{Lj}$ ) von einem definiertem OP Team in standardisierter SILS Technik (GelPort ${ }^{\circledR}$ laparoscopic system, (C) 2010 Applied Medical Resources Corporation, konventionelle MIC Instrumente) operiert: 1 Ileocäcalresektion, 10 Hemicoleoctomien rechts und 8 Sigmaresektionen. Die durchschnittliche OP Dauer betrug im Durschschnitt 130,6 Minuten (min.: 51 und max.: 244 min.).

Ergebnisse. 15 Colonresektionen konnten in SILS Technik beendet werden (2 OP's: 2 additv ports, 2 OP's Laparotomie). Bei allen 11 malignen Indikationen konnten R0 Resektionen bei adäquater Lymphadenektomie (min.: 10, max.: 20, i.M.: 13,2 Lymphknoten) erreicht werden.

Die postoperative Verweildauer betrug im Schnitt 7,5 Tage. Eine Re-Operation (Mesenterialtorsion) und 3 Minor Komplikationen (2 subkutane Wundinfekte und ein passagerer Pneumothorax) wurden beobachtet.

Schlussfolgerungen. Bei bereits etablierter laparoskopischer Colonchirurgie kann SILS ohne aufwendige technische und logistische Innovationen implementiert werden. Ein Vorteil der Methodik kann momentan auf Grund der fehlenden, randomisierten Studien nicht bewiesen werden. Die Datenerhebung sollte durch nationale bzw. internationale Register unterstützt werden.

\section{9}

Single incision laparosopic colectomy: the search for the optimal speciment retrieval site

\section{J. Schirnhofer, W. Brunner, K. Pimpl, C. Mittermair, C. Obrist, N. Waldstein, R. Frass, H. Weiss}

Chirurgie, Salzburg, Austria

Background. The aim of the single incision laposcopic (SIL) colectomy is to reduce the surgical trauma. Since dissection can be carried out through a small incision, specimen retrieval finally requires enlargement of the surgical orifice. Hybride techniques combining the transvaginal or transanal approach are described. Herein we discuss our experience and considerations of specimen retrieval utilizing three different routes.

Methods. Small skin incisions in 130 transumbilical SIL colorectal resections did not allow specimen retrieval without enlargement of the incision. As an alternative we used the transvaginal (2 patients) and transrectal (2 patients) route, respectively. Intra- and postoperative complications, pain (VAS, medication) and patient's satisfaction were evaluated. 
Results. Changing the retrieval site from the navel to the vagina or anus requires additional technical support. This adds more than 20 minutes to the overall procedural time. No complication was observed. Squeezing of the specimen is of no concern since all are secured within a retrieval bag. Patients report on almost no pain after the transvaginal or transrectal approach.

Conclusions. Depending on the size of specimen and gender of the patient the retrieval is feasible through the umbilicus, the vagina or the rectum. Pros and Cons are under debate.

\section{0}

\section{Wound complications in transumbilical single incision laparoscopy: analysis of 970 consecutive procedures}

\section{H. Weiss, W. Brunner, J. Schirnhofer, R. Frass, N. Waldstein, K. Pimpl, C. Mittermair, C. Obrist}

KH Barmherzige Brüder, Salzburg, Austria

Background. Single incision laparoscopy (SIL) has revolutionized the technical developments in minimal invasive surgery. Although evidence for procedural feasibility and safety is growing some criticism for the proven benefits other than better cosmesis and in particular concerns about impaired umbilical wound healing remain.

Methods. From 10/2008 to 01/2011 we performed a total of 970 SIL-procedures. In all patients (Mean \pm SD age $56 \pm 16$ years, BMI $26 \pm 10 \mathrm{~kg} / \mathrm{m}^{2}$, sex $436 \mathrm{f} / 467 \mathrm{~m}$ ) the umbilical diameter, skin and fascia incisions as well as preexisting fascial defects were documented. Perioperative parameters were documented.

Results. Three hundred and fifteen 315 cholecystecomies, 356 TAPP, 132 colorectal, 78 appendectomies, 15 liver resections, 13 fundoplications, 9 small bowel resections, 5 pancreas resections, 5 bariatric procedures, 3 adrenalectomies, 38 others were performed. Preexisting fascial defects were observed in $45 \%$ of patients. An incisional length of $2.9 \mathrm{~cm}$ and $3.0 \mathrm{~cm}$ was necessary for skin and fascia, respectively, to insert the instruments and retrieve any specimen. In the follow-up of up to 25 months, wound infection was found in $2.3 \%$ (all but one treated conservatively) and incisional hernia in $0.9 \%$ of patients, respectively. Interrupted stitches for fascial closure were disadvantageous.

Conclusions. Concerns of increased risk for wound complications after transumbilical SIL procedures are gratuitous.

\section{1}

\section{Single Incision Laparoscopic Surgery (SILS) - unsere Erfahrungen mit Indikationstellung und Komplikationen}

\author{
E. Laimer ${ }^{1}$, A. Klaus ${ }^{2}$, A. Perathoner ${ }^{1}$, G. Mühlmann ${ }^{1}$, \\ M. Biebl ${ }^{1}$, J. Pratschke ${ }^{1}$, R. Mittermair ${ }^{1}$ \\ ${ }^{1}$ Univ. Klinik für Viszeral-, Transplantations- und \\ Thoraxchirurgie, Innsbruck, Austria; ${ }^{2}$ Krankenhaus \\ Barmherzige Schwestern, Wien, Austria
}

Grundlagen. Im September 2008 wurde an unserer Abteilung die erste SIL-Cholezystektomie durchgeführt. Mittlerweile zählt diese Operation zu unseren Standardoperationen. Nach einer anfänglichen Lernkurve haben wir dann das operative Spektrum auf weitere Operationsgebiete ausgedehnt.

Methodik. Zwischen September 2008 und Jänner 2011 wurden 245 Patienten $(\mathrm{w}=165, \mathrm{~m}=80)$ an unserer Abteilung mit der SILS-Technik operiert. Berichtet wird über die transumbilikale SILS Cholezystektomie $(n=140)$, Appendektomie $(n=76)$, TEP-Hernienchirurgie $(n=24)$ und Sleeve-Gastrektomie $(n=5)$.

Ergebnisse. Die Indikationsstellung erfolgte ohne spezielle Patientenselektion, sondern richtete sich nach der Anwesenheit der SILS-operierenden Chirurgen. Bezüglich der Komplikationen trat bei 8 Patienten nach SILS Cholezystektomie eine intraabdominelle Retention auf, wobei drei Patienten operativ revidiert wurden. Nach SILS Appendektomie entwickelten 6 Patienten eine intraabdominelle Retention, bei drei Patienten erfolgte ein Reeingriff, 4 Patienten nach SILS-TEP entwickelten ein Rezidiv.

Schlussfolgerungen. Die transumbilikale SILS-Technik ist nach einer kurzen Eingewöhnungszeit eine sichere und gut durchzuführende Operationsmethode.

\section{Arbeitsgemeinschaft für Hernienchirurgie und Österreichische Gesellschaft für Medizinische Videographie: Videositzung Narbenhernie}

\section{2}

Open retromuscular mesh augmentation with fibrin sealant mesh fixation in incisional hernia repair

R. H. Fortelny ${ }^{1,2}$, A. H. Petter-Puchner ${ }^{1,2}$, H. RedI ${ }^{2}$, K. Glaser ${ }^{1}$ 
${ }^{1}$ Wilhelminenspital, Department of General, Visceral and Oncological Surgery, Vienna, Austria; ${ }^{2}$ Ludwig Boltzmann Institute for Experimental and Clinical Traumatology, Vienna, Austria

Background. Reinforcement of the abdominal wall with alloplastic mesh material in incisional hernia repair is well established. To avoid dislocation and migration of the mesh in the early postoperative period, fixation is recommended. The most common used technique is the suture fixation. However, there seems to be a correlation between postoperative pain and kind of mesh fixation.

Methods. This video demonstrates a retromuscular mesh implantation using a polypropylene-polyglecaprone 25 composite mesh (Ultrapro ${ }^{\mathbb{B}}$ ). The mesh is fixed to the posterior rectus sheet using $5 \mathrm{ml}$ fibrin sealant (Tissucol $^{\circledR}$ ) by spray-application.

Results. Using this kind of fixation technique we detected no dislocation or migration of the mesh in our patient.

Conclusions. To avoid the risk of lesion to vessels, nerves and intestinal organs by sutures for mesh fixation complete atraumatic fixation by fibrin sealant achieves a safe, elastic and broad fixation of the mesh to the posterior sheet and avoids mesh dislocation or migration in the early postoperative period.

\section{3}

\section{Fibrin sealing by laparoscopic spray system for mesh fixation in IPOM-repair}

\author{
R. H. Fortelny ${ }^{1,2}$, A. H. Petter-Puchner ${ }^{1,2}$, H. Redl ${ }^{2}$, \\ K. Glaser ${ }^{1}$ \\ ${ }^{1}$ Wilhelminenspital, Department of General, Visceral and \\ Oncological Surgery, Vienna, Austria; ${ }^{2}$ Ludwig Boltzmann \\ Institute for Experimental and Clinical Traumatology, \\ Vienna, Austria
}

Background. In the majority of cases of chronic pain observed after repair of groin and ventral hernias are linked to nerve injury from penetrating mesh fixation devices, such as staples, tacks or sutures. Tissue sealants have been proposed to provide atraumatic mesh fixation until fibrous incorporation of the implant into the abdominal wall occurs. In several preclinical studies of hernioplasty, mesh fixation with fibrin sealant (FS) was proven to be feasible, and efficient in terms of fixation strength.

Methods. A special designed laparoscopic spray applicator achieves a precise area and angle controlled coverage of the fibrin sealant.

Results. First own clinical results suggest that mesh fixation with FS using this laparoscopic spray system in IPOM-procedure may effectively reduce the number of additional traumatic fixation devices e.g. sutures and the rate of complications.

Conclusions. FS in combination with sutures for mesh fixation in IPOM repair seems to obtain good results in terms of tensile strength, mesh incorporation and at least improvement of patient comfort.

\section{4}

\section{Unsere Erfahrungen mit IPOM Technik bei Narben-, Nabel- u. epigastrischen Hernien}

\section{A. Wiegele, E. Dapunt, H. Marsoner, U. Pandini, R. Pizzinini}

Chirurgie KH Bruneck, Bruneck, Italy

Grundlagen. Angeregt durch vielversprechende Literaturangaben haben auch wir vor sechs Jahren begonnen, Narbenbrüche sowie Nabelbrüche und epigastrische Hernien laparoskopisch mit der IntraPeritonealen OnlayMesh-Plastik (IPOM) zu versorgen.

Methodik. Wir haben in der Zeit von 2004 bis 2010 115 Patienten mit der IPOM-Plastik behandelt. Dabei handelte es sich um 49 Frauen und 66 Männer im Alter von 21-82 Jahren (Durchschnittsalter 58.1 Jahre). 43 Patienten hatten eine Nabelhernie, 42 eine Narbenhernie, 22 eine epigastrische Hernie, 5 eine Trokarhernie und ein Patient hatte eine Hernia Spigelii. Der Durchmesser der Bruchpforten betrug $2-12 \mathrm{~cm}$. Die Netzgröße betrug $10 \times 15 \mathrm{~cm}$ bis $20 \times 30 \mathrm{~cm}$. Die Netzfixation erfolgte mittels verschiedener gerade zur Verfügung stehender Fixationssysteme.

Ergebnisse. Intraoperativ gab es keine Komplikationen. An Minor-Komplikationen resultierten postoperativ Schmerzen $(n=6)$, Hämatome $(n=2)$, Nabelinfektion $(n=1)$ und eine Nabelfistel, an Major-Komplikationen ein Ileus und eine Darmwandläsion. Nach einem Follow-up von 1-43 Monaten (durchschnittlich 18.1 Monate) hatten wir zwei Hernienrezidive zu verzeichnen.

Schlussfolgerungen. Die Inzidenz der Narbenhernien beträgt $10-20 \%$. Die konventionelle Narbenhernienchirurgie hat ihrerseits eine Rezidivrate von 37-63\%. Spannungsfreie Reparaturen haben diese auf $10 \%$ gesenkt. Erste Analysen zeigen Vorteile in der Rate lokaler Wundkomplikationen, Rezidive und Rekonvaleszenz. Unsere vergleichsweise guten Ergebnisse sind durch Langzeitbeobachtung zu verifizieren.

\section{5}

Kulissenplastik als Erweiterung der bekannten Komponentenseparation in der Behandlung von Narbenhernien

\section{H. Klapper, R. Klug}

Landesklinikum Horn, Horn, Austria

Die hohe Rezidivrate bei direkt verschlossenen Narbenhernien hat zur Entwicklung zahlteicher Verschluss- 
verfahren von Mittellinienbrüchen geführt, welche in der Mehrzahl den Einsatz von Kunststoffnetzen erfordern. Die am häufigsten erwähnte Methode zum spannungsfreien Bauchdeckenverschluss ohne Verwendung von Fremdmaterial ist die sogenannte Komponentenseparation nach Ramirez. Die Kulissenplastik stellt eine Erweiterung dieser Methode dar. Mit ihr ist der nahezu spannungsfreie Verschluss noch grösserer Narbenhernien möglich.

Im Vortrag wird die Methode vorgestellt und Ergebnisse werden präsentiert.

\section{6}

Minimal invasive Komponentenseparation nach Rosen - erste klinische Erfahrungen versprechen deutliche Vorteile gegenüber der Ramirez-Plastik

\section{F. Mayer, M. Lechner, M. Baschata, J. Hutter, D. Öfner-Velano}

PMU Paracelsus Medizinische Privatuniversität, Salzburg, Austria

Grundlagen. Die netzverstärkte Reparation einer Narbenhernie der Mittellinie kann nicht selten auf Grund einer ausgeprägten Retraktion der Rektusmuskulatur, die per se zu einem klinisch oft ausgedehnten Hernienbefund führt, dem Grundsatz der Narbenhernienchirurgie „Augmentation statt Bridging“ nicht gerecht werden. Es resultieren unbefriedigend hohe Rezidivraten, im Falle einer Wundinfektion droht die Netzinfektion und damit der Implantatverlust.

Methodik. Die Komponentenseparation nach Ramirez ist ein bewährtes chirurgisches Verfahren zur Transposition der Rektusmuskulatur nahe in die Medianlinie, somit kann der netzverstärkte Sublay-Repair als optimales Verfahren zur Herniensanierung in klassischer Weise häufig durchgeführt werden. Dieser Eingriff hat auf Grund der Größe des Präparationsdefektes eine nicht unwesentlich hohe Morbidität (Haematome, Serome, Wundinfekte). Ein verbessertes Verfahren mit wesentlich geringerer Wundfläche stellt die minimal invasive Komponentenseparation nach Rosen dar.

Ergebnisse. Nach Durchführung von 4 MIC-Komponentenseparationen bei ausgedehnten Narbenhernie lässt sich das Verfahren als praktikabel und effizient hinsichtlich Mobilisation der Rektusmuskulatur beschreiben. In unserer geringen Fallzahl zeigte sich keine eingriffsbedingte Morbidität, insbesondere keine Wundkomplikationen. Der Schmerzmittelverbrauch war deutlich geringer als bei der Ramirez-Technik.

Schlussfolgerungen. Die MIC-Komponentenseparation stellt unserer Meinung nach eine bedeutende Verbesserung der chirurgischen Möglichkeiten zur Sanierung ausgedehnten Narbenhernien der Mittellinie dar. Die
Indikation ist sicherlich Ausnahmesituationen von großen Narbenhernien vorbehalten. Weitere klinisch Studien sind erforderlich.

\section{7}

\section{Erfahrungen in der operativen Therapie von Parastomalhernien anhand von 33 Fällen in 10 Jahren}

\section{G. Köhler, M. Aufschnaiter}

BHS Linz, Chirurgie, Linz, Austria

Die Inzidenz von Parastomalhernien bei endständigen stomata liegt bei circa $50 \%$. Davon werden ca $10-15 \%$ operativ versorgt. Die Indikation dafür besteht bei Entleerungsstörungen, schlechter Versorgbarkeit, peristomalen Hautschäden und auch aufgrund des kosmetischen Anspruches mancher Patienten.

Die direkte Fasciennaht weist Rezidivraten von bis zu $70 \%$ auf und ist heute nicht mehr zu befürworten. Auch Stomaneuanlagen mit Rezidivraten von bis $\mathrm{zu} 40 \%$ und Schwächung eines zweiten Quadranten der Bauchdecke sind nicht empfehlenswert.

An offenen Netzverfahren stehen die Onlay und Sublaytechniken mit Wundinfektraten von ca $7 \%$ und Rezidivraten von ca $10-20 \%$, weil die Schwachstelle innen beim Durchtritt des Darmes durch das Peritoneum nicht versorgt wird, zur Verfügung.

Besser geeignet scheinen die open IPOM und lap IPOM Techniken zu sein.

An unserer Abteilung wurden von 01.01.2000 bis 31.12.2010 33 Patienten mit Parastomalhernien (Paracolostomie, Ileostomie und Urostomie) in unterschiedlichen Techniken operiert.

Es traten 5 perioperative Komplikationen in Form von drei Wundinfekten, einer Stomastenose und einer Stomanekrose auf.

Es gab zwei Hernienrezidive jeweils nach direktem Fascienverschluss im Rahmen einer Rückverlagerung einer doppelläufigen Schutztransversostomie.

\section{8}

Chirurgische Versorgung großer Narbenhernien bei organtransplantierten Patienten mit dermalem (Schweine) Kollagen $\left(\right.$ Permacol $\left.^{\mathbb{B}}\right)$

\section{G. Werkgartner ${ }^{1}$, D. Kniepeiss ${ }^{2}$, D. Wagner ${ }^{2}$, H. Mischinger ${ }^{1}$}

${ }^{1}$ Klin. Abt. f Allgemeinchirurgie, Graz, Austria;

${ }^{2} \mathrm{Klin}$. Abt. f Transplantationschirurgie, Graz, Austria 
Grundlagen. Die Inzidenz für große Narbenhernien nach medianer Laparotomie beträgt bis zu $20 \%$. Die Rezidivrate nach Hernienverschluß ohne Mesh liegt zwischen $30 \%$ und $50 \%$. Bei den Komplikationen rangieren Wundheilungsstörungen bzw. Netzinfektionen zwischen $20 \%$ und $46 \%$. Besonders gefährdet sind Patienten mit großen Narbenhernien nach Organtransplantationen. Seit wenigen Jahren steht neben synthetischen Herniennetzen auch dermales (Schweine)Kollagen für die Bauchdeckenkonstruktion zur Verfügung. Wir untersuchten restrospektiv die Eignung von Permacol ${ }^{\circledR}$ für den Verschluß großer Narbenhernien.

Methodik. Im Jahre 2010 wurden 14 Patienten (10 Männer; 4 Frauen) wegen großer Narbenhernien nach Organtransplantationen chirurgisch versorgt. Das Mittlere Alter lag bei $60 \pm 5$ Jahren, die mittlere Aufenthaltsdauer bei $8 \pm 3,2$ Tagen. Permacol ${ }^{\circledR}$ wurde in allen Fällen in Sublay-Technik eingenäht. Beurteilt wurde der unmittelbare postoperative Outcome, die Patientenzufriedenheit sowie die 30 Tage Morbidität.

Ergebnisse. Permacol wurde in allen Fällen trotz aufrechter Immunsuppression gut vertragen. Schwerwiegende postoperative Komplikationen oder gar Reherniationen konnten nicht beobachtet werden, lediglich in 2 Fällen kam es zum Auftreten von größeren Hämatomen. Die Mobilisierung der Patienten erfolgte ab dem 1. postoperativen Tag. Die Patientenzufriedenheit war wegen des geringen „Fremdkörpergefühls“ ausgesprochen hoch.

Schlussfolgerungen. Die Verwendung von Permacol zum Verschluß großer Narbenhernien organtransplantierter Patienten ist ein sicheres Verfahren mit hoher Patientenzufriedenheit und guten kosmetischen Ergebnissen.

\section{Chirurgische Forschung III: Onkologie, Transplantation}

\section{9}

\section{Comparison of lymphangiogenesis between primary colorectal cancer and corresponding liver metastases}

\section{S. F. Schoppmann ${ }^{1}$, A. Schoppmann ${ }^{1}$, D. Tamandl', F. Längle ${ }^{2}, T$. Grünberger ${ }^{3}$ \\ ${ }^{1}$ Universitätstsklinik für Chirurgie, Vienna, Austria; ${ }^{2}$ Wiener Neustadt, Wiener Neustadt, Austria; ${ }^{3}$ Universitätstsklinik für Chirurgie, Wien, Austria}

Background. Liver metastasis (LM) is one of the determining factors of a poor prognosis in colorectal cancer (CRC). The lymphatic system is the primary pathway for metastatic spread in CRC and tumor-asso- ciated lymphangiogenesis plays a major role in the process of metastasis in various tumor entities. Peripheral lymphatico-venous communications have been discussed as a potential pathway of tumor cells for the development of LMs.

Methods. Here we investigate the clinical impact of the lymphangiogenic tumor-activity in CRCs and their corresponding LMs. From 47 patients with CRC, primary tumors and the corresponding LMs were investigated. Lymphangiogenesis (LMVD), lympho-vascular invasion (LVI) and the expression of VEGF-C were measured. A significant correlation was seen between LMVD and LVI ( $p=0.001 / p=0.0001$, respectively). LMVD-CRC correlated significantly with LMVD-LM $(p=0.026)$ and LVILM $(p=0.036)$. A significant association was found between CRC-LVI and the lymph-node status $(p=0.04)$. Univariate survival analysis showed a significant difference in DFS and OS between patients with and without VEGF-C-expression in LM $(p=0.0019$ and $p=0.0101$, respectively).

Conclusions. In summary these data provide evidence for an important role of lymphangiogenesis in colorectal cancer liver-metastasis and provide further support for a possible role of a lymphatico-venous metastatic pathway with possible high therapeutic implications.

\section{0}

\section{Genexpression in fetalem und adultem Pankreasgewebe}

\section{P. Hengster ${ }^{1}$, E. Presul ${ }^{2}$, M. Maglione ${ }^{1}$, C. Sergi ${ }^{1}$, M. Hermann ${ }^{2}$}

${ }^{1} \mathrm{MUI}$, Innsbruck, Austria; ${ }^{2} \mathrm{KMT}$ Labor MUI, Innsbruck, Austria

Grundlagen. Wachstumsfaktoren steuern die Zellteilung und Zelldifferenzierung. In der fetalen oder auch frühen postpartalen Periode sind Zellvermehrungen natürlich, während im Erwachsenalter Regeneration von Gewebe eingeschränkt möglich ist. Insbesondere bei Diabetes werden einmal zerstörte Betazellen nie mehr im erforderlichen Ausmaß nachgebildet. Ziel dieser Untersuchung war es die Genexpression zu vergleichen um Rahmenbedingungen für die Vermehrung und Differenzierung von Zellen im Labor zu definieren.

Methodik. Nach Genehmigung durch die Ethikkommission und Einwilligung der Betroffenen wurden fetales und adultes Pankreas-, Milz- und Lebergewebe zur Untersuchung eingefroren. Nach RNA Extraktion wurde mittels CHIP Analyse die Expression der Gene untersucht.

Ergebnisse. Es konnten über 100 Gene gefunden werden die ausschließlich in fetalem Gewebe exprimiert wurden. Diese Gene sind unterschiedlichen Signalübertragungswegen zuzuordnen.

Schlussfolgerungen. Die Definition fetaler Genexpression ist die Basis für die Etablierung einer Umgebung von Wachstums- und Regeulationsfaktoren für die in vitro 
Expansion von insulinproduzierenden Zellen und die erfolgreiche Zelltherapie von Diabetes.

\section{1}

The role of neuronal nictric oxide synthase in ischemia reperfusion injury in a murine pancreas transplantation model

\section{B. Cardini ${ }^{1}$, G. Brandacher ${ }^{2}$, M. Hermann ${ }^{3}$, K. Watschinger ${ }^{1}$, R. Oberhuber ${ }^{2}$, P. Obrist ${ }^{4}$, R. Margreiter ${ }^{2}$, J. Pratschke ${ }^{2}$, E. R. Werner ${ }^{1}$, M. Maglione ${ }^{2}$}

${ }^{1}$ Division of Biological Chemistry, Biocentre, Innsbruck Medical University, Innsbruck, Austria; ${ }^{2}$ Center of Operative Medicine, Department of Visceral, Transplant and Thoracic Surgery, Innsbruck Medical University, Innsbruck, Austria; ${ }^{3}$ Department of Anesthesiology and Critical Care Medicine, Innsbruck Medical University, Innsbruck, Austria; ${ }^{4}$ Institute of Pathology, St. Vinzenz Hospital, Zams, Austria

Background. Tetrahydrobiopterin (H4B) donor pretreatment significantly attenuates ischemia reperfusion injury (IRI) related pancreatitis in a murine pancreas transplantation (PTX) model. To gain further mechanistic insights, we aimed to investigate, if the neuronal nitric oxide synthase is the major target of H4B-mediated protection using nNOS knock-out (-/-) mice.

Methods. In a heterotopic PTX-model syngeneic C57BL6 mice (wild-type and nNOS-/-) were used as donor-recipient pairs. Grafts were subjected to $16 \mathrm{~h}$ cold ischemia time (CIT). Donors were either pre-treated with $50 \mathrm{mg} / \mathrm{kg} \mathrm{H} 4 \mathrm{~B}$ or untreated. Non-transplanted animals served as controls. Following $4 \mathrm{~h}$ of reperfusion, graft microcirculation was analyzed by intravital fluorescence microscopy. Parenchymal damage and peroxynitrite-formation were histopathologically and immunohistochemically assessed, respectively. H4B levels were determined by HPLC.

Results. While prolonged CIT significantly reduced microcirculation in wild-type grafts, no damage was observed in untreated nNOS-/- grafts. H4B-pre-treatment significantly restored capillary blood flow in wild-types, however, no further beneficial effect was observed in nNOS-/- organs. Furthermore, in nNOS-/- grafts neither parenchymal damage nor nitrotyrosine formation was significantly influenced by H4B treatment. Intragraft H4B levels were not affected in nNOS-/- grafts by H4B pre-treatment.

Conclusions. These results observed in mice lacking nNOS suggest a crucial involvement of this enzyme in promoting IRI in this model.

\section{2}

\section{Donor HSCs from the bone component of CTA migrate to recipient thymus and differentiate to mature $\mathrm{T}$ cells}

\section{R. Sucher ${ }^{1,2}$, C. Lin $^{2}$, B. Kern², W. Lee ${ }^{2}$, J. Pratschke ${ }^{1}$, S. Schneeberger, ${ }^{1,2}$, G. Brandacher ${ }^{1,2}, \mathrm{X}$. Zheng ${ }^{3}$}

${ }^{1}$ Department of Visceral- Transplant- and Thoracic Surgery, Innsbruck, Austria; ${ }^{2}$ Department of Plastic and Reconstructive Surgery, Johns Hopkins University School of Medicine, Baltimore, USA;

${ }^{3}$ Department of Plastic and Reconstructive Surgery, UPMC, Pittsburgh, USA

Background. Composite-Tissue-Allotransplantation (CTA) is immunologically unique in that it represents the only type of graft to include a vascularized functional bone-marrow-component. Here we studied if the bonecomponent of a composite-tissue-graft represents the source of HSCs that differentiate in the thymus and thereby reconstitute a functional immune system (CD3+ $\mathrm{T}$ cells/in-peripheral-blood/lymphoid-organs) in immunodeficient B6/SCID-recipients.

Methods. B6 (WT/nude) murine-composite-tissuegrafts (osteomyocutaneous/myocutaneous) were transplanted heterotopically to $\mathrm{B} 6$ (WT/scid) recipients. Flow-cytometry of peripheral-blood (CD3, CD19) was performed at pod: $7,14,21,28,56$. Histopathology (H\&E) and immunohistochemistry of tissues was performed. To assess immunocompetence, allogeneic skin grafts $(\mathrm{Balb} / \mathrm{c})$ were transplanted to either naïve B6/nude, naïve $\mathrm{B} 6 / \mathrm{scid}$ or $\mathrm{B} 6 /$ scid mice that prior received a B6/nude CTA.

Results. The surgical-success-rate was $85 \%$. No CD3+ cells and no rejection of skin-allografts were detected in $\mathrm{B} 6 /$ nude and $\mathrm{B} 6 /$ scid controls. B6/scid mice that received B6/nude osteomyocutaneous flaps demonstrated B-and T-cell immunity from pod 7-and 21-respectively. The percentage of $\mathrm{CD} 3+$ and CD19+ cells within peripheralblood-mononuclear-cells steadily increased to $57.7 \%$ and $17.1 \%$ respectively. Allogeneic-skin-allografts were rejected 2 weeks after transplantation. However, no $\mathrm{B}$-and T-cell-reconstitution was observed in B6/scid mice receiving $\mathrm{B} 6 /$ nude myocutaneous flaps (without bone component).

Conclusions. The vascularized-bone-marrow-component of CTA provides an effective source of HSCs to restore immunocompetence in T-and B-cell deficient mice. This might also contribute to chimerism-induction and maintenance after CTA and facilitate the clinically observed immunoprivilege of CTAs. 


\section{3}

\section{Human thyroid tumor xenografts in the athymic nude rats}

D. E. Malliga ${ }^{1}$, G. Wolf ${ }^{2}$, M. Y. Rigler ${ }^{2}$, A. Kresse ${ }^{3}$, M. Rossmann-Tsybrovskyy ${ }^{4}$, E. C. Prandl ${ }^{5}$, S. Uranüs ${ }^{1,6}, 0$. Tsybrovskyy ${ }^{4}$

${ }^{1}$ Medizinische Universität Graz/Universitätsklinik für Chirurgie/ Klinische Abteilung für Herzchirurgie, Graz, Austria;

${ }^{2}$ Medizinische Universität Graz/Universitätsklinik für Chirurgie/ Klinische Abteilung für Algermeinchirurgie, Graz, Austria; ${ }^{3}$ Medizinische Universität Graz/Intitut für Pathophysiologie und Immunologie, Graz, Austria; ${ }^{4}$ Medizinische Universität Graz/Institut für Pathologie, Graz, Austria; ${ }^{5}$ Medizinische Universität Graz/Universitätsklinik für Chirurgie/Klinische Abteilung für Plastische und Rekonstruktive Chirurgie, Graz, Austria; ${ }^{6}$ Medizinische Universität Graz/Universitätsklinik für Chirurgie/Klinische Abteilung für Chirurgische Forschung, Graz, Austria

Background. Development of an in vivo thyroid tumor model using surgical implantation of histologically intact tumor specimens.

Methods. Fresh $1-2 \mathrm{~mm}^{3}$ intraoperative specimens from 7 papillary carcinomas, 2 follicular adenomas, 1 medullary carcinoma and 4 normal thyroids were implanted subcutaneously in three athymic naked mice (Hsd:RHFoxnlrnu) respectively. Some rats were treated by weekly intraperitoneal injections of chlodronate liposomes (to deplete macrophages) and anti-asialo-GM1-antibodies (to deplete NK cells). The implants were inspected weekly and removed at different time points for histological study.

Results. All but two xenografts $(93 \%$, including both normal thyroid and all tumor types) were rejected within 4 weeks. The rejection was mediated by macrophages (first/second week) and later by NK cells (second/third week). In all cases, rejection could be completely prevented by macrophage and NK-cell depletion, as indicated both by continuous xenograft growth and no signs of rejection upon histologic examination. After discontinuation of the treatment, all but one rat (94\%) rejected their xenografts by the same mechanisms as untreated rats.

Conclusions. Rejection mediated by macrophages and NK cells precludes the growth of human thyroid xenografts in the naked rat. Continuous depletion of macrophages and NK cells prevents rejection and allows continuous xenograft growth in nearly all cases.

\section{4}

Nutrazeutika verbessern die Organfunktion in einem Rattennieren-IschämieReperfusionsmodell

\author{
P. Gehwolf ${ }^{1}$, F. M. Struller ${ }^{1}$, A. Kostron ${ }^{1}$, M. Wolzt ${ }^{2}$, \\ F. H. Bach ${ }^{3}$, L. E. Otterbein ${ }^{3}$, B. Wegiel ${ }^{4}$, J. Pratschke ${ }^{1}$, \\ R. Ollinger ${ }^{1}$
}

${ }^{1}$ Univ.-Klinik Innsbruck, Visceral-, Transplantationsund Thoraxchirurgie, Innsbruck, Austria; ${ }^{2}$ Medizinische Universität Wien, Universitätsklinik für klinische Pharmakologie, Wien, Austria; ${ }^{3}$ Department of Surgery, Transplant Center, Beth Israel Deaconess Medical Center, Harvard Medical School, Boston, USA; ${ }^{4}$ Department of Surgery, Transplant Center, Beth Israel Deaconess Medical Center, Harvard Medical School, Bosoton, USA

Grundlagen. Die zum Hämabbau notwendige induzierbare Hämoxygenase-1 (HO-1) wirkt stark antiinflammatorisch und schützt vor Ischämie- und Reperfusionsschäden (IRS). Eine klinische Anwendung im Sinne einer HO-1-Induktion ist aufgrund der Hepatotoxizität der experimentell verwendeten HO-1-Induktoren nicht möglich. Ziel der Studie war es, natürlich vorkommende Nutrazeutika auf Ihr Potential zur HO-1 Induktion zu untersuchen.

Methodik. Verschiedene Nutrazeutika wurden in Hinblick auf die HO-1-Induktion mittels PCR getestet. In einem etablierten Ratten-Nierenarterien-Klemmmodell wurde ein IRS gesetzt, Nierenfunktionsparameter und HO-1 Expression wurden zu festgelegten Zeitpunkten bestimmt, histologische Untersuchungen wurde durchgeführt. Die Nutrazeutika wurden $24 \mathrm{~h}$ vor Ischämie und unmittelbar nach Reperfusion oral appliziert.

Ergebnisse. Zwei der getesteten Nutrazeutika führten $\mathrm{zu}$ einer starken Hochregulation (Resveratrol: 11-fach, Ginseng: 17-fach) der HO-1 Expression. Im Nierenarterien-Klemmmodell kam es nach 48 Stunden zu einem Anstieg des Serum Kreatinin von $0,38 \mathrm{mg} / \mathrm{dl} \pm 0,07$ auf $3,06 \mathrm{mg} / \mathrm{dl} \pm 0,86$ in den Kontrolltieren. Die Applikation von beiden Nutrazeutika in einer Dosierung von $10 \mathrm{mg} /$ $\mathrm{kg}$ (Resveratrol, 48h-Kreatinin $0,54 \mathrm{mg} / \mathrm{dl} \pm 0,23$ ) bzw. $30 \mathrm{mg} / \mathrm{kg}$ (Ginseng, $48 \mathrm{~h}$-Kreatinin $0,53 \mathrm{mg} / \mathrm{dl} \pm 0,06$ ) verhinderte dramatisch die Einschränkung der Nierenfunktion ( $p<0,0001$ für beide $v s$. Kontrolle). Die kompetitive Antagonisierung durch SnPP (5 mg/kg/KG) konnte diesen positiven Effekt vermindern. Histologische Auswertungen unterstützen die Ergebnisse.

Schlussfolgerungen. Die Anwendung von für Menschen ungefährlichen Nutrazeutika stellt eine ausgezeichnete Möglichkeit dar, die HO-1 zu induzieren und den IRS zu minimieren.

\section{5}

\section{Effects of bile pigments on renal ischemia reperfusion injury and novel strategies for its application}

\author{
T. Resch ${ }^{1}$, C. Koidll, F. Bösch ${ }^{1}$, M. Thomas ${ }^{1}$, \\ C. Eisenbach ${ }^{1}$, P. Gehwolf ${ }^{1}$, A. Weissenbacher ${ }^{1}$, \\ K. Stromberger ${ }^{1}$, R. Sucher ${ }^{1}$, P. Kogler ${ }^{1}$, F. Bach ${ }^{2}$, \\ J. Pratschke ${ }^{1}$, R. Öllinger ${ }^{1}$ \\ ${ }^{1}$ Universitätsklinik für Visceral-, Transplantations- und \\ Thoraxchirurgie Innsbruck, Innsbruck, Austria; \\ ${ }^{2}$ Immunobiology Research Center, Harvard Medical \\ School/BIDMC, Boston, USA
}


Background. Ischemia-reperfusion injury (IRI) represents an unresolved issue in renal transplantation. Recent findings indicate that the bile pigment bilirubin can exert beneficial effects.

Methods. In two Wistar rat models of renal IRI, kidneys were either transplanted to isogenic recipients $(18 \mathrm{~h}$ cold ischemia), or, in a second model, renal artery clamping of contralaterally nephrectomised rats was performed (45 min). Various doses $(1-30 \mathrm{mg} / \mathrm{kg})$ of bilirubin, or its precursor biliverdin, were administered i.v. pre-, peri-, or postoperatively. Serum bilirubin was quantified shortly after reperfusion. Serum urea and creatinine were analyzed after 24 and $48 \mathrm{~h}$

Results. Pre- and perioperative administration of bilirubin/biliverdin ditaurate demonstrated dose dependent bioavailability (ED 3-30 mg/kg). Creatinine and urea levels declined significantly already after $24 \mathrm{~h}$ in the clamp model. Dose elevation over $10 \mathrm{mg} / \mathrm{kg}$ did not prove to be beneficial. Interestingly, postoperative administration showed to be ineffective. Analogous findings were observed in the transplantation model, although higher dosages of biliverdin were required $(>30 \mathrm{mg} / \mathrm{kg}$ ).

Conclusions. I.v. application of bile pigments is effective in preventing renal transplant malfunction from IRI. Bilirubin serum levels must be increased prior to reperfusion. The application of bile pigments in renal transplantation might serve as a novel strategy in the prevention of IRI.

\section{6}

Inhibition of p38 kinase prevents functional impairment resulting from kidney clamping

\section{Wallner ${ }^{1}$, M. Ashraf ${ }^{1}$, M. Ebner ${ }^{1}$, S. Vallant ${ }^{1}$,}

\section{A. Soleiman ${ }^{2}$, J. Troppmair ${ }^{1}$}

${ }^{1}$ Department of Visceral-, Transplant- and Thoracic Surgery, Medical University of Innsbruck, Innsbruck, Austria;

${ }^{2}$ Soleiman Pathology, Hall, Austria

Ischemia/reperfusion injury (IRI) during transplantation leads to organ malfunction and constitutes a major unsolved problem in transplantation medicine. In particular transition from ischemia to reperfusion is marked by an increase in intracellular signaling activity and a deterioration of mitochondrial function marked by excessive ROS production and $\mathrm{Ca}^{2+}$ overload. Our work had identified the mitogen activated protein kinase (MAPK) p38 as a potential negative regulator of these mitochondrial alterations which are crucial for the development of IRI. We thus wanted to test whether p38 inhibition also limited IRI in an in vivo model. In our studies the reperfusion following kidney clamping in the Lewis rat showed pronounced activation of $\mathrm{p} 38$ following as observed in similar settings before. The use of p38 kinase inhibitor BIRB 796 efficiently blocked p38 activation at a dose of $5 \mathrm{mg} / \mathrm{kg}$ as well as that of the downstream target MK2 while the activation of the kinases ERK1,2 and JNK1,2 was unaffect- ed. Most importantly, kidney function as monitored by the levels of serum creatinine and urea was strikingly better preserved in animals receiving the inhibitor. Taken together these data support the beneficial effect of p38 inhibition in the prevention of IRI.

\section{Österreichische Gesellschaft \\ für Thorax- und Herzchirurgie: Herzchirurgie - TAVI und Neu Aortenklappenprothesen}

\section{0}

\section{Jahresergebnisse TA-TAVI in Linz}

\section{J. Demmer, M. Alavian, C. Groß}

Chirurgie 1, Linz, Austria

Zwischen XII/2008 und XII/2010 wurden 37 TA-TAVIOperationen durchgeführt (24 weiblich, 13 männlich, Altersschnitt 81,7 Jahre, mittlerer log. Euroscore 34,6). 28 Patienten hatten keine Herzoperation in der Anamnese, 9 Patienten waren voroperiert; der mittlere präop. transvalvuläre Gradient betrug $62,4 \mathrm{mmHg}$; bei 14 Patienten wurde eine $23 \mathrm{~mm}$ Edwards Sapienklappe, bei 23 eine $26 \mathrm{~mm}$ Klappe implantiert; der mittlere postop. transvalvuläre Gradient betug 14,7 mmHg; in 26 Fällen fand sich kein paravalvuläres, in 11 Fällen ein triviales im postop. TEE; bei 1 Patientin musste wegen subtotaler Okklusion des linken Ostiums auf eine Sternotomie umgestiegen werden, 1 anderer Patient wurde wegen diffuser Blutung im Bereich der Herzspitze über die Femoralgefäße an die HLM genommen; beide Patienten überlebten ohne kardiale bzw. neurologische Folgeerscheinungen. Die 30 Tage Mortalität betrug 5,4\% $(n=2)$.

Schlussfolgerungen. TA-TAVI-Operationen können bei Hochrisikopatienten mit akzeptabler Morbidität und Mortalität durchgeführt werden.

\section{1}

\section{Intraoperative and intermediate-term results of an interdisciplinary Transcatheter Aortic Valve Implantation (TAVI)-program}

\author{
A. Heinz ${ }^{1}$, N. Bonaros ${ }^{1}$, T. Bartel ${ }^{2}$, S. Müller ${ }^{2}$, \\ G. Friedrich ${ }^{2}$, O. Pachinger ${ }^{2}$, M. Grimm¹ ${ }^{1}$, L. Müller ${ }^{1}$ \\ ${ }^{1}$ Department of Cardiac Surgery, Medical University Innsbruck, \\ Innsbruck, Austria; ${ }^{2}$ Department of Cardiology, Medical \\ University Innsbruck, Innsbruck, Austria
}


Background. The aim of the study was to evaluate results of the first three years of our institutional TAVIprogram in high risk patients, who were excluded from conventional surgical aortic valve replacement due to increased risk

Methods. From February 2008 till December 2010, 52 cases with severe aortic stenosis were included in our TAVI-program by a cardiac surgical -cardiological team. The access was transapical in 26 patients, transfemoral in 18 and transaxillary in 3 . The median age was 82 years (range 58-94) and median EURO Score 29.6\% (range $6.2-74.5 \%) .14$ of 52 cases have had previous cardiac surgery. The median follow up period was 304 days (1-1058 days).

Results. Forty-eight patients had successful TAVI, 2 needed to be converted to surgical replacement, 2 had balloon valvuloplasty only and 10 patients needed PCI before TAVI. The perioperative mortality (30 days) was $5.88 \%$ (3 patients) and during follow up $13.73 \%$ (7 patients). No patient suffered myocardial infarction, 2 had peri-operative stroke and 2 during follow up. In 9 patients peri-operative renal failure needing hemofiltrations was observed.

Conclusions. Due to interdisciplinary cooperation of cardiac-surgery and cardiology our TAVI program implying transfemoral, transapical and transaxillary access could be established with low complication- and mortality-rate.

\section{2}

Transapical valve implantation in high risk patients - initial experience at the Herzzentrum Hietzing - Vienna

\section{F. H. Veit ${ }^{1}$, S. Folkmann ${ }^{1}$, H. Pollak ${ }^{2}$, M. Thalmann', M. Grabenwöger ${ }^{1}$}

${ }^{1} 1$ st Surgical Department Vienna-Hietzing, Vienna, Austria; ${ }^{2} 4$ th Medical Department of Vienna -Hietzing, Vienna, Austria

Background. Transapical aortic valve implantation (TAVI) is an alternative treatment option for patients facing high risk aortic valve implantation avoiding CBP and sternotomy.

Methods. Twelve consecutive high risk pts with severe symptomatic aortic stenosis received TAVI using Edwards Sapien $^{\mathrm{TM}}$ pericardial Xenograft between $4 / 2010$ and $11 / 2010$. Mean pts age was 80,7 yrs, f:m $=9: 3$, with a mean log EuroScore of $30.4 \%$ and mean EF of $52.5 \%$. In $3(25 \%)$ a CABG procedure was performed previously.

Results. Device delivery was successful in $11 / 12$ $(91.6 \%)$ pts. Thirty day mortality was $8.3 \%(1 / 12)$ due to bleeding, 1 pat $(8.3 \%)$ had to be converted into open AVR (misdiagnosed bicuspid AV) because of ventricular migration. The incidence of stroke or permanent PM implantation was $0 / 12$. Postoperative echocardiography revealed no PVL in $8 / 11$ (72.7\%), $1+$ AR in $3 / 11$ (27.3\%). No pat died during follow up period.
Conclusions. Off pump minimal invasive TAVI is a reasonable alternative to open AVR in high risk patients with severe symptomatic aortic stenosis and should be considered in elderly and previously operated patients.

\section{3}

\section{Hybrid treatment of aortic stenosis and coronary artery disease by transcatheter aortic valve replacement and percutaneous coronary intervention}

A. Heinz ${ }^{1}$, L. Müller ${ }^{1}$, T. Bartel ${ }^{2}$, S. Müller ${ }^{2}$, G. Friedrich², O. Pachinger ${ }^{2}$, M. Grimm ${ }^{1}$, N. Bonaros ${ }^{1}$

${ }^{1}$ Department of Cardiac Surgery, Medical University Innsbruck, Innsbruck, Austria; ${ }^{2}$ Department of Cardiology, Medical University Innsbruck, Innsbruck, Austria

Background. The presence of coronary artery disease (CAD) is a significant risk factor for increased mortality in patients undergoing aortic valve replacement for severe aortic stenosis (AS). Transcatheter aortic valve replacement shows promising.

Results. The aim of the study was to analyse the short and mid-term outcome of hybrid treatment of patients with AS and CAD by percutaneous coronary intervention (PCI) and transcatheter aortic valve implantation (TAVI).

Methods. From February 2008 till December 2010, 10 patients with severe AS and CAD were treated with TAVI and PCI; the male:female ratio was 8:2. The median age was 82.5 years (range 79-88) and median logistic EURO Score $28.4 \%$ (range $8.1-74.5 \%$ ). The follow up period was 238 days (range $43-564$ days).

Results. All 10 patients received successfully a biological aortic valve implatation. Twelve bare metal coronary stents were implanted ( 2 patients received 2 stents). Median interval between PCI and TAVI was 28.5 days (range 2-210 days). We observed no peri-operative mortality (30days). Three patients died during follow up (between 43 and 84 days post-operative). No coronary or valvular reintervention was required.

Conclusions. Combined treatment of concomitant $\mathrm{CAD}$ and AS by transcatheter procedures for high risk patients provides satisfactory peri-operative and 1-year results.

\section{4}

Erste hämodynamische Ergebnisse nach Aortenklappenersatz mit der neuen TRIFECTA ${ }^{\text {TM }}$ Perikard Bioprothese

\section{R. Moidl, S. Folkmann, G. Weiss, K. Kornigg, M. Gorlitzer, M. Grabenwöger}

I. Chirurgie, KH Hietzing, Vienna, Austria 
Grundlagen. Die hämodynamisch optimierte Konstruktion der Trifecta ${ }^{\mathrm{TM}}$ Bioprothese führt $\mathrm{zu}$ einer vollständigen Öffnung der drei Klappentaschen in Aortenposition wie bei einer natürlichen Herzklappe. Die Zielsetzung unserer Studie war es, erste echokardiographische Untersuchungen an 20 Patienten nach Aortenklappenersatz (mittleres Alter 76,2 $\pm 7,5$ Jahre) zum Zeitpunkt der Entlassung zu evaluieren.

Methodik. Alle Patienten erhielten nach Dekalzifizierung der erkrankten Aortenklappe einen supra-anulären Aortenklappenersatz mit pledgetarmierten Klappennähten, $67 \%$ zusätzlich aortokoronare Bypässe. Bei Entlassung wurde eine transthorakale Echokardiographie mit Evaluierung der Prothesenfunktion durchgeführt: Flußgeschwindigkeiten $\left(\mathrm{AV}_{\max }+\right.$ mean), Gradienten $\left(\mathrm{P}_{\max }+\right.$ mean), als auch effektive und indizierte Öffnungsflächen (EOA, EOAI) wurden berechnet. Signifikanter PatientProsthesis-Mismatch (PPM) wurde definiert bei $\mathrm{EOAI}<0,6 \mathrm{~cm}^{2} / \mathrm{m}^{2}$.

Ergebnisse. Abhängig von der Klappengröße (19-27) waren die Flußgeschwindigkeiten (m/sec): $A_{\text {max }}: 2,7 \pm$ 0,4 bis $1,8 \pm 0,3$; $\mathrm{AV}_{\text {mean }} 2,1 \pm 0,3$ bis $1,4+/ 0,2$; die Gradienten (mmHg): $\mathrm{P}_{\text {max }}$ : $29,7 \pm 9,3$ bis $13,6 \pm 2,8$; $\mathrm{P}_{\text {mean: }}$ : $19,2 \pm 3,7$ bis 7,6 $\pm 1,6$. Diese optimierten echokardiographischen Parameter führten in keinem der 20 Patienten, auch in kleinen Größen (19/21 $n=10)$ zu einem signifikanten PPM bei der Entlassung.

Schlussfolgerungen. Die Konstruktion der neuen Trifecta $^{\mathrm{TM}}$ Bioprothese erlaubt eine optimale Hämodynamik nach Aortenklappenersatz gerade in Patienten mit kleinen Aortenwurzeln. Langzeituntersuchungen müssen zeigen, ob dadurch eine verbesserte Lebensqualität als auch Überleben der Patienten erreicht werden kann.

\section{5}

\section{Minimalinvasiver Aortenklappenersatz mit nahtloser Bioprothese}

\section{T. Fischlein, G. Santarpino, S. Pfeiffer}

Klinikum Nürnberg - Klinik für Herzchirurgie, Nürnberg, Germany

Grundlagen. MIS wird für multimorbide Patienten zum Aortenklappenersatz vorgeschlagen. Wir präsentieren unsere frühen Ergebnisse nach minimalinvasiver nahtloser Implantation einer Aortenklappenprothese.

Methodik. Die „Sorin Perceval“ ist eine von einem Nitinolstent gestützte Rinderperikardprothese, welche nahtlos in den Aortenanulus offen chirurgisch implantiert wird. Im Rahmen einer Zertifizierungsstudie wurden 39 Patienten (Pat) untersucht. Alle isolierten Aortenklappenersatzpatienten wurden durch MIS (partielle Sternotomie, Klappenersatz mit Herz-Lungen Maschine) versorgt.

Ergebnisse. 15 Pat erfüllten die Einschlusskriterien nicht (Bikuspide AK, Anulus $>25 \mathrm{~mm}$ ). 24 Pat erhielten eine 21 (3), 23 (9) oder $25 \mathrm{~mm}$ Prothese (12), isoliert $(n=19)$ oder als Kombinationseingriff $(n=5)$. LogEuroScore war $9.1 \pm 5.2$. Klemmzeiten: $47 \pm 10 \mathrm{~min}$ (isoliert) und $75 \pm 42 \mathrm{~min}$ (Kombinationseingriffe). Implantationszeit: $9.3 \pm 11.4 \mathrm{~min}$. Postoperative Echogradienten waren: $\mathrm{p}_{\max } 31.9 \pm 13.5 \mathrm{mmHg}, \mathrm{p}_{\text {mean }} 15.9 \pm 7.3 \mathrm{mmHg}$ ohne paravalvuläres Leck. 2 Pat mit transvalvuläre $\mathrm{AI} \leq 2$. 30 d-Mortalität: 0, Entlassung nach $7 \pm 0.7$ Tagen (ITS Aufenthalt $1.4 \pm 0.5$ Tage). Bei 6 Pat liegen 6-Monatsdaten vor: NYHA I bei 4 , II bei 2 Pat, $\mathrm{p}_{\max } 32.5 \pm 3.7 \mathrm{mmHg}$, pmean $16.2 \pm 1.7 \mathrm{mmHg}$, keine peri- oder transvalvuläre Insuffizienz.

Schlussfolgerungen. Die nahtfrei implantierte Perceval zeigt eine gute Hämodynamik. Für selektionierte Patienten stellt sie eine sichere Alternative bei minimalinvasiven Zugängen dar. Die Implantation ist einfach und schnell mit dem Potential, Operationszeiten zu verringern und das operative Trauma zu minimieren.

\section{6}

\section{Erste klinische Erfahrungen mit der 3f-Enable 1 Aortenklappenbioprothese}

\section{S. Folkmann, G. Weiss, M. Gorlitzer, R. Moidl, M. Grabenwöger}

Herzchirurgie, KH Hietzing, Wien, Austria

Grundlagen. Die 3f-Enable Aortenklappenbioprothese (Medtronic Inc., Minneapolis, MN, USA) representiert eine neue Generation der equinen perikardialen nahtlosen Prothese. Ziele dieser Methode sind die extrakorporale Zirkulationszeit zu minimieren, die Manipulation am Aortenannulus $\mathrm{zu}$ reduzieren und die Verbesserung der postoperativen Hämodynamik.

Methodik. Die Bioprothese in annulärer Position wurde $6(2 \mathrm{~m}, 4 \mathrm{w})$ Patienten, durchschnittlich 79 Jahre (73-86 Jahre), mit hochgradiger Aortenklappenstenose implantiert. Die Klappe ist auf Grund des selbst-expandierenden Nitinolstents nur durch eine 2,0 Naht am noncoronaren Anteil des Annulus zu implantieren. Die Komorbiditäten und die Klemmzeiten wurden evaluiert. Alle Patienten erhielten intraoperativ eine transösophageale und postoperativ vor Entlassung eine transthorakale Echokardiographie.

Ergebnisse. Die implanierten Klappengrößen waren: $23 \mathrm{~mm}: n=2,21 \mathrm{~mm}: n=2$ und $19 \mathrm{~mm}: n=2$. Einer Patientin wurde auf Grund einer Asymmetrie im Aortenannulus die Enable Prothese nicht implaniert, daher erfolgte eine Konversion auf einen konventionellen Aortenklappenersatz. Die Aortenklemmzeit betrug 42-60 Minuten (mean: 50,2 Min.). Mittels transthorakaler Echokardiographie postoperativ ergaben sich durchschnittliche transvalvuläre Gradienten von $19 \mathrm{~mm}$ : peak $32 \mathrm{mmHg}, 21 \mathrm{~mm}$ : peak $23 \mathrm{mmHg}$ und $23 \mathrm{~mm}$ : peak $19 \mathrm{mmHg}$. Der Intensivaufenthalt der Patienten betrug 
1-2 Tage, der Spitalsaufenthalt war durchschnittlich 13 Tage.

Schlussfolgerungen. Die 3f-Enable Aortenklappenprothese zeigt postoperativ ausgezeichnete hämodynamische Ergebnisse durch geringe Druckgradienten transvalvulär und eine Reduktion der Aortenklemmzeit.

\section{7}

\section{Evaluierung für LTX endete in Lebersanierung durch Peri/Epikardektomie}

\section{K. Mészáros ${ }^{1}$, B. Zirngast ${ }^{1}$, D. Wagner ${ }^{2}$, R. Stauber ${ }^{3}$, H. Mächler ${ }^{1}$, H. Müller ${ }^{2}$, F. lberer ${ }^{2}$, R. Rienmüller ${ }^{4}$, K. Tscheliessnigg ${ }^{1}$ \\ ${ }^{1}$ Klinische Abteilung für Herzchirurgie, Graz, Austria; ${ }^{2}$ Klinische Abteilung für Transplantationschirurgie, Graz, Austria; ${ }^{3}$ Klinische Abteilung für Gastroenterologie und Hepatologie, Graz, Austria; ${ }^{4}$ Univ. Klinik für Radiologie, Graz, Austria}

Grundlagen. Ein herzgesunder 65-jähriger Patient wurde zur LTX-Vorbereitung zugewiesen. Sein Leitsymptom war eine postalkoholische Leberzirrhose bei chronischer Polyserositis. Die linksventrikuläre Funktion betrug $70 \%$ ohne Hinweis auf Vitium/KHK

Methodik. Die hepatologische Untersuchung ergab eine Leberzirrhose Child-B, (MELD Score 14), die Leberbiopsie eine portale Fibrose mit läppchenzentralen Nekrosen. Echokardiographisch fand sich eine Restriktion des rechten Ventrikels, im Thorax-CT zeigte sich eine konstriktive, fibröse Peri-Epicarditis ohne Kalzifikation oder Myokardatrophie, im Cardiac MR ein EDV/ESV von $87 / 21 \mathrm{~mm}$ mit paradoxer Septum-Beweglichkeit sowie ein dip-and-plateau Phänomen (Links/Rechts-Herzkatheter). Nach Ausschluss eines Budd-Chiari-Syndroms bestand Verdacht auf eine perikarditische Pseudoleberzirrhose. Die Indikation zur Peri/Epikardektomie wurde gestellt und einschließlich des Areals um den bilateralen AV-Klappenbereich durchgeführt. Der ZVD sank von 30 auf $9 \mathrm{mmHg}$, der PAP wurde halbiert.

Ergebnisse. Neun Monate postoperativ war der Patient beschwerdefrei (NYHA-Stadium I). Im KontrollMR/CT fand sich eine ausgezeichnete Ventrikelfunktion ohne Hinweis auf Restriktion bei normalen ventrikulären Füllungsvolumina ohne Pleura- oder Perikarderguss. Das Labor ergab eine normale Leberfunktion mit Transaminasen im Normbereich, Kontrollsonographien zeigten eine normale Lebermorphologie ohne Aszites.

Schlussfolgerungen. Trotz einer primär unauffälligen kardialen Symptomatik sollte bei einer Cirrhosis hepatis eine Peri/Epikarditis mittels radiologischer Bildgebung ausgeschlossen werden. Möglicherweise konnte dem Patienten so die LTX erspart werden; die Langzeitergebnisse werden es zeigen.

\section{Hauptsitzung - Funktionserhaltung} und Lebensqualität 2

\section{8}

\section{Retroperitoneoskopische lumbale Sympathektomie bei palmoplantarer Hyperhidrosis}

\section{J. Schmidt, G. Dechantsreiter, H. Dietz \\ Krankenhaus Landshut-Achdorf, Landshut, Germany}

Grundlagen. Die CT-gesteuerte lumbale Sympathikolyse ist bei plantarer Hyperhidrosis zwar kurzfristig effektiv aber langfristig mit hoher Rezidivrate behaftet.

Methodik. In einer prospektiven Erfassungsstudie wurden 16 konsekutive Patienten mit schwerer palmoplantarer Hyperhidrosis nach erfolgreicher thorakaler Sympathektomie (ETS) zunächst CT-gesteuert behandelt. War die TestTherapie erfolgreich wurden die Patienten zweizeitig retroperitoneoskopisch sympathikolysiert. Das TEWL (total epidermal water loss) betrug präoperativ $91,5 \mathrm{~g} / \mathrm{m}^{2} / \mathrm{h}$ bzw. $94,2 \mathrm{~g} / \mathrm{m}^{2} / \mathrm{h}$. Die Retroperitoneoskopie erfolgte in Bauchlage, die Durchtrennung bei L3/L4. Die Nachkontrollen erfolgten 6 und 24 Monate nach der Therapie.

Ergebnisse. Es handelte sich um 10 weibliche und 6 männliche Patienten mit einem mittleren Alter von $31,6 \pm 8,3$ Jahren. Präoperativ bestand nach ETS bei 5 Patienten kompensatorisches Schwitzen (KS). Bei diesen verschlechterte sich das KS durch die lumbale Operation nicht. Es trat bei 4 weiteren Patienten de novo KS auf. Bei einem Mann entwickelte sich eine passagere Erektionsstörung. Eine Frau entwickelte reversible neuroradikuläre Gefühlsstörungen. Nach 24 Monaten betrug die lokale Besserung $95 \%$. TEWL weiblich lag bei $42,8 \mathrm{~g} / \mathrm{m}^{2} / \mathrm{h}$ und männlich bei $37,7 \mathrm{~g} / \mathrm{m}^{2} / \mathrm{h}$.

Schlussfolgerungen. Nach einer ETS bei palmoplantarer Hyperhidrosis kann mit einer Besserung der plantaren Symptomatik um etwa $20 \%$ gerechnet werden. Sind die Patienten weiter symptomatisch sollte die lumbale Sympathikolyse bei L4 in Erwägung gezogen werden.

\section{9}

Pulmonary thromboendarterectomy for chronic thromboembolic pulmonary hypertension in septuagenarians and octogenarians: a single center experience

\section{O. Senbaklavaci, H. Taspinar, A. Abugameh, M. Hartert, C. F. Vahl}

Klinik für Herz-Thorax-Gefäßchirurgie, Mainz, Germany 
Background. Pulmonary thromboendarterectomy (PTE) is a curative surgical procedure for chronic thromboembolic pulomonary hypertension (CTEPH). The aim of this study was to evaluate operative outcomes of septuagenarians and octogenarians undergoing PTE for CTEPH.

Methods. A retrospective analyze was performed on 179 patients who underwent PTE for CTEPH between 2002 and 2010 at our department. Twenty-nine patients (18 male, 11 female) were older than 70 years with an average age of 73,7 years (range $70-82$ years). Two patients underwent a combined PTE and aortocoronary bypass procedure.

Results. Hospital mortality was $13,8 \%$ (4/29). 3 patients died due to multi organ failure and 1 patient due to sepsis. The average length of stay on the intensive care unit was 4,5 days (range 1-11 days). Five patients had to be rethoracotomied due to postoperative bleeding. The mean length of hospital stay was 18,6 days ranging from 12 to 28 days.

Conclusions. PTE for CTEPH in septuagenarians and octogenarians can be performed with acceptable perioperative mortality and morbidity. Due to steadily increasing of population ages and life expectancy, the number of older patients referred for this procedure will be growing in the next future.

\section{0}

\section{Quality of life and clinical outcome 10 years after Nissen-fundoplication}

\section{H. F. Wykypiel ${ }^{1}$, K. Fiegl ${ }^{1}$, S. Wiplinger ${ }^{1}$, A. Perathoner', K. Glaser ${ }^{2}$, G. Wetscher ${ }^{3}$}

${ }^{1}$ Medizinische Universität Innsbruck/Universitätsklinik für Visceral-, Transplantations- und Thoraxchirurgie, Innsbruck, Austria; ${ }^{2}$ Wilhelminenspital/2. Chirurgischen Abteilung, Wien, Austria; ${ }^{3} \mathrm{KH}$ Schwaz/Chirurgische Abteilung, Schwaz, Austria

Background. In 1994, the first laparoscopic fundoplication in Austria was performed in Innsbruck. Although this operation has gained high popularity all over the world, there are only a few long-term follow up studies available ( $>10$ years). Therefore we performed a retrospective analysis of our patients ten years after Nissen fundoplication.

Methods. The first 70 patients who underwent a laparoscopic Nissen-fundoplication within a "tailored approach" where included. Clinical assessment of symptoms, gastroscopy, manometry and a 24-hours-pH-monitoring was performed prior to and six months after the operation. After a mean time of 10.5 years the patients' symptoms, their quality of life (gastrointestinal quality of life Index (GILQI), SF36) and their medical history postoperatively were assessed.

Results. Compared to preoperatively heartburn, regurgitation, hoarseness and asthma were still significantly improved ten years after Nissen-Fundoplication. Gastrointestinal Quality of life ten years after surgery was much better than with conservative treatment. After exclusion of 6 patients with otherwise severe illnesses, general quality of life was as good as in healthy people.

Conclusions. Nissen-Fundoplication is an excellent treatment option in Gastroesophageal Reflux Disease. It improves symptoms of GERD and quality of life with a long lasting effect ( $>10$ years).

\section{1}

\section{Duodenumerhaltende \\ Pankreaskopfresektion bei chronischer \\ Pankreatitis - Aktuelle Datenlage und retrospektive Analyse des eigenen \\ Patientenguts}

\section{G. Pressl, F. Kurz, M. Aufschnaiter}

BHS Linz, Linz, Austria

Die chronische Pankreatitis ist ein inhomogenes Krankheitsbild mit variablem Verlauf. Chronische Schmerzen und rez. Pankreatitisschübe stehen zumeist im Vordergrund und sind mit die häufigsten Indikationen für eine notwendige Intervention. Die endoskopischen Drainageverfahren haben die früher häufig angewandeten chirurgisch-operativen Gangdrainagen weitgehend abgelöst. Trotzdem glauben wir, dass es nach wie vor klare Indikationen für resezierende Eingriffe gibt. Wir favorisieren die „Duodenumerhaltende Pankreaskopfresektion nach Beger" bei der chron. kalzifierenden Kopfpankreatitis, wenn diese als Schrittmacher für die Erkrankung gesehen werden kann und die Linksresektion bei der schwanzbetonten chron. Pankreatitis und entsprechendem Beschwerdebild.

Im eigenen Patientengut wird die „Duodenumerhaltende Pankreaskopfresektion nach Beger" als Standard für die chron. Kopfpankreatitis seit 1996 durchgeführt. Es folgt eine retrospektive Analyse der Patientendaten bis Ende 2010 hinsichtlich Schmerzverlauf, klinisch relevanten rezidivierenden Pankreatitisschüben, endokriner und exokriner Pankreasinsuffizienz und Patientenzufriedenheit.

\section{2}

\section{Quality of live after laparoscopic} intersphincteric rectum resection

\section{P. Metzger, B. Kovanyi Holzer, K. Schmidt, S. Kriwanek, R. Schiessel}

Deparment of General Surgery, Danube Hospital, Vienna, Austria 
Background. Intersphincteric resection (ISR) of tumours with coloanal anastomosis has been implemented to avoid permanent colostomy for patients with rectal cancer located $<5 \mathrm{~cm}$ from the anal verge.

Objective. To study the anus preserving combination of transanal ISR and laparoscopic very low anterior resection of ultra-low rectal tumours.

Methods. Clinical data of 49 ultra-low rectal tumour patients without external anal sphincter involved, who underwent combined laparoscopic and ISR surgery from 2004 to 2009, were analyzed retrospectively.

Results. Cancer was classified as Stage I (8), Stage II (24), and Stage III (17) according to the TNM classification. Three patients developed neorectum necrosis and two patients anastomotic strictures. Morbidity and mortality rates were $19 \%$ and $0.0 \%$, respectively. Anal resting pressure, maximum squeeze pressure and maximum tolerance volume of the rectum decreased significantly after surgery, and restored gradually but not to normal values. Quality of life data demonstrated good results.

Conclusions. We could demonstrate that the combined approach of transanal intersphincteric and laparoscopic resection of ultra low rectal tumours respects the rules of tumour surgery, preserves the function of anus, and achieves good quality of life results.

\section{3}

Lebensqualität im Langzeitverlauf nach laparoskopischer oder konventioneller Chirugie beim Rektumkarzinom erhoben mittels EORTC QLQ-C30, QLQ-C38 und Rockwood incontinence score

\author{
R. Kafka', C. Zambanini ${ }^{1}$, B. Holzner ${ }^{2}$, J. Giesinger², \\ J. Pratschke', M. Zitt ${ }^{1}$ \\ ${ }^{1}$ Universitätsklinik für Viszeral-, Transplantations- \\ und Thoraxchirurgie, Innsbruck, Austria; ${ }^{2}$ Universitätsklinik \\ für Psychiatrie, Klin. Abt. f. Biolog. Psychiatrie, \\ Innsbruck, Austria
}

Grundlagen. Die Laparoskopie konnte im Bereich der chirurgischen Behandlung des Rektumkarzinoms Vorteile für die Rekonvaleszenz und Narbenhernienrate bei gleichwertiger onkologischer Qualität nachweisen.

Methodik. Ziel dieser retrospektiven Untersuchung ist es mittels eines standardisierten Fragenbogens der EORTC (QLQ-C30 und QLQ-C38) sowie des Rockwood incontinence scores Unterschiede in der Lebensqualität im Langzeitverlauf bei unseren Patienten mit laparoskopischer oder konventioneller Chirurgie zu erheben.

Ergebnisse. Mit der Befragung einverstanden und zum Zeitpunkt der Befragung tumorfei waren 71 Patienten: Alter 67 (36-90) mean (range); 28 (40\%) davon Frauen; 37 (53\%) mit Z.n. neoadj. RCTX; 10 (13\%) Rektumexstirpation; 18 (26\%) laparoskopisch operiert. In allen Skalen des Rockwood incontinence scores zeigt sich mit
3,4 vs. 2,8 für lifestyle, 3,4 vs. 2,9 für coping-behaviour, 3,7 $v s$. 3,5 für depression und 3,6 vs. 3,3 für embarassment ein Trend zugunsten der Laparoskopie. Mit 83 vs. 74 für physische Funktion, 79 vs. 66 für soziale Funktion, 69 vs. 57 für Rollenfunktion zeigt sich ebenfalls ein Trend zugunsten der Laparoskopie, aber kein Unterschied in anderen Bereichen wie global quality of life oder sexual function und sexual enjoyement.

Schlussfolgerungen. Obwohl die etwas zu kleine Fallzahl und die große Streuung keine signifikanten Unterschiede zulässt, scheint die laparoskopische Rektumchirurgie zumindest gleichwertige Ergebnisse in Bezug auf Lebensqualität und Inkontinenz zu bringen.

\section{Hauptsitzung - Onkologische Viszeralchirurgie}

\section{4}

The value of PET scan in the clinical $\mathrm{N}$ staging of locally advanced oesophageal cancer

C. Aigner ${ }^{1}$, J. Moons ${ }^{2}$, P. Nafteux ${ }^{2}$, W. Coosemans ${ }^{2}$, H. Decaluwe ${ }^{2}$, G. Decker ${ }^{2}$, P. De Leyn ${ }^{2}$, D. Van Raemdonck ${ }^{2}, \mathrm{~T}$. Lerut ${ }^{2}$

${ }^{1}$ Department of Thoracic Surgery, Medical University of Vienna, Vienna, Austria; ${ }^{2}$ Department of Thoracic Surgery, Leuven, Belgium

Background. Little information is available on the correlation between node-negative PET-scans and EUS/CT-scan and post-resection-findings in particular in locally advanced esophageal cancers.

Methods. Patients with pathological stage IIB or higher operated at the Department of Thoracic Surgery in Leuven between $1 / 2000$ and 12/2006 were included in the analysis. PET-node-negatives were compared to EUS/ CT and histopathological findings and related outcome.

Results. Two hundred and seventy-seven Patients were stage IIB or higher. 115 Patients underwent induction-therapy and 162 underwent primary surgery. Out of those 162 patients 158 were node positive $(\mathrm{pN}+)$ and accurately detected by PET-scan in 44 patients. CT-scan accurately detected $\mathrm{N}+$ in 65 patients, EUS in 112 patients. Fourteen patients with only peritumoral $\mathrm{N}+$ were identified by PET/CT/EUS in $2 / 6 / 8$ patients. Combining the 3 modalities sensitivity was $85.44 \%$; specificity $25.0 \%$. Overall 5-years-survival was $31.9 \%$. Patients with $>6$ positive nodes had a significantly worse 5 -years-survival $(22.7 \%)$ compared to $35.9 \%(p=0.0024)$.

Conclusions. In patients with locally advanced oesophageal cancer the low sensitivity of PET-scan 
could be significantly improved by adding CT and/or EUS. The routine preoperative use of these modalities should be encouraged to decrease false-positivity and delay in surgical treatment and to improve selection of node-positive patients that may benefit from neoadjuvant treatment.

\section{5}

\section{Perioperative Optimierung von Patienten mit Ösophaguscarzinom verringert periopertive Morbidität}

\section{S. E. Gabor, T. Niernberger, H. Rabl}

Abteilung für Chirurgie, Leoben, Austria

Grundlagen. Ca. 50\% der Patienten mit Ösophaguskarzinom zeigen bei Diagnosestellung bereits ein primär inoperables Tumorstadium. Studien $\mathrm{zu}$ präoperativer Radiochemotherapie ergaben eine Verbesserung des Überlebens, teils auch Verbesserung des krankheitsfreien Überlebens. Diese multimodalen Konzepte stellen für den Patienten jedoch eine enorme Belastung dar meistens gepaart mit ausgeprägter Malnutition. Malnutrition bedingt jedoch erhöhte perioperative Morbidität und damit verlängerter Rehabilitation sowie verlängerten Aufenthaltszeiten im Krankenhaus und damit wiederum erhöhten Kosten. Wir möchten zeigen, wie mit Hilfe eines periopertiven Optimierungskonzeptes diese Problematik verbessert werden kann.

Methodik. Seit 2009 wurden an unserer Abteilung 15 Patienten ( 3 weibl, 12 männl) im Rahmen eines solchen Konzeptes therapiert. Nach Durchführung der neoadjuvanten Radiochemotherapie wurde nach einer Erholungsphase von 6-8 Wochen die operative Sanierung (transhiatale oder transthorakale Ösophagektomie mit retrosternalem Magenhochzug) vorgesehen. Unser Optimierungskonzept enthält die Erfassung des Ernährungszustandes wenn notwendig Zufuhr von hochkalorischer Zusatznahrung, Durchführung einer Pharmakonutrition mit Omega 3 Fettsäuren sowie Glutamin 3 Tage präoperativ sowie Start der enteralen Ernährung 6 Stunden postoperativ.

Ergebnisse und Schlussfolgerungen. Im Rahmen dieses Konzeptes konnten wir die perioperativen Komplikationen sehr gering halten. Bei einem Patienten kam es zu einer Pneumonie, 2 Patienten mussten postoperativ beatmetet werden, 2 Patienten hatten eine Anastonoseninsuffizienz. Die Mortalität betrug $0 \%$.

\section{6}

\section{Retrospektive Analyse von 223 Patienten mit Kolorektalem Frühkarzinom}

\author{
P. Kogler ${ }^{1}$, D. Öfner ${ }^{2}$, R. Kafka ${ }^{1}$, J. Pratschke ${ }^{1}$, M. Zitt ${ }^{1}$ \\ ${ }^{1}$ Univ. Klinik für Viszeral-, Transplantations- und \\ Thoraxchirurgie, Medizinische Universität Innsbruck, \\ Innsbruck, Austria; ${ }^{2}$ Univ. Klinik für Chirurgie, Paracelsus \\ Medizinische Privatuniversität, Salzburg, Austria
}

Grundlagen. Die kurative Therapie des Kolorektalen Karzinoms basiert auf der Einhaltung chirurgisch-onkologischer Radikalitätsprinzipien bei gleichzeitig geringer Morbidität sowie Maximierung der Lebensqualität. Die Therapie des Kolorektalen Frühkarzinoms allerdings tendiert zunehmend in Richtung onkologisch-limitierter Techniken (Vollwandexzision, endoskopische Mukosaresektion, Polypektomie), was in der rezenten Literatur kontrovers diskutiert wird.

Methodik. Patienten $(n=223 ; \mathrm{m}: 127, \mathrm{w}: 96$, Mittel 65J), welche mit einem kolorektalen Frühkarzinom zwischen 1990-2010 an unserer Abteilung behandelt worden sind, werden bezüglich pathologischer Parameter, OP-Technik, lokoregionärem Rezidiv, Metastasierung sowie Patientenüberleben retrospektiv analysiert.

Ergebnisse. Histologisch fanden sich in $95 \%$ der Fälle Adenokarzinome, vornehmlich im Rektum (46,7\%), seltener im Sigma (19,8\%) und linken $(17,8 \%)$ bzw. rechten (15,7\%) Hemikolon. Insgesamt zeigten $47,5 \%$ high-risk Kriterien, 52,5\% waren low-risk Frühkarzinome. Zehn Patienten $(4,5 \%)$ waren bei Diagnosestellung bereits metastasiert. Primär wurden 120 Patienten $(53,8 \%)$ onkologisch radikal operiert, $26 \%$ der initial limitiert operierten Patienten $(46,2 \%)$ sekundär radikal nachreseziert. Lokalrezidive traten bei 7 Patienten (3\%; 6 nach limitierter OP-Technik) auf. Im Beobachtungszeitraum verstarben 55 Patienten (24,6\%); 9 (4\%) an Zweitmalignomen, 1 Patient $(0,44 \%)$ infolge des kolorektalen Frühkarzinoms. Kaplan-Meier-Überlebensanalysen werden präsentiert.

Schlussfolgerungen. Obwohl über ein Drittel der Patienten trotz hohem Anteil an high-risk Frühkarzinomen onkologisch-limitiert operiert wurden, zeigen sich durchaus gute Langzeit Ergebnisse: Somit ist es gerechtfertigt, unter strenger Indikationsstellung, diese Techniken anzuwenden.

\section{7}

\section{Management of second recurrence of colorectal carcinoma (CRC) liver metastases}

M. O. Biebl, C. Wolf, W. Mark, R. Öllinger, S. Schneeberger, M. Maglione, E. Laimer, J. Pratschke

Department of Visceral, Transplant and Thoracic Surgery, Medical University Innsbruck, Innsbruck, Austria 
Background. Aim of the study was to report the management of second recurrent hepatic CRC metastases.

Methods. Retrospective analysis of all consecutive patients undergoing liver resection for a second recurrence of CRC liver metastasis in a 7-year period. Of 109 patients undergoing resection for recurrent liver metastasis, 24 patients $(22.0 \%)$ were treated for a second tumor recurrence. Pre-, peri- and postoperative data are reported as total numbers (\%) or mean \pm standard deviation.

Results. Mean patient age at time of reoperation was $59 \pm 12$ years. One tumor nodule was present in $70.8 \%$, two nodules in $8.3 \%$ and three in $12.5 \%$. Preoperative chemotherapy was given in eigth patients (33.3\%). Preoperative blood testing revealed elevated tumor markers (CEA $56.77 \mathrm{ng} / \mathrm{ml} ; \quad$ Ca. $\quad 19-9 \quad 108.46 \mathrm{U} / \mathrm{ml} ; \quad$ AFP $16.95 \mathrm{IU} / \mathrm{ml}$ ). Mean preoperative liver function parameters were normal. Resection type was atypical (62.5\%), right (12.5\%), right extended (12.5\%), left (8.3\%) and mono-segment (4.2\%). Mean ICU time was $2.5 \pm 7.0$ days, total length of stay $14.8 \pm 9.9$ days. Postoperative complications occurred in $25 \%$. Postoperative chemotherapy was given to $41.7 \%$. With a mean follow-up of $19.7 \pm 22.3$ months, $58.3 \%$ of patients were free from recurrence.

Conclusions. Although recurrence rates are high, 2nd recurrent liver resection is meaningful in selected patients.

\section{8}

\section{Kolorektale Chirurgie in einem Bezirkskrankenhaus}

\section{T. K. Grißmann, T. Mayr, B. Spechtenhauser, B. Furtmüller}

Division of General Surgery, General Hospital Kufstein, Kufstein, Austria

Grundlagen. Zur Standortbestimmung in der kolorektalen Chirurgie im BKH Kufstein erfolgt in einer retrospektiven Aufarbeitung eine kritische Auseinandersetzung mit Komplikations- und Reoperationsraten als wichtiges Qualitätskriterium.

Methodik. Von $01 / 2004$ bis $12 / 2010$ wurden an unserer Abteilung 639 kolorektale Eingriffe (305 Frauen, 334 Männer, mittleres Alter 64 (18-93), mittlerer BMI von 25,9 (25,5 bei Frauen, 26,5 bei Männern), ASA 2,28 (1-4)) mit Anlage einer primären Darmanastomose oder Stomaanlage durchgeführt.

Ergebnisse. $77 \%$ der Eingriffe erfolgten am linken Hemikolon, $16 \%$ Zökum/Colon ascendens, $4 \%$ am Kolon transversum. Indikation zur Operation waren in 29,5\% maligne Erkrankungen, in 45,7\% Entzündungen, $19 \%$ waren Akuteingriffe. Die durchschnittliche Operationszeit betrug 140 Minuten, die mittlere Verweildauer lag bei 13 Tagen (2-90). Die häufigste Komplikationen in der Frühphase waren die Anastomoseninsuffizienz $(n=37)$ und
Infekte $(n=3)$, im weiteren Verlauf Hernien und andere wenige. Im beobachteten Zeitraum nahm die Anzahl der Eingriffe insgesamt stetig $\mathrm{zu}$, in 41 Fällen musste innerhalb von 14 Tagen eine Reoperation durchgeführt werden.

Schlussfolgerungen. Bei steigender Anzahl der kolorektalen Eingriffe im beobachteten Zeitraum hat sich dei Zahl der Komplikationen verringert, unseres Erachtens wegen der zunehmenden Frequenz und der Standartisierung der Eingriffe. Die Komplikationsrate liegt mit $5 \%$ im guten internationalen Vergleich.

\section{9}

The viennese experience: pancreatic metastasis from renal cell carcinoma

\section{G. Werba, P. Götzinger, M. Gnant, K. Sahora}

Department of Surgery, Medical University of Vienna, Vienna, Austria

Background. Pancreatic metastasis from any malignant tumor entity occurs quite rarely -2 to $11 \%$ of tumors found in the pancreas are secondary lesions. In most cases $(20 \%)$ the stomach states the primary malignancy site, however, renal cell carcinomas (RCCs) only represent $1 \%$ of the primi.

Methods. In this retrospective evaluation 20 patients treated at the General Hospital Vienna, from 1995 to 2007 , due to a malignant disease with metastases to the pancreas were analyzed. Data was obtained from the hospital information system. Fifteen patients ( 8 men, 7 women) with histologically verified metastases of clear cell RCCs could be identified.

Results. The mean age at diagnosis of the primary tumor was $60.3 \pm 7.8$ years. Secondary lesions could be found in the head $(n=7)$, body $(n=3)$ and tail $(n=5)$ of the pancreas $9.0 \pm 5.8$ years later, whereas one patient presented with a synchronous metastasis. All patients underwent surgical treatment for both pathologies with an average timespan of $9.2 \pm 5.2$ years between their occurrences. There was no statistical significance comparing the 10 -year-survival of men (50\%) and women (80\%).

\section{0}

Predicting the prognosis of operated, non-functioning neuroendocrine carcinoma of the pancreas - which classification should we use?

\section{S. Thalhammer, S. Stättner, M. Klimpfinger, J. Karner, F. Sellner}

Kaiser Franz Josef Spital, Vienna, Austria 
Background. Non-functioning neuroendocrine carcinomas of the pancreas (nfnepC) are a rare tumorentity, therefore only a few studies compare the two current classification systems (WHO-Classification in well and poorly differentiated carcinomas $v s$. the classification by TNM and grading system). This prompted us to evaluate, compare and assess the prognostic significance of these two systems in a homogenous group of curative resected nfnepC.

Methods. Eighteen nfnepC were retrospectively analyzed for differences in survival.

Results. There was a correlation between pT (p .026), respectively pM categories (.016) and survival. $\mathrm{G}$ categories and length of survival were closely correlated ( $p$.0036). Disease stages I-IV had a significant effect on survival ( $p$.051). The WHO-classification in well and poorly differentiated carcinomas proved to be the most conclusive predictive factor ( $p$.0009). Subgroups with significantly different prognoses determined by histological grade were present within disease stage II.

Conclusions. The retrospective analysis showed a good correlation between survival and pT, pM, tumor stage, G categories and WHO classification in well and poorly differentiated carcinomas. Including histological differentiation in the staging system or carrying it out separately in well and poorly differentiated carcinomas, could enhance the predictive potential of TNM-based disease stages (Accepted by JSO for publication).

\section{1}

\section{Mammametastase eines Osteosarkoms: Ein sehr seltener Fall}

\section{Hackl, M. Dünser, J. Pratschke}

Univ. - Klinik für Viszeral-, Thorax- und Transplantationschirurgie, Innsbruck, Austria

Grundlagen. Osteosarkome sind die häufigste bösartige Erkrankung des Knochens und metastasieren hauptsächlich in die Lungen. Mammametastasen sind dagegen sehr selten bzw. kaum in der Literatur beschrieben.

Methodik. Wir möchten den Fall einer 35 jährigen jungen Frau vorstellen.

Ergebnisse. Diese Patientin, welche in der Vorgeschichte wegen eines Osteosarkoms am Unterschenkel behandelt wurde, wurde wegen einer Mammametastase von uns operiert. Es erfolgte 2009 eine Exstirpation mit histologisch breiten gesunden Resektionsrändern. 2011 war eine weitere Operation im Sinne einer einfachen Mastektomie aufgrund einer neuerlichen diesmal diffus ausgebreiteten Metastasierung notwendig. Simultan wurde die Brust mit einer Silikonprothese rekonstruiert.

Schlussfolgerungen. Obwohl Mammametastasen eines Osteosarkoms sehr selten sind, sollte man die Möglichkeit einer solchen Differentialdiagnose in Erwä- gung ziehen, falls man bei Patientinnen, die bereits in der Anamnese ein Osteosarkom aufwiesen, eine suspekte Raumforderung in der Brust feststellt.

\section{2}

\section{Spätmanifestation eines Merkelzellkarzinoms - Nebennierenmetastase 8 Jahre nach primärer Tumorexstirpation}

\section{Aspalter ${ }^{1}$, D. Neureiter ${ }^{2}$, H. Waclawiczek ${ }^{1}$, D. Öfner-Velano ${ }^{1}$, K. Emmanuel ${ }^{1}$ \\ ${ }^{1}$ Paracelsus Medizinische Privatuniversität, Department für Allgemeinchirurgie, Salzburg, Austria; ${ }^{2}$ Paracelsus Medizinische Privatuniversität, Department für Pathologie, Salzburg, Austria}

Merkelzellkarzinome sind seltene, aggressiv-wachsende, neuroendokrine Tumoren der sonnenexponierten Haut. Fernmetastasen treten in etwa 1-7\% der Fälle auf und werden in den meisten Fällen im ersten Jahr nach primärer Tumorexstirpation diagnostiziert.

Wir berichten den Fall einer 77 jährigen Patientin, bei welcher 8 Jahre nach primärer Exstirpation eines Merkelzellkarzinoms der Wange ein großer Tumor der rechten Nebenniere diagnostiziert wurde. Immunhistochemische Analysen des Präparats zeigten eine Positivität für Pan-Cytokeratin AE1/AE3, Cytokeratin 7 und 8/18 und EMA (Epithelial membrane antigen). $\mathrm{Zu}-$ sätzlich zeigte sich sowohl eine starke Co-Expression von Synaptophysin, NSE (Neuron specific enolase) und Chromogranin A als auch eine Kernfärbung für TTF-1 (Thyroid transcription factor-1). Dieser Befund sprach in erster Linie für eine Metastase eines neuroendokrinen Karzinoms der Lunge. Im Rahmen der weiteren Primumsuche (Computertomographie des Thorax und Abdomens, DOPASCAN, Gastroskopie, Koloskopie) fand sich kein Hinweis auf einen Lungentumor oder Tumor eines anderen Organs. Deshalb wurde in weiterer Folge das Präparat des Merkelzellkarzinoms re-evaluiert. Obwohl der Hauttumor eine Negativität für TTF-1 aufwies, zeigten sich alle anderen immunhistochemischen Marker gleichmäßig exprimiert. Demzufolge ist die Diagnose einer Nebennierenmetastase eines vor 8 Jahren resezierten Merkelzellkarzinoms als wahrscheinlichste Diagnose in Betracht zu ziehen. Nach einer Nachbeobachtungszeit von 7 Monaten ist die Patientin wohlauf und ohne Tumorprogression. 


\section{3}

\section{De novo bronchial cancer following kidney transplantation}

\section{Maglione, F. Augustin, C. Boesmueller, M. Biebl, J. Bodner, P. Lucciarini, J. Pratschke, T. Schmid}

Department of Visceral, Transplant and Thoracic Surgery, Innsbruck Medical University, Innsbruck, Austria

Background. Chronic immunosuppression is associated with a higher cancer incidence. From these, bronchial cancer (BC) is associated with the highest mortality rate. Aim of this study was to evaluate incidence and outcome of patients developing BC following single or combined kidney-pancreas/liver transplantation.

Methods. Patients transplanted between 1996 and 2010 were analyzed retrospectively.

Results. Among 1761 patients, 20 (1.14\%, 5 females) developed BC. 16 of these had a kidney, 3 a simultaneous pancreas-kidney and 1 patient a simultaneous liver-kidney transplant. 19 (95\%) patients admitted smoking. Median age at transplantation was 56 (range 39-65) years. Pre-transplant chest X-rays were without abnormal findings. All but one patient received calcineurin-based immunosuppression. Median interval from transplantation to tumor diagnosis was 57 (3-205) months. BC was diagnosed at UICC stage IV in 13 patients (65\%, 1 SCLC). Seven BCs were diagnosed and resected at UICC stage I. Three patients experienced complete response, in 2 disease progressed and 2 died of postoperative complications. With a median follow up of 10.7 (2-39) months, $\mathrm{BC}$ recurred in $28.6 \%$

Conclusions. Due to poor prognosis of BC post transplantation intensive educational training, special screening and therapeutic strategies are necessary for transplant patients with a history of smoking.

\section{Arbeitsgemeinschaft für Minimal Invasive Chirurgie: Varia}

\section{8}

\section{Erfahrungen mit dem transoralen Zugang in der endoskopischen Schilddrüsenchirurgie}

\author{
K. Witzel'1, F. G. Messenbäck ${ }^{2}$, T. Benhidjeb ${ }^{3}$ \\ ${ }^{1}$ Minimal Invasiv Center, Hünfeld, Germany; ${ }^{2}$ Kardinal \\ Schwarzenberg'sches Krankenhaus, Schwarzach \\ im Pongau, Austria; ${ }^{3}$ Universitätsklinikum, \\ Hamburg-Eppendorf, Germany
}

Endoskopische und minimal-invasive Operationstechniken an der Schilddrüse werden zunehmend nachgefragt und durchgeführt. Die beschriebenen extracollaren Zugänge sind mit einem erhöhten Zugangstrauma zugunsten eines besseren kosmetischen Ergebnisses verbunden. Die Möglichkeit der endoskopischtransoralen Schilddrüsenresektion wurde nach Erarbeitung der anatomischen Grundlagen in präklinischen Studien am Tier und an der Leiche von uns 2008 erstbeschrieben. Wegen nachfolgender Publikationen mit Darstellung chirurgisch inadäquater endoskopisch transoraler Resektionen führten wir in Anwesenheit von Vertretern der chirurgischen Gesellschaften den klinischen Versuch einer transoralen Hemithyroidektomie durch. Wir führten zunächst die endoskopische Mobilisation des Schilddrüsenlappens durch. Die Erfahrung des OP-Teams mit dieser Technik liegt bei über 150 Lobektomien. Hiernach folgte der transorale Zugang. Aufgrund anatomischer Limitationen konnte jedoch die vollständige Bergung des Präparates nicht transoral erfolgen, so dass eine collare Schnitterweiterung zur Bergung erforderlich wurde. Bei neuen Verfahren ist die Patientensicherheit neben dem adäquaten Operationsergebnis entscheidend. Zur Erreichung dieses Ziels haben wir ein Hybridverfahren gewählt, um den klinisch-experimentellen Anteil zu reduzieren. Bei der Planung und Durchführung sollte immer auf eine maximale Transparenz unter Einbeziehung der Fachgesellschaften geachtet werden. Ein alleiniges Ethikvotum könnte fachlich nicht ausreichend sein. Bei den endoskopischen Verfahren scheint der transorale Zugang langfristig der Günstigste, denn der vereint ein minimales Zugangstrauma mit einen guten kosmetischen und narbenfreien Ergebnis.

\section{9}

\section{Clear determination of adrenal tumor} size reduces the conversion rate

\section{A. Pereyaslov, Y. Havrysh \\ Medical University, Lviv, Ukraine}

Adrenal tumor size often determines the possibility of its laparoscopic removing. With the increasing of the tumors' size arising the technical difficulties and probability of malignant character of tumor. Due to that the pre-operative determination of clear size of adrenal tumor may reduce the rate of conversion during laparoscopic adrenalectomy.

The pre-operative tumors' size determined by CT and real histological size were compared in 54 patients operated with adrenal tumors. All patients were divided on two groups: first group formed 23 patients in which preoperative tumor size measured by CT results only, second group formed 31 patients in which D. Linos formula was applied. The mean size of tumor in patients of the first group was $5.2 \pm 0.3 \mathrm{~cm}$ with the real size $-6.6 \pm 0.3 \mathrm{~cm}$ 
$(p<0.05)$ while in patients of the second group pre-operative size was $5.9 \pm 0.4 \mathrm{~cm}$ with the histological size -6.2 $\pm 0.3 \mathrm{~cm}$ ( $p>0.05)$. Among patients of the first group, conversion due to the bigger tumor size was performed in $4(17.4 \%)$ patients and in the second group the rate of conversion was $6.5 \%$ ( 2 patients).Thus, the applying the Linos formula for the pre-operative measuring of the adrenal tumor size decrease the rate of conversion during laparoscopic adrenalectomy.

\section{0}

\section{Laparoskopische Operationen: schmerzarm?}

\section{Pilz ${ }^{1,2}$, W. Wayand ${ }^{1,2}$}

${ }^{1}$ AKH Linz/Chirurgie II, Linz, Austria; ${ }^{2}$ Ludwig Boltzmann Institut für operative Laparoskopie, Linz, Austria

Grundlagen. Nach der Zertifizierung zur „Schmerzfreien Chirurgie“ im August 2009 als erste Abteilung in Österreich müssen wir durch regelmäßige Patientenbefragungen die Ergebnisqualität kontrollieren und für eine stetige Verbesserung sorgen.

Methodik und Ergebnisse. Wir haben alle operierten Patienten im Februar 2008, Februar 2009 und März 2010 in den ersten 2 postoperativen Tagen nach ihren Schmerzen und schmerzbedingten Beeinträchtigungen befragt. Dabei hat sich der Anteil an schmerzfreien Patienten (VAS maximal 3) am Operationstag von 2008 auf 2009 von $34,2 \%$ auf $41,1 \%$ verbessert. Im Jahr 2010 konnte eine nochmalige Verbesserung auf $43,7 \%$ erreicht werden. Im März 2010 wurde zusätzlich zu den Schmerzen auch die durchgeführte Operation auf dem Fragebogen festgehalten. Damit konnten die 33 Patienten (25,2\% der ausgewerteten Fragebögen), die im Beobachtungszeitraum auf den Bettenstationen nicht durchgehend schmerzfrei waren, weiter analysiert werden. Es zeigte sich dabei, dass 10 von ihnen eine laparoskopische Operation hinter sich hatten.

Schlussfolgerungen. Patienten nach laparoskopischen Operationen bedürfen auf der Normalstation erhöhter Aufmerksamkeit, da sie mit der Basisschmerzmedikation gelegentlich nicht auskommen.

\section{1}

\section{SILS for resection of a large liver adenoma}

\section{W. Mark, A. Klaus, M. Biebl, J. Pratschke}

Department of Operative Medicine, Innsbruck, Austria

Background. Liver surgery has adopted laparoscopic techniques in order to minimize abdominal wall trauma.
We describe resection of a large mass in the liver using non stapling SILS technique.

Methods. A female patient $(25$ yrs, $\mathrm{BMI}=30.4)$ presented with an incidentially detected tumor of the left liver lobe. Apart from GERD which was successfully controlled by PPI no further symptoms were present. Due to rapid progression in size from 6 to $11 \mathrm{~cm}$ in diameter within 6 months indication for resection of the suspected FNH or adenoma (CT scan, MRI) was set.

Results. A $40 \mathrm{~mm}$ gelport was introduced via the umbilicus. No additional trocar was required. Parenchyma was dissected without any stapler and blood loss was $<30 \mathrm{ml}$. In order to retrieve the specimen via the umbilicus it had to be dissected intracorporally into smaller pieces within an endobag. Total operating time was $100 \mathrm{~min}$. Histology revealed hepatocellular adenoma.The patient could be discharged after an uneventfull course on pod 4.

Conclusions. Resection of a giant liver tumor is feasible by SILS technique which minimizes abdominal wall trauma and may contribute to wider guidelines for indication of resecting liver adenomas.

\section{2}

Die onkologische Lymphknotendissektion im Rahmen der VATS-Lobektomie beim nicht kleinzelligen Bronchuskarzinom

\section{J. Bodner, F. Augustin, T. Schmid}

Univ.-Klinik für Chirurgie, Innsbruck, Austria

Grundlagen. Speziell in Europa findet der VATS (Video Assisted Thoracic Surgery) Zugang bei der chirurgischen Therapie des NSCLC (non small cell lung cancer) im Frühstadium nur sehr langsam zunehmende Akzeptanz. Trotz erwiesener Vorteile für die Patienten hinsichtlich rascherer Rehabilitation und besserer Toleranz adjuvanter Therapieformen halten sich verbreitet Bedenken in onkologischer Hinsicht. Dabei steht vor allem die technische Machbarkeit einer adäquaten mediastinalen Lymphknotendissektion zu Diskussion.

Methodik. Anhand von intraoperativen Videosequenzen wird die Technik der thorakoskopischen Lymphknotendissektion der mediastinalen $(2 / 4,7,8,9)$, hilären (10) und intrapulmonalen (11) Lymphknotenstationen Stationen demonstriert.

Ergebnisse. Die mediastinale Lymphknotendissektion im Rahmen von onkologischen Lobektomien wegen eines nicht-kleinzelligen Bronchuskarzinoms ist mittels VATS Zugang vollständig und sicher durchführbar.

Schlussfolgerungen. Die onkologische Lymphknotendissektion mittels VATS kann hinsichtlich Radikalität gleichwertig zu jener beim offenen Zugang durchgeführt werden. Daher erscheint eine Anwendung des VATS Zugang auch bei höheren Stadien des NSCLC als dem Stadium I gerechtfertigt. 


\section{Arbeitsgemeinschaft für}

\section{Hernienchirurgie: Leistenhernie}

\section{4}

\section{Outcome of 356 single incision laparoscopic groin hernia repairs (SIL-TAPP)}

\section{W. Brunner, J. Schirnhofer, R. Frass, N. Waldstein, K. Pimpl, C. Mittermair, C. Obrist, H. Weiss \\ KH Barmherzige Brüder, Salzburg, Austria}

Background. Laparoscopic hernia repair has proven to achieve excellent long term results. Herein we report a series of TAPP repair utilizing solely the transumbilical laparoscopic route.

Methods. A total of 290 patients (Mean \pm SD age $55 \pm$ 15 years, BMI $25 \pm 3 \mathrm{~kg} / \mathrm{m}^{2}$ ) were operated on unilateral (224) or bilateral (66) TAPP. Recurrent hernia were present in 37 patients on one side, in 4 on both sides (13\% of all).

Results. All but 18 procedures (5\%) could be completed without adding an additional trocar. Operative time yielded (Mean) $52 \mathrm{~min}$ (49 min unilateral and $67 \mathrm{~min}$ bilateral). One intraoperative lesion of the bladder after prostatectomy needed laparoscopic suturing. Two patients required revisional surgery on day 1 for wound hematoma. Low pain score (0-2) was measured in 230, moderate (3-5) in 51, and severe $(>5)$ in $9(3 \%)$ patients on the first day. Patients were discharged within three days postoperatively. Umbilical wound infection occurred in 4 patients treated conservatively. Within a follow-up of up to 24 months seven patients (1.9\%) were readmitted for recurrent hernia, 3 for umbilical hernia (1\%).

Conclusions. Single access transumbilical TAPP is safe and feasible for unilateral and bilateral groin hernia with a favourable rate of complication or recurrence.

\section{5}

\section{SIL-TAPP versus Lichtenstein repair: a matched-pairs analysis}

\section{Obrist, W. Brunner, J. Schirnhofer, K. Pimpl, C. Mittermair, R. Frass, N. Waldstein, H. Weiss}

Chirurgie, Salzburg, Austria

Background. The single incision lapraroscopic (SIL) hernia repair was introduced as a safe and feasible technique. A retrospective matched-pairs analysis SIL-TAPP $v s$. Lichtenstein repair is demonstrated.

Methods. A total number of 160 patients with primary unilateral inguinal hernia underwent surgery between
$11 / 2008$ and $12 / 2009$. The patients were devided into two groups (Group A: SIL-TAPP $n=80,75 \mathrm{~m} / 5 \mathrm{f}$, Mean age $53 \mathrm{a}$, Mean BMI $25.0 \mathrm{~kg} / \mathrm{m}^{2}$; and Group B: Lichtenstein repair $n=80,75 \mathrm{~m} / 5 \mathrm{f}$, Mean age $64 \mathrm{a}$, Mean BMI $25.8 \mathrm{~kg} / \mathrm{m}^{2}$ ). Perioperative parameters and postoperative follow-up including clinical examiniation and a questionnaire were documented.

Results. Operative time was significantly shorter in the Lichtenstein group $(p<0.01)$ whereas wound infections were observed only in this group. No intraoperative complication occurred in both groups. Postoperative pain was neglectable in $81 \%$ of Group A and $55 \%$ of Group B patients. After a follow-up of at least one year recurrent hernia was found in 4 and 8 patients of group A and B, respectively. Patient satisfaction was optimal in all but the patients with recurrencies.

Conclusions. This study demonstrates that the shorter procedural time for Lichtenstein repair in single site hernia repair is balanced by a favourable outcome using the SIL-TAPP technique regardless of the cosmetic result.

\section{6}

\section{MILS vs. SILS bei der TAPP Technik - Ergebnisse einer prospektiv randomisierten Studie}

\author{
C. Hollinsky, S. Hollinsky, P. Razek, P. Partri, \\ C. Beran, A. Tuchmann
}

SMZ Floridsdorf, Surgery, Vienna, Austria

Grundlagen. Bei der TAPP Technik stellt sich die Frage, ob mit einem Zugang (SILS) oder durch drei Miniincisionen (MinimalIncisionLaparoscopicSurgery - MILS) für Patienten bessere Ergebnisse zu erzielen sind.

Methodik. An unserer Abteilung werden Patienten mit ein- und beidseitiger Leistenhernie Stadium I-II nach Schumpelick mit der TAPP Technik prospektiv randomisiert mit SILS oder MILS behandelt. Ausgewertet werden Operationszeit, intra- und postoperative Komplikationen, postoperative Schmerzen, Janda-Kriterien, Narbenhernien sowie das kosmetische Ergebnis.

Ergebnisse. Bisher wurden 80 Patienten in die Studie eingebracht, wobei mit $41 \mathrm{~min}$. vs. $79 \mathrm{~min}$. ein signifikanter Unterschied in der Operationszeit zugunsten der MILS Technik vorlag. Als intraoperative Komplikation kam es zu einer Blutung aus der A. epigastrica inf. in der SILS Gruppe, postoperative Komplikationen waren in beiden Gruppen gering und ohne signifikanten Unterschied. Sowohl bei den Kriterien nach Janda als auch bei den postoperativen Schmerzen waren signifikante Vorteile der MILS Technik zu erkennen. Das kosmetische Ergebnis war in beiden Gruppen für die Patienten sehr zufriedenstellend. Narbenhernien sind derzeit noch keine aufgetreten.

Schlussfolgerungen. Bei den vorliegenden Ergebnissen lassen sich deutliche Vorteile der MILS Technik 
bezüglich Operationszeit, Janda Score und postoperative Schmerzen erkennen.

\section{7}

Der klinische Befund einer Leistenhernie der Schwangeren - wie sollen wir damit umgehen?

\section{F. Mayer, M. Lechner, D. Öfner-Velano}

PMU Paracelsus Medizinische Privatuniversität, Salzburg, Austria

Grundlagen. Wird ein Chirurg mit der Fragestellung der präpartalen Sanierung eines Leistenhernienbefundes konfrontiert, ist bislang die Frage, wann bzw. ob dieser Befund präpartal operativ versorgt werden soll, in der Literatur umstritten diskutiert. Bezüglich Diagnostik und Therapie gibt es bislang keine einheitlichen Empfehlungen.

Methodik. In einer prospektiven klinischen Studie wurden 9 Schwangere mit dem Befund einer schmerzhaften Schwellung in der Leiste klinisch und mittels Farbdopplersonographie (FDS) untersucht. 4 Wochen postpartal und innerhalb eines Jahres wurden die Patientinnen kontrolliert.

Ergebnisse. Keine der Patientinnen zeigte den Befund einer Leistenhernie, es zeigte sich in allen Fällen eine variköse Dilatation der Venen entlang des Lig. teres uteri. Es wurde keine Patienten wegen des vermeintlichen Leistenhernienbefundes operiert. Alle Schwangerschaften und Entbindungen verliefen unkompliziert, bei 1/9 wurde eine Sectio caesaria mit dem (widerlegten) Vermutungsbefund einer Leistenhernie indiziert. Die Leistenbeschwerden sistierten bei allen Patienten nach der Entbindung.

Schlussfolgerungen. Die Durchführung einer FDS ist zur Diagnosesicherung bei einem vermeintlichen Leistenhernienbefund der Schwangeren zu fordern. Im Falle varikös dilatierter Venen entlang des Lig. teres uteri ist eine chirurgische Intervention nicht indiziert. Unserer Erfahrung nach stellt dieser Befund auch keinen prädisponierenden Faktor für die spätere Entwicklung einer Leistenhernie dar.

\section{8}

\section{Transporöse Netzfixierung mit Fibrinkleber in einem experimentellen Modell der Sprühanwendung}

\author{
J. Brand ${ }^{1}$, A. Petter-Puchner ${ }^{1,2}$, S. Gruber-Blum ${ }^{1,2}$, \\ K. Mika ${ }^{1}$, R. H. Fortelny ${ }^{1,2}$, H. Redl $^{1}$ \\ ${ }^{1}$ Ludwig Boltzmann Institut für experimentelle und klinische \\ Traumatologie, Wien, Austria; ${ }^{2} 2$. Chirurgie, Wilhelminenspital, \\ Wiener Krankenanstaltenverbund, Wien, Austria
}

Grundlagen. Die Anwendung von Fibrinkleber stellt eine weit verbreitete Methode zur Netzfixierung, sowohl in der offenen als auch in der laparoskopischen Hernienchirurgie dar. Derzeit gibt es kaum Studien zur Kleberverteilung unter Berücksichtigung der Netzeigenschaften. Verschiedene Einflussfaktoren, wie Sprühabstand und -druck, die Thrombinkonzentration des Klebers und das Netzdesign, spielen für die Kleberallokation eine wichtige Rolle.

Methodik. Drei verschiedene Netze (Porengröße 1-4 mm) wurden in einer vertikalen Versuchsanordnung mit jeweils 0,4 ml Fibrinkleber (Baxter Biosciences, Wien, Österreich), in Darreichungsformen zu 4 und 500 I.E. Thrombin $(n=10)$, aus den Abständen $5-8 \mathrm{~cm}$ bei 1,5 bar besprüht. Die relative Kleberverteilung auf den Netzen wurde evaluiert und die Ergebnisse miteinander verglichen. Darüber hinaus wurden die Auswirkungen eines unterbrochenen Sprühvorgangs auf die Netzstrukturen untersucht.

Ergebnisse. Sprühabstände zwischen 5 und $8 \mathrm{~cm}$ wiesen eine homogene Fibrinkleberverteilung auf. Die Verwendung von niedrigen Thrombinkonzentrationen verursachte zusätzliche Verluste, welche auf die langsamere Polymerisationsgeschwindigkeit des Klebers zurückzuführen waren. Unterschiede bezüglich der Fibrinkleberverteilung und der Netzporengröße konnten nachgewiesen werden. Es zeigte sich, dass die Netzstruktur eine wesentliche Rolle bei der Kleberverteilung spielt.

Schlussfolgerungen. Es waren keine Unterschiede zwischen kontinuierlicher und diskontinuierlicher Fibrinkleberapplikation nachweisbar. Die verwendete Gesamtmenge sollte bei handelsüblichen Netzen $0,1 \mathrm{ml} / \mathrm{cm}^{2}$ nicht überschreiten, um einen Verschluss von Netzporen zu vermeiden.

\section{9}

\section{Latrogenic nerve damage due to laparoscopic hernia mesh fixation}

\section{Poglitsch ${ }^{1}$, M. Stoiber ${ }^{2,3}$, H. Schima ${ }^{2,4}$, G. Prager ${ }^{1}$, E. Rieder ${ }^{1}$ \\ ${ }^{1}$ Department of Surgery, Medical University of Vienna, Vienna, Austria; ${ }^{2}$ Center for Medical Physics \& Biomedical Engineering, Medical University of Vienna, Vienna, Austria; ${ }^{3}$ LBC for Cardiovascular Research, Vienna, Austria; ${ }^{4}$ Department of Thoracic Surgery, Medical University of Vienna, Vienna, Austria}

Background. Mesh fixation devices can lead to significant postoperative pain after laparoscopic ventral hernia repair due to potential nerve injury. The exact mechanism of iatrogenic nerve damage caused by penetrating tacks however, remains unclear. This study was designed to compare different fixation anchors regarding their potential to violate nerve integrity.

Methods. Porcine abdominal wall nerves were harvested and sealed in silicone about $2 \mathrm{~mm}$ deep. Three 
different fixation devices (Tack I: titanium helical tack; Tack II: absorbable PGLLA tack; Tack III: absorbable PDLLA fastener) were applied towards the embedded nerve. Tack application was videotaped and pictures were taken thereafter. Nerve damage was categorized into groups of no nerve trauma $(\mathrm{N})$, tangentially injured nerve (T), perforated nerve (P), and complete loss of continuity.

Results. Metal helical tacks (Tack I, $n=14$; P: $43 \%$; T: $50 \%$; N: $7 \%$ ) induced a higher rate of nerve perforations when compared to the absorbable Tack II $(n=10$; P: $20 \%$, T: $80 \%, \mathrm{~N}: 0 \%)$, or Tack III $(n=7 ; \mathrm{P}: 28.5 \%, \mathrm{~T}: 43 \%, \mathrm{~N}$ : $28.5 \%$ ), although differences were not significant. No complete loss of nerve continuity was observed throughout the experiments.

Conclusions. The new generation of absorbable tacks might be less likely to cause severe nerve perforations.

\section{0}

\section{Prospective, randomized-controlled trial comparing postoperative pain after open and minimal invasive inguinal hernia repair}

\section{F. Aigner ${ }^{1}$, F. Augustin ${ }^{1}$, C. Kaufmann ${ }^{1}$, A. Schlager ${ }^{2}$, J. Pratschke ${ }^{1}$, T. Schmid ${ }^{1}$ \\ ${ }^{1}$ Univ.-Klinik für Visceral-, Transplantations- und Thoraxchirurgie, Innsbruck, Austria; ${ }^{2}$ Univ.-Klinik für Anästhesie und Intensivmedizin, Innsbruck, Austria}

Background. The aim was to compare postoperative pain between the open tension-free plug and patch (PP) technique and the totally extraperitoneal patch (TEP) hernioplasty.

Methods. One hundred and sixty-two male patients with unilateral inguinal hernia were randomized to undergo PP and TEP from 2005 to 2009. Pain assessment was conducted by using the numerical rating scale (NRS) and the McGill Pain Questionnaire preoperatively, 6, 12 and 24 months postoperatively. All patients obtained same analgesics and documented pain in a NRS based 4-week-diary.

Results. Of the 162 patients 79 underwent TEP and 83 PP. Mean follow-up was $2.9 \pm 1.2$ years. One recurrent hernia was observed in the TEP group. Mean preoperative NRS score was 2 and 2, 0.3 and 0.4 at 6 months, 0.1 and 0.3 at 12 months, 0.2 and 0.1 at 24 months postoperatively in the PP and TEP groups respectively $(p>0.05)$. Data from the 4-week-pain diaries revealed no significant difference in pain intensity in the first two weeks postoperatively (VAS 2-4, $p>0.05$ ). Patients in the PP group required more additional analgesics on day four and five postoperatively ( $p=0.037$ and 0.015 , respectively).

Conclusions. Our data demonstrate no significant differences concerning postoperative pain between tensionfree PP and TEP hernia repair.
Chirurgische Forschung IV: Chirurgische Innovationen

\section{1}

\section{Isolierung und Kultivierung Schwann'scher Zellen aus humanen peripheren Nerven}

\section{G. Weigel ${ }^{1}$, K. Geier ${ }^{1}$, A. Kuess ${ }^{2}$, A. Saxena ${ }^{2}$, S. Spendel ${ }^{1}$}

${ }^{1}$ Uinversitätsklinik für Chirugie, Klinische Abt. Plastische, Ästhetische und Rekonstruktive Chirurgie, Graz, Austria; ${ }^{2}$ Universitätsklinik für Kinder- und Jugendchirurgie, Klinische Abt. allgemeine Kinder- und Jugendchirurgie, Graz, Austria

Grundlagen. Den Goldstandard bei der Überbrückung peripherer Nervenläsionen beim Menschen stellt die Transplantation autologer Nerven dar, dessen Entnahme zu Sensibilitätsverlust führt. Deshalb wird an der Entwicklung von artifiziellen Implantaten, besiedelt mit Schwann'schen Zellen, geforscht, die als Nervenleitschiene dienen und das axonale Wachstum der peripheren Nerven fördern. Voraussetzung dafür sind reine Schwannzell-Kulturen. Unsere Arbeitsgruppe beschäftigt sich nun seit mehr als 1 Jahr mit der Isolierung und Kultivierung von Schwann'schen Zellen aus humanen peripheren Nerven.

Methodik. Humane periphere Nerven („Abfall“ bei Humaneingriffen) wurden unter sterilen Bedingungen präpariert und auf 6-Well-Kulturplatten mit Nährmedium aufgebracht. Nachdem die aussprossenden Zellen einen konfluenten Monolayer um die Nervenstücke gebildet hatten, wurden diese in neue Kulturplatten weiterpassagiert. Die Identifikation der Schwannzellen erfolgte mittels S100-Färbung.

Ergebnisse. Erste Zellaussprossungen konnten in den Primärkulturen bereits nach 3-7 Tagen festgestellt werden, jedoch waren in diesen Kulturen Fibroblasten die dominierenden Zellen. Durch mehrmaliges Passagieren konnte der Fibroblastenanteil jedoch deutlich gesenkt und annähernd reine Schwannzell-Kulturen hergestellt werden. Die gesamte Kultivierungsdauer betrug ca. 4-6 Wochen, bis eine ausreichend große und reine Zellpopulation für die Aufbringung auf Polymere zur Verfügung stand.

Schlussfolgerungen. Mit dem Erhalt reiner Schwann Zell Kulturen soll nun die Besiedelung von Conduits erfolgen. 


\section{2}

\section{Establishment of a sound model of persistent hindlimb ischemia allows reduction of experimental animal use}

\author{
A. Kocher ${ }^{1}$, K. Albrecht ${ }^{2}$, C. Krapf ${ }^{3}$, M. Kofler ${ }^{4}$ \\ N. Bonaros ${ }^{5}$, M. Maruszewski ${ }^{1}$, B. Schlechta ${ }^{6}$, \\ W. Schgoer ${ }^{7}$, M. Grimm ${ }^{5}$, S. Aharinejad' ${ }^{1}$, \\ D. Wiedemann ${ }^{8}$
}

${ }^{1}$ Department of Cardiac Surgery, Vienna Medical University, Vienna, Austria; ${ }^{2}$ Department of Internal Medicine I, Innsbruck Medical University, Innsbruck, Austria; ${ }^{3}$ Department of General and Transplant Surgery, Innsbruck Medical Univeristy, Innsbruck, Austria; ${ }^{4}$ Department of General and Transplant Surgery, Innsbruck Medical University, Innsbruck, Austria; ${ }^{5}$ Department of Cardiac Surgery, Innsbruck Medical University, Innsbruck, Austria; ${ }^{6}$ Department of Gynaecology and Obstetrics, Vienna Medical University, Vienna, Austria; ${ }^{7}$ Department Internal Medicine I, Innsbruck Medical University, Innsbruck, Austria; ${ }^{8}$ Department of Cardiac Surgery, Vienna Medical University and University Clinic of Cardiac Surgery, Innsbruck Medical University, Vienna, Innsbruck, Austria

Background. Poorly designed and conducted experiments lead to a waste of scientific resources.99\% of the rodent models of hindlimb ischemia reported in the scientific literature describe either ligation or exicision of a short segement of the common femoral artery.The purpose of the current study was to test the spontaneous revascularisation potential employing this model in three different species.

Methods. Mice, rabbits and athymic nude rats were investigated. A part of the femoral artery was excised from $5 \mathrm{~mm}$ in the mouse model up to $2 \mathrm{~cm}$ in the rabbit model exactly according to the literture. Perfusion was tested by scan or laser doppler preoperatively and at weekly intervals for one month.

Results. The perfusion in all hindlimbs returned to baseline levels and the results were not statistically significant from the contralateral leg, which served as control. This was achieved without any intervention or injection of growth factors, stem cells or application of any other magic bullet.

Conclusions. The ischemic hindlimb recovers to full extent to baseline levels within 4 weeks after femoral artery excision without difference across 3 species. We plea to employ and publish only meaningful, functional models, in order to reduce the number of experimental animals used.

\section{3}

\section{Ösophagus Tissue Engineering: Etablierung einer erfolgreichen Methode zur Gewinnung von Gewebe und in-situ- Implantation im fetalen Schafmodell}

\author{
A. Saxena, H. Ainoedhofer, H. Hader, M. Höllwarth \\ Medizinische Universität Graz, Graz, Austria
}

Grundlagen. Das Ziel dieser Studie ist es, im Rahmen unserer Forschungstätigkeit des Speiseröhren-Tissue Engineerings, die Herstellung und Entwicklung von vaskularisierten, tubulären Konstrukten am fetalen Schafmodell in-situ zu untersuchen.

Methodik. Es wurden Biopsien der Speiseröhre von fetalen Austrian-Mountain-Schafen zwischen dem 79120 Schwangerschaftstag entnommen. Die gewonnenen Zellen wurden als „Esophagus organoid units“ (EOU) auf unterschiedlichen Polymeren kultiviert. Um eine tubuläre Struktur ähnlich der Speiseröhre zu erhalten, wurden die besiedelten Polymere über Stents genäht und in das Omentum (in-situ) implantiert. Nach der Geburt konnten die Polymere entnommen und histologisch untersucht werden.

Ergebnisse. Die Entnahme von fetalen Biopsien sowie das Implantieren der Polymere in das Bauchfell waren erfolgreich. Die Umwickelung des Konstruktes mit omentalem Gewebe gewährleistete Gefäßwachstum um und in das Implantat, sowie entlang der äußeren Polymerschicht. Nach Entfernung der Stents zeigte sich eine tubuläre Struktur mit offenem Lumen ähnlich der Speiseröhre. Die histologischen Untersuchungen ergaben eine partielle Gewebsstrukturierung während des in-situ Tissue Engineering Prozesses des Konstruktes.

Schlussfolgerungen. Unsere Studie demonstrierte, dass das fetale Schafmodel für Tissue Engineering der Speiseröhre geeignet ist. Des Weiteren vermindert die insitu Technik den mechanischen Stress, den das Konstrukt während der Boluspassage bei Verwendung von direkter in-vivo Tissue Engineering Techniken ausgesetzt ist.

\section{4}

\section{Simulation of acute coronary syndrome in the rat heart}

M. Kreibich ${ }^{1}$, E. Dzilic ${ }^{1}$, M. Hasun ${ }^{1}$, A. Baumgartner ${ }^{1}$, D. Santer ${ }^{1}$, P. Moser ${ }^{1}$, J. Kremer ${ }^{1}$, F. Nagel ${ }^{1}$, B. K. Podesser ${ }^{1,2}$, K. Trescher ${ }^{1,2}$

${ }^{1}$ Ludwig Boltzmann Cluster for Cardiovascular Research, Vienna, Austria; ${ }^{2}$ LK St. Poelten, Department of Cardiac Surgery, St. Poelten, Austria

Background. Mortality for emergency bypass surgery after Acute Coronary Syndrome accounts for up to $46.7 \%$. 
Aim of this study is to establish an acute Ischemia/Reperfusion-model in the rat to evaluate Ischemia/Reperfusion damage depending on a change in reperfusion time, and to improve intraoperative myocardial protection.

Methods. Following temporary LAD ligature $\left(60^{\prime}\right)$, male Sprague Dawley rats were randomly assigned to $60^{\prime}$ [group 1; $n=8$ ] or $120^{\prime}$ [group $2 ; n=9$ ] in-vivo reperfusion. Subsequently, hearts were excised and evaluation of hemodynamic parameters was performed on an erythrocyte perfused isolated working heart during $45^{\prime}$ baseline measurements, $60^{\prime}$ of Custodiol-protected ice cold ischemia and $45^{\prime}$ of postischemic reperfusion.

Results. In both groups, global ex-vivo ischemia significantly reduced postischemic external heart work (group 1: $70 \pm 20 \%$; group 2: $82 \pm 13 \%, p<0.01$ ) and cardiac output (group 1: $74 \pm 17 \%$; group 2: $83 \pm 11 \%$, $p<0.01)$ compared to preischemic baseline. Coronary flow (CF) was significantly reduced only after $2 \mathrm{~h}$ of reperfusion (group 1: $102 \pm 16 \%$, n.s.; group 2: $85 \pm 16 \%$, $p<0.05)$. Between the groups there was a significant difference in the recovery of CF with better recovery after $1 \mathrm{~h}$ reperfusion $(p<0.05)$.

Conclusions. We were able to establish a standardized, reproducible in-vivo rat Ischemia/Reperfusionmodel. In this model, protective effects of different cardioplegic solutions can be evaluated.

\section{5}

\section{A non suture cuff technique facilitates hemiface transplantation in mice - dawn of a new CTA animal model}

B. Kern ${ }^{1}$, R. Sucher ${ }^{1,2}$, C. Lin ${ }^{1}$, B. Zelger ${ }^{3}$, J. Pratschke ${ }^{2}$, S. Schneeberger ${ }^{1,2}$, W. Lee ${ }^{1}$, G. Brandacher ${ }^{1,2}$

${ }^{1}$ Department of Plastic and Reconstructive Surgery, Johns Hopkins University School of Medicine, Baltimore, USA; ${ }^{2}$ Department of Visceral, Transplant and Thoracic Surgery, D. Swarovski Research Laboratory, Innsbruck Medical University, Innsbruck, Austria; ${ }^{3}$ Department of Pathology, Innsbruck Medical University, Innsbruck, Austria

Background. To date rat-models of experimental-facetransplantation have been widely used. Since the mouse, however, would be a superior model for composite-tissueallotransplantation (CTA) research, we developed a novel surgical technique for allograft revascularization allowing us to perform hemiface-transplantation in mice.

Methods. BALB/c hemifacial flaps were transplanted to BALB/c (group1) or C57BL6 (group 2) recipients using a non-suture-cuff-technique for vascular anastomosis $(n=6)$. Donor operation consisted of harvesting a myocutaneus hemiface flap with intact common-carotid-artery (CCA) and external-jugular-vein (EJV) using superfine microsurgical instruments. In the recipient, the graft was transplanted in an orthotopic position using the CCA and EJV for revascularization.
Results. Transplants could be performed with a high success rate $(78 \%)$. Operation time was comparable in all groups and lasted $120 \pm 15 \mathrm{~min}$ for the donor and 150 $\pm 12 \mathrm{~min}$ for the recipient. All syngeneic grafts survived long-term ( $>100$ days). Allograft rejection in group 2 occurred within 14 days. H\&E stains of syngeneic grafts revealed unaltered muscle and skin histology. Allogenetic grafts gradually showed distinct BANFF rejection patterns over time of rejection similar to those observed after human-face-transplantation.

Conclusions. This is the first model illustrating that mouse hemiface allotransplantation is technically achievable. The microsurgically demanding procedure may be used to investigate basic-immunology and rejection as well as address questions related to nerve-regeneration in reconstructive-face-transplantation.

\section{6}

\section{Oxygen and carbon dioxide measuring principles for organ failure in extracorporeal circulation during cardiac surgery}

\author{
P. Curcic ${ }^{1}$, I. Knez', I. Ovcina1, E. Beran', V. Ribitsch², \\ M. Cajlakovic ${ }^{2}$, J. Krumnikl ${ }^{3}$, K. Tscheliessnigg ${ }^{1}$ \\ ${ }^{1}$ Klinische Abteilung für Herzchirurgie, Universitätsklinik

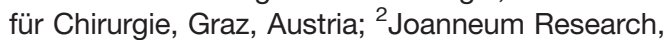 \\ Graz, Austria; ${ }^{3}$ Klinische Abteilung für Herz-, Gefäss- \\ und Thoraxanästhesie, Graz, Austria
}

Background. In an experimental animal model, we simultaneously evaluated metabolic variables and organ specific parenchymal $\mathrm{pO}_{2} / \mathrm{pCO}_{2}$ exchanges detected from parieto-temporal lobe of cerebrum, left ventricular myocardium and right hepatic lobe.

Methods. The presented $\mathrm{O}_{2}$ method is based on the phosphorescent dye Pt tetra-(pentafluorophenyl)porphine (PtTFPP), which is homogeneously dissolved in a polystyrene matrix and coated onto the tip of an optical fiber. A solid type of $\mathrm{CO}_{2}$ fiber optical sensor using an ionpair technique to encapsulate lipophilic $\mathrm{pH}$ indicator into hydrophobic polymer and employing RET as sensing scheme was used to measure phase modulation fluorometry. Pigs $(30.7 \pm 2.5 \mathrm{~kg})$ were randomized to group A (conventional cardiopulmonary bypass; $\mathrm{CPB}$ ) and to group B (mini-extracorporeal circulation, mini-EEC), the perfusion systems were minimized and adjusted to basic variables comparable to congenital extracorporeal perfusion settings.After baseline measurements $\mathrm{CPB}$ was induced, the aorta was crossclamped for $60 \mathrm{~min}$, the test animals were reperfused for $30 \mathrm{~min}$ and reobserved for another 30 min off-bypass.

Results. ANOVA emphasized significantly higher cerebral $\mathrm{pO}_{2}$ levels $(p=0.007)$ in group $\mathrm{B}$, while $\mathrm{pCO}_{2}$ levels appeared similar.In contrast, both hepatic and myocardial $\mathrm{pCO}_{2}$ levels were significantly higher in group A $(p=0.004)$, while $\mathrm{pO}_{2}$ levels reacted homogenously. 
Conclusions. Under standardized conditions, miniEEC produced less lactate and showed significantly favourable results concerning $\mathrm{O}_{2} / \mathrm{CO}_{2}$ metabolism and transfusion demand.

\section{7}

The efficacy of fibrin sealant fixation of hydrophobic implants in experimental onlay repair in rats

\section{S. Gruber-Blum ${ }^{1}$, A. Petter-Puchner ${ }^{2}$, J. Brand ${ }^{1}$, H. Redl ${ }^{1}$, K. Glaser ${ }^{2}$, R. Fortelny ${ }^{2}$ \\ ${ }^{1}$ Ludwig Boltzmann Institute for Experimental and Clinical Traumatology, Vienna, Austria; ${ }^{2}$ Department of Visceral and Tumor Surgery, Wilhelminenspital der Stadt Wien, Vienna, Austria}

Background. Fibrin sealant (FS) mesh fixation has gained increasing popularity in open and laparoscopic hernia repair and works especially well with hydrophilic implant materials, e.g. titanized meshes. The aim of this study was the assessment of FS fixation of hydrophobic implants. A polypropylene mesh coated with $\alpha-\Omega$ fatty acid (CQ, c-qur lite ${ }^{\mathbb{R}}$, Atrium) was tested in experimental onlay repair. Preserved pores facilitate FS fixation.

Methods. Sixteen CQ $(2 \times 2 \mathrm{~cm})$ were implanted in 8 male Sprague Dawley rats. Two different groups were investigated. Meshes were either fixed with FS 4 I.U. only (treatment) or sutured only (control). Follow-up was four weeks. Evaluation criteria were mesh dislocation, seroma formation, tissue integration and adhesion formation. Histology was performed (Hematoxilin Eosin staining and Cytokeratin staining).

Results. Despite the hydrophobic mesh coating and excessive movements of the animals during the observation period, FS alone yielded a reliable fixation. No mesh dislocation occurred, no seroma was detected and mesh integration was excellent.

Conclusions. Fixation of hydrophopic meshes with FS in onlay repair is feasible.

\section{8}

\section{Therapie peripherer Nervenläsionen} mittels einer adaptiven Nervenprothese: prospektiv randomisierte Studie an Minipigs

G. Tomasch ${ }^{1}$, D. Nagele-Moser ${ }^{1}$, D. Rafolt ${ }^{2}$, J. Waldert ${ }^{3}$, A. Berghold ${ }^{4}$, S. Uranüs ${ }^{1}$

${ }^{1}$ Sektion für Chirurgische Forschung, Graz, Austria;

${ }^{2}$ Zentrum für Med Physik und Biomed Technik, Wien, Austria; ${ }^{3}$ Landesnervenklinik Sigmund Freud, Graz, Austria; ${ }^{4}$ Institut für Med Informatik, Statistik u. Dokumentation, Graz, Austria
Grundlagen. Bei der Behandlung einer peripheren traumatischen Nervendurchtrennung konnte bisher mit mikrochirurgischer Koaptation, die eine spezialisierte Technik darstellt und nur in Zentren mit entsprechender Infrastruktur möglich ist, eine zufriedenstellende funktionelle Regeneration erzielt werden. Ziel der Studie ist eine neue Rekonstruktionsmethode mittels einer künstlichen Prothese $\mathrm{zu}$ entwickeln, die ohne aufwendige Technik anwendbar ist und dieselbe Behandlungsqualität bringt.

Methodik. Es wird an 35 weiblichen Minipigs im Alter von ca.12 Monaten der Nervus Ischiadicus durchtrennt. Nach der Randomisierung werden 15 Tiere mit der neuartigen Nervenprothese behandelt, 15 Tiere mittels mikrochirurgischer Koaptationsnaht versorgt und 5 Tiere werden ohne adaptive Intervention nach der Durchtrennung als Kontrollgruppe beobachtet. Während des 10monatigen Nachbeobachtungszeitraumes werden in 8-wöchigen Abständen elektrophysiologisch die Axonaussprossung und sonographisch die Muskelkonditionierung evaluiert. Am Studienende wird eine histopathologische Untersuchung durchgeführt.

Ergebnisse. Nach den vorliegenden Ergebnissen kann eine der Koaptation gleichwertige Nervenregeneration erreicht werden. Die beträchtliche Zeitersparnis beim Einbau der Prothese ist ein weiterer Gewinn dieser neuen Rekonstruktionsmethode.

Schlussfolgerungen. Bisherige Resultate ermutigen, dass die Implantation dieser Nervenprothese eine gute und effektive Regeneration herbeiführen und somit eine neue und einfach einsetzbare therapeutische Option zur peripheren Nervenläsionsversorgung darstellen könnte.

\section{Hauptsitzung - Komplikationsmanagement 1: Herdsanierung}

\section{9}

\section{A new treatment of the "open abdomen" with controlled negative pressure} and a new device*

\section{S. Reich-Weinberger ${ }^{1}$, M. Schmitz ${ }^{2}$, D. Öfner ${ }^{1}$ \\ ${ }^{1}$ University Hospital for Surgery of the PMU, Salzburg, Austria; ${ }^{2}$ Lohmann \& Rauscher, Rengsdorf, Germany}

Background. Although negative pressure therapy led to advancements in case of "open abdomen", modalities of treatment are barely standardized.

With this open case study, we would like to address this issue and offer a clinical procedure using NPWT and a new device*

Methods. This study comprises of 22 patients that suffered from advanced peritonitis/abdominal compart- 
ment syndrome. The concept of "damage control" was performed.

In order to control exudates and to reduce edema, the new film* was applied directly onto the omentum; fillingup with PU-foam, occlusion with PU-film and connection to negative-pressure ${ }^{* *}$ followed.

Results. This new treatment led to impressive results - optimal exudate management

- microbial loaded exudates were directly transported through the inner side of the film* minimizing the risk of infection spread

- primarily closure of all abdominal walls and reduction of secondary healing

- reduction of mortality down to $13.6 \%$.

Conclusions. The implementation of the new doublelayered-film* into the procedure of damage control improves the convalescence markedly: The unique properties enable fast closure of the abdominal wall; reduce the rate of secondary healing and mortality. Further, the use of this new film* offers great chances of cost benefits in the daily challenge.

\footnotetext{
* Suprasorb CNP Drainage Film. ** Suprasorb CNP.
}

\section{0}

\section{Einsatz von Abdominal VAC bei perforierenden Darmverletzungen}

\section{F. M. Birkfellner, H. Nehoda, B. Lade}

\section{a.ö. BKH St. Johann i.T, St.Johann i.T., Austria}

Grundlagen. Für Patienten stellen perforierende Darmverletzungen mit bereits fortgeschrittener generalisierter Peritonitis (Hinchey III\&IV) eine lebensbedrohliche Situation dar und bedürfen einer Notfalloperation. Der neu entwickelte Behandlungsalgorithmus mit Einlage eines Abdominal VAC ${ }^{\circledR}$ Systems stellt eine gute Möglichkeit dar, um die Darmkontinuität ehest möglich wiederherzustellen und eine Stomaanlage zu vermeiden.

Methodik. Der genannte Algorithmus wurde an unserer Abteilung für drei verschiedene chirurgische Vorgangsweisen angewandt. Gruppe 1: Laparatomie mit Darmteilresektion, Blindverschluß, Lavage, VAC-Anlage und Second-Look-Operation mit Reanastomosierung. Gruppe 2: Primäre Defektübernähung, Lavage, VAC-Anlage und Second-Look-Operation. Gruppe 3: Primäre Anastomosierung mit Lavage, VAC-Anlage und Second-LookOperation.

Ergebnisse. Notfalllaparatomien war bei allen 14 2009/10 behandelten Patienten (median 72a, acht Männer, sechs Frauen) mit perforierendem Ereignis mit Peritonitis (Hinchey III\&IV) indiziert. Neun Patienten erlitten eine Sigmaperforation, zwei Patienten eine Dünndarmperforation, drei einen perforierenden Dünndarmileus.
In Gruppe $1(n=10)$ wurden keine Komplikationen beobachtet, während in Gruppe $2(n=2)$ ein Patient aufgrund der schlechten Gerinnung, in Gruppe $3(n=2)$ ein Patient aufgrund einer Anastomosendehiszenz verstarb. Nach medianem Verweilen von fünf Tagen an der ICU und einer medianen Hospitalisierung von 24 Tagen verließen $85 \%(n=12)$ der Patienten anastomosiert und in gutem Allgemeinzustand unsere Abteilung.

Schlussfolgerungen. VAC-Anlage und mit geplanter Second-Look-Operation stellt eine gute Alternative bei der Behandlung von perforierenden Darmereignissen mit 4-Quadranten-Peritonitis (Hinchey III\&IV) hinsichtlich Sepsis-Management und Anastomosenanlage dar.

\section{3}

\section{Frühinfekt nach Inlaywechsel einer Hüft TEP - VAC Instill führte zum Erfolg}

\author{
S. E. Gabor, T. Niernberger, F. Stangl, \\ C. Kühbacher, H. Rabl
}

Abteilung für Chirurgie, Leoben, Austria

Fallbericht eines 67 jährigen Patienten mit einem Inlaywechsel (Inlay und Kopfwechsel) bei InlayVersagen einer TEP der linken Hüfte (kombiniert AHS-Depuy). Der Eingriff wurde im März 2010 durchgeführt. Der Eingriff sowie der postoperative Heilungsprozeß verlaufen komplikationslos so das der Patient am 10. postoperativen Tag mit blanden Wundverhältnissen in häusliche Pflege entlassen wurde. Am 15. postoperativen Tag erfolgte die Wiederaufnahme wegen eines infizierten Seroms zur Antibiotikatherapie sowie operativen Revision. Es erfolgten multiple Eingriffe mit Nekrektomie, Lavage sowie Anlage eines konvention.VAC. Im Rahmen der Eingriffe wurden zahlreiche Abstriche durchgeführt. Dabei zeigt sich eine Infektion mit E. coli sowie Enterobacter cloacae. Es gelang schließlichder sekundäre Wundverschluß und der Patient wurde nach 6 Wochen nach Hause entlassen. Nach nur einer Woche erfolgte jedoch die neuerliche Wiederaufnahme mit Fieber und Infektzeichen an der Wunde. In weiterer Folge entschieden wir uns nun zur Anlage eines VAC Instill. Im Rahmen des Eingriffs wurde das Instill System sowohl ventral als auch dorsal des Femurs platziert um eine kontinuierliche Lavage des gesamten Wundbettes zu erreichen.

In Summe waren 3 Eingriffe notwendig bis eine Sekundärnaht möglich war und der Patient konnte schließlich mit blandem Wundverhältnissen entlassen werden. Seit seiner Entlassung im Juni ist kein Reinfekt aufgetreten. 


\section{4}

\section{Reinfection after Sternal Repair for Deep Sternal Wound Infection}

\section{S. Raab, M. Beyer, L. Lampl}

Klinikum Augsburg, Cardiothoracic Surgery, Augsburg, Germany

Among 52 sternal osteosyntheses in deep sternal wound infections we observed 12 reinfections after plate or bridging osteosynthesis (Synthes ${ }^{\circledR}$ or Stratos ${ }^{\circledR}$ ) from 2006 to 2010. In case of a reinfection our policy has been to keep in place osteosynthetic material up to proper consolidation of the sternal bone. This is in contrast to the usual procedure with infected osteosynthetic material where a complete removement should always be achieved. As a first step in our protocol a full length reopening of the skin has been performed. Consecutively we installed a VAC $^{\circledR}$-System (KCI). Two to four weeks afterwards, we either closed the wound secondarily or covered the osteosynthetic material with omentum (2 cases) or rectus abdominis muscle flap. In five cases we removed osteosynthetic material after sternal consolidation. In all cases with soft tissue coverage we could keep them in place as well as in three cases with direct closure of the sternal wound. Only one patient died due to mediastinitis causing intractable infection. All other patients survived.

\section{5}

Die Faszienverschlussrate als wesentlicher Qualitätsparameter in der Behandlung des offenen Abdomens mit sekundärer Peritonitis

\section{S. Stättner, T. Hoblaj, S. Thalhammer, J. Karner}

SMZ Süd, Kaiser Franz Josef Spital, Abteilung Chirurgie, Wien, Austria

Grundlagen. Die Behandlung des schwergradig septischen Abdomens stellt eine große Herausforderung an das Behandlungsteam dar. Unter Zuhilfenahme der V.A. C. Therapie konnte eine Verbesserung im Überleben erzielt werden. Die hohe Rate an Hernien stellt jedoch ein großes Problem dar. Im SMZ Süd setzen wir seit 2 Jahren „dynamische Nähte“ in Kombination mit der VAC Therapie ein.

Methodik. Prospektive Datenerhebung von 15 Patienten, die mit der neuen Technik und VAC Therapie bei abdomineller Sepsis behandelt wurden. Ein Vergleich mit aktueller Literatur und historischen Daten aus dem eigenen Krankengut wird erstellt.

Ergebnisse. 15 Patienten wurden großteils von 1 Chirurgen therapiert, die Verbandswechsel fanden 48 stündlich statt. Die perioperative Mortalität betrug $15 \%$, die Gesamtmortalität mit 6 Monaten Nachbeobachtung $38 \%$.
Ein direkter Faszienverschluss konnte nach „intention to treat" bei $87 \%$ der Patienten und nach „per protocoll“ Analyse bei $92 \%$ der Patienten erzielt werden. Die Fistelrate betrug $13 \%$, 1 Patient musste wegen Retention CT gezielt drainiert und 2 Patienten wegen Blutung revidiert werden. Spalthautdeckung war in keinem Fall notwendig.

Schlussfolgerungen. Die Anwendung dynamischer Nähte in Kombination mit der V.A.C. Unterdrucktherapie ist eine effektive und sichere Methode beim septischen offenen Abdomen. Langzeitanalysen bezüglich ventraler Hernien stehen aus, eine Spezialisierung und abteilungsinterne Standards sind in jedem Fall sinnvoll.

\section{6}

\section{Die Rolle der Plastischen Chirurgie bei komplizierten Bauchwanddefekten}

\section{J. Roka-Palkovits, O. C. Aszmann, W. Happak, W. Haslik, L. Kamolz, B. Karle, H. B. Kitzinger, H. Kubiena, T. Rath, K. F. Schrögendorfer, M. Frey}

Plastische und Rekonstruktive Chirurgie/Medizinische Universität Wien, Wien, Austria

Bei aus komplizierten Verläufen im Rahmen der Abdominalchirurgie resultierenden Bauchwanddefekten existieren im Wesentlichen zwei Probleme, die die Ausschöpfung plastisch chirurgischer Techniken benötigen: erstens der große Bauchwanddefekt mit muskulärem Funktionsverlust und zweitens der komplette Bauchwanddefekt einschließlich des Peritoneums und der dermalen Decke, mitunter kompliziert durch begleitende, intestinale Stomata.

An unserer Abteilung wurden von Jänner 2002 bis Jänner 2011 insgesamt 86 Patienten mit Defekten der Bauchwand behandelt. Darunter fanden sich 6 Patienten mit exzessiv großen Narbenhernien und funktionell insuffizienter Bauchdecke und 8 Patienten mit einem ausgedehnten, kompletten Defekt der Bauchdecke.

Anhand instruktiver Beispiele werden die derzeitigen Möglichkeiten mit besonderer Beachtung spezieller plastisch chirurgischer Techniken entsprechend der lokalen Ausgangslage und des Allgemeinzustandes des Patienten vorgestellt.

\section{7}

Chirurgischer Zugang zur Nekrosektomie nach interventionell-radiologischer Drainage bei nekrotisierender Pankreatitis - Erfahrungen aus dem eigenen Krankengut

T. Hoblaj ${ }^{1}$, S. Stättner ${ }^{1}$, F. Karnel ${ }^{2}$, G. Strau ${ }^{2}$, W. Hackl ${ }^{3}$, J. Karner ${ }^{1}$ 
${ }^{1}$ SMZ Süd KFJ, Chirurgische Abteilung, Wien, Austria; ${ }^{2} \mathrm{SMZ}$ Süd KFJ, Zentralröntgeninstitut, Wien, Austria; ${ }^{3} \mathrm{SMZ}$ Süd KFJ, Abteilung für Anästhesie und operative Intensivmedizin, Wien, Austria

Grundlagen. Die Indikation zur chirurgischen Intervention bei nekrotisierender Pankreatitis ist nicht klar definiert, es besteht aber ein Konsens zur chirurgischen Nekrosektomie bei Vorliegen von infizierten Nekrosen und bei protrahiertem Mehrorganversagen im Verlauf einer konservativen Behandlung. Insbesondere nach andauernder Etappenlavage oder offener Wundbehandlung mit Vakuumsystem stellen Rest- oder Folgezustände im Sinne von infizierten peripankreatischen Nekrosen, Retentionen oder Abszessen allerdings eine interdisziplinäre Herausforderung dar.

Methodik. Anhand von Fallbeispielen aus dem eigenen Krankengut werden verschiedene Behandlungsstrateigien dargestellt.

Fall 1: Rezidivierende, infizierte, peripankreatische Retentionen unter offener Abdominalbehandlung mit Etappenlavage, Vakuumtherapie unter sukzessiver intestinaler Blockbildung. Chirurgisches Debridement über einen retroperitonealen Zugang in der linken Flanke entlang einer CT gezielt gesetzten Drainage als "guide wire“.

Fall 2: Totalnekrose des Pankreas mit Superinfektion unter offener Abdominalbehandlung mit Etappenlavage, Vakuumtherapie und interventionell-radiologischer Drainage. Nekrosektomie durch transabdominellen Zugang.

Schlussfolgerungen. Neben der Frage des Zeitpunktes einer chirurgischen Intervention bei nekrotisierender Pankreatitis stellt vor allem auch die Strategie des chirurgischen Zugangs zu Nekrosearealen, Retentionen oder Abszessen eine Herausforderung dar. Interventionell-radiologisch platzierte Drainagen können neben der Ableitung von infizierten Flüssigkeitsansammlungen auch eine wertvolle Orientierungshilfe für den chirurgischen Zugang darstellen.

\section{8}

\section{Behandlungsschema von PatientInnen mit nekrotisierender Fasziitis}

\section{G. Weigel, J. Schratt, M. Schintler, S. Spendel}

Uinversitätsklinik für Chirugie, Klinische Abt. Plastische, Ästhetische und Rekonstruktive Chirurgie, Graz, Austria

Grundlagen. Die nekrotisierende Fasziitis ist eine lebensbedrohliche, rasch fortschreitende Infektion des Subkutan- und Fasziengewebes. Da es weder eindeutige Laborwerte, noch klinische Zeichen gibt, welche auf das Bestehen einer nekrotisierenden Fasziitis hinweisen, ist es schwierig gleich zu Beginn die richtige Diagnose zu stellen.

Methodik. Es handelt sich um eine retrospektive Studie (1999-2009), welche am Universitätsklinikum Graz durchgeführt wurde. Untersucht wurden die Daten von 60 PatientInnen mit der Diagnose nekrotisierender Fas- ziitis. Evaluation von: Alter, Geschlecht, Komorbiditäten, Lokalisation, Mikrobiologie, Laborwerten, Dauer des stationären Aufenthaltes, Therapie.

Ergebnisse. 45 Männer und 15 Frauen waren betroffen, mittleres Alter aller PatientInnen 60 Jahre. Die Mortalität lag bei $22 \%$. Die Extremitäten waren in 28 Fällen betroffen. Häufigste Komorbiditäten: kardiale Erkrankungen ( $57 \%)$ und Diabetes mellitus (55\%). In 58 Fällen war mehr als eine Komorbidität nachzuweisen. Am häufigsten kam es zu einer polymikrobiellen Infektion. Bezüglich der Therapie (radikale Débridements, Antibiotika, HBO-Therapie, Unterdruck-Therapie, Amputationen) konnte kein statistisch signifikanter Unterschied zwischen überlebenden und verstorbenen PatientInnen gefunden werden.

Schlussfolgerungen. Essentiell für das Überleben der PatientInnen ist die frühest mögliche Durchführung einer chirurgischen Sanierung und die Verabreichung von Breitbandantibiotika, da nur so das Fortschreiten des infektiöses Prozesses verhindert werden kann. Adjuvant ist der Einsatz einer HBO-Therapie möglich.

\section{Österreichische Gesellschaft für Thorax- und Herzchirurgie: Herzchirurgie - Congenital und Varia}

\section{9}

\section{Minimally invasive repair of atrial septal defects - extending surgical complexity in daily routine}

\section{K. Stifter, J. Kilo, A. Heinz, H. B. Hangler, M. Grimm, L. Müller}

Medical University of Innsbruck, Innsbruck, Austria

Background. Atrial Septal Defects (ASD) apply for 5$10 \%$ of congenital heart disease. The extent of the defect rises from a persistent foramen ovale to the sinus venosus defect and anomalous drainage of one or more pulmonary veins (PAPVC). We reviewed our experience on the minimally invasive surgical technique.

Methods. We reviewed all patients undergoing minimally invasive ASD-closure at our institution from 2001 to 2010. Analysis was performed concerning ASD-pathology, patient characteristics and operative variables.

Results. From 01/2001 through 12/2010, 38 patients underwent minimally invasive ASD-closure. 20 defects were closed directly, 18 by patch. Since 2007, also Sinus Venosus Defects are successfully treated minimally invasive $(n=10)$. Intraatrial baffle correction of PAPVC and reconstruction of vena cava superior is feasible $(n=4)$. The mean age was 40.7 years, mean weight was $70.2 \mathrm{~kg}$. Mean aortic crossclamp time was $53.7 \mathrm{~min}$. There was no fatality and no severe perioperative complications. One 
patient experienced occlusion of femoral artery late postoperatively $(2.6 \%)$.

Conclusions. Minimally invasive correction of defects of the intraatrial septum has successfully been introduced into clinical routine at our institution. Operative morbidity is very low and even complex reconstructions can be performed with good results. Median sternotomy is only performed in smaller children any longer at our institution.

\section{0}

\section{Everolimus in paediatric and infant heart transplantation}

\section{Schweiger, H. Lehmkuhl, S. Schubert, N. Hiemann,} M. Dandel, C. Knosalla, O. Grauhan, R. Hetzer

Deutsches Herzzentrum Berlin, Berlin, Germany

Background. So far no experience exists on the use of Everolimus (EvA) as de-novo therapy in paediatric cardiac transplantation and infants.

Methods. Figty-two paediatric heart transplant recipients receiving EvA (Group A, n: 24) or mycophenolat mofetil (Group B, n: 28) were investigated. The study variables were: efficacy of EvA, survival, safety, CsA trough levels, biopsy proven acute rejection (BPAR), laboratory values and angiography evidence of cardiac allograft vasculopathy.

Results. Six children died (Group A n: 1, Group B n: 5, $p=0.200$ ), one child had to discontinue EvA (renal failure). Mean EvA trough levels after one week were 5.08 $\pm 2.42 \mathrm{ng} / \mathrm{ml}$ and $4.8 \pm 1.3 \mathrm{ng} / \mathrm{ml}$ after 24 months. CsA trough levels in Group A were $167.1 \mathrm{ng} / \mathrm{ml} v s .283 .8 \mathrm{ng} / \mathrm{ml}$ (6 month), $134.1 \pm 50.7 \mathrm{ng} / \mathrm{ml}$ vs. $234.9 \pm 23.8 \mathrm{ng} / \mathrm{ml}(12$ month) and $79.3 \mathrm{ng} / \mathrm{ml} v s .76 .0 \mathrm{ng} / \mathrm{ml}$ (24 month). Two children died due to acute cellular rejection (Group B). Two cases out of each group experienced BPAR grad 2R $(p=0.043)$. Creatinine levels increased in both groups in the first three weeks $(1.42 \mathrm{mg} / \mathrm{dl} v s .0 .48 \mathrm{mg} / \mathrm{dl})$ returning to normal values $(1.1 \mathrm{mg} / \mathrm{dl} v s .0 .99 \mathrm{mg} / \mathrm{dl})$.

Conclusions. EvA with reduced CsA trough level regime in a paedriatic population was safe, effective and comparable to other IS regimes.

\section{1}

Erfahrungen mit Korrektur der isolierten totalen Lungenvenenfehlmündung ohne Kreislaufstillstand

\section{E. Sames-Dolzer, R. Mair, M. Innerhuber, G. Tulzer, C. Gross}

Kinderherz-Zentrum Linz, Linz, Austria

Grundlagen. Die Korrektur der isolierten totalen Lungenvenenfehlmündung erfolgte klassischerweise in Kreis- laufstillstand bei tiefer Hypothermie. An unserem Zentrum wurde die Operation bei kontinuierlichem Bypass und milder Hypothermie durchgeführt. Operationsergebnisse und Langzeitverlauf dieses seltenen lebensbedrohlichen Vitiums sollen untersucht werden.

Methodik. Seit 1997 wurden am Kinderherzzentrum Linz 28 Kinder mit totaler Lungenvenenfehlmündung korrigierend operiert (KG zwischen $1,2 \mathrm{~kg}$ und $8,1 \mathrm{~kg}$, mean $3,35 \pm 1,2 \mathrm{~kg}) .8$ von 28 Kindern zeigten präoperativ eine lebensbedrohliche Lungenvenenobstruktion. Alle wurden dringlich am kontinuierlichen Bypass durch Anastomosierung d.Lungenvenenkonfluens an den linken Vorhof operiert.

Ergebnisse. Die Operation konnte in allen Fällen am kontinuierlichen Bypass durchgeführt werden. Die frühe postoperative Mortalität lag bei 1/28 Kindern. 2 frühgeborene Patienten mit höchstem Risikoprofil verloren wir nach protrahierter Intensivtherapie 2,5 bzw 4 Monate postoperativ an kardiorespiratorischem Versagen bei persistierender pulmonaler Hypertonie. 1 Patientin verstarb an einer Pneumonie 3 Monate nach Entlassung. 24 Kinder zeigten einen unauffälligen Langzeitverlauf ohne Restenose oder Reoperation. Alle 4 verstorbenen Patienten litten an einer obstruktiven Form der Lungenvenenfehlmündung.

Schlussfolgerungen. Die lebensrettende Korrekturoperation ist am kontinuierlichen Bypass mit geringer Mortalität möglich. Die primäre Obstruktion des Lungenvenenabflusses zeigte vor allem bei Vorliegen von weiteren Risikofaktoren mit einer Mortalität von $50 \%$ eine schlechte Prognose. Der Langzeitverlauf ist nach komplikationsfreier Korrektur ausgezeichnet.

\section{2}

\section{Nekrotisierende Enterkolitis - eine diagnostisch und therapeutisch anspruchsvolle perioperative Komplikation der Korrektur angeborener Herzfehler}

\author{
S. Kargl ${ }^{1}$, R. Mair ${ }^{2}$, C. Prandstetter ${ }^{3}$, W. Pumberger ${ }^{1}$ \\ ${ }^{1}$ Abteilung für Kinder und Jugendchirurgie, Landes - \\ Frauen- und Kinderklinik LINZ, Linz, Austria; ${ }^{2}$ Abteilung \\ für Herz-,Thorax- und Gefäßchirurgie AKH, Linz, Austria; \\ ${ }^{3}$ Abteilung für Kinderkardiologie, Landes - Frauen- \\ und Kinderklinik Linz, Linz, Austria
}

Grundlagen. Kinder mit angeborenen Herzfehlern haben ein erhöhtes Risiko für die Entwicklung einer nekrotisierenden Enterokolitis. Die postoperative Phase ist problematisch, da hier Diagnosestellung und Therapiebeginn oft verzögert erfolgen.

Methodik. Die Patientenakten der Kinder mit operativer Vitienkorrektur der letzten 3 Jahren wurden begutachtet und diejenigen mit intraoperativ nachgewiesener NEC aufgearbeitet und verglichen.

Ergebnisse. Bei fünf Patienten konnte eine NEC diagnostiziert werden. Alle zeigten komplizierte Verläufe. 
Zwei Patienten verstarben als Folge der ausgedehnten Darmschädigung.

Schlussfolgerungen. Die nekrotisierende Enterokolitis (NEC) ist eine Erkrankung die vor allem Frühgeborene betrifft. Als durch verschiedene Faktoren ausgelöster Endpunkt eines intestinalen Gewebeschadens, wird der Krankheitsverlauf von der Reaktion des betroffenen Organismus bestimmt. Pathogenetisch spielen Ischämie, Mikroorganismen und enterale Ernährung eine Rolle.

Reifgeborene mit kongenitalen Herzfehlern haben im Vergleich mit herzgesunden Reifgeborenen ein erhöhtes Risiko an einer NEC zu erkranken. Interessanterweise scheint eine NEC bei Kindern mit kongenitalen Herzfehlern mit einer geringeren NEC-assoziierten Morbidität und Mortalität einher zu gehen und wird daher von manchen Autoren als eigene Entität gesehen. Im Gegensatz dazu ist die NEC als Komplikation der operativen Vitienkorrektur ein gefürchtetes, weil mit hoher Letalität behaftetes Ereignis. Der Herz - Lungen - Bypass begünstigt eine Ischämie im Splanchnicusgebiet und eine veränderte Darmpermeabilität, welche die fatale Keiminvasion erlaubt.

\section{3}

\section{ECMO - Outcome bei herzkranken Kindern}

\section{Innerhuber, R. Mair, E. Sames-Dolzer, E. Feichtinger, G. Tulzer, C. Cross}

Abteilung für Herz-, Thorax- und Gefäßchirurgie AKh Linz, Linz, Austria

Grundlagen. Die extrakorporale Membranoxygenierung stellt eine etablierte Methode zum vorübergehenden Herz- und Lungenersatz dar. Die Erfahrung bei neugeborenen Herzkindern mit niedrigem Geburtsgewicht sowie bei Single-Ventricle-Physiologie ist gering. Es soll gezeigt werden, dass die ECMO auch in diesen Fällen eine Therapieoption darstellt.

Methodik. Seit 2007 wurden am Kinderherzzentrum Linz 21 Kinder mit vorangegangener Herzoperation an die ECMO genommen. Das Lebensalter bei ECMO-Einbau betrug im Median $29\{14 ; 57\}$ Tage (7-130), das Gewicht im Median 3700 \{3080;4200\} g (2400-6820). Es handelte sich um 15 uni- und 6 biventrikuläre Herzen, in 16 Fällen konnte die Ursache als cardial, in 6 Fällen als pulmonal beurteilt werden. Der ECMO-Einbau erfolgte bei 12 Kindern unter Reanimationsbedingungen, die Reanimationsdauer betrug 10-120 Minuten.

Ergebnisse. Nach einer ECMO-Dauer von im Median 7 \{5;12\} Tagen (2-15) konnten 15 Patienten erfolgreich geweant werden, davon starben 5 Kinder während des stationären Aufenthaltes und 1 Patient 2 Monate nach Entlassung. 2011 sind 6 uni- und 3 biventrikuläre Herzkinder am Leben. 7 Überlebende werden in der Entwicklung als altersentsprechend bis leicht entwicklungsverzögert beurteilt, 2 als nicht altersentsprechend.

Schlussfolgerungen. Die extrakorporale Membranoxygenierung ist auch bei Kindern mit Single-Ventricle-Pal- liation eine wertvolle Option. Alter, Gewicht und Diagnose haben keinen erkennbaren Einfluss auf das Outcome.

\section{4}

Minimally invasive double valve surgery can safely be combined with additional procedures

\section{J. Kilo, H. B. Hangler, A. Heinz, K. Stifter, M. Grimm, L. Müller}

Medical University of Innsbruck, Innsbruck, Austria

Background. Although minimally invasive cardiac surgery is well established, diffusion of the technique is limited. Contraindications are not only conditional on the disease but also on the surgeon's experience and attitude. To evaluate disease related contraindications we investigated our patients receiving minimally invasive double valve surgery with or without additional procedures.

Methods. Three hundred and Thirty-one patients undergoing minimally invasive mitral valve surgery between 2001 and 2010 were analyzed. Additional tricuspid annuloplasty (TVP) was indicated for severe tricuspid regurgitation or tricuspid annular dilatation $>40 \mathrm{~mm}$. Left atrial ablation (RF-maze) was performed using unipolar radiofrequency.

Results. After implementation of the minimally invasive valve program, TVP was added after 54 successful isolated MV-procedures. Seventy patients (25.3\%) had combined mitral and tricuspid surgery. TV surgery was always performed as ring annuloplasty. Seventeen double valve patients had additional RF-maze. Twelve patients underwent closure of the left atrial appendage (LAA), 13 patients underwent additional PFO closure. Mortality in the double valve group was $1.4 \%$ and $0.45 \%$ in the MV only group ( $p=\mathrm{ns})$.

Conclusions. Minimally invasive TVP can be added safely to MV surgery. Further procedures like RF-maze, LAA- or PFO-closure can also safely be performed. Neither mortality nor major complications related to the combined procedures were increased.

\section{5}

\section{Angiographic evaluation of robotically assisted coronary anastomosis using conventional and CT-angiography}

\author{
N. Bonaros ${ }^{1}$, F. Weidinger ${ }^{1}$, B. Hofauer ${ }^{1}$, F. Plank ${ }^{2}$, \\ G. Feuchtner ${ }^{2}$, G. Friedrich ${ }^{3}$, M. Grimm ${ }^{1}$, T. Schachner ${ }^{1}$ \\ ${ }^{1}$ Department of Cardiac Surgery, Innsbruck, Austria; \\ ${ }^{2}$ Department of Radiology, Innsbruck, Austria; ${ }^{3}$ Department \\ of Cardiology, Innsbruck, Austria
}


Background. The aim of the study was to investigate the short-term quality of robotically sutured anastomoses by means of graft and multidetector CT angiography.

Methods. Robotically assisted coronary surgery was performed in 276 using the daVinci telemanipulation system, $51 \%$ and $96 \%$ of which underwent postoperative conventional graft angiography and CT angiography respectively. The majority of the patients underwent single or double arrested heart TECAB $(207 / 276), 21$ patients had a BH TECAB, 15 patients received composite grafts. Median angiographic follow up was 3 months (0.25-72).

Results. There were 160/319 (50\%) robotically sutured anastomoses evaluated by conventional graft angiography. There were 3 anastomoses with nonsignificant angiographic stenosis, 2 anastomoses with relevant stenosis $>50 \%, 1$ anastomotic occlusion, and 1 anastomosis to an incorrect target vessel. CT angiography revealed 1 anastomotic stenosis, 2 grafts with a string phenomenon as a result of competitive flow, 3 graft occlusions and one incorrect grafting site. In $153 / 160(96 \%)$ anastomoses evaluated by invasive angiography a perfect result was detected. CT angiography revealed a perfect postoperative result in 298/305 evaluated anastomoses (98\%).

Conclusions. Robotically sutured anastomosis can be performed with satisfying angiographic results. CT angiography can be used as an alternative for postoperative evaluation of relevant anastomotic dysfunction.

\section{6}

\section{Ursachen und Konsequenzen einer Konversion von Totalendoskopischen koronaren Bypassoperationen}

\section{T. Schachner ${ }^{1}$, N. Bonaros ${ }^{1}$, D. Wiedemann ${ }^{1}$, E. Lehr ${ }^{2}$, F. Weidinger ${ }^{1}$, G. Feuchtner ${ }^{1}$, D. Zimrin², J. Bonatti ${ }^{2}$}

${ }^{1}$ Medizinische Universität Innsbruck, Innsbruck, Austria; ${ }^{2}$ University of Maryland, Baltimore, USA

Background. Totally endoscopic coronary bypass surgery (TECAB), using daVinci ${ }^{\mathrm{TM}}$ telemanipulator, became reproducible at dedicated centers.

Methods. We performed TECAB in 326 patients. 242 single, and 84 multivessel CABG.

Results. 46/326 (14\%) of the TECABs were converted to a minithoracotomy in $5(2 \%)$ and a sternotomy in 41 (12\%) cases. Lima injury, epicardial injury, balloon endoocclusion problems, and anastomotic problems caused conversions. Conversion rate was significantly less for single vessel TECAB than multivessel TECABs $(10 \%$ vs. $25 \%, p=0.001)$. Other factors associated with lower conversion rates: non-learning curve case $(7 \%$ vs. $21 \%$, $p<0.001)$, and transthoracic assistance $(11 \%$ vs. $22 \%$, $p=0.018)$. In multivariate analysis learning curve case was the only independent predictor of conversion $(p=0.005)$. Conversion translated into increased transfusion of PRBC in the OR ( $3 v s .0$ units, $p<0.001$ ), longer ventilation (14 vs. 8 hours, $p<0.001$ ) and prolonged ICUstay (45 vs. 20 hours, $p=0.001$ ). Hospital mortality was $0.6 \%$ with 1 patient in the conversion group $(2.2 \%)$ and 1 patient in the non-converted group $(0.4 \%, p=\mathrm{ns})$. There was no difference in freedom from angina or freedom from major adverse cardiac and cerebral events.

Conclusions. TECAB Conversion is primarily learning curve dependent and associated with increased morbidity. Long-term freedom from angina or MACCE does not seem to be influenced by conversion.

\section{7}

\section{Einsatz von Fucidin als neues Konzept in der perioperativen Prophylaxe in der Herzchirurgie: Reduktion der tiefen Sternuminfektionen}

\section{Fink, W. Wandschneider}

Klinikum Klagenfurt am Wörthersee, Abtlg. f. HerzThorax- und Gefäßchirurgie, Klagenfurt, Austria

Die tiefe Sternuminfektion nach kardiochirurgischen Eingriffen ist eine gefürchtete Komplikation, die eine signifikant erhöhte Morbidität und Mortalität nach sich zieht. Arrosionsblutungen führen nicht selten zum Tod des Patienten. Die Literatur gibt Infektionsraten zwischen $0,5 \%$ und $3 \%$ oder mehr an. In etwa $50 \%$ finden sich als Erreger koagulasenegative Staphylokokken gefolgt von Staphylokokkus aureus. Diese Erreger sind in der Antibiotikaprophylaxe zu berücksichtigen. An der Abteilung für Herz- Thorax- und Gefäßchirurgie werden jährlich im Durchschnitt 470 kardiochirurgische Elektiveingriffe durchgeführt. Da die beobachtete Rate an tiefen Sternuminfektionen mit 3,2\% nicht akzeptabel war, wurde ein neues Regime in der perioperativen Antibiotikaprophylaxe eingeführt. Die am häufigsten isolierten Keime waren oxacillinresistente koagulasenegative Staphylokokken. Hygienefehler als Ursache für die überhöhte Infektionsrate wurden ausgeschlossen. Fucidin ist ein altes, aber sehr potentes Staphylokokkenantibiotikum, das in seinem Spektrum neben Staphylokokkus aureus auch MRSA und koagulasenegative Staphylokokken abdeckt. In der Literatur ist bisher keine Beschreibung von Fucidin in der Prophylaxe bei kardiochirurgischen Eingriffen zu finden, einzelne Arbeiten zeigen jedoch eine gute Penetration ins mediastinale und myoperikardiale Gewebe, aber auch ins Sternum. Wir haben unsere perioperative Standardprophylaxe mit Cefuroxim durch Fucidin ergänzt. Durch diese Maßnahme wurde eine dramatische Reduktion der tiefen Sternuminfektionen von $3,2 \%$ auf $0,6 \%$ erreicht, das entspricht einer Reduktion von $80 \%$. 


\section{Österreichische Gesellschaft für Unfallchirurgie: Varia}

\section{8}

\section{Die posttraumatische Pyomyositis-Eine diagnostische und therapeutische Herausforderung}

\section{G. Schimpl' ${ }^{1}$, J. Bauer ${ }^{1}$, J. Schnöll' ${ }^{1}$, H. Nemec ${ }^{2}$ \\ ${ }^{1}$ Department of Paediatric Surgery, Salzburg, Austria; ${ }^{2}$ Paediatric Radiology, Salzburg, Austria}

Grundlagen. Die Pyomyositis (PM) ist eine bakterielle Infektion der Stammmuskulatur, die bei Jugendlichen nach sportlichen Aktivitäten auftritt.

Erreger sind in 50-90\% meist Methicillin resistente Staphylokokken.

Methodik. 4 Patienten im Alter von 12-17 Jahre wurden wegen Hüftschmerzen, Rückenschmerzen und einem rezidivierendem Abszess an der Thoraxwand zugewiesen. Diagnostisch wurden neben den Blutuntersuchungen, Ultraschalluntersuchungen (US), Computertomographien (CT), und Magnetresonanzuntersuchungen (MR) der betroffenen Region durchgeführt.

Ergebnisse. Bei 3 Patienten fand sich eine Leukozytose mit CRP und Interleukin-8 Erhöhung. Während der US nur unspezifische Weichteilveränderungen zeigte, fanden sich im CT und MR deutlich sichtbare entzündliche Veränderungen und $2 \times$ eine Abzessformation. 2 Patienten, einer mit einer PM im M. erector trunci und einer im M. obturatorius wurden konservativ behandelt. Bei 2 Patienten, einer mit einem Psoasabszess und einer mit einem Thoraxwandabszess wurde eine operative Abszessentleerung gemacht. Alle Patienten erhielten eine entsprechende antibiotische Therapie und damit konnte eine Ausheilung erreicht werden.

Schlussfolgerungen. Die PM ist selten und zur Abklärung ist die MR die Untersuchungsmethode der Wahl. Neben der antibiotischen Therapie ist bei abszedierenden Prozessen auch ein operatives Vorgehen indiziert.

\section{9}

APACHE II, ISS, NISS and prothrombin time as independent predictors of death in the polytrauma patient within the first 72 hours after admission: a multiple logistic regression analysis of 696 patients

\author{
L. Mica, K. Albrecht, M. Turina, G. A. Wanner, \\ C. Werner, H. Simmen
}

Trauma Surgery Zürich, Zürich, Switzerland
Polytrauma patients are at high risk to die due to acute physiological deterioration. The scoring systems ISS, NISS and APACHE II, and platelet-counts and prothrombin time at admission seem to be relevant risk-factors of patients early. The aim of this study was to evaluate independent predictors of early death at admission of the polytrauma patient.

A total of 696 patients (age $40.8 \pm 15.5$ years) with an ISS $\geq 17$ and were admitted to a level I trauma center were included into this retrospective study. The patients were subdivided into survivors and those who died within the first 72 hours. ISS, NISS and APACHE II score, and lactate, prothrombin time and $\mathrm{pH}$-value were collected. Data were compared with Mann-Whitney test and $\chi^{2}$-test. The significancy level was set at $p<0.05$. Predictive ability was evaluated by using ROC-curves. Independent predictors were analyzed by logistic regression analysis. Significantly increased ISS, NISS, APACHE II-scores and serum lactate, and significantly decreased values of prothrombin time and $\mathrm{pH}$ were found in the non-survivor group $(p<0.001)$. APACHE II, NISS, ISS score and the prothrombin time were found to be independent predictive values of death. Damage control-surgery and early resuscitation with blood-products could improve the survival of polytrauma patients.

\section{0}

\section{Repair of distal biceps tendon ruptures using suture anchors: technique, functional outcome and comparison with other fixation methods}

\section{Zosso, T. Hotz, M. Rudin, K. Käch \\ Kantonsspital Winterthur, Winterthur, Switzerland}

Background. Operative repair of distal biceps tendon ruptures is generally recommended. This study presents the results of surgical repair using suture anchors in a single-incision technique and compares them with the results of other fixation techniques.

Methods. Retrospective review of 31 patients (2 women, $29 \mathrm{men}$ ) treated for acute complete distal biceps tendon rupture between 1998 and 2010 using above-mentioned method. The patients were interviewed and underwent a clinical examination. They also completed three different rating systems for evaluation of the elbow (DASH, MEPI, Mayo Elbow Performance Index).

Results. Average age at injury time was 47 years (range: 32-68 years). The mean time between injury and surgery was 6 days (range: $0-27$ days). At a mean followup of 70 months (range: 10-152 months), patients presented satisfactory subjective and objective clinical results. Dynamometric tests showed satisfactory results both regarding maximum strength and endurance tests. One reoperation because of heterotopic ossification was 
done. One patient had to be reoperated for rerupture caused by a second accident. Neurologic complications or radioulnar synostosis didn't occur.

Conclusions. Repair of distal biceps tendon ruptures using bone anchors is safe and yields excellent clinical outcomes comparable with other repair techniques.

\section{1}

\section{Damage Control Surgery. Begriffsbestimmungen und Beispiele}

\author{
K. S. Wolff ${ }^{1}$, G. Pöschl ${ }^{2}$ \\ ${ }^{1}$ Heeresspital Wien, Chirurgische Abteilung, Wien, Austria \\ ${ }^{2}$ Heeresspital Wien, Anästhesiologische Abteilung, Wien, \\ Austria
}

Grundlagen. Damage Control Surgery (DCS) wurde zur Erhöhung der Überlebensfähigkeit Schwerverletzter am Gefechtsfeld als Sanitätsverfahren eingeführt.

Methodik. Analyse der NATO Vorschriftenlage.

Ergebnisse. CS wird als Sanitätsversorgungsprinzip im Einsatz unter zwei Bedingungen eingesetzt, die entweder jeweils allein oder in Kombination auftreten können: a) Verwundetenmassenanfall; Hier überschreitet die Anzahl der einsatzchirurgisch zu Versorgenden die Haltekapazität der aufnehmenden Sanitätseinrichtung. b) Die Verletzung ist so schwer, daß eine primäre Chirurgie die Kapazität (OP-Material, Intensivstation) der aufnehmenden Sanitätseinrichtung überfordert.

Wenn diese Bedingungen zutreffen, wird die Sanitätsversorgung auf DCS umgestellt. DCS bedeutet das Verschieben der wiederherstellenden Chirurgie auf einen späteren Zeitpunkt in einer höheren Versorgungsebene. DCS bedeutet Chirurgie gerichtet auf das Beheben lebensbedrohlicher Zustände, auf die Wiederherstellung des physiologischen Gleichgewichts durch chirurgischanästhesiologische Maßnahmen, insbesonders das Herstellen der Hämostase, Normalisierung des Flüssigkeitshaushaltes, Oxygenierung und Kontrolle der Eingeweideverletzungen.

Die Darstellung der DCS Prinzipien anhand eines Enterothorax im Einsatz als auch beim Verwundentenmassenanfall (MasCal) wird erfolgen.

Schlussfolgerungen. DCS ist ein militärisches Sanitätsversorgungsprinzip um bei materieller und/oder quantitativer Kapazitätsüberlastung die Anzahl Gefallener als Gefechtsfolge deutlich zu senken. Eine Anwendung im Zivilen erscheint daher nur im Massenanfall bei Katastrophen möglich. DCS als semantischer Begriff oder als chirurgisch-anästhesiologisches Verfahren ist daher im Krankenhausnormbetrieb unangebracht.

\section{2}

\section{Konservative Therapie der Milzruptur: Schockraumkonzept im Unfallkrankenhaus Linz}

\section{A. Kröpfl, M. Ponschab, M. Reischl, G. Steiner, S. Kapral} Unfallkrankenhaus, Linz, Austria

Grundlagen. Bei konservativer Behandlung von Milzverletzungen mit hämodynamischer Stabilität bleibt die sekundäre Milzruptur eine weiterhin auftretende, gefürchtete Komplikation.

Methodik. Die Einteilung der Milzverletzung erfolgt nach den Richtlinien der American Association for the Surgery of Trauma in die Grade I-V. In den Jahren 2007 bis 2010 kam es bei 25 primär konservativ behandelten Milzrupturen zum Auftreten von insgesamt 3 sekundären Laparatomien auf Grund akut auftretender hämodynamischer Instabilitäten, wobei dann immer eine Splenektomie erfolgte. Dies führte zu einer Inauguration eines speziellen Schockraumalgorythmus „Milz“ zur konservativen Behandlung der Milzverletzung bei hämodynamischer Stabilität unter Berücksichtigung des Rupturgrades der Milzverletzung als auch der Berücksichtigung der Gesamtverletzungsschwere des Patienten.

Ergebnisse und Schlussfolgerungen. Bei hämodynamischer Stabilität werden Milzverletzungen der Schweregrade I und II konservativ behandelt. Hämodynamisch stabile Milzrupturen Grad III werden bei einer Gesamtverletzungsschwere gemessen im ISS unter 25 Punkten konservativ behandelt., bei einem ISS von 25 und mehr Punkten erfolgt die Laparatomie. Hämodynamisch stabile Milzverletzungen der Schweregrade IV und V werden der Laparatomie zugeführt. Dabei wird das Ziel verfolgt, primär organerhaltend zu operieren. Ziel des neuen Algorythmus ist die möglichste Vermeidung von sekundären operativen Eingriffen bei plötzlicher hämodynamicher Instabilität nach primärer konservativer Behandlung von Milzrupturen, da diese Eingriffe sehr häufig zu Splenektomien führen.

\section{Hauptsitzung - Neue Techniken 2: Zugänge}

\section{3}

\section{Early clinical experience with per oral submucosal endoscopic myotomy} for the treatment of esophageal achalasia

E. Rieder ${ }^{1,2}$, C. M. Dunst ${ }^{3}$, L. L. Swanstrom ${ }^{2,3}$

${ }^{1}$ Department of Surgery, Medical University of Vienna, Vienna, Austria; ${ }^{2}$ Legacy Health, MIS Program, Portland, OR, USA; ${ }^{3}$ The Oregon Clinic, GMIS, Portland, OR, USA 
Background. Per oral submucosal endoscopic myotomy (POEM) has been demonstrated for the treatment of esophageal achalasia. We describe our initial clinical experience treating achalasia patients with POEM.

Methods. Six patients (mean age: $64 \pm 11$ years) with symptomatic achalasia were enrolled. Using flexible endoscopy a mucosal incision approximately $8 \mathrm{~cm}$ above the gastro-esophageal junction (GEJ) was performed. A submucosal esophageal tunnel was then created down to the gastric cardia using hook needle-knife cautery. Subsequently, dissection of solely the inner circular esophageal muscle fibers at the GEJ was attempted. Haemostatic clips were used to close the mucosa. Patients had postoperative barium esophagrams and routine clinical follow-up.

Results. All (6/6) patients successfully underwent POEM. Median myotomy length was $7 \mathrm{~cm}(6-12 \mathrm{~cm})$. Smooth passage of the endosocope through the GEJ was observed in all patients. Operative time ranged from 120 to $240 \mathrm{~min}$. No leaks were detected and no clinical complications were observed. Mean length of stay was $1.2 \pm 0.4$ days and all patients reported immediate symptom relief.

Conclusions. Our initial observation indicates the safety of POEM and early clinical results were promising. Although further evaluation seems mandatory, POEM could become the treatment of choice for patients with symptomatic achalasia.

\section{5}

Ist die Single Port Access-

Cholezystektomie eine sichere Alternative zum konventionell laparoskopischen Vorgehen? - Ein retrospektiver Vergleich über zwei Jahre

\section{K. Cziupka, L. Mirow}

Landkreis Mittweida Krankenhaus gGmbH, Mittweida, Germany

Grundlagen. Im Rahmen der Fortentwicklung der laparoskopischen Cholezystektomie ist eine derzeitige Bestrebung, Narben und operatives Trauma auf ein Minimum zu reduzieren. Die single port access (SPA) - Laparoskopie stellt hierbei einen Kompromiss zwischen NOTES und konventionell laparoskopischem Vorgehen dar. Dieser Bericht beschreibt die Erfahrungen eines Krankenhauses der Regelversorgung mit 69 SPA-Cholezystektomien im Vergleich zu den im selben Zeitraum durchgeführten 295 konventionell laparoskopisch operierten Cholezystektomien.

Methodik. Im Zeitraum von Januar 2009 bis Dezember 2010 wurden 295 konventionell laparoskopische und 69 SPA Cholezystektomien durchgeführt. Die Daten wurde retrospektiv erfasst und hinsichtlich demographischer Verteilung der beiden Kollektive sowie der Operationszeit, postoperativer Krankenhausaufenthaltsdauer, intraoperativer und postoperativer Komplikationsraten sowie Konversionsraten verglichen.

Ergebnisse. Die SPA Gruppe unterschied sich hinsichtlich Geschlechterverteilung, ASA-Klassifikation und Einweisungsmodus von der konventionell laparoskopisch operierten Gruppe. Keine relevanten Unterschiede bestanden in der Rate an intraabdominellen Voroperationen und der Altersverteilung. Auch die intra- und postoperativen Komplikationsraten differierten nicht signifikant. Die Schnitt-Naht-Zeit, welche in der SPA Gruppe im Laufe der Zeit abnahmen, war in der SPA Gruppe sogar signifikant kürzer, auch die postoperative Krankenhausverweildauer war niedriger.

Schlussfolgerungen. Die SPA-Cholezystektomie ist in ausgewählten Patientenkollektiven der konventionell laparoskopischen Methode ebenbürtig. Der Vorteil der besseren Kosmetik wird demzufolge nicht durch einen Mangel an Sicherheit erkauft.

\section{6}

Long time evaluation of the transvaginal access in transvaginal rigid-hybrid NOTES cholecystectomy and anterior resection in respect to morbidity, life quality and sexual function

\section{J. Janczak ${ }^{1}$, I. Tarantino ${ }^{1}$, S. Luz ${ }^{1}$, I. Siercks ${ }^{2}$, G. Linke ${ }^{3}$, R. Warschkow ${ }^{1}$, A. Zerz ${ }^{1}$ \\ ${ }^{1}$ Kantonsspital St. Gallen, Chirurgie, St. Gallen, Switzerland; ${ }^{2}$ Kantonsspital St. Gallen, Department of Gynaecology, St. Gallen, Switzerland; ${ }^{3}$ Department of General, Visceral and Transplant Surgery, University of Heidelberg, Heidelberg, Germany}

Background. Evaluation the long time outcome concerning the morbidity, quality of life and sexual function of the transvaginal access in NOTES cholecystectomy and anterior resection.

Methods. Patients with a one year follow-up were included. A pre-and two-week postoperative gynaecological examination was performed. Quality of life and sexual function was assessed pre, 6 weeks and 1 year postoperatively by the Quality of Life Index. Sexual function was evaluated by the validated Female Sexual Function Index at 1 year after the operation.

Results. From 09.2008 150 patients after tvCCE and 42 patients after tvAR had a one year follow-up. In 9 patients (4.6\%; $95 \%$ CI: 2.3-8.8\%) were found: bacterial vaginosis $(n=1)$, dehiscence of the healing wound in the posterior fornix $(n=2)$, and vulvitis/colpitis $(n=4)$, ulceration of the vaginal wall $(n=1)$, infected haematoma in the pouch of douglas $(n=1)$. All were treated conservatively except one patient. The GIQLI score improved significantly $(p<0.001)$ and complaints of painful sexual intercourse did not change over time $(p=0.240)$. Impairment of sex life decreased significantly $(p<0.001)$. 
Conclusions. The transvaginal access can safely be performed. Furthermore the transvaginal acces does not impairs sexual life or cause pain during sexual intercourse.

\section{7}

\section{Initial series of single incision laparoscopic rectum resection}

W. Brunner, J. Schirnhofer, R. Frass, N. Waldstein, K. Pimpl, C. Mittermair, C. Obrist, H. Weiss

KH Barmherzige Brüder, Salzburg, Austria

Background. Single incision laparoscopic surgery (SIL) should offer minimal scarring without compromising surgical outcome. Herein we describe our initial series of SIL rectum resection.

Methods. Between 12-2008 and 12-2010 we performed 14 rectum resections for carcinoma out of a total number of 130 patients undergoing SIL colorectal surgery (Mean $\pm \mathrm{SD}$ age $53 \pm 13$ years, BMI $25 \pm 6 \mathrm{~kg} / \mathrm{m}^{2}$, sex $10 \mathrm{f} / 4 \mathrm{~m})$. Two patients underwent neoadjuvant chemoradiation. The entire operation was carried out via the ileostomy position (8) or transumbilically (6) using a single port system.

Results. All but two procedures could be completed without adding any additional trocar. Adhesions were found in 9 patients. Specimen retrieval was carried out trough the port site, transrectal (2) or transvaginal (1). Anastomosis was completed by means of transanal stapling (10) or coloanal suturing (4). Operation time lasted (Mean) 220 min. Mean specimen length and number of lymphnodes yielded $29 \mathrm{~cm}$ and 15, respectively. Patients started oral intake and mobilization at the day of surgery. Two patients had to be reoperated, one for anastomotic leak, one for R1 resection of an alternate tumor. Patients were discharged on day 11 (7-27).

Conclusions. Singe port laparoscopic rectum resections is feasible and can be carried out via transumbilical or ileostomy site approach. This further reduces the surgical trauma.

\section{8}

Pure natural orifice transrectal surgery for oncologic resection of the rectosigmoid compared to the conventional laparoscopic-assisted approach

\section{E. Rieder ${ }^{1,2}$, G. O. Spaun ${ }^{2}$, Y. S. Khajanchee ${ }^{2}$, D. V. Martinec $^{2}$, B. N. Arnold ${ }^{2}$, L. L. Swanstrom ${ }^{2}$, M. H. Whiteford ${ }^{2}$}

${ }^{1}$ Department of Surgery, Medical University of Vienna, Vienna, Austria; ${ }^{2}$ Legacy Health, MIS Program, Portland, OR, USA
Background. A transrectal (TR) approach makes immense sense for colorectal surgery as the colotomy is incorporated into subsequent anastomosis. Aim of this study was to assess whether pure TR recto-sigmoidectomy can be performed with adherence to oncological principles similar to a standard laparoscopic-assisted approach (LAP).

Methods. Human cadavers were allocated to either TR $(n=4)$ or LAP $(n=2)$. A sigmoid lesion was created. Transrectal retrograde mobilization of the recto-sigmoid was performed using transanal endoscopic microsurgery (TEM) instrumentation. After ligation of the superior hemorrhoidal artery and further mobilization, the specimen was delivered transanally. NOTES colorectal anastomosis was attempted. Lymph node yield, resection margins, and operative time were compared to LAP.

Results. Transrectal rectosigmoid-dissection was achieved in all (4/4) attempts and revealed similar numbers of lymph node (median: 5) compared to the LAP group (median: 4.5). One pure TR-approach failed to resect the lesion. Three TR-procedures required laparoscopic mobilization to provide adequate margins. Transrectal resected specimens were $16 \pm 4 \mathrm{~cm} v s .31 \pm$ $9 \mathrm{~cm}$ achieved by LAP $(p<0.01)$. TR operative time was significantly longer $(247 \pm 15 \mathrm{~min} v s .110 \pm 14 \mathrm{~min})$.

Conclusions. Lymph node yield during TR-sigmoidectomy was similar to the LAP approach. However, conventional TEM instrumentation did not allow adequate colon mobilization, which has to be addressed in additional studies.

\section{ACO/ASSO - Österreichische Gesellschaft für Chirurgische Onkologie: Multimodale Konzepte und individualisierte Krebstherapie}

\section{5}

Gene expression profiling in colon cancer: improvement of recurrence risk assessment

\footnotetext{
M. Knauer ${ }^{1}$, T. Bachleitner-Hofmann ${ }^{2}$, R. Bartsch ${ }^{2}$, A. Haid ${ }^{1}$, E. Wenzl ${ }^{1}$, J. Tabernero ${ }^{3}$, R. Rosenberg ${ }^{4}$, M. Lutke-Holzik ${ }^{5}$, F. Bibeau ${ }^{6}$, J. van der Hoeven ${ }^{7}$, R. Salazar 8

${ }^{1}$ Academic Teaching Hospital, Feldkirch, Austria; ${ }^{2}$ Medical University, Vienna, Austria; ${ }^{3}$ Vall d'Hebron University Hospital, Barcelona, Spain; ${ }^{4}$ Klinikum Rechts der Isar, Technical University, Munich, Germany; ${ }^{5}$ Medisch Spectrum Twente, Enschede, Netherlands; ${ }^{6} \mathrm{CRLC}$ Val d'Aurelle, Montpellier, France; ${ }^{7}$ Medisch Centrum, Alkmaar, Netherlands; ${ }^{8}$ Instituto Catala d'Oncologia, Barcelona, Spain
} 
Background. Recurrence risk for colon cancer (CC) varies according to clinico-pathological stage. While adjuvant chemotherapy is routinely recommended by therapeutic guidelines for stage III cancer, it remains controversial in stage II cancer. Gene expression profiling is likely to provide more reliable information on prognosis and response to chemotherapy.

Methods. Several multigene assays have been developed to improve outcome prediction, either based on realtime-PCR from formalin-fixed tissue or RNA-microarrays from frozen tissue. The 18-gene ColoPrint signature was developed from 188 frozen tumor samples from three Dutch hospitals and is now being prospectively evaluated in the multicenter PARSC-study, which is recruiting 600 stage II colon cancers.

Results. The ColoPrint signature was the most powerful predictor of 5-year distant disease-free survival in the training cohort (HR 3.4, $p<0.001$ ) as well as in two independent validation studies on 114 stage II patients from Barcelona (HR 3.3, $p=0.017$ ) and 135 stage II patients from Munich (HR 4.1, $p=0.009$ ). By October 2010, more than 700 patients have been recruited for the PARSC-study in 23 centers worldwide.

Conclusions. Gene expression profiling is likely to improve our knowledge about recurrence risk and hopefully also the estimation of benefit from adjuvant chemotherapy in stage II colon cancer.

\section{6}

\section{Repetitive bone marrow assessment of circulating cytokeratin 18 fragment M65 in colorectal cancer patients}

\section{Buxhofer-Ausch ${ }^{1}$, U. Olszewski ${ }^{1}$, G. Hamilton ${ }^{1}$, S. Kriwanek ${ }^{1,2}$, C. Ausch ${ }^{1,2}$ \\ ${ }^{1}$ Cluster für Translationale Onkologie Ludwig Boltzmann Forschungsgesellschaft, Wien, Austria; ${ }^{2}$ Chirurgische Abteilung Donauspital SMZ Ost, Wien, Austria}

Background. Recently we identified M65 as serum marker likely to be valuable to identify patients with incidence of systemic disease in colorectal cancer. The study aimed to determine if changes of M65 expression in repetitive bone marrow measurements correlate with clinical parameters and 5 year follow up.

Methods. Repetetive BM assessments (preoperative, $+1 \mathrm{y},+2 \mathrm{y})$ were available for 35 colorectal cancer patients and 15 controls. The concentration of M65/total soluble CK 18 fragments was determined using a M65 ELISA.

Results. In the group without evidence of relapse (n: 26) 17 patients exhibited a significant decrease of M65 postoperatively $(+1 \mathrm{y},+2 \mathrm{y})$ in response to tumor removal, in contrast to nine patients who revealed persistent or higher M65 levels postoperatively. In patients with relapse (n: 9) M65 levels increased or were persistent in 5 patients and decreased in 4 . Correlation with relapse and survival will be presented at the meeting.

Conclusions. Removal of the tumor in colorectal cancer patients resulted in a reduction in circulating CK 18 fragments in the bone marrow in the majority of patients. Persistent or increased postoperative levels of CK18 in the bone marrow point to disseminated tumor cells as a putative source of CK 18 fragments in these patients.

\section{7}

\section{Klinische Bedeutung von CAIX und Her-2 Überexpression beim Ösophaguskarzinom}

\section{G. Jomrich, B. Jesch, P. Birner, S. F. Schoppmann}

Universitätsklinik für Chirurgie, Vienna, Austria

Grundlagen. Die Carboanhydrase IX, eine transmembranes Glycoprotein, scheint eine wesentliche Rolle bei der Hypoxie-Anpassung von Tumorzellen an zu spielen.

Methodik. In dieser Studie wurde die Expression von CAIX und HER-2 bei 330 PatientInnen mit gastroösophagealen Karzinomen (182 ACs und 148 SCCs) untersucht. In 137 Fällen wurden weiters die korrespondierenden Lymphknotenmetastasen, in 34 Fällen die Fernmetastasten und in 14 Fällen die Lokalrezitive bezüglich CAIX Expression untersucht. 147 Fälle $(44,5 \%)$ zeigten starke CAIX Expression (AC 46,7\%; ACC 41,9\%).

Ergebnisse. Der CAIX Status des Primärtumors beeinflusste die CAIX Expression der entspechenden Lymphnotenmetastasen ( $p<0.001$, linear regression). Eine hohe CAIX Expression war ein unabhängiger prognostischer Faktor für ein kürzeres Gesamtüberleben, wie auch für ein krankheitsfreies Überleben ( $p<0.05$, Cox regression). 29 ACs $(15,9 \%)$ und 6 SCCs $(4,1 \%)$ zeigten HER-2 Überexpression. Bei AC wurde ein signifikanter Zusammenhang zwischen HER-2 Status und CAIX Expression gefunden ( $p=0.009$, Chi Quadrat Test).

Schlussfolgerungen. Eine CAIX-Überexpression steht in Zusammenhang mit kürzerem Überleben bei gastroösophagealen Karzinomen. Dementsprechend dürfte der hypoxische Phenotyp während der Bildung von Lymphknotenmetastasen erhalten werden. Die Hemmung von CAIX reduziert möglicherweise die Entstehung von Metastasen. Bei HER-2 überexprimierenden ACs könnte das Blockieren dieser Tyrosinkinase (z.B. durch monoklonale Antikörper) einen derartigen Effekt induzieren. 


\section{8}

Implementierung vom Mammaprint

im klinischen Alltag zur

Risikoeinschätzung bei Patientinnen

mit hormonrezeptorpositivem

Mammakarzinom - erste

Anwendungserfahrungen

R. Exner ${ }^{1}$, Z. Horvath ${ }^{2}$, M. Rudas ${ }^{2}$, U. Pluschnig ${ }^{3}$, S. Bigenzahn ${ }^{1}$, F. Fitzal ${ }^{1}$, M. Gnant ${ }^{1}$, P. Dubsky ${ }^{1}$

${ }^{1}$ Univeristätsklinik für Chirurgie, Wien, Austria;

${ }^{2}$ Univeristätsklinik für Pathologie, Wien, Austria;

${ }^{3}$ Univeristätsklinik für Innere Medizin I, Wien, Austria

Grundlagen. Nach den St. Gallen Kriterien von 2009 sollen validierte Genexpressionsanalysen im klinischen Alltag implementiert werden, um zusätzlich zu den klassischen Tumor- und Patienteneigenschaften wie Grading, Staging, Nodalstatus und Hormonrezeptorexpression eine Risikoeinschätzung und Empfehlung für bzw. gegen eine Chemotherapie geben zu können. Ziel unserer Studie ist es, den Einfluß von Mammaprint auf die Entscheidung im interdisziplinären Tumorboard über die weitere Therapie bei Patientinnen mit hormonrezeptorpositivem Mammakarzinom zu evaluieren.

Methodik. Bei Patientinnen mit bioptisch verifiziertem primärem MammaKarzinom (pT1c-pT2, G1-G2, N0-N1, ER und/oder PR positiv, Her 2 negativ) wird nach der Resektion Tumorgewebe vom zuständigen Pathologen zur Genexpressionsanalyse durch die Firma Agendia gesandt. Nach Vorliegen der postoperativen Histologie wird im Tumorboard Therapieempfehlung abgegeben, diese wird nach Vorliegen des Mammaprintergebnisses erneut diskutiert.

Ergebnisse. Beginn der Studie war 4/2010, die Teilnahme von 75 Patientinnen ist geplant. Derzeit wurden 21 Patientinnen eingeschlossen und in low bzw. high risk durch Mammaprint eingestuft. Wir werden über die Ergebnisse und die Entscheidungsänderung hinsichtlich einer adjuvanten Chemotherapie aufgrund des Mammaprint-Ergebnisses berichten.

Schlussfolgerungen. Validierte Genexpressionsanalysen zur Risikoevaluation bei Patientinnen mit hormonrezeptorpositivem Mammakarzinom können als zusätzliche Entscheidungshilfe des interdiziplinären Tumorboards für oder gegen eine adjuvante Chemotherapie hilfreich sein, kontrollierte Studien zur klinischen Anwendung sind derzeit im Gange.

\section{9}

Zirkulierende Endothelzellen und phänotypisch verwandte Zellpopulationen zeigen ein prädiktives Potential in der Bevacizumab basierten Tumortherapie

P. Starlinger, P. Brugger, C. Reiter, D. Schauer,

S. Sommerfeldt, D. Tamandl, I. Kührer, S. Schoppmann, M. Gnant, C. Brostjan

Universitätsklinik für Chirurgie, Wien, Austria

Grundlagen. Die Inaktivierung von VEGF (vascular endothelial growth factor) durch den Antikörper Bevacizumab konnte in diversen Tumorentitäten therapeutische Erfolge erzielen. Dennoch sprechen viele Patienten nicht auf die anti-angiogenetische Therapie an, und die Identifizierung von Markern, die den Erfolg der Therapie vorhersagen, ist von großer Relevanz. Zirkulierende Endothelzellen (CECs) wurden daher in dieser Studie auf ihr prädiktives Markerpotential evaluiert.

Methodik. 20 Patienten mit lokal fortgeschrittenem Pankreaskarzinom wurden neoadjuvant mit vier Zyklen Gemcitabin sowie zweiwöchentlich mit Bevacizumab behandelt. Zirkulierende Zellpopulationen wurden mittels Durchflusszytometrie im Patientenblut anhand der CD45, CD31 und CD146 Expression evaluiert.

Ergebnisse. Im Verlauf unserer Analysen war es uns möglich, drei phänotypisch verwandte Zellpopulationen zu unterscheiden. Jede dieser Populationen zeigte einen distinkten Verlauf und zwei der drei Zellpopulationen korrelierten hoch signifikant mit dem Ansprechen der Patienten auf die Therapie.

Schlussfolgerungen. Mit dieser Studie ist es erstmals gelungen, drei Zellpopulationen mit überlappendem Phänotyp mittels Durchflusszytometrie klar von einander abzugrenzen und sie CECs, aktivierten T-Zellen und großen Thrombozyten zuzuordnen. Die Analyse dieser Zellpopulationen stellt einen vielversprechenden Ansatz dar, um das Ansprechen von Patienten auf die kombinierte Bevacizumab Therapie vorauszusagen und damit eine individualisierte Behandlung zu ermöglichen.

\section{0}

Funktionelle Wundheilung trotz drastischer VEGF Inaktivierung nach neoadjuvanter Bevacizumab Therapie

\author{
L. Alidzanovic, P. Starlinger, D. Schauer, T. Maier, \\ C. Nemeth, B. Herberger, L. Pop, B. Grünberger, \\ T. Grünberger, C. Brostjan \\ Universitätsklinik für Chirurgie, Wien, Austria
}

Grundlagen. Der Gefäßwachstumsfaktor VEGF spielt sowohl in der Tumorangiogenese als auch bei der physiologischen Wundheilung eine zentrale Rolle. Dem Ein- 
satz von Bevacizumab, einem VEGF inaktivierenden Antikörper, im Rahmen der neoadjuvanten Chemotherapie wurde nachgesagt, dass es vermehrt $\mathrm{zu}$ postoperativen Wundheilungsstörungen komme. Dies wird bis dato sehr kontroversiell diskutiert und mechanistische Studien fehlen weitgehend.

Methodik. Bei 23 Patienten mit kolorektalen Lebermetastasen wurden nach neoadjuvanter Chemotherapie mit bzw. ohne Bevacizumab im perioperativen Zeitraum Angiogenesefaktoren in Plasma und Wundflüssigkeit analysiert. Leberresektionen wurden 5 Wochen nach der letzten Bevacizumab Therapie durchgeführt.

Ergebnisse. Wundheilungsstörungen traten bei 2 Patienten auf (jeweils mit bzw. ohne Bevacizumab Therapie). Generell zeigten sich in der Wunde höhere Konzentrationen an Angiogenesefaktoren als im Plasma, wobei Bevacizumab therapierte Patienten deutlich geringere VEGF Spiegel aufwiesen als Patienten ohne Bevacizumab. Das zirkulierende VEGF in Wundflüssigkeit und Plasma der Bevacizumab Patienten war zudem $\geq 90 \%$ Antikörper gebunden. Interessanter Weise war der Wachstumsfaktor PD-ECGF in Wundflüssigkeit von Bevacizumab Patienten substanziell erhöht.

Schlussfolgerungen. Nach neoadjuvanter Bevacizumab Therapie ist VEGF zum Zeitpunkt der Operation großteils Antikörper-gebunden und daher inaktiv. Dies ist sowohl systemisch als auch in der lokalen Wundflüssigkeit nachzuweisen. Die Wundheilung scheint demnach ohne freies VEGF stattzufinden, d.h. der VEGF Verlust könnte durch andere Wachstumsfaktoren wie PD-ECGF kompensiert sein.

\section{Arbeitsgemeinschaft für Qualitätssicherung in der Chirurgie: Qualitätsverbessernde Maßnahmen im Krankenhaus}

\section{5}

\section{Präoperative Risikostratifizierung - Möglichkeiten und Limitationen eines} interdisziplinär erhobenen Risikoscores

\author{
M. Mille ${ }^{1}$, G. Burgard ${ }^{2}$, A. Stier ${ }^{1}$ \\ ${ }^{1}$ HELIOS Klinikum Erfurt, Klinik für Allgemein- \\ und Viszeralchirurgie, Erfurt, Germany; ${ }^{2}$ HELIOS Klinikum \\ Erfurt, Klinik für Anästhesie, Intensivmedizin und \\ Schmerztherapie, Erfurt, Germany
}

Grundlagen. Der Erfolg eines chirurgischen Eingriffes hängt unter anderem ganz wesentlich von der Erhebung des perioperativen Risikos ab. Zur Einschätzung der perioperativen Morbidität und Mortalität wird seit Mitte 2008 an unserem Klinikum der Maximalversorgung ein präoperativer Risikoscore verwendet, welcher gemeinsam vom Chirurgen und Anästhesisten erhoben wird. Dabei wird ein entsprechendes kardiopulmonales Risikolevel nach Punktevergabe festgelegt, welches diagnostische bzw. therapeutische Maßnahmen mit sich zieht. Es soll nun anhand der Mortalität versucht werden diesen Risikoscore zu bewerten, sowie dessen Möglichkeiten und Grenzen aufzuzeigen.

Methodik. Sämtliche Fälle an unserer Klinik für Allgemein- und Viszeralchirurgie in einem Auswertungszeitraum von zwei Jahren $(n=4236)$ wurden retrospektiv anhand ihres präoperativ erhobenen Risikoscores analysiert.

Ergebnisse. In diesem Zeitraum traten 161 Todesfälle auf, wobei $8 \%$ ein niedriges, $11 \%$ ein mittleres und $81 \%$ der Patienten ein hohes Risiko aufwiesen. Haupttodesursache bei Patienten mit einem hohen Risikoscore war dabei die Sepsis. Kardiopulmonale Ursachen fanden sich dagegen nur noch in ausgewählten Fällen.

Schlussfolgerungen. Der verwendete Risikoscore stellt eine gute Basis dar, um das kardiopulmonale Risikoprofil eines Patienten zu evaluieren. Die Entscheidung für den Eingriff und die Einschätzung des Gesamtrisikos lässt sich jedoch nicht anhand eines einzigen Risikobogens fällen. Weitere Ergänzungen sind notwendig, um das perioperative Risiko noch besser einschätzen zu können.

\section{6}

\section{Team Time Out (TTO) - ein notwendiger Beitrag zur Patientensicherheit in der Kinderchirurgie}

\section{A. Haberlik}

Univ. Klinik für Kinder- und Jugendchirurgie Graz, Graz, Austria

Behandlungsfehler in Krankenhäusern stehen immer im Fokus der Öffentlichkeit, da schwerwiegende Gesundheitsfolgen für die Betroffenen resultieren können. Daher ist es entscheidend potenzielle Fehlerquellen zu analysieren und gezielt Mechanismen für die Prävention zu entwickeln. Bisher wurden an unserer Klinik standardisiert drei Schritte für die Sicherheit und Erkennung von Patienten vor operativer Therapie durchgeführt. 1: Patientenidentifikation, 2: Markierung des Eingriffsortes (Seite) und 3: Zuweisung zum richtigen OP-Saal. Am 1. Juli 2009 wurde mit der Implementierung des „Team Time Out“ (TTO) ein weiterer Schritt in der Prävention von Eingriffsverwechslungen gesetzt. Das TTO bedeutet ein „letztes Innehalten" direkt vor dem Schnitt mit nochmaliger standardisierter Abfrage der Identität des Patienten, der Diagnose (Seite) und des geplanten Eingriffes. Auf Grundlage der Joint Commission on Accreditation of Healthcare Organizations wurde eine „Checkliste“ entworfen und inhaltlich an die Bedürfnisse unserer OP-Einheit angepasst. Die Dokumentation des TTO erfolgt direkt auf der Operationseinwilligung. Das TTO behindert den Ablauf im OP nicht und gibt letztlich den Verantwortlichen die Gewissheit, Fehlerquellen ausgeschlossen zu haben. Das kurze Innehalten in einer routinemäßig ablaufenden Behandlungslo- 
gistik wurde ein wesendliches Instrument zur Vermeidung von Informationsdefiziten und von Verwechslungen und ist ein wichtiger Faktor für die Patientensicherheit.

\section{8}

\section{Lernen unter Berücksichtigung der Patientensicherheit}

\section{S. Kriwanek}

\section{SMZ Ost Donauspital, Wien, Austria}

Grundlagen. Die Voraussetzungen der Ausbildung zum Facharzt für Chirurgie haben sich in den letzten 10 Jahren deutlich verändert. Das Interesse der Lernenden, die notwendige Expertise zu erlangen, steht den Bedürfnissen der Patienten nach einer Betreuung durch erfahrene Therapeuten entgegen. Moderne Ausbildungssysteme haben die Aufgabe Methoden zu entwickeln, die ein Lernen ohne Gefährdung der Patienten ermöglichen.

Methodik. Alle Verfahren, die zum Einsatz kommen, haben das Ziel, die Auszubildenden wesentlich besser auf ihre Aufgabe vorzubereiten, als dies in der Vergangenheit der Fall war. Dadurch kann die Lernkurve verkürzt werden und zum Teil vor dem eigentlichen operativen Geschehen stattfinden. Folgende Methoden eignen sich für diesen Zugang: Erlernen von Grundtechniken am Modell, Gliedern eines Eingriffs in verschiedene Abschnitte, mentale Vorbereitung auf spezifische Risken und Gefahrensituationen, sowie auf den Umgang mit unerwarteten Ereignissen (wie einer massiven Blutung), Simulatortraining und der praktische Unterricht von chirurgischen Techniken mit der Vierschritt Methode.

Schlussfolgerungen. Durch den Einsatz moderner Lehrmethoden in der Chirurgie ist es möglich, angehende Chirurgen und Chirurginnen wesentlich besser auf die Planung und Durchführung operativer Tätigkeit vorzubereiten und damit die Sicherheit der Patienten $\mathrm{zu}$ gewährleisten.

\section{Österreichische Gesellschaft für Thorax- und Herzchirurgie: Herzchirurgie bei Erwachsenen}

\section{9}

Five years experience with the stentless Sorin Freedom Solo aortic prosthesis in 139 patients

M. Thalmann ${ }^{1}$, N. Taheri ${ }^{1}$, J. Kaiblinger ${ }^{1}$, K. Kornigg ${ }^{1}$, H. Pisarek ${ }^{1}$, F. Veit ${ }^{1}$, G. H. Sodeck ${ }^{2}$, M. Gorlitzer ${ }^{1}$, M. Grabenwöger ${ }^{1}$
${ }^{1} \mathrm{HZH}-$ Hietzing, Vienna, Austria; ${ }^{2}$ Inselspital, Swiss Cardiovascular Center, Bern, Switzerland

Background. Sorin Freedom Solo is a stentless pericardial aortic valve which is supra-annular seated for best hemodynamic performance. We report our experience and mid term follow-up data of 139 patients (mean age 75 years, log EUROSCORE: 11.3) who underwent aortic valve replacement (AVR) from 01/2006 till $12 / 2010$

Methods. Patients had been operated for isolated AVR as well as for concomitant procedures (45\%). Echocardiographic measurements (TTE) were performed before discharge and after a follow-up period up to 36 month postoperatively (mean follow-up 13 month).

Results. Valve sizes from $19 \mathrm{~mm}$ to $27 \mathrm{~mm}$ were implanted. (19 mm: 2, $21 \mathrm{~mm}: 37,23 \mathrm{~mm}: 48,25 \mathrm{~mm}$ : 50, $27 \mathrm{~mm}$ : 3). LVOT velocities and gradients for different valve sizes are listed in the table (postoperatively/follow-up TTE). Observed mortality was $5.7 \%$, for isolated valve replacement $4.1 \%$ respectively.

Conclusions. The hemodynamic performance of the Sorin Freedom Solo aortic valve is excellent. Mid-term follow-up cardiographic evaluations were excellent regarding hemodynamic parameters. As in all new valve types late performance and durability of the valve have to be assessed.

\begin{tabular}{lccc}
\hline \multicolumn{4}{c}{ hemodynamic data } \\
\hline valve size & $21 \mathrm{~mm}$ & $23 \mathrm{~mm}$ & $25 \mathrm{~mm}$ \\
\hline $\mathrm{V}_{\max }(\mathrm{m} / \mathrm{s})$ & $2.1 / 2.0$ & $2.1 / 2.0$ & $1.9 / 1.8$ \\
\hline $\mathrm{AV}$ max $\mathrm{PG}(\mathrm{mmHg})$ & $19.6 / 16.9$ & $18.1 / 15.2$ & $14.6 / 13.3$ \\
\hline $\mathrm{AV}$ & $11.2 / 10.3$ & $10.0 / 8.7$ & $8.1 / 8.0$ \\
\hline
\end{tabular}

\section{0}

Tricuspid valve repair in patients supported with left ventricular assist devices

\section{Schweiger, E. Potapov, A. Stepanenko, J. Vierecke, M. Kukucka, T. Krabatsch, R. Hetzer \\ Deutsches Herzzentrum Berlin, Berlin, Germany}

Background. In end stage heart failure patients severe tricuspid regurgitation is one of the signs of biventricular failure with subsequent need for biventricular support (BVAD). Tricuspid valve repair (TVR) may avoid BVAD.

Methods. Consecutive patients with tricuspid regurgitation of grade $3+$ receiving either LVAD with concomitant tricuspid valve repair (Group A, $n=7$ ) or a BVAD or TAH (Group B, $n=17$ ) implanted in our institution between 2007 and 2010 were compared retrospectively. Intermacs stage I, age below 18 years, postcardiotomy failure and graft failure were exclusion criteria. 
Results. Preoperative parameters were similar in both groups. No differences in 30 day mortality (33.3 vs. 23.5\%, $p=0.8$ ), postoperative dialysis, ICU stay (35 vs. 22 days, $p=0.94$ ) or ventilation time (15 vs. 13 days) were seen. Timing of discharge home was similar in both groups ( $50 \%$ after a median of 45 days $v$ s. $53 \%$ after a median of 40 days $(p=0.78)$. Two weeks after surgery no significant TVR had been seen in group A. Right ventricular failure requiring mechanical support occurred in one patient in group A.

Conclusions. In patients with tricuspid regurgitation of grade 3+ LVAD support combined with TVR showed comparable results to biventricular support with regard to short-term outcome.

\section{1}

\section{Benefit of bilateral skeletonized internal thoracic arteries regarding sternal complications in arterial revascularization}

\section{A. Sakic, N. Fischler, O. Chevtchik, R. Schistek, M. Grimm, E. Ruttmann-Ulmer \\ Department of Cardiac Surgery, Innsbruck, Austria}

Background. Limited blood supply to the thoracic chest wall is a known risk factor for sternal wound complications after CABG surgery. Therefore, bilateral internal thoracic arteries are still rarely utilized even due to proven superior graft patency.

Methods. All 369 patients receiving bilateral internal thoracic artery CABG procedures (BITA) from $01 / 2001$ to $12 / 2010$ were analyzed for sternal wound complications. Possible protective surgical strategies were investigated for their effect on the occurrence of sternal wound complications adjusted for known risk factors and relevant comorbidities.

Results. Sternal wound complications occured in 25 patients $(6.8 \%)$, with a sternal dehiscence rate of $2.7 \%$. In multivariate analysis, diabetes (HR: 3.3, 95\% CI: 1.2-4.7; $p=0.02$ ) and pedicled preparation of BITA (HR: $4.6,95 \%$ CI: $1.8-11.7 ; p=0.001$ ) were risk factors for sternal complications. Additionally, skeletonized BITA was further protective to avoid sternal dehiscence (HR: 14.6, 95\% CI: $2.8-78 ; p=0.002$ ).

Conclusions. Adjusted for common risk factors, skeletonization of BITA is the most effective strategy to avoid sternal wound complications.

\section{2}

Cardiac-event free survival in multiple arterial revascularisation - propensityscore matched analysis of radial vs. right internal thoracic artery graft as second conduit of choice

\section{E. Ruttmann-Ulmer ${ }^{1}$, N. Fischler ${ }^{1}$, A. Sakic ${ }^{1}$, O. Chevtchik ${ }^{1}$, R. Schistek ${ }^{1}$, H. Ulmer ${ }^{2}$, A. M. Strasak ${ }^{2}$, M. Grimm ${ }^{1}$ \\ ${ }^{1}$ Department of Cardiac Surgery, Innsbruck, Austria; ${ }^{2}$ Department of Medical Statistics, Informatics and Health Economy, Innsbruck, Austria}

Background. The second best arterial conduit for cardiac revascularization is still a matter of debate. Aim of our study was to investigate the outcome of either radial artery (RA) or right internal thoracic artery (RITA) for MAR.

Methods and results. All first, non-emergent multivessel CABG patients receiving more than 1 arterial conduit were studied. Patients were grouped according to the conduits used as bilateral internal thoracic artery and concomitant SVG (BITA + SVG, 277 patients) and LITA+ RA (left internal thoracic artery and RA with/without concomitant SVG, 724 patients). Propensity-score matched analysis was performed to reduce the impact of treatment selection.

In both the unselected and matched population, the risk for perioperative MACCE was significantly lower in the BITA + SVG group.

Survival was significantly higher in the BITA + SVG group ((HR): 0.23 ; 95\% CI: 0.066-0.81; $p=0.022$ ). Furthermore, the cardiac-event free survival was significantly higher in the BITA+SVG group (HR: 0.19; 95\% CI: $0.09-$ $0.41 ; p<0.001)$.

Conclusions. The results of our study provide strong evidence for the superiority of a RITA graft for revascularisation. Lower MACCE in BITA patients might be associated with less aortic manipulation and lower vasospasm of ITA grafts.

\section{3}

Outcome of conduits in symptomatic patients after multiple arterial cardiac revascularization - an angiographic study

N. Fischler ${ }^{1}$, A. Sakic ${ }^{1}$, O. Chevtchik ${ }^{1}$, R. Schistek ${ }^{1}$, H. Ulmer ${ }^{2}$, M. Grimm', E. Ruttmann-Ulmer ${ }^{1}$

${ }^{1}$ Department of Cardiac Surgery, Innsbruck, Austria; ${ }^{2}$ Department of Medical Statistics, Informatics and Health Economy, Innsbruck, Austria 
Background. Cardiac revascularization using a radial artery (RA) or second internal thoracic artery (BITA) is predominately reserved to younger patients.

Methods. All 1001 patients receiving a second ITA graft or a RA between 2001 and 2010 were studied. In the follow-up, 107 patients $(10.7 \%)$ underwent a total of 118 repeated cardiac catheterizations. Angiographic outcomes were classified as (1) occluded, (2) severly diseased ( $\geq 70 \%$, string sign) or (3) patient $(<70 \%)$. A total of 313 conduits (132 ITA grafts, 95 RA and 86 SVG) were investigated.

Results. Repeated cardiac catheterization was necessary in $6.8 \%$ of patients receiving BITA + SVG grafts and in $12.2 \%$ of patients with RA $(p=0.02)$ after a median follow-up of 26.9 months (0.0-101.2 months). In the BITA + SVG group, $26.3 \%$ showed graft occlusion/disease of at least 1 graft compared to $60.2 \%$ of patients receiving RA $(p=0.007)$. Occlusion or severe diseased ITA grafts in $10.2 \%$, SVG $20.9 \%$ ( $p=0.02$ ) were diagnosed. The highest disease rate showed RA grafts with $37.9 \%$ and were significantly lower than for ITA $(p<0.001)$ or SVG $(p=0.014)$.

Conclusions. In symptomatic patients, RA showed significantly higher disease than ITA or even SVG. In the follow-up, ITA and SVG graft patency seems to be superior to RA.

\section{4}

\section{Risk factors and outcome in aortic root replacement}

\section{F. Weidinger, N. Bonaros, J. Nagiller, S. Metzler, M. Grimm, T. Schachner}

Universitätsklinik für Herzchirurgie Innsbruck, Innsbruck, Austria

Background. We analyzed perioperative outcome in patients undergoing aortic root and ascending aortic replacement in terms of a modified Bentall Operation, or aortic root replacement using a biological prosthesis.

Methods. Two hundred and eleven patients, $81 \%$ male, aged 58 (15-84) underwent aortic root replacement and replacement of the ascending aorta. Concomitant procedures were (partial) replacement of the aortic arch in $17 \%$ and CABG in $11 \%$. $26 \%$ were operated because of acute aortic dissection. Replacement with a mechanically valved composite graft was performed in 170 patients, 41 patients underwent biological root replacement.

Results. Overall hospital mortality was $9 \%$, and $3 \%$ in elective cases. Factors significantly associated with hospital mortality were: acute aortic dissection $(p=0.007)$, coronary artery disease $(p=0.005)$, total operative time $(p=0.001)$, deep hypothermic circulatory arrest $(p=0.024)$, revision due to bleeding $(p=0.02)$ and postoperative hemofiltration $(p<0.001)$. Stroke rate was $5 \%$, significantly associated with acute aortic dissection
( $p=0.009)$, deep hypothermic circulatory arrest $(p=0.001)$ and ventilation time $(p=0.035)$.

Conclusions. Aortic root replacement using the modified Bentall technique with mechanically or biologically valved conduits or a porcine aortic root show satisfactory perioperative results with an acceptable hospital mortality.

\section{5}

\section{Retrograde Aortendissektion Typ A nach Stenting einer Aortendissektion Typ B - was tun?}

\section{G. Weiss, M. Gorlitzer, S. Folkmann, R. Moidl, F. Waldenberger, M. Grabenwoeger}

KH Hietzing, Wien, Austria

Grundlagen. Die retrograde Aortendissektionen Typ A ist bei der Stentgraftversorgung einer Typ B Dissektion eine mögliche und schwerwiegende Komplikation.

Methodik. Nach Auftreten dieser Komplikation wurden unsere Patienten in eine offene Operation konvertiert und im Kreislaufstillstand in moderater Hypothermie mit selektiver antegrader Hirnperfusion eine Jotec Evita Open Prothese implantiert. Der gestentete Teil wird über den eröffneten Aortenbogen in die Aorta deszendens vorgeschoben, anschließend wird mit der Dacron Hälfte der Aortenbogen ersetzt. Zwischen 2005 und 2010 wurden 29 Patienten aufgrund einer Aortendissektion Typ B mit einer thorakalen Stentprothese behandelt. Drei davon $(10,23 \%)$ mussten wegen einer periinterventionell aufgetretenen retrograden Aortendissektion Typ A akut in eine offene Operation konvertiert werden.

Ergebnisse. Alle Patienten überlebten den Eingriff ohne neurologische Defizite. Eine Computertomographie der thorakalen Aorta wurde innerhalb der ersten zwei Wochen, dann im Abstand von 3, 6 und 12 Monaten durchgeführt. Nach 3 Monaten zeigte sich bei allen Patienten eine komplette Thrombosierung des falschen Lumens bis auf Höhe des Stentgrafts, der Durchmesser der thorakalen Aorta war in den Verlaufskontrollen bei allen Patienten stabil.

Schlussfolgerungen. Bei noch geringer Fallzahl zeigen die positiven Ergebnisse, dass der simultane chirurgische und endovaskuläre Zugang für die Therapie der retrograden Typ A Dissektion nach endovaskulärer Stentimplantation bei Dissektion Typ B eine gute Behandlungsmöglichkeit darstellt. 


\section{6}

Auf dem Weg zur beinahe „schmerzlosen Standard-Herzchirurgie" - die Grazer Erfahrungen mit einer ergebnisorientierten externen Zertifizierung

R. Hetterle ${ }^{1}$, H. Mächler ${ }^{1}$, A. Yates ${ }^{1}$, M. Anelli Monti ${ }^{1}$, E. Beran ${ }^{1}$, W. Toller ${ }^{2}$, A. Sandner Kiesling ${ }^{2}$, K. Rieger ${ }^{1}$, B. Zirngast ${ }^{1}$, S. Özkan ${ }^{1}$, R. Lang ${ }^{1}$, C. Foussek ${ }^{3}$, K. Tscheliessnigg ${ }^{1}$

${ }^{1}$ MUG/Univ. Klinik Chirurgie/Herzchirurgie, Graz, Austria; ${ }^{2} \mathrm{MUG} /$ Anästhesiologie u. Intensivmedizin, Graz, Austria; ${ }^{3}$ Stabstelle QM-RM LKH Univ.Klinikum, Graz, Austria

Grundlagen. Obwohl in einem Vorprojekt $99 \%$ der Patientinnen glaubten, dass alles Notwendige zur Schmerztherapie getan wurde, klagten noch immer $34 \%$ über starke Schmerzen. Nicht nur in Anbetracht von Alternativen zu chirurgischen Klappenoperationen wird die möglichst schmerzlose Standard-Herzchirurgie immer wichtiger, da der erwartete perioperative Schmerz für manche PatientInnen zum alleinigen Entscheidungskriterium für eine alternative Methode werden kann.

Methodik. Struktur- und Prozesskriterien wurden aufbereitet bzw. überarbeitet, die für ein optimiertes perioperatives Schmerzmanagement notwendig sind.Dazu wurden interdisziplinäre Teams gebildet, Patientenaufklärungsfolder, eine strukturierte Anamnese, eine standardisierte mind. $3 \times$ tgl. zu erhebende Schmerzmessung, Schmerzdokumentation, Klinikumeinheitliche Interventionsgrenzen und darauf aufbauende medikamentöse und nichtmedikamentöse Maßnahmen entworfen. Nach intensiven Mitarbeiterschulungen wurden diese Konzepte umgesetzt und anschließend nach strengen Kriterien extern evaluiert und auditiert.

Ergebnisse. Es wurden 30 Patienten befragt: $100 \%$ erhielten eine Basis-Schmerzmedikation. In $87 \%$ wurde eine zusätzliche Bedarfsmedikation benötigt. Nur $13 \%$ erlebten die Medikation als zu schwach wirksam, dafür $74 \%$ als wirksam. Die Sternum-Schmerzen waren zu $50 \%$ belastungsinduziert. Dank der intensiven Patienteninformation meldeten sich $100 \%$ der Patienten mit Schmerzen beim Personal, $70 \%$ direkt beim Auftreten.

Schlussfolgerungen. Trotz aller Vorbehalte eines externen Audit scheint nur dieser Weg zu einer nachhaltigen Optimierung zu führen. Entscheidend für einen bleibenden Erfolg werden jedoch die jährlichen,internen Auditierungen sowie die externe Rezertifizeirung in 3 Jahren sein.

\section{7}

Die Induktion einer milden Hypothermie $\left(\mathrm{MH}, 33^{\circ} \mathrm{C}\right)$ verbessert die systemische Sauerstoffbilanz während eines experimentellen ischämischen Herzversagens

M. Schwarzl ${ }^{1}$, P. Steendijk ${ }^{2}$, S. Huber ${ }^{3}$, H. Mächler ${ }^{3}$, M. Truschnig-Wilders ${ }^{4}$, B. Pieske ${ }^{1}$, H. Post $^{1}$

${ }^{1}$ Medizinische Universität Graz, Klin. Abteilung für Kardiologie, Graz, Austria; ${ }^{2}$ Leiden University Medical Center, Leiden, Netherlands; ${ }^{3}$ Medizinische Universität Graz, Klin. Abteilung für Herzchirurgie, Graz, Austria; ${ }^{4}$ Institut für Medizinische und Chemische Labordiagnostik, Medizinische Universität Graz, Graz, Austria

Grundlagen. Die Induktion der MH nach Kreislaufstillstand verringert hypoxische Organschäden. MH verbessert zudem die systolische Funktion des Herzens und reduziert den Gesamtkörper-Sauerstoffbedarf $\left(\mathrm{VO}_{2}\right)$. Wir untersuchten Effekte von MH während akuter Myokard-Ischämie.

Methodik. Bei anästhesierten Schweinen ( $n=14$, $71 \pm 1 \mathrm{~kg}$ ) wurde wiederholt koronare Mikroembolisation (KME) in das CX-Stromgebiet durchgeführt, bis der Cardiac Power Output um $>40 \%$ reduziert war. Danach wurden die Tiere abwechselnd einer normothermen (NT, $\left.38^{\circ} \mathrm{C}\right)$ oder hypothermen Gruppe $\left(\mathrm{MH}, 33^{\circ} \mathrm{C}\right)$ zugewiesen. Ergebnisse entsprechen $6 \mathrm{~h}$ nach KME $v s$. Kontrolle. $*: p<0.05$ vs. Kontrolle, \#: $p<0.05$ vs. NT.

Ergebnisse. Die Herzfrequenz (bpm) stieg während NT $\left(100 \pm 7^{*}\right.$ vs. $\left.88 \pm 4\right)$, fiel jedoch während MH $(65 \pm$ $4^{*, \#}$ vs. $\left.86 \pm 4\right)$. Das Herzzeitvolumen $(\mathrm{l} / \mathrm{min})$ war in beiden Gruppen reduziert (NT: $3.4 \pm 0.3^{*} v s$. $6.1 \pm 0.3$, MH: $3.4 \pm 0.2^{*}$ vs. $6.4 \pm 0.4$ ), aber der aortale Mitteldruck (mmHg) blieb während $\mathrm{MH}$ besser erhalten (MH: $71 \pm 2^{*, \#}$ vs. $106 \pm 3$, NT: $54 \pm 5^{*}$ vs. $\left.106 \pm 5\right) . \mathrm{Da} \mathrm{VO}_{2}$ (ml/min) während $\mathrm{MH}$ stark sank $\left(\mathrm{MH}: 193 \pm 8^{*}, \#\right.$ vs. $332 \pm 18$, NT: $270 \pm 13$ vs. $312 \pm 12$ ), lag die gemischtvenöse Sauerstoffsättigung (\%) während $\mathrm{MH}$ deutlich höher (MH: $65 \pm 3$ vs. $60 \pm 4$, NT: $41 \pm 3^{*, \#}$ vs. $67 \pm 1$ ).

Schlussfolgerungen. $\mathrm{MH}$ verbessert die systemische Sauerstoffbilanz während akut ischämischen Herzversagens durch Senken des Sauerstoffbedarfs. MH könnte daher eine therapeutische Option auch bei akutem Herzversagen und kardiogenem Schock darstellen.

\section{8}

Experimenteller No-Reflow-Infarkt durch koronare Mikroembolisation führt zu akuter, progressiver diastolischer Herzinsuffizienz

M. Schwarzl ${ }^{1}$, P. Steendijk ${ }^{2}$, S. Huber ${ }^{3}$, H. Mächler ${ }^{3}$, M. Truschnig-Wilders ${ }^{4}$, B. Pieske ${ }^{1}$, H. Post ${ }^{1}$ 
${ }^{1}$ Medizinische Universität Graz, Klin. Abteilung für Kardiologie, Graz, Austria; ${ }^{2}$ Leiden University Medical Center, Leiden, Netherlands; ${ }^{3}$ Medizinische Universität Graz, Klin. Abteilung für Herzchirurgie, Graz, Austria; ${ }^{4}$ Medizinische Universität Graz, Institut für Medizinische und Chemische Labordiagnostik, Graz, Austria

Grundlagen. Eine koronare Mikroembolisation (KME) durch rupturierte atherosklerotische Plaques tritt häufig spontan auf und verkompliziert koronare Interventionen. Experimentelle KME des Ramus circumflexus (RCX) beeinträchtigt die regionale Wandverdickung des linken Ventrikels (LV). Wir untersuchten akute Effekte von KME auf die globale LV Funktion.

Methodik. $45 \mu \mathrm{m}$ Polystyrol-Mikrosphären wurden wiederholt in den RCX anästhesierter Schweine $(n=8$, $69 \pm 2 \mathrm{~kg}$ ) injiziert, bis der Cardiac Power Output um $>40 \%$ reduziert war. Ergebnisse entsprechen Kontrolle, unmittelbar (KME 0) und $6 \mathrm{~h}$ nach KME (KME 6). *: $p<0,05$ vs. Kontrolle, \#: $p<0,05$ vs. KME 0 .

Ergebnisse. Die Herzfrequenz stieg von $86 \pm 4$ und 86 \pm 4 auf $99 \pm 6^{*, \#} \mathrm{bpm}$, das Herzzeitvolumen sank von 6,2 $\pm 0,3$ auf $4,2 \pm 0,2^{*}$ und $3,5 \pm 0,3^{*} \mathrm{l} / \mathrm{min}$, der maximale LV Druck von $121 \pm 5$ auf $86 \pm 3^{*}$ und $68 \pm 4^{*, \#} \mathrm{mmHg}$, und die gemischtvenöse Sauerstoffsättigung von $67 \pm 1$

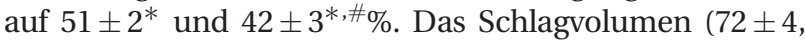
$49 \pm 2^{*}$ und $36 \pm 3^{*, \#} \mathrm{ml}$ ) sowie das LV end-diastolische Volumen $\left(134 \pm 7,95 \pm 4^{*}, 66 \pm 4^{*, \#} \mathrm{ml}\right)$ fielen proportional, während die Ejektionsfraktion unverändert blieb $(52 \pm 2,52 \pm 2,55 \pm 3 \%)$. Die end-diastolische DruckVolumen-Beziehung war progressiv nach links verschoben.

Schlussfolgerungen. In diesem no-reflow InfarktModell kann der LV zunehmend keine adäquate Vorlast aufzunehmen (= diastolische Herzinsuffizienz). Die Ejektionsfraktion spiegelt den massiven LV Funktionsverlust nicht wider. Diastolisches Herzversagen könnte daher bei Patienten mit no-reflow Infarkt häufig unterschätzt sein.

\section{9}

Intravenous and intramyocardial injection of irradiated apoptotic peripheral blood mononuclear cells (PBMC) preserves ventricular function after myocardial infarction

\footnotetext{
M. Lichtenauer ${ }^{1,2}$, M. Mildner ${ }^{3}$, K. Hoetzenecker ${ }^{1,2}$, M. Hasun ${ }^{4}$, A. Baumgartner ${ }^{4}$, G. Werba ${ }^{1,2}$, S. Nickl ${ }^{1,2}$ M. Zimmermann ${ }^{1,2}$, A. Mitterbauer ${ }^{1,2}$, B. K. Podesser ${ }^{4}$, W. Klepetko ${ }^{1}$, H. J. Ankersmit ${ }^{1,2}$

${ }^{1}$ Department of Surgery, Medical University of Vienna, Vienna, Austria; ${ }^{2}$ Christian Doppler Laboratory for Cardiac and Thoracic Diagnosis and Regeneration, Vienna, Austria; ${ }^{3}$ Department of Dermatology, Medical University of Vienna, Vienna, Austria; ${ }^{4}$ Ludwig Boltzmann Cluster for Cardiovascular Research, Vienna, Austria
}

Background. Previous studies have demonstrated that infusion of apoptotic cells lead to an initiation of immunosuppressive mechanisms. Based on these reports, we hypothesized that injection of apoptotic cells into ischaemic myocardium reduces inflammatory reactions after myocardial infarction (MI).

Methods. Cell suspensions of apoptotic cells were injected intravenously (IV) or intramyocardially (IM) in a rat model of MI. Cardiac function was analyzed by echocardiography and infarction size was determined by planimetry after 6 weeks.

Results. Rats that were injected with irradiated apoptotic PBMC showed enhanced homing of macrophages and endothelial progenitor cells (EPC) within 72 hours as compared to controls. Planimetric analysis showed a significant reduction of infarction size and improvement of post MI remodeling with less signs of dilation (infarct dimension 5\% in IV injected animals, 9\% in IM injected rats, $25 \%$ in controls, $p<0.001$ ). Echocardiography revealed that ventricular function was almost preserved in the treatment groups with EF values of $53 \%$ and $55 \% v s$. $42 \%$ in untreated controls compared to $61 \%$ in sham operated rats $(n=13$ per group, $p<0.01$ ).

Conclusions. These data indicate that irradiated apoptotic PBMC suspensions administered IV or IM caused the homing of regenerative EPC and macrophages after $\mathrm{MI}$ and preserved cardiac function.

\section{0}

\section{Administration of anti-thymocyte globulin (ATG) preserves cardiac function after experimental myocardial infarction}

\section{Lichtenauer ${ }^{1,2}$, G. Werba ${ }^{1,2}$, M. Mildner ${ }^{3}$, M. Hasun ${ }^{4}$, A. Baumgartner ${ }^{4}$, S. Nickl ${ }^{1,2}$, M. Zimmermann ${ }^{1,2}$, B. K. Podesser ${ }^{4}$, W. Klepetko', H. J. Ankersmit ${ }^{1,2}$}

${ }^{1}$ Department of Surgery, Medical University of Vienna, Vienna, Austria; ${ }^{2}$ Christian Doppler Laboratory for Cardiac and Thoracic Diagnosis and Regeneration, Vienna, Austria; ${ }^{3}$ Department of Dermatology, Medical University of Vienna, Vienna, Austria; ${ }^{4}$ Ludwig Boltzmann Cluster for Cardiovascular Research, Vienna, Austria

Background. Reports have showed that infusion of apoptotic leucocytes or anti-lymphocyte serum after myocardial infarction (MI) can reduce myocardial necrosis and preserves cardiac function. In order to corroborate this therapeutic mechanism, the utilisation of immunosuppressive agents with a comparable mechanism (anti-thymocyte globulin, ATG) was evaluated.

Methods. MI was induced in rats. Initially after the onset of ischaemia, ATG (10 mg/rat) was injected intravenously. Histological evaluations were performed three days after MI in order to analyze angiogenic cell popula- 
tions in the infarcted myocardium. Cardiac function was analyzed by echocardiography and infarction size was determined by planimetry.

Results. Rats that were injected with ATG evidenced higher numbers of macrophages and endothelial progenitor cells (EPC) in the ischemic myocardium 72 hours after MI as compared to controls. Animals injected with ATG showed a significant reduction of infarct dimension and an improvement of post MI remodelling (infarct dimension $26 \%$ vs. $12.5 \%, p<0.01$ ). Furthermore, echocardiography revealed an improved functional recovery in treated animals as evidenced by a reduced loss of ejection fraction $(43 \%$ in controls vs. $52 \%$ in treated animals, $p<0.01, n=13$ per group).

Conclusions. These data indicate that ATG salvaged ischaemic myocardium, increased the homing of macrophages and EPC and improved cardiac function after experimental MI.

\section{Österreichische Gesellschaft für Kinder- und Jugendchirurgie: Technik, Varia}

\section{1}

\section{Defektverschluss der congenitalen diaphragmatischen Hernie (CDH) mit direkter Naht im Vergleich zum Interponat: Eine multizentrische Studie}

\section{E. M. Ruttenstock ${ }^{1}$, R. Doodnath ${ }^{2}$, J. W. Duess ${ }^{1}$, M. Garriboli ${ }^{3}$, A. Pierro ${ }^{3}$, M. E. Höllwarth ${ }^{1}$, P. Puri ${ }^{2}$}

${ }^{1}$ Abteilung für Kinder - und Jugendchirurgie, Medizinische Universität Graz, Graz, Austria; ${ }^{2}$ National Children's Research Centre, Our Lady's Children's Hospital Crumlin, Dublin, Ireland; ${ }^{3}$ UCL Institute of Child Health, Great Ormond Street Hospital for Children London, London, UK

Grundlagen. Eine kürzlich publizierte Studie suggerierte die Dimension des diaphragmatischen Defekts als bedeutungsvollsten Risikofaktor für die Sterblichkeitsrate bei CDH. Da große Defekte standardgemäß mit Interponaten repariert werden, war Ziel dieser Studie mittels Gegenüberstellung von Defektreparatur mit Interponat zu direkter Naht (DN), Zusammenhang zwischen Defektgröße und Patientenoutcome zu evaluieren.

Methodik. Im Zeitraum von zehn Jahren (2000-2009) wurden die Daten von Patienten mit operierter $\mathrm{CDH}$ in drei kinderchirurgischen Zentren retrospektiv analysiert. Der Fokus dieser Analyse wurde auf demographische Daten, chirurgischen Verfahrensweg, Outcome und Überlebensrate von Patienten mit Interponat gegenüber Patienten mit DN gerichtet.
Ergebnisse. Aus einem Gesamtkollektiv von 208 Patienten erhielten 74 (35,6\%) ein Interponat, 134 $(64,4 \%)$ eine Defektreparatur mit DN. In der Interponatgruppe war eine pränatale Diagnose signifikant häufiger gestellt worden $(64,9 \%$ vs. 20,9\%, $p<0,001)$. Gestationsalter, Geburtsgewicht und Extrakorporale Membranoxygenierung (ECMO) zeigten signifikante Unterschiede zwischen Interponat und DN (38 vs. 39, $p<0,001,2960 \mathrm{~g}$ vs. $3200 \mathrm{~g}, p=0,03,14,2 \%$ vs. $2,7 \%, p<0,001)$. Keine Unterschiede wurden beobachtet bezüglich Zugangsweg (offen $v s$. minimal invasiv, $p>0,05)$, Rezidivrate $(13,5 \%$ vs. $9 \%, p=0,349)$ sowie Überlebensrate $(86,5 \%$ vs. $94 \%$, $p=0,075)$.

Schlussfolgerungen. Patienten mit Interponat hatten häufiger eine pränatale Diagnose und benötigten häufiger ECMO, dennoch konnte kein Zusammenhang zwischen Defektgröße und Defektreparatur und der Überlebensrate von Kindern mit CDH gezeigt werden.

\section{2}

\section{Fokal Noduläre Hyperplasie (FNH) bei einem 12-jährigen Knaben - ein Beitrag zu einem seltenen Krankheitsbild}

\section{J. Hager ${ }^{1}$, M. Rieger ${ }^{2}$, W. Jaschke ${ }^{2}$, A. Klein-Franke ${ }^{3}$, T. Hager ${ }^{4}$, B. Zelger ${ }^{4}$ \\ ${ }^{1}$ Kinderchirurgie Innsbruck, Innsbruck, Austria; ${ }^{2}$ Radiologie Innsbruck, Innsbruck, Austria; ${ }^{3}$ Pädiatrie II Innsbruck, Innsbruck, Austria; ${ }^{4}$ Pathologie Innsbruck, Innsbruck, Austria}

Grundlagen. Eine fokal noduläre Hyperplasie (FNH) ist ein benigner, ätiologisch ungeklärter, hauptsächlich bei Frauen (Kontrazeptiva) zu beobachtender Lebertumor. Bei Kindern kommt diese Neoplasie selten vor, nur etwa $1-2 \%$ der an sich schon raren Lebertumoren sind FNHs.

Methodik. Ein 12-jähriger Patient, der ein Pfeiffer'sches Drüsenfieber mit Leberbeteiligung durchgemacht hatte, wurde 3 Monate später wegen eines im Zuge einer Abdomen-Kontrollsonographie gefundenen, mannsfaustgroßen Lebertumors (Segment 2/3) zugewiesen. Die bildgebenden Untersuchungen zeitigten einen hochvaskularisierten Tumor, suspekt für eine $\mathrm{FNH}$, ein Leberzellkarzinom war nicht auszuschließen. Auf Grund seiner ausgeprägten Vaskularisation wurde auf eine Feinnadelbiopsie verzichtet. Präoperativ wurde eine Tumurgefäßembolisation vorgenommen. Die Tumorresektion, der Tumor tangierte die linke Lebervene, gestaltete sich problemlos.

Ergebnisse. Histopathologisch zeigte sich eine nodulär strukturierte, aus differenzierten, trabekulär angeordneten Hepatozyten und Gallenwegsproliferationen aufgebaute FNH. Der postoperative Verlauf war bislang, wie die seit 3 Jahren jährlich durchgeführten Verlaufskontrollen ergaben, komplikationslos.

Schlussfolgerungen. Eine FNH gilt bei Kindern, abgesehen von „Langzeitüberlebenden“ nach Radio-Chemotherapie, als seltene Entität. Eine sichere Diagnostik ist oft 
nicht einfach, bzgl. einer Tumorbiopsie gibt es konträre Meinungen. Eine Resektion einer FNH ist gewöhnlich nicht notwendig, Ausnahmen sind sehr große oder hinsichtlich. ihrer Zuordnung nicht zu bewertende Tumoren (in einem solchen Fall ist zum Ausschluss eines Malignoms eine histologische Untersuchung unerläßlich).

\section{3}

\section{Management of blunt liver and spleen injuries in children}

\section{O. Renz, B. Häussler, P. Hechenleitner, J. Hager}

Kinderchirurgie Innsbruck, Innsbruck, Austria

Background. An injured parenchymal organ can be preserved either with reparative surgery or observation. This retrospective study bases on 20 years of experience and is designed to analyze the development of different methods of treatment of children.

Methods. To demonstrate the change in treatment, we created two groups: The first group, including 77 patients, was treated between 1990 and 1999. The second group, including 85 patients, was treated between 2000 and 2009. We compared the grade of injury, the outcome according to the type of treatment and the period of hospitalization.

Results. In the first group surgical treatment was performed in 42 of 77 patients i.e. $54 \%$ consisting of 18 liver and 24 spleen injuries. The average stay in hospital was 15 days. In the second group surgical treatment was performed in 20 of 85 patients i.e. $23.5 \%$ consisting of 5 liver and 15 spleen injuries. The average stay in hospital was 12 days.

Conclusions. Due to improved radiological diagnostics and precise clinical monitoring it is nowadays possible to verify more exactly the type of liver and spleen rupture and thus replace surgery by observation.

\section{4}

\section{Zystische Duplikaturen des} Gastrointestinaltraktes: ein Überblick über die Erfahrungen der letzten 10 Jahre an der Abteilung für Kinder- und Jugendchirurgie in Innsbruck

\section{A. Perathoner ${ }^{1}$, I. Gaßner ${ }^{2}$, T. Hager ${ }^{3}$, B. Häussler ${ }^{1}$, P. Hechenleitner ${ }^{1}$, J. Hager ${ }^{1}$ \\ ${ }^{1}$ Kinderchirurgie Innsbruck, Innsbruck, Austria; \\ ${ }^{2}$ Universitätsklinik f. Pädiatrie, Innsbruck, Austria; ${ }^{3}$ Institut für Pathologie Innsbruck, Innsbruck, Austria}

Grundlagen. Zystische Duplikaturen des Gastrointestinaltraktes sind rare kongenitale Fehlbildungen (0,1-
0,3\% aller intestinalen Anomalien), die im gesamten Verdauungskanal vorkommen können, zumeist symptomlos sind und selten mechanische (Darmkompression) oder gewebeassoziierte (Blutung) Komplikationen bedingen. Sie werden typischerweise bei Feten im Rahmen der sonographischen Schwangerschaftsüberwachung oder zufällig bei der Abklärung abdomineller Beschwerden anderer Provenienz gefunden. Die Behandlung besteht in einer Zystenenukleation oder einer Resektion des betroffenen Darmsegments.

Methodik. Während der letzten 10 Jahre wurden bei 11 Kindern (6 Mädchen und 5 Knaben, Alter zwischen 5 Tage und 4 Jahre) insgesamt 13 intestinale Duplikaturen diagnostiziert. 2 betrafen den Oesophagus (verfiziert bei Refluxabklärung), eine das Duodenum, $5(+1)$ das Ileum, $3(+1)$ den Ileozoekalbereich. Abgesehen von den beiden Kindern mit Oesophagusduplikatur wurden alle anderen operiert (2 wegen eines duplikaturbedingten Ileus): Bei zweien wurde die Duplikatur (Duodenum, Zoekum) enukleiert, bei 7 das betroffene Darmsegment (Ileum (3), Ileum/Zoekum (4)) reseziert.

Ergebnisse. Der postoperative Verlauf war bei allen Kindern komplikationslos. Die beiden Kinder mit den Oesophagusduplikaturen sind - Beobachtungszeitraum von 2 bzw. 4 Jahren - nach wie vor beschwerdefrei.

Schlussfolgerungen. Da zystische Duplikaturen des Gastrointestinaltraktes durch Größenzunahme oder ektope Schleimhautareale ein gewisses Komplikationsrisiko (Kompressionsileus, Invagination, Zystenblutung) haben, sollte auch bei asymptomatischem Zufallsbefund eine frühelektive chirurgische Therapie angestrebt werden.

\section{5}

\section{Riesiges Lipom des Retroperitoneum - eine seltene Ursache für eine Beeinträchtigung der Darmpassage bei einem 12-jährigen Knaben}

\section{J. Hager ${ }^{1}$, P. Hechenleitner' ${ }^{1}$, I. Gaßner ${ }^{2}$, T. Hager ${ }^{3}$, C. Ensinger ${ }^{3}$ \\ ${ }^{1}$ Kinderchirurgie Innsbruck, Innsbruck, Austria; ${ }^{2}$ Radiologie der Univ.-Klinik f. Kinder- u. Jugendheilkunde Innsbruck, Innsbruck, Austria; ${ }^{3}$ Institut für Pathologie Innsbruck, Innsbruck, Austria}

Grundlagen. Fettgewebsgeschwülste des Retroperitoneum kommen bei Kindern selten vor und werden erst nach Erreichen einer beträchtlichen Größe symptomatisch. Zur Globaldiagnose „Lipom“ reicht die Standardbildgebung (Abdomen-Sonographie bzw. -MRT). Therapeutisch ist eine R-0-Resektion anzustreben. Wegen der sich biologisch unterschiedlich verhaltenden Fettgewebsgeschwülste (reifes Lipom, Lipoblastom, Lymphangiolipom - benigne, Liposarkom maligne) ist eine pathohistologische Untersuchung unerläßlich. 
Methodik. Bei einem 12-jähriger Knaben mit seit Wochen bestehenden Bauchschmerzen und Änderung der Stuhlgewohnheiten ergab die Abdomen-Sonographie als Geschehensursache den Verdacht auf ein retroperitoneales Lipom (Durchmesser $12 \mathrm{~cm}$ ), „bestätigt“ durch eine Doppler-Sonographie bzw. die für diesen Tumor typische Avaskularität und durch ein MRT des Abdomens. Bei der Operation fand sich der Prozess im Ileozökalwinkel, terminales Ileum, Zökum und Colon ascendens deutlich imprimierend. Er wurde radikal unter Erhalt des ileokolischen und rechtskolischen Gefäßbündels reseziert.

Ergebnisse. Der postoperative Verlauf gestaltete sich problemlos. Pathohistologisch wurde der Tumor als reifes, in sano reseziertes Lipom eingestuft.

Schlussfolgerungen. Bei Kindern gelten vom Fettgewebe des Retroperitoneum ausgehende Tumoren als selten; mittels Standardbildgebung sie sind relativ einfach als „Lipome“ zu verifizieren. Obwohl nur myxoide Lipoblastome im Langzeitverlauf eine maligne Entartung zeigen und Liposarkome äußerst selten vorkommen, ist wegen der intraoperativ nicht sicher einzustufenden Tumorentität und wegen des Rezidivrisiko eine radikale Tumorresektion notwendig.

\section{6}

\section{Infiziertes retroperitoneales zystisches Lymphangiom: Eine diagnostische, therapeutische und histopathologische Herausforderung}

\author{
J. Hager ${ }^{1}$, B. Häussler ${ }^{1}$, R. Kafka-Ritsch ${ }^{1}$, I. Gaßner ${ }^{2}$, \\ T. Hager ${ }^{3}$, G. Mikuz ${ }^{3}$ \\ ${ }^{1}$ Kinderchirurgie Innsbruck, Innsbruck, Austria; ${ }^{2}$ Radiologie \\ der Universitätsklinik für Kinder- und Jugendheilkunde, \\ Innsbruck, Austria; ${ }^{3}$ Institut für Pathologie, Innsbruck, Austria
}

Grundlagen. Retroperitoneale zystische Lymphangiome sind selten, das Gros wird im Kindesalter symptomatisch. Im Falle einer Infektion sind sie eine Herausforderung, zumal die Diagnostik schwierig und die Therapie - Tumordrainage oder Tumorexzision komplikationsbelastet ist.

Methodik. Bei 2 männlichen Patienten (9 Monate, 16 Jahre) wurden auswärts retroperitoneale Tumoren (Durchmesser 10 bzw. $12 \mathrm{~cm}$ ) festgestellt, die nach CTUntersuchung als zystische Lymphangiome eingestuft wurden. Beim jüngeren (hochfiebernden) Kind handelte es sich um einen infizierten Prozess, er wurde unter antibiotischer Behandlung Pigtail-drainiert. Beim älteren Patienten, ohne Anhalt für Tumorinfektion, wurde der intraoperativ nur mehr $6 \mathrm{~cm}$ große, dickwandige und malignomverdächtige (Schnellschnitt!) Tumor „radikal“ reseziert.

Ergebnisse. Wegen der beim Säugling ineffizienten Behandlung (Bacteroides-fragilis-Infektion) wurde der
Prozess nach Antibiotikumwechsel unter Kapselerhaltung ausgeräumt. Er bildete sich rasch zurück und war 6 Wochen später nicht mehr nachzuweisen. Beim älteren Patienten stellte sich postoperativ eine massive, nach16 Tagen spontan sistierende Lymphsekretion ein. Histopathologisch zeigten beide Operationspräparate nur Abszessformationen und Granulationsgewebe, aber kein zystischlymphangiomatöses Gewebe.

Schlussfolgerungen. Die Ursache für Infektionen retroperitonealer zystischer Lymphangiome ist unklar, möglicherweise aber auf Enteritiden zurückzuführen. Die bildgebende Diagnostik ist schwierig, das therapeutische Vorgehen kann Probleme machen. Die histopathologische Untersuchung bleibt unbefriedigend, zumal nur eine Gewebsbeurteilung im Sinne von nicht-maligne $v s$. maligne möglich ist.

\section{7}

Regelmässige Ultraschalluntersuchungen bei Verdacht auf Appendicitis reduzieren die Aufnahme- und Operationszahlen im Kindesalter

\section{E. Q. Haxhija, A. S. Komoni, D. Bäumel, U. Seebacher, M. Höllwarth}

Universitätsklinik für Kinder- und Jugendchirurgie, Medizinische Universität Graz, Graz, Austria

Grundlagen. Diese retrospektive Studie untersucht den Zusammenhang zwischen der stark angestiegenen Anzahl der Ultraschalluntersuchungen zur Diagnostik der Appendizitis und der Anzahl der Kinder und Jugendlichen, die im selben Zeitraum (1994-2008) aufgrund von Appendizitis entweder stationär aufgenommen oder operiert wurden.

Methodik. Alle Ultraschallbefunde wurden bezüglich der Appendixerkennungsrate analysiert. Alle pathohistologischen Befunde wurden zur Bestimmung der Art der Appendizitis ausgewertet. Patienten wurden in 4 Gruppen eingeteilt: 1) perforierte Appendizitis; 2) eitrig-gangrenöse Appendizitis; 3) einfache Appendizitis (alle andere operierte Patienten); und 4) stationär beobachtete, aber nicht operierte Patienten. Für die statistische Analyse wurde Spearman's Korrelationskoeffizient herangezogen.

Ergebnisse. Der kontinuierliche Anstieg der abdominellen Ultraschalluntersuchungen zeigte sich von einer kontinuierlich steigenden Appendixerkennungsrate begleitet $(p<0,001)$. Zugleich wurde ein signifikanter Abfall der Anzahl der wegen Appendizitis sowohl stationär beobachteten, als auch operierten Patienten festgestellt $(p<0,001)$. Der signifikante Abfall der Appendektomierate betraf lediglich die Gruppe der Patienten mit einfacher Appendizitis $(p<0,001)$, während keine signifikante Änderungen in der Anzahl der operierten Patienten mit eitriger oder perforierter Appendizitis zu finden waren. 
Schlussfolgerungen. Regelmäßige Ultraschalluntersuchungen der Kinder und Jugendlichen mit Verdacht auf Appendizitis sind $\mathrm{zu}$ empfehlen, da diese sowohl zur signifikanten Reduktion der Gesamtzahl der stationär aufgenommen Patienten, als auch zur Reduktion der wegen einfacher Appendizitis operierten Patienten führen.

\section{8}

\section{Gastrostomie und Jejunostomie zur frühzeitigen enteralen Ernährung bei Kindern und Jugendlichen mit schwerem Schädel-Hirn-Trauma}

\section{J. Schalamon, P. A. Hausbrandt, P. H. Schober,}

\section{A. K. Saxena, M. E. Höllwarth}

Universitätsklinik für Kinder- und Jugendchirurgie Graz, Graz, Austria

Grundlagen. Wir analysierten eine Serie von Kindern und Jugendlichen mit schwerem Schädel-Hirn Trauma (SHT), die frühzeitig mit einer offenen Gastrostomie und Jejunostomie zur enteralen Ernährung versorgt wurden.

Methodik. Wir inkludierten alle Kinder und Jugendlichen mit schwerem Schädel-Hirn Trauma $(\mathrm{GCS}<9)$, die zwischen Januar 2005 und März 2010 mit Gastrostomie und Jejunostomie versorgt wurden. Der Unfallmechanismus, Begleitverletzungen, Auftreten einer Pneumonie, Beatmungsdauer, Krankenhausaufenthalt, Sonden-Komplikationen und Glasgow Outcome Score (GOS) wurden dokumentiert.

Ergebnisse. Im Studienzeitraum wurden 31 Patienten ( $22 \mathrm{~m}, 9$ w, Alter: 1-17 Jahre, $\varnothing 11$ Jahre) inkludiert. Offene Gastrostomie und Jejunostomie wurden meist bereits am Aufnahmstag angelegt $(n=16)$, bei 15 weiteren Patienten erfolgte die Sondenimplantation 1-10 Tage (median 2 Tage) nach initialem Trauma. Bei 5 Kindern (16\%) traten Komplikationen auf, die schwerwiegendste war ein Volvolus um die Jejunalsonden-Einbringungsstelle. Bei 6 Kindern wurde eine Pneumonie diagnostiziert, meist als Folge einer Aspiration am Unfallort. Die mediane Beatmungsdauer betrug 8 Tage, die Aufenthaltsdauer 28 Tage, der GOS war 4

Schlussfolgerungen. Durch die Anlage einer Gastrostomie und Jejunostomie wird die posttraumatische Magenentleerungsstörung umgangen und die Rate an Ventilator-assoziierten Pneumonien niedrig gehalten. Die frühzeitige Anlage einer Gastrostomie und Jejunostomie zur enteralen Ernährung bei pädiatrischen Patienten mit schwerem SHT hat sich als sicher und komplikationsarm erwiesen.

\section{Hauptsitzung - Chirurgie und} Recht

\section{4}

\section{Anforderungen an ein medizinisches Gutachten}

\section{H. Diemath}

Gerichtssachverständiger, Salzburg, Austria

Bewährter Aufbau eines Gutachtens:

Fragestellung und Aktenlage; Vorgeschichte; Auszug aus den Krankengeschichten; Vorgutachten; Angaben über derzeitige Beschwerden; Untersuchungsbefund; Krankheitsbezeichnung; Beurteilung, Auseinandersetzung mit den Vorbefunden und Vorgutachten, wissenschaftliche Begründung; Schrifttum; Zusammenfassung und Begutachtung.

Die Persönlichkeitsrechte sind $\mathrm{zu}$ beachten (ordnungsgemäße Aufklärung). Diese hat alle Bereiche zu umfassen: Diagnose, natürlichen Verlauf der Erkrankung, Behandlung, Behandlungsalternativen, Risiko, Verfahren der Unterlassung/Diagnose, Behandlung, Nachbehandlung. Rund $90 \%$ aller Haftpflichtprozesse werden durch eine fehlende oder mangelhafte Aufklärung begründet.

Die vollständigen Krankengeschichten sind unabdingbar. Die Ausfolgung muss erfolgen, sonst $\S 295$ StGB, „Unterdrückung von Beweismitteln“ (Freiheitsstrafen bis zu einem Jahr).

Alle Vorgutachten sind anzufordern und zu beachten.

Die „derzeitigen Beschwerden“ sollten immer in der Ich-Form wiedergegeben werden (wichtig: Angabe der derzeitigen Heilmitteleinnahme).

Verdeutlichungstendenzen sind festzuhalten.

Für die Duldungspflicht (Mitwirkungspflicht, Schaden, Minderungspflicht), gilt die Zumutbarkeit (OGHUrteile). Die Eingriffe müssen einfach, gefahrlos, ohne nennenswerte Schmerzen, mit sicherer Aussicht auf Erfolg sein. Die Duldung entscheidet immer der Patient.

Die Beurteilung der Einlassungsfahrlässigkeit ist besonders schwierig; das "ante hoc“ ist entscheidend.

Die Angabe von Schrifttum ist zweckmäßig (fachliche Kompetenz).

Von gutachterlicher Belange sind in der ärztlichen Ausbildungsordnung vorgesehen. Ärztliche Gutachten sind für die Rechtssprechung entscheidend und unverzichtbar. Damit erfüllen sie auch einen gesellschaftspolitischen Auftrag. 


\section{Österreichische Gesellschaft \\ für Handchirurgie: Toxische Schädigungen an Hand und Unterarm}

\section{7}

\section{Freier Gewebetransfer bei einem acht Monate alten Säugling nach Paravasation von Arterenol ${ }^{\circledR}$ im Rahmen einer Meningokokkensepsis}

\author{
M. Hladik', G. Schimpl ${ }^{2}$, W. Radauer ${ }^{3}$, H. Schubert ${ }^{1}$, \\ F. Ensat ${ }^{1}$, E. Russe ${ }^{1}$, P. Pülzl ${ }^{1}$, G. Wechselberger ${ }^{1}$, \\ M. Hladik ${ }^{1}$
}

\begin{abstract}
${ }^{1}$ Abteilung für Plastische und Wiederherstellungschirurgie, Krankenhaus der Barmherzigen Brüder Sbg, Salzburg, Austria; ${ }^{2}$ Universitätsklinik für Kinder und Jugendchirurgie, Salzburg, Austria; ${ }^{3}$ Universitätsklinik für Kinder und Jugendheilkunde, Salzburg, Austria
\end{abstract}

Der freie Gewebetransfer ist mittlerweile nicht nur bei Erwachsenen sondern auch bei Kindern ein etabliertes Verfahren, welches jedoch bei Kleinkindern und Säuglingen nur selten zur Anwendung kommt. Wir berichten über einen 8 Monate alten Buben der einen septischen Schock im Rahmen einer Menigokokkeninfektion entwickelte. Aufgrund der schlechten Kreislaufsituation wurden initial transossäre Zugänge gelegt, wobei es zu einer Paravasation von Noradrenalin (Arterenol ${ }^{\circledR}$ ) im Bereich des linken Knies kam. Nach Nekrosektomie zeigte sich ein ca. $7 \times 6 \mathrm{~cm}$ großer Defekt mit freiliegenden Sehnen und Knochen, weshalb wir uns zur Defektdeckung mittels freiem myokutanem Latisssimus dorsi Lappen entschieden. Die mikrochirurgische Anastomosierung erfolgte End-zu-End an die A. und V. tibialis posterior. Der peri- und postoperative Verlauf waren komplikationslos. Letztendlich erholte sich der Säugling von der Infektion und sowohl eine vollständige Belastbarkeit als auch ein uneingeschränkter Bewegungsumfang des Beins konnten erzielt werden. Mit der Darstellung dieses seltenen Behandlungsverlaufes wollen wir das Bewusstsein schaffen, dass auch bei Säuglingen nicht vor einer derart komplexen Versorgung zurückgeschreckt werden sollte.

\section{8}

Die Indocyaningrün- Angiographie zur Darstellung der Perfusion bei mikrovaskulären Transplantaten nach Tumor- und Osteoradionekroseresektionen

L. Gerzanic ${ }^{1}$, H. Kärcher ${ }^{1}$, G. Zöch ${ }^{2}$
${ }^{1}$ Klinische Abteilung für Mund-, Kiefer- und Gesichtschirurgie, Graz, Austria; ${ }^{2}$ Plastische Chirurgie, SMZ-Ost Donauspital, Wien, Austria

Grundlagen. Die Spenderregion für ein mikrovaskuläres Transplantat muss entsprechend der Dimension des knöchernen und/oder Weichteildefektes gewählt werden. Eine suffiziente Anastomosierung der Transplantatgefäße und Halsgefäße um eine sofortige Transplantatdurchblutung und Revitalisierung zu ermöglichen, kann durch den Farbstoff Indocyaningrün sichtbar gemacht werden.

Methodik. Mit Hilfe der Video- Fluoreszenz- Angiographie und Infrarot-PDE-Kamera wird der Durchblutungszustand und die Nekrosenflächen während und nach der Operation visualisiert, nachdem Indocyaningrün über die Vene injiziert wurde.

Ergebnisse. Bei 3 Tumorpatienten und 3 Osteoradionekrosepatienten ist Indocyaningrün injiziert worden und nach der Anastomisierung des mikrovaskulär angeschlossenen Transplatates die Durchblutung überprüft und dargestellt worden. Auch ein Verlust des Transplantates durch Nekrose kann mit Indocyanin durch eine Laseroder Infrarat- Kamera dargestellt werden. Auf der Intensivstation ist es möglich mit Indocyaningrün die Vitalität und Perfusion des Transplantates unmittelbar nach der Operation bei geringer Abdunkelung sichtbar zu machen.

Schlussfolgerungen. Es wird anhand von Fallbeispielen eine Darstellung und Perfusion des Transplantates unmittelbar nach der Anastomosierung und postoperativ in Videos dargestellt. Eine Weichteil-Perfusionsszintigraphie kann ein solches Verfahren am Patientenbett unmittelbar nach der Operation nicht ersätzen, jedoch bestätigen und unterstützen. Neben dem PET/CT und der Knochenszintigrahie ist die ICG-Angiographie eine neue Methode, die Perfusion eines mikrovaskulären Transplantates während und nach der Operation bei Tumoren und Osteoradionekrosen zu unterstützen.

\section{Österreichische Gesellschaft für Handchirurgie: Verbrennungen an Hand und Unterarm}

\section{9}

\section{Die Behandlung von Handverbrennungen: Ein Update}

\author{
L. Kamolz ${ }^{1,2}$ \\ ${ }^{1}$ Medizinische Universität Wien, AKH Wien; Abteilung \\ für Plastische und Rekonstruktive Chirurgie, Wien, Austria; \\ ${ }^{2}$ LK Wr. Neustadt/Plastische, Ästhetische und Rekonstruktive \\ Chirurgie, Wr. Neustadt, Austria
}

In mehr als $80 \%$ der Brandverletzungen sind die Hände beteiligt. Selbst wenn eine verbrannte Hand nicht 
die Hauptrolle für das Überleben eines Patienten spielt, so ist die Wiederherstellung der Handfunktion für den Patienten von äußerster Wichtigkeit. Die wiederhergestellte Handfunktion ist nämlich für die erfolgreiche Reintegration in die Gesellschaft und das Berufsleben von entscheidender Bedeutung.

Ziel dieses Vortrages ist einen Überblick über die Behandlung von Handverbrennungen zu liefern. Hauptaugenmerk soll dabei auf die Schlüsselfaktoren gelegt werden.

Die Behandlung von Handverbrennungen basiert auf folgende Faktoren:

- Entscheidung bezüglich der Notwendigkeit einer Escharotomie in der frühen posttraumatischen Phase

- Tiefenspezifische Behandlung in der Frühphase

- Timing der Operationen

- Tiefenspezifischen Deckung der Verbrennungswunde

- Frühzeitige Mobilisierung und Rehabilitation

- Regelmäßige Kontrollen und intensive Nachbehandlung inklusive Korrekturoperationen.

Auch wenn wie bereits gesagt eine verbrannte Hand nicht für das Überleben eines Patienten entscheidend ist, so ist die Wiederherstellung der Handfunktion für den Patienten von äußerster Wichtigkeit. Der Patient profitiert daher von der interdisziplinären Zusammenarbeit von mit Brandverletzungen erfahrenen Chirurgen, Physiotherapeuten, Psychologen und Pflege.

\section{1}

Der radial gestielte, fasciocutane VY-Vorschublappen: eine Alternative in der Defektdeckung am Ellbogen

\section{W. Michlits ${ }^{1}$, C. Windhofer ${ }^{2}$, C. Papp ${ }^{3}$}

${ }^{1}$ KA Rudolfstiftung, Abteilung für Plastische, Ästhetische und Rekonstruktive Chirurgie, Wien, Austria;

${ }^{2}$ Unfallkrankenhaus Salzburg, Salzburg, Austria;

${ }^{3}$ Medicent Salzburg, Salzburg, Austria

Grundlagen. Defekte im Bereich der Ellbogenstreckseite sind sehr häufig. Meist handelt es sich um Läsionen im Rahmen einer Bursitis, um Wundheilungsstörungen nach Operationen oder aber um Defekte aufgrund eines Traumas. Meist sind diese Defekte schwierig zu behandeln, zumal es sich wegen des großen Bewegungsausmaßes um eine funktionell sehr beanspruchte Region handelt, die häufig zu Rezidiven führt.

Methodik. Seit 2005 wurde der radial gestielte, fasciocutane VY-Vorschublappen in 22 Fällen eingesetzt (3 Frauen, 19 Männer, Alter $\emptyset 57$ Jahre). Die Patienten hatten durchschnittlich 2,3 Voroperationen und eine Defektgröße von durchschnittlich $23 \mathrm{~cm}^{2}$.

Ergebnisse. Der postoperative Verlauf war in 18 Fällen völlig komplikationslos. In drei Fällen musste eine Revision erfolgen $(1 \times$ Hämatom, $2 \times$ kleine Wundheilungsstörung); in einem weiteren Fall kam es zu einer kleinen Wundheilungsstörung, die jedoch konservativ abheilte.
Nach einer Ruhigstellung von 2-3 Wochen waren alle Patienten voll belastbar und im Ellbogengelenk uneingeschränkt mobil. In unserer Serie war kein Rezidiv zu verzeichnen.

Schlussfolgerungen. Aufgrund der Lokalisation und der bekannten Problematik rund um Wunden im Bereich des Ellbogens ist die Möglichkeit an Korrekturen limitiert. Unsere Erfahrungen mit dem radial gestielten, fasciocutanen VY-Vorschublappen zeigen sehr eindrucksvoll, dass sich dieser Lappen exzellent für die Deckung von Defekten im Ellbogenbereich eignet und für eine dauerhafte Heilung sorgt.

\section{Arbeitsgemeinschaft für Minimal Invasive Chirurgie: Videositzung}

\section{0}

\section{Single Inzision laparoskopische Cholezystektomie mit dem Spider System}

\section{A. Shamiyeh}

Linzer Ausbildungs- und Forschungsinstitut für Chirurgie und Onkologie und Ludwig Boltzmann Institute for operative laparoscopy, Linz, Austria

Grundlagen. Minimierung des Zugangstraumas, die Reduktion postoperativer Schmerzen sind ein wesentliches Anliegen der Chirurgie. Die Single Inzision Chirurgie soll diesem Ziel dienen und vereint Vorteile der Notes Technik mit der Sicherheit einer konventionellen Laparoskopie. Nachteile der konventionellen SIL Technik sind die unflexiblen teils starren, wenig rotierbaren Instrumente. Mit dem „Spider“ wurde eine Plattform geschaffen, die es ermöglicht über eine einzige Inzision sicher zu operieren. Ein überkreuztes operieren ist nicht mehr erforderlich. Als eine der ersten Abteilungen in Europa war es uns möglich mit diesem System im AKh Linz zu operieren.

Methodik. Vom 6.-10. Dezember 2010 wurden 5 Patienten ( $3 \mathrm{w}, 2 \mathrm{~m}$; mittleres Alter 49,6 [19-67], BMI m27 [20-33] mittels Spider laparoskopisch cholecystektomiert. Operationen erfolgten über einen offenen intraumbilicalen Zugang, die OP Schritte an der Gallenblase waren ident einer standard LC. Alle Operationen wurden exakt dokumentiert und aufgezeichnet.

Ergebnisse. Einmal wurde epigastrisch ein $5 \mathrm{~mm}$ Trokar zum Einbringen eines Klippers gesetzt. $1 \times$ wurde die Exposition durch eine zusätzliche 2,3 mm Stichfasszange verbessert. Der postoperative Verlauf war komplikationslos, die Entlassung zwischen 3-5. Tag (m 4,2).

Schlussfolgerungen. Die technisch noch nicht ganz ausgereiften Instrumente ermöglichen eine Bewegungsfreiheit am Organ, wie dies derzeit kein anderes SIL Sys- 
tem schafft. Persönlich halte ich Entwicklungen dieser Art für den Anfang der Zukunft der Laparoskopie.

\section{1}

\section{SPIDER ${ }^{\mathrm{TM}}$ cholecystectomy}

\section{K. Pimpl, W. Brunner, J. Schirnhofer, C. Mittermair, C. Obrist, H. Weiss}

Chirurgie, Salzburg, Austria

This video demonstrates the first European application of the SPIDER ${ }^{\mathrm{TM}}$ platform during a transumbilical single incision laparoscopic cholecystectomy. The SPIDER's unique design accommodates a range of small flexible instruments plus a $5 \mathrm{~mm}$ laparoscopic camera, all of which are operated through a slim tube. Unlike alternate single port techniques, SPIDER ${ }^{\mathrm{TM}}$ eliminates the awkward crossed arms movement, allowing a single surgeon to operate the device more naturally for true right and left instrument manipulation. This device has the potential of a surgical NOTES platform.

\section{2}

SIL (Single Incision Laparoscopic) Cholezystektomie bei einer tückischen „Normvariante“ der Lebergefäße

\author{
E. Laimer ${ }^{1}$, A. Perathoner ${ }^{1}$, G. Mühlmann ${ }^{1}$, M. Biebl ${ }^{1}$, \\ A. Klaus ${ }^{2}$, J. Pratschke ${ }^{1}$, R. Mittermair ${ }^{1}$ \\ ${ }^{1}$ Univ. Klinik für Viszeral-, Transplantations- und \\ Thoraxchirurgie, Innsbruck, Austria; ${ }^{2}$ Krankenhaus \\ Barmherzige Schwestern, Innsbruck, Austria
}

Grundlagen. Die SIL - Cholezystektomie zählt an unserer chirurgischen Abteilung mittlerweile zur Standardoperation bei akuten und chronischen Erkrankungen der Gallenblase.

Methodik. Die SIL - Cholezystektomie wird an unserer Abteilung über eine $5 \mathrm{~mm}$ Optik mittels einer gebogenen Klemme sowie einem Stromhäckchen routinemäßig durchgeführt. Haltenähte werden nur bei unzureichender Übersichtlichkeit platziert. Neben einem sehr kurzen Ductus cysticus stellen die zahlreichen Verlaufsformen der rechten Leberarterie eine besondere chirurgische Herausforderung dar.

Ergebnisse. In unserem Video präsentieren wir eine SIL - Cholezystektomie eines 34-jährigen Patienten bei Zustand nach lithogener Pankreatitis mit Steinabgang und multiplen Gallenblasensteinen. In unserem Fall präsentierte sich eine aberrierende Leberarterie unmittelbar dorsal des Ductus cysticus. Wir zeigen, dass eine genaueste Präparation mit einer kompromisslosen Darstellung der anatomischen Strukturen zwingend notwendig ist, um schwerwiegende Komplikationen vermeiden $\mathrm{zu}$ können.

\section{3}

\section{Die SILS Ileocoecalresektion}

\section{Haunold, A. Klaus, U. Kozuchowska}

KH der Barmherzigen Schwestern Wien, Wien, Austria

Anhand von 3 konsekutiven Fällen an Ileocoecalresektionen in SILS Technik zeigt das Video unser Vorgehen, wie gut und sicher dieses Verfahren machbar ist und wie ansprechend das kosmetische Ergebnis ist.

Es handelte sich jeweils um endoskopisch nicht entfernbare Läsionen, weil zu flach, zu groß bzw. mit teilweise non lifting sign.

Die laparaskopische Resektion erfolgt über den umbilicalen Nabelport, Colon und Dünndarm können hier, wenn ausreichend mobilisiert, vor die Bauchdecke gezogen werden. Die Anastomose erfolgt händisch oder funktionell Seit zu Seit mit dem geraden Ghia. Eine Drainage ist nicht obligat.

\section{4}

\section{Single port laparoscopic pancreas resections: trouble shooting}

\author{
C. Mittermair, W. Brunner, J. Schirnhofer, K. Pimpl, \\ C. Obrist, H. Weiss \\ Chirurgie, Salzburg, Austria
}

We present a sequence of the first transumbilical single incision laparoscopic pancreas procedures performed at our Department. In five patients tumor resection was carried out following the technical principles of tumor enucleation ( 2 patients), spleen preserving pancreatic tail resection (2 patients) and oncologic en-bloc resection of the pancreatic tail, spleen and corresponding lymph nodes. Tricks and pit-falls are demonstrated.

\section{5}

Initial experience with single incision laparoscopic gastric bypass or sleeve resection

W. Brunner, J. Schirnhofer, R. Frass, N. Waldstein, K. Pimpl, C. Mittermair, C. Obrist, H. Weiss

KH Barmherzige Brüder, Salzburg, Austria 
Background. Single-access laparoscopic surgery should offer minimal scarring without compromising surgical outcome. Herein we describe our initial series of single port laparoscopic gastric bypass or sleeve resection.

Methods. Between 04-2010 and 12-2010 four primary bariatric procedures (3 sleeve gastrectomies, 1 RY-gastricbypass) were performed out of a total number of 896 patients undergoing single access laparoscopic surgery (Mean age 53 years, BMI $43 \mathrm{~kg} / \mathrm{m}^{2}$, sex $4 \mathrm{f}$ ). The entire operation was carried out transumbilically (3) or via an epigastric scar hernia (1) in the French position using a single port system.

Results. All sleeves could be completed without adding an additional trocar. Adhesions were found in 2 patients. Specimen retrieval and stapling was carried out transumbilically. Operation time lasted 90-183 min. There were no intraoperative adverse event. Patients started mobilization at the day of surgery and resumed oral intake after nomal test for leakage. The patient with the scar hernia developed mild wound infection which was treated conservatively. Sufficient weight loss was observed in the follow-up.

Conclusions. Singe port laparoscopic sleeve resection and gastric bypass is feasible and can be carried out via transumbilical approach. It reduces the surgical trauma and allows optimal postoperative cosmesis.

\section{6}

\section{Laparoscopic spleen-preserving resection of the pancreatic tail}

\section{Obrist, W. Brunner, J. Schirnhofer, K. Pimpl, C. Mittermair, H. Weiss}

Chirurgie, Salzburg, Austria

We demonstrate a laparoscopic video of spleen-preserving pancreas resection in a young female patient suffering from a glucagonoma in the tail of the pancreas. Since the tumor could not be visualized intraoperatively, laparoscopic ultrasound was carried out to define the resection plane. According to the technical principles of spleen-preserving pancreas resection, splenic artery and vein could be isolated. The pancreatic body was transected utilizing a stapler. Although laparoscopic pancreas resection have been performed successfully during the last decade wide acceptance is lacking.

\section{7}

\section{Laparoscopic mesh augmented repair of a (giant) Morgagni-Larrey hernia}

\section{S. A. Bischofberger, N. Kalak, A. Zerz}

Klinik für Chirurgie, St. Gallen, Switzerland
Background. Morgagni-Larrey hernia is a rare clinical entity among adults. It's clinical presentation is variable and unspecific. Symptoms can be misinterpreted and can lead to a delayed diagnosis. Computed tomography is the gold standard in fixing diagnosis. Definitive surgical management strategies have not yet been well established. Different surgical approaches and surgical techniques are described.

Methods. In our video we demonstrate a novel laparoscopic treatment concept applied in a patient with a giant Morgagni-Larrey hernia. An Endo-Close suturing device is used to close the hernial gap followed by a modificated intraperitoneal onlay mesh (IPOM) application.

Results. One $10 \mathrm{~mm}$ and three $5 \mathrm{~mm}$ trocars are used. Reducion of the hernia is performed laparoscopicaly. The hernial gap is closed using an Endo-Close suturing device to position resorbable threads through the hernial margins followed by a special extracorporal stiching technique. The reconstruction is secured by overlapping with a lightweight and partially absorbable mesh in onlay technique. Mesh fixation is performed using resorbable and non resorbable tuckers.

Conclusions. Our new technique is a feasible method in the treatment of a Morgagni hernia. The Endo-Close suturing device enables the closure of even bigger hernias in an entire laparoscopic way by causing a minimum of tissue trauma.

\section{Arbeitsgemeinschaft für Qualitätssicherung in der Chirurgie: Varia}

\section{8}

\section{Ein generisches webbasiertes Datenspeicherungssystem für Qualität- und Komplikationsmanagement}

\section{Girardi, J. Dirnberger, M. Giretzlehner}

RISC Software GmbH - Medical Informatics, Hagenberg, Austria

Jeder Qualitätssicherungs- und Komplikationsmanagementprozess ist stark abhängig von der Qualität der dafür erfassten Daten. Diese müssen sicher und strukturiert abgelegt und auf ihre Plausibilität sowie Gültigkeit geprüft werden. Dafür ist in der Regel ein professionelles Datenspeicherungssystem notwendig, welches mit entsprechenden Kosten und Entwicklungszeiten verbunden ist. Während die Struktur der zu erfassenden Daten stark von der medizinischen Domäne abhängt, bleiben die Grundanforderungen an ein solches System meist konstant: Eingabemasken zur Datenerfassung, Übersichtstabellen, Statistik- und Suchfunktion, Benutzerverwal- 
tung, Import- und Exportschnittstellen, hohe Verfügbarkeit, usw.

Um diesen immer wiederkehrenden Anforderungen nachzukommen, wurde ein hoch-generisches, selbstadaptives, webbasiertes Datenerfassungssystem entwickelt, welches es dem Benutzer erlaubt, die Struktur der zu erfassenden Daten einfach per Weboberfläche festzulegen. Aufgrund der darunterliegenden Meta-Datenstruktur kann das System beinahe beliebige Datenstrukturen erfassen. Die Anwendung selbst, in Form von Eingabemasken, Suchmasken und Übersichtstabellen, konfiguriert sich aufgrund dieser Benutzerdefinitionen. Da dieses System nur konfiguriert, und nicht entwickelt werden muss, ist es innerhalb weniger Stunden einsatzbereit. Ebenso ist das System offen für Änderungen während des laufenden Betriebes.

Unser System erlaubt es auch kleineren Institutionen oder Stationen ihr Qualitäts- und Komplikationsmanagement auf sauber strukturierten und sicher abgelegten Daten aufzubauen. Dies erhöht nicht nur die Qualität der Studien sondern senkt auch die Einstiegshürden für ein solches Vorhaben.

\section{9}

\section{Chirurgische Nachsorge beim Kolorektalen Karzinom - Österreichweite Umfrage der ACO-ASSO}

\section{Zitt}

Univ.-Klinik für Visceral-, Transplantations- und Thoraxchirurgie, Medizinische Universität Innsbruck, Innsbruck, Austria

Grundlagen. Bei PatientenInnen mit Kolorektalem Karzinom ermöglicht die Nachsorge die frühzeitige Erkennung von asymptomatischen Tumorrezidiven, was die Chance einer neuerlichen kurativen Therapie erhöht. Darüber hinaus wird die Nachsorge zur Beurteilung der Lebensqualität nach onkologischen Eingriffen, aber auch als Instrument zur chirurgischen Qualitätssicherung in der Onkologie herangezogen. Trotz dieser Fakten herrscht nach wie vor eine kontroverse Diskussion über die Intensität der Nachsorge, über die Untersuchungsintervalle und über die verwendeten Untersuchungsmethoden. Derzeit werden in verschiedenen Ländern von verschiedenen onkologischen Gesellschaften unterschiedliche Nachsorgeschemata beim Kolorektalen Karzinom propagiert.

Methodik. Basierend auf diesem Hintergrund haben wir in der Arbeitsgruppe „Colon/Rektum/Anus“ der ACOASSO beschlossen, das sogenannte „Nachsorge-Projekt beim Kolorektalen Karzinom“ zu initiieren. Im Rahmen dieses Projektes wird der Ist-Zustand der Nachsorge beim Kolorektalen Karzinom an allen chirurgischen Abteilungen Österreichs erhoben. Dazu wurde ein Fragebogen entwickelt, welcher an die chirurgischen Abteilungen Österreichs versandt wurde.
Ergebnisse. Die Ergebnisse der Umfrage werden präsentiert. Dabei wird im Besonderen auf die Rücklaufquote der Fragebögen, auf die Art und die Intensität der verwendeten Nachsorgeprogramme sowie Untersuchungsmethoden Wert gelegt.

Schlussfolgerungen. Die Hauptergebnisse dieser Umfrage stellen die Grundlage für ein standardisiertes chirurgisch-onkologisches Nachsorgeschema beim Kolorektalen Karzinom dar, welches dann von der ACO-ASSO als „Qualitätsstandard“ vorgeschlagen und österreichweit angewendet werden könnte.

\section{0}

Schmerz in der Chirurgie - eine interdisziplinäre Herausforderung

\section{Matzi ${ }^{1}$, K. Putz-Scheicher ${ }^{1}$, D. Ofner ${ }^{1}$, K. H. Tscheliessnigg ${ }^{1}$, A. Sandner-Kiesling ${ }^{2}$, F. M. Smolle-Jüttner ${ }^{1}$}

${ }^{1}$ Klinische Abteilung für Thorax-und Hyperbare Chirurgie, Universitätsklinik für Chirurgie, Medizinische Universität Graz, Graz, Austria; ${ }^{2}$ Universitätsklinik für Anästhesie und Intensivmedizin, Medizinische Universität Graz, Graz, Austria

Grundlagen. Schmerz ist ein allgegenwärtiges Thema der Chirurgie. Sowohl postoperativer Schmerz als auch Schmerz bei Tumorpatienten, der die Lebensqualität massiv beeinflusst, ist ein Problem des chirurgischen Alltages, das nur im interdisziplinären Management beherrschbar ist.

Methodik. Von Mai - Dezember 2010 wurde an der Universitätsklinik für Chirurgie unter Leitung des Projektmanagements Stabstelle QM-RM das Schmerzmanagement evaluiert. Von ärztlicher und pflegerischer Seite gemeinsam wurden abteilungsspezifische medikamentöse und nicht-medikamentöse Schmerztherapieschemata erarbeitet und optimiert. Zusätzlich wurden Physiotherapie und Psychotherapie ins Schmerzmanagement eingebunden. Von September - Oktober 2010 wurden 107 Patienten der Universitätsklinik für Chirurgie, Ärzte und Pflegepersonal bezüglich Information zur Schmerztherapie, Erfassung des Schmerzes, Umsetzung der Schmerztherapie und Messung der Ergebnisqualität befragt. Die statistische Auswertung erfolgte mittels $t$-test, ANOVA oder $\chi^{2}$-Test.

Ergebnisse. An allen Abteilungen wurde eine multiprofessionelle Arbeitsgruppe Schmerz implementiert, erfolgte eine quantifizierbare Schmerzerfassung und eine nachvollziehbare, adäquate und dokumentierte Schmerztherapie. Es zeigten sich $\mathrm{zu}$ wenig beachtete schmerzauslösende Ereignisse wie Entfernung von Drainagen oder Husten, nächtliche Therapielücken oder fehlende Schmerzäußerungen von älteren Patienten und Tumorpatienten. Alle evaluierten Abteilungen erfüllten die Kriterien, sodass die gesamte chirurgische Klinik im Dezember 2010 die Zertifizierung „Qualifizierte Schmerztherapie" durch Certkom erreichte. 
Schlussfolgerungen. Durch eine qualifizierte, interdisziplinäre Schmerztherapie rückt das Ziel „schmerzfreies Krankenhaus“ näher und verbessert sich die Patientenzufriedenheit.

\section{1}

\section{Ungeplante Revisionen in einer konsekutiven Serie von 251 Pankreasresektionen}

\section{U. Fröschl' ${ }^{1}$, O. Gangl ${ }^{1}$, T. Sautner ${ }^{2}$, R. Függer ${ }^{1}$}

${ }^{1}$ Krankenhaus der Elisabethinen/Chirurgische Abteilung, Linz, Austria; ${ }^{2}$ Krankenhaus der Elisabethinen/Chirurgische Abteilung, Wien, Austria

Grundlagen. Monitoring von Revisons-, Reinterventions- und Mortalitätsraten nach Pankreasresektionen.

Methodik. In einem Zeitraum von 10 Jahren (01-10) erfolgten an unserer Abteilung $n=251$ Pankreasresektionen. Patientendaten und ca. 60 Parameter zu Indikationsstellung, peri- und postoperativem Verlauf wurden prospektiv in einer Datenbank erfasst und retrospektiv ausgewertet. Sämtliche Indikationen zur Resektion wurden in die Analyse mit einbezogen (43\% Adeno Karzinom des Pankreas, $17 \%$ benigne Pankreastumoren, $17 \%$ sonstige maligne Tumoren, $17 \%$ chronische Pankreatitiden und 5,6\% neuroendokrine Tumoren.

Ergebnisse. Es wurde in $61,8 \%(n=155)$ eine pyloruserhaltende Duodenopankreatektomie, 22,3\% $(n=56)$ eine Linksresektion, 7,6\% ( $n=19)$ eine totale Pankreatektomie, in $6,4 \%(n=16)$ ein klassischer Kausch Whipple, in $1,2 \%(n=3)$ eine zentrale Pankreatektomie und in $0,8 \%$ $(n=2)$ eine Enukleation durchgeführt. Bei 6,4\% (16/251) der Patienten erfolgte eine operative Revision, bei $9,2 \%$ (23/251) eine Reintervention (11 CT gezielte Drainagen, 6 sonographisch gezielte Drainagen, 6 Angiographien). Die 30 Tage Mortalität liegt bei 2,4\% (6/251). Beim Adeno Karzinom konnte in $74 \%(n=108)$ ein R0 Status erreicht werden (23\% R1 $n=25,2,8 \%$ R2 Resektionen $n=3$ ).

Schlussfolgerungen. Die prospektive Dokumentation und Auswertung von Revisons-, Reinterventions- und Mortalitäsraten sind grundlegende objektivierbare Qualitätskriterien nach Pankreasresektion.

\section{2}

Effizientes Prozessmanagement verlangt Qualitätskontrolle: Erste Ergebnisse der Qualitätssicherungsstudie Hernienchirurgie $\left(\right.$ Herniamed $\left.^{\circledR}\right)$

F. Mayer ${ }^{1}$, F. Köckerling ${ }^{2}$, M. Lechner ${ }^{1}$, R. Bittner ${ }^{3}$, R. Fortelny ${ }^{4}$, D. Öfner-Velano ${ }^{1}$
${ }^{1}$ Universitätsklinik für Chirurgie, Paracelsus Medizinische Universität Salzburg, Salzburg, Austria; ${ }^{2}$ Klinik für Chirurgie, Visceral- und Gefäßchirurgie, Vivantes Klinikum Spandau, Berlin, Germany; ${ }^{3}$ Hernienzentrum EuromedClinic, Fürth, Germany; ${ }^{4} 2$. Chirurgische Abteilung, Wilhelminenspital, Wien, Austria

Grundlagen. Prozessmanagement setzt permanente Qualitätskontrolle und Benchmarking der Einzelergebnisse voraus. Im Gesundheitswesen wird dies zunehmend gefordert. In der Hernienchirurgie haben die Danish Hernia Database und das Nationale Schwedische Hernienregister hier erste strukturierte Ansätze dargestellt. Viele Fragen bleiben weiterhin unbeantwortet, valide Daten insbesondere im internationalen Vergleich fehlen.

Methodik. Die Qualitätssicherungsstudie Herniamed ist ein online-Register in das alle hernienchirurgischen Eingriffe auf freiwilliger Basis kostenlos eingegeben werden können, das follow-up der Patienten ist standardisiert. Risikofaktoren, Komorbiditäten, Voroperationen, Operationstechnik und unmittelbarer post-OP outcome werden im Detail dargestellt.

Ergebnisse. 2010 wurden von 95 aktiven Zentren 8.719 Patienten erfasst. Leistenhernien (70,2\%), Nabelhernie $(13 \%)$ und Narbenhernie $(10,8 \%)$ dominieren die Eingriffsdiagnosen. $22 \%$ der Eingriffe wurden ambulant, $77 \%$ stationär durchgeführt. 2,9\% aller Operationen waren Notfalleingriffe (Inkarzeration). Die mittlere Krankenhausverweildauer beträgt 3 Tage. Bei der Leistenhernie dominieren MIC-Verfahren (58,1\%), in 91,6\% aller Eingriffe wurde ein Mesh implantiert. In 31,9\% aller Leistenhernienreparationen konnte auf eine Fixation des Implantats verzichtet werden. Die post-OP Komplikationsrate beträgt $9,9 \%$, die Letalitätsrate $0,1 \%$. Die Rate an Re-Operationen beträgt $1,2 \%$.

Schlussfolgerungen. Die Qualitätssicherungsstudie Herniamed zeigt bereits nach einem Jahr valide Daten zur Versorgungsqualität. Mit zunehmendem Qualitätsdruck wird vermutlich die Bereitschaft steigen, dass sich weitere Kliniken dieser Qualitätsoffensive nicht zuletzt unter dem Aspekt zunehmender Spezialisierung und Zentrumsbildung anschliessen.

\section{3}

Qualitätsindikatoren am Beispiel der Schilddrüsenchirurgie - Ergebnisqualität bestimmt Effizienz, Produktivität und Ökonomie

\section{Hermann}

Kaiserin Elisabeth Spital der Stadt Wien, Wien, Austria

Grundlagen. „Outcome-Daten“ orientieren sich an diagnosespezifischen und therapierelevanten Qualitätsindikatoren, die effektive Parameter zur Bewertung des 
Therapieerfolges darstellen. In der Schilddrüsenchirurgie sind die Qualitätsindikatoren streng definierbar: Stimmbandnervlähmung, Nebenschilddrüsenunterfunktion, Nachblutung und Wundinfektionsrate; darüber hinaus Rezidivrate und Sterblichkeit.

Methodik. 30.142 Schilddrüsenoperationen der Jahre 1979 bis 2008 wurden nach diesen Qualitätsindikatoren ausgewertet, um folgende Parameter für eine zukünftige Kostenoptimierung zu bestimmen: minimal erforderliche Liegedauer, Einfluss von Komplikationen auf die Kosten, Langzeiterfolg der Therapie.

Ergebnisse. Folgekosten einer transienten Recurrensparese betragen bis 3300 Euro. Eine junge Patientin mit einem permanenten Hypoparathyreoidismus verbraucht in fiktiven 40 weiteren Lebensjahren 36.000 Euro an Therapiekosten. Die Kostenpauschale für eine operative Revision wegen Nachblutung beträgt ca. 360 Euro. Eine eigene medizinische Einzelleistung für eine Struma-Nachblutung existiert bis dato gar nicht. Eine operative Revision wegen einer Wundinfektion kostet ca. 380 Euro, bringt dem Krankenhaus ungefähr 1.800 Euro an Fallpauschale plus Zuschlag (LKF). Der inbegriffene einwöchige Krankenhausaufenthalt im KAV kostet 4.389 Euro.

Schlussfolgerungen. Gute Ergebnisqualität, hohe Heilungsrate, Senkung der Komplikationen und kurze Liegedauer bestimmen Effizienz und Produktivität und bergen ein hohes Einsparungspotential in sich. Kostensenkung basiert allerdings nicht nur auf komplikationsarmer Chirurgie sondern auf straffer Abteilungsorganisation mit gezielter OP-Planung, strukturierter prästationärer Abklärung und einem Aufnahme- und Entlassungsmanagement.

\section{4}

\section{Entwicklung einer hepatobiliären Datenbank in Kooperation mit der medizinischen Informatik - ein Modell zur Steigerung der Effizienz und Qualitätssicherung}

\author{
S. Stättner ${ }^{1}$, T. Höfler ${ }^{2}$, J. Karner ${ }^{1}$ \\ ${ }^{1}$ SMZ Süd, Kaiser Franz Josef Spital, Abteilung Chirurgie, \\ Wien, Austria; ${ }^{2}$ Technische Universität, Wien, Austria
}

Grundlagen. Die hepatobiliäre Chirurgie umfasst ein weites Spektrum an Krankheitbildern und erfordert in hohem Ausmass eine enge Kooperation mit unterschiedlichen Fachdisziplinen. Gerade im onkologischen Bereich fließen zahlreiche Variable sowohl in der Diagnostik als auch der Therapie in die anzuwendenden Algorithmen ein. Zum Beispiel muss zwischen Lebercirrhotikern und Patienten mit gesundem Leberparenchym unterschieden werden, nicht zuletzt da hier unterschiedliche Klassifikationen zur Anwendung kommen. Das erfordert einen äußerst komplexen Datenbank Aufbau.
Methodik. Vorstellung einer komplexen Datenbank für die tägliche klinische Anwendung.

Ergebnisse. Eine Access Datenbank wurde im Rahmen einer Diplomarbeit im Studium der medizinischen Informatik über einen Zeitraum von mehr als 6 Monaten erstellt. Der klinische Input und die Fragestellungen wurden vom Chirurgen vorgegeben und vom Informatiker umgesetzt. Anwendungen im täglichen Gebrauch der Nachsorge maligner wie benigner Erkrankungen, operative Daten, deskriptive Angaben als auch Qualitätsindizes (Erythrozytenverbrauch, Aufenthaltsdauer, Morbidität und Mortalität, etc.) wurden eingeflechtet. Einfache Statistiken sind jederzeit abrufbar und stehen abteilungsintern und für persönlichen Bedarf jederzeit zur Verfügung.

Schlussfolgerungen. Diese Datenbank soll ein Modell zur Qualitätssicherung darstellen und zeigt wie zukünftig Kooperationen und Synergien zwischen medizinischer Informatik und den täglichen klinischen Anwendern genutzt werden können.

\section{5}

\section{Berufsbild „Chirurg/in“}

\section{A. End, V. Helmreich}

Klinische Abteilung f. Thoraxchirurgie, Univ. Klinik f. Chirurgie, Medizinische Universität Wien, Wien, Austria

Grundlagen. Diese Studie versucht die Sichtweisen österreichischer Chirurgen über ihr eigenes Berufsbild zu beschreiben.

Methodik. Die Freitexte des Online-Fragebogens über „Arbeitsmodelle in der Chirurgie“ (Umfrage der Öst. Ges. f. Chirurgie 2009) wurden analysiert. Berichte über berufliche Zufriedenheit und Unzufriedenheit sowie arbeitsbezogene Verbesserungsvorschläge werden dargelegt.

Ergebnisse. $47 \%$ der Teilnehmer machten einen Freitexteintrag. Als Gründe für berufliche Zufriedenheit werden eine interessante Tätigkeit, die Arbeit mit dem Patienten, ein weites Operationsspektrum und Flexibilität bei der Arbeitseinteilung genannt. Fachärzte weisen eine höhere Zufriedenheitsrate auf. Als Ursachen für Unzufriedenheit werden zunehmende Bürokratie, Zeitmangel für wissenschaftliches Arbeiten, geringe Operationsmöglichkeiten und schlechte Organisationsstrukturen genannt.

Schlussfolgerungen. Über die Hälfte der österreichischen Chirurgen sind mit der Ausbildung und dem Beruf relativ zufrieden. Die Freitextmöglichkeit wurde von vielen Chirurgen zu kritischen Kommentaren genutzt, die ein Anstoß zu Reformen sein sollten. Viele Kollegen sehen die chirurgische Arbeit - auch bei schlechten Arbeitsbedingungen - als eine abwechslungsreiche, erfüllende Tätigkeit an. 


\section{Österreichische Gesellschaft \\ für Thorax- und Herzchirurgie: Thoraxchirurgie - Varia}

\section{6}

Long term follow up after minimal invasive repair for the correction of pectus excavatum: a single center experience

H. T. Maier ${ }^{1}$, F. Augustin ${ }^{1}$, A. Schwabegger ${ }^{2}$, A. Tontsch ${ }^{1}$, T. Schmid ${ }^{1}$, P. Lucciarini ${ }^{1}$

${ }^{1}$ Center of Operative Medicine, Department of Visceral, Transplant and Thoracic Surgery, Innsbruck, Austria; ${ }^{2}$ Center of Operative Medicine, Department of Plastic and Reconstructive Surgery, Innsbruck, Austria

Background. The aim of this study was to evaluate our results after the Nuss operation (minimal invasive repair of pectus excavatum). Attention was given to patient comfort with regard to surgical results and ability to cope with the implanted metal-bars in different daily situations.

Methods. From 2002 to 2009, 32 Nuss procedures were performed. The postoperative outcome was investigated by a questionnaire to assess individual overall-satisfaction (score 1-4). Physical ability (score 1-4) was recorded pre- and postoperatively. Different daily life situations were answered (score 1-6). Nine patients were lost for follow-up. Postoperative complications were depicted separately.

Results. Eighteen (78\%) patients were satisfied with the results achieving scores 1-2 (overall-satisfaction). Physical abilities without limitations were seen in 7 (31\%) patients prior to surgery, $3(13 \%)$ at discharge, 9 $(39 \%)$ at one year postoperatively and $21(92 \%)$ at time of inquiry. Categories daily life, profession, sports, social network, psychological acceptance and absence of complaints showed scores 1-2 in 23 (100\%), 22 (96\%) 21 (91\%), 21 (91\%), 21 (91\%) and 20 (87\%) patients. Scores 5-6 were seen in the categories psychological acceptance and absence of complaints (one patient each). No major postoperative complications requiring re-intervention were observed.

Conclusions. According to our questionnaire, surgical repair of pectus excavatum improves limitations on physical activity and overall satisfaction.

\section{7}

Early recurrence after minimally invasive and conventional lobectomy for early stage lung cancer - a single center comparative study

F. Augustin ${ }^{1}$, J. Bodner ${ }^{1}$, M. Fiegl ${ }^{2}$, H. Maier ${ }^{1}$, P. Lucciarini ${ }^{1}$, T. Schmid ${ }^{1}$
${ }^{1}$ Univ.-Klinik für Chirurgie, Innsbruck, Austria; ${ }^{2}$ Univ.-Klinik für Innere Medizin, Innsbruck, Austria

Background. Open lung lobectomy is the gold standard in early stage non-small cell lung cancer (NSCLC). However, video assisted thoracoscopic (VATS) lobectomy is getting more popular because of shorter hospital stay, less pain and earlier recovery. Oncologic equality still has to be proven. We report our data on tumor recurrence for VATS lobectomies in early stage NSCLC and compare these data to a historic group with open lung lobectomy.

Methods. Since 02/2009, 111 patients were scheduled for VATS lobectomy. Exclusion criteria for this study were benign disease, nodal invasion, tumor stage $>$ T2a, conversion to thoracotomy and neoadjuvant treatment. Comparison group consisted of 284 patients operated for early NSCLC (pT1-2N0M0) between 01/2000 and 12/2006 (TYROL Study).

Results. Fifty-four patients successfully underwent VATS lobectomy for early stage NSCLC. Median follow up was 12 (IQR 6.6-19.2) months. Tumor recurrence occurred in 2 patients (3.7\%; 2 distant metastases). 32/284 patients $(11.3 \%)$ had tumor recurrence after open lung lobectomy within their first postoperative year.

Conclusions. Even though follow-up is short, patients after VATS lobectomy show at least a comparable recurrence rate for early lung cancer compared to the open surgical approach. No nodal local recurrence occurred in the VATS series, indicating a proper lymph node dissection.

\section{8}

\section{Up-Staging vom klinischen zum pathologischen Tumor-Stadium nach VATS Lobektomie}

\section{J. Bodner, F. Augustin, T. Schmid \\ Univ.-Klinik für Chirurgie, Innsbruck, Austria}

Grundlagen. Die Therapie des nicht-kleinzelligen Bronchuskarzinoms wird primär auf Grund des klinischen Tumor-Stadiums entschieden wobei das Frühstadium (UICC I/II) eine Domäne der chirurgischen Therapie darstellt. Ein Up-Staging des pathologischen Stadiums im Vergleich zum klinischen kann - bei präoperativ korrekt durchgeführtem Staging - als Qualitätsnachweis der chirurgischen Therapie interpretiert werden.

Methodik. Retrospektiver Vergleich zwischen klinischem und pathologischem TNM/UICC Stadium nach VATS Lobektomie wegen NSCLC. Bei Patienten, bei welchen es zu einem up-Staging gekommen war, wurde das klinische Tumor-Stadium nochmals anhand der OriginalUntersuchungen reevaluiert. 
Ergebnisse. Von $2 / 09$ bis $1 / 11$ wurden 94 VATS Lobektomien wegen eines NSCLC im Frühstadium durchgeführt. Das klinische Tumorstadium wurde mittels KM-CT $(94,100 \%)$, PET $(n=82,87 \%)$ und invasiven Staging-Methoden (Mediastinoskopie und/oder EBUS-gezielte-transbronchiale Biopsie, $n=14,15 \%$ ) erhoben. 18 mal kam es zu einem pathologischen UpStaging, wegen eines höheren T-Faktors in 4 bzw. wegen eines höheren N Faktors in 14 (15\%) Fällen. Ein Shift von N0 zu N1, von N1 zu N2 und von N0 zu N2 trat 7, 1 bzw. 6 mal auf.

Schlussfolgerungen. Die zur Verfügung stehenden Methoden zum klinischen Staging des NSCLC sind unzureichend. Der Nachweis positiver N1 oder N2 Lymphknoten kann als Bestätigung der hohen Qualität der onkologischen Lymphknotendissektion bei der VATS Lobektomie interpretiert werden.

\section{9}

\section{Jahre minimal invasive anatomische Lungenresektionen - Innsbrucker Erfahrung}

\section{T. Schmid, J. Bodner, F. Augustin}

Univ.-Klinik für Chirurgie, Innsbruck, Austria

Grundlagen. Video-assistierte thorakoskopische (VATS) Lobektomien werden zum Standard in der Behandlung der Frühstadien des Lungenkarzinoms. Vor 2 Jahren wurde diese Technik auch in Innsbruck erfolgreich eingeführt. Wir präsentieren eine retrospektive Analyse der Patienten, die in diesem Zeitraum minimal invasiv in Innsbruck operiert wurden.

Methodik. Indikation, Zugang, Technik, peri- und postoperative Daten der Patienten wurden retrospektiv ausgewertet.

Ergebnisse. Insgesamt wurde bei 114 Patienten eine VATS Lobektomie geplant. Bei 3 Patienten lag eine benigne Erkrankung vor, 111 Patienten wurden wegen maligner Erkrankungen einer Lobektomie unterzogen. Bei 11 Patienten (9,6\%) musste auf ein offenes Vorgehen konvertiert werden. Gründe hierfür waren Blutungen in 5 Patienten und nicht-notfallmäßige Konversionen aus onkologischen/technischen Umständen in 6 Patienten. Der postoperative Nachbeobachtungszeitraum liegt median bei 12 Monaten. Rezidive traten bei 8,3\% der Patienten mit Lungentumoren auf, wobei die Rezidivwahrscheinlichkeit in der Gruppe der Patienten mit fortgeschrittenen Tumorstadien signifikant höher lag (pT12aN0M0 vs. pT2b-3N1-2M0, $p=0,05$ ).

Schlussfolgerungen. Die initialen peri- und postoperativen Ergebnisse sind vielversprechend. Derzeit zeigt sich das Verfahren onkologisch einer offenen Operation gleichwertig. Jene Patienten mit nicht-notfallmäßigen Konversionen werden eingehend präsentiert.

\section{0}

\section{Esophageal Leiomyomatosis 25 years after Myotomy for Achalasia}

\author{
A. Perathoner ${ }^{1}$, E. Laimer ${ }^{1}$, L. Veits ${ }^{2}$, G. Mikuz ${ }^{2}$, \\ W. Vogel $^{3}$, T. Schmid ${ }^{1}$, J. Pratschke ${ }^{1}$, R. Margreiter ${ }^{1}$, \\ H. F. Wykypiel ${ }^{1}$
}

${ }^{1}$ Medizinische Universität Innsbruck/Universitätsklinik für Visceral-, Transplantations- und Thoraxchirurgie, Innsbruck, Austria; ${ }^{2}$ Medizinische Universität Innsbruck/Institut für Pathologie, Innsbruck, Austria; ${ }^{3}$ Medizinische Universität Innsbruck/Universitätsklinik für Innere Medizin II (Gastroenterologie und Hepatologie), Innsbruck, Austria

Background. Esophageal leiomyomatosis is a rare hamartomatous disorder with proliferation of the smooth muscle layer of the esophagus. The resulting thickening of the esophageal wall causes stenosis of the gastroesophageal junction with slowly progressive dysphagia. Children and young adults are predominantly affected by this condition. Diagnosis is suspected by typical radiological findings in chest radiography, barium swallow and computed tomography.

Methods. We report about an unusual case of esophageal leiomyomatosis in a 30-year-old female patient with a history of achalasia with Heller myotomy and partial posterior fundoplication as a child.

Results. The patient presented with hematemesis and melena in the emergency unit. The radiological and endoscopic findings revealed a big circumferential tumor of the gastroesophageal junction creating a subtotal stenosis of the distal esophagus with prestenotic dilation with ulcerative esophagitis. The surgical treatment consisted of esophageal resection with gastric tube pull-up.

Conclusions. The diagnosis of leiomyomatosis was complicated by the unusual medical history of the patient and the large extension of the tumor mimicking a malignant neoplasm. To the best of our knowledge, this case report is the first description of the unusual coincidence of an achalasia as a child and an esophageal leiomyomatosis as an adult.

\section{1}

\section{Thymektomie bei Patienten mit Myasthenia Gravis}

\section{S. Buchner, S. Reich- Weinberger, D. Öfner- Velano, J. Hutter}

Universitätsklinik für Chirurgie Salzburg, Paracelsus Medizinische Universität, Salzburg, Austria

Grundlagen. Bei an Myasthenia gravis (MG) erkrankten Patienten wird die Indikation zur Thymektomie nachwievor kontrovers diskutiert. In dieser Arbeit wurde der 
subjektive therapeutische Effekt dieser Operation hinterfragt.

Methodik. An 28 Patienten mit MG wurde nach ausführlicher Diagnostik an einer neurologischen Abteilung (EMG, Tensilon Test, Antikörper gegen Acetylcholinrezeptorprotein, Thorax- Röntgen) und konservativer Therapie (Mestinon, Immurek, Prednisolon) eine Thymektomie durchgeführt (1999-2010). Postoperativ wurde anhand des MG QOL 15 der subjektive therapeutische Effekt erfragt.

Ergebnisse. 16 Frauen und 12 Männer mit einem durchschnittlichen Alter von 48,2 Jahren (23 bis 69 ), wurden überwiegend $(89,3 \%)$ videoassistiert thorakoskopisch (VATS) thymektomiert. Bei lediglich drei Patienten $(10,7 \%)$ wurde die Thymektomie transsternal durchgeführt. Histologisch traten bei fünf Patienten maligne Thymome, bei 11 unauffällige Thymusreste und bei neun Thymushyperplasien auf. Ein Viertel der Patienten berichteten über eine unveränderte Klinik, ein Drittel über eine Verschlechterung und die Mehrheit (43,75\%) über eine deutliche Verbesserung der MG assoziierten Symptome. 82, 5\% der operierten Patienten würden sich retrospektiv erneut einer Thymektomie unterziehen.

Schlussfolgerungen. Unsere Ergebnisse bestätigen bei dieser seltenen Indikation die der Literatur. Die Thymektomie, vorzugsweise minimal invasiv, stellt mangels alternativer Therapieoptionen einen wichtiger Bestandteil in einem Therapiekonzept für Patienten mit MG dar.

\section{2}

\section{SAGB: an in vivo model for the development of adenocarcinoma of the distal esophagus?}

\section{P. Gehwolf, T. Schmid, J. Pratschke, H. F. Wykypiel}

Medizinische Universität Innsbruck/Universitätsklinik für Visceral-, Transplantations- und Thoraxchirurgie, Innsbruck, Austria

Background. GERD is the principal underlying condition for the development of adenocarcinoma of the distal esophagus. Development of a hiatal hernia, gastroesophageal reflux, deterioration of the lower esophageal sphincter, dilation of the distal esophagus and consequently a defective motility of the lower esophagus with impaired clearance contribute to stasis, erosion, intestinal metaplasia, dysplasia and adenocarcinoma as a function of time. In some SAGB (Swedish Adjustable Gastric Banding) patients, all of the conditions mentioned above act together in order to form "the perfect storm".

Methods. A 50 year old male with a BMI of 43 received a SAGB for weight reduction in 1995. On follow-up, he suffered from erosive esophagitis and was treated with PPI and emptying of the band repeatedly. In 2010, an adenocarcinoma of the distal esophageal was diagnosed.
A thoracoscopic esophageal resection with gastric tube pull-up was performed.

Results. The postoperative course was uneventful despite several comorbidities.

Conclusions. For many pathophysiologic reasons, SAGB should be performed only in patients with excellent esophageal peristalsis, excellent compliance and meticulous follow-up. Detailed functional work-up of the upper GI and psychologic evaluation preoperatively could be prerequisites to prevent the development of adenocarcinoma of the lower esophagus after SAGB.

\section{Österreichische Gesellschaft für Kinder- und Jugendchirurgie: Neue Techniken in der Kinder- und Jugendchirurgie}

\section{3}

\section{Changes in treatment strategies} for congenital incomplete prepyloric diaphragm over the last four decades

\section{P. Gehwolf, P. Hechenleitner, C. Profanter, J. Hager,} B. Häussler

Univ.-Klinik Innsbruck, Visceral-, Transplantationsund Thoraxchirurgie, Abteilung für Kinder- und Jugendchirurgie, Innsbruck, Austria

Background. A congenital prepyloric diaphragm is a rare cause of congenital gastric outlet obstruction (GOO). It was first described in 1933. The pathogenesis is unknown but it is expected to be the result of a fetal vascular aberration. Over the years many different treatment options have been described.

Methods. We present five cases with different kinds of congenital prepyloric diaphragm in a time period of 40 years (1970-2010) and hence discuss changes in treatment strategies over the years. Treatment consisted of excision of the webs without pyloroplasty and recently we established a minimal invasive endoscopic treatment procedure.

Results. In all our patients the web could be removed without pyloroplastic or antrum resection even though the membranes were located next to the pylorus. Nevertheless the surgical approach may have several complications such as paralytic ileus, intestinal adhesions and surgical site infections. With the development of more minimal invasive treatment forms and the diminution of endoscopes the endoscopic incision or dilatation of antral webs in children became conceivably.

Conclusions. To guarantee the most sufficient and most minimal invasive treatment we acquired a new endoscope and changed our treatment strategies for con- 
genital incomplete prepyloric diaphragms into a minimal invasive endoscopic approach.

\section{4}

\section{Video-assisted thoracoscopic surgery}

\section{Pones, W. Krois, W. Rebhandl}

AKH Wien, Univ. Klinik f. Kinderchirurgie, Vienna, Austria

Background. We continuously expanded the indications for thoracoscopic surgery at our department. Therefore we reviewed our experience to assess the applications for video-assisted thoracoscopic surgery (VATS) at our institution.

Methods. We performed a retrospective review of all patients undergoing thoracoscopic surgery at our department from January 1, 1997 to September 16, 2010.

Results. During the period of the study 33 patients underwent 36 thoracoscopic operations. Mean age was 12.6 years (min. 4.07, max. 28.24). Sixty-four percent of the patients were male. VATS was used for bullae resection and decortication in 10 patients, pleural decortication for empyema was performed in 3 patients, sympathectomy for hyperhidrosis palmaris in 3 patients, atypical segmentresection for metastasis in 2 patients, thoracoscopy for hematothorax/seroma in 2 patients, bronchogenic cyst in 1 patient, suspicion for leucemic infiltrat in 1 patient, lymphangiomatous malformation in 1 patient, congenital diaphragmatic hernia $(\mathrm{CDH})$ in 1 patient and diagnostic procedures like biopsy in 9 patients. The mean length of postoperative hospitalization was $4.06 \pm 3.23$ days. Mean time of postoperative chest tube drainage was $3.05 \pm 3.15$ days.

Conclusions. Video-assisted thoracoscopic surgery is a safe technique with a low conversion rate. Complications are minimal but exact knowledge and training are mandatory.

\section{5}

Single shot transversus abdominis plane block for postoperative analgesia is superior than conventional administration of NSAIDs in children after laparoscopic surgery

\section{A. Albokrinov ${ }^{1}$, A. Pereyaslov ${ }^{2}$, A. Dvorakevich ${ }^{1}$ \\ ${ }^{1}$ Children's Regional Clinic Hospital, Lviv, Ukraine; \\ ${ }^{2}$ Medical University, Lviv, Ukraine}

Background. It is well known that adequate postoperative (PO) analgesia is the cornerstone for the uncom- plicated PO period. Administration of non-steroidal antiinflammatory drugs (NSAIDs) after minor surgery often gives satisfactory level of PO comfort, but their use may lead to some adverse effects. Applying of epidural block in these patients may lead to complications related to anesthesia itself. The transversus abdominis plane (TAP) block is a newly described peripheral block involving the nerves of the anterior abdominal wall. The results of clinical trials with TAP block are encouraging.

Methods and results. Overall 23 pediatric patients were randomized into TAP group $(n=11)$ and NSAIDs group $(n=12)$. In TAP group $0.6 \mathrm{ml} / \mathrm{kg}$ of $0.25 \%$ bupivacaine was injected into transversus abdominis plane in a blind manner (two pops) via triangle of Petit immediately after surgery. Patients of NSAIDs group received ketoprofen $0.5 \mathrm{mg} / \mathrm{kg}$ at induction of anesthesia and $3 \mathrm{mg} / \mathrm{kg} /$ day after surgery. Mean Visual Analog Scale values were significantly better in TAP group then in NSAIDs group $(1.3 \pm 0.4$ vs. $3.4 \pm 0.6, p<0.05)$. Besides this children in NSAIDs group received 4 rescue doses of morphine $(0.1 \mathrm{mg} / \mathrm{kg}$ s.c. $) v s$. 0 doses in TAP group.

Conclusions. TAP block is more effective method of PO analgesia then administration of NSAIDs.

\section{6}

\section{Laparoskopische Cholezystektomien im Kindes- und Jugendalter}

\section{G. Pomberger, E. Maier, A. Rokitansky}

Kinder- und Jugendchirurgie SMZ-Ost, Wien, Austria

Wir berichten über die laparoskopische Technik der Cholezystektomie im Kindes- und Jugendalter anhand von 96 Fällen im Alter von 4-18 Jahren (Mittel 14,01-57 Mädchen und 39 Burschen) und einem Körpergewicht von 16-118 kg (Mittel 63,4 kg). Eingegangen wird einerseits auf die kongenitalen hämatologischen Erkrankungen, die schon im Vorschulalter zur Gallensteinbildung und damit verbundenen Komplikationen führen können, andererseits auf das Gallensteinleiden bei älteren Kindern und Jugendlichen als Ausdruck einer Zivilisationskrankheit wie auch bei Erwachsenen (fettreiche Ernährung, Bewegungsmangel).

Hervorgehoben wird der Einzug der laparoskopischen Operationstechnik auch bei Patienten im frühen Kindesalter mit naturgemäß sehr kleinem Peritonealraum. Aus der Routine der laparoskopischen Technik am Kind ergeben sich auch große Vorteile beim jugendlichen Patienten, der aus chirugischer Sicht wie ein Erwachsener zu operieren ist.

Darüberhinaus führen wir großzügig eine begleidende Appendektomie durch, unter der Voraussetzung, dass sich diese problemlos über die Zugänge für die Cholezystektomie möglich ist, und keinen zusätzlichen Port erfordert. 


\section{7}

\section{Immunphenotypical characterization of appendiceal and peripheral blood lymphocytes in children}

\section{T. Benkoe ${ }^{1}$, S. Baumann ${ }^{1}$, P. Angleitner ${ }^{1}$, M. Meier ${ }^{1}$, E. Horcher ${ }^{1}$, G. Zlabinger ${ }^{2}$, W. RebhandI ${ }^{1}$ \\ ${ }^{1}$ Klinische Abteilung für Kinderchirurgie, Medizinische Universität Wien, Wien, Austria; ${ }^{2}$ Institut für Immunologie, Medizinischen Universität Wien, Wien, Austria}

Background. This study was conducted to determine the immunologic cellular composition in human appendicitis comparing peripheral blood and appendiceal tissue.

Methods. Blood samples and appendiceal tissue were from children undergoing appendectomy. Immunphenotypical characterization of lymphocytes of peripheral blood and appendiceal tissue were performed using FACS analysis. According to the extent of appendiceal inflammation we divided our patients in four different groups (phlegmonous, gangraenous, perforated and non-inflamed). Lymphocyte subsets (T-helper cells: CD4; cytotoxic T-cells: CD8; natural killer cells: CD56 and Blymphocytes: CD19) were correlated with the degree of appendicitis.

Results. We included 49 patients (median age: 11.4 years; range: $3.9-17$ ). All tested lymphocyte subsets could be detected in appendiceal tissue. According to the inflammatory state of the appendix we could demonstrate a recruitment of lymphocytes from the peripheral blood into the inflamed appendix. Subset analysis of lymphocytes showed increasing levels of $\mathrm{CD}^{+}$and $\mathrm{CD}^{+} \mathrm{T}$-cells in the appendiceal tissue and a reduction of both cell types in the peripheral blood.

Conclusions. This study provides new data showing a recruitment of peripheral blood lymphocytes in the inflamed appendix. The progress in inflammation seems to be perpetuated by increased migration of $\mathrm{CD}^{+}{ }^{+} \mathrm{T}$-helper cells and $\mathrm{CD}^{+}$cytotoxic T-cells to the appendix.

\section{8}

An easy and novel procedure to overcome appendicostomy insufficiencies

\section{A. Reck, R. Fartacek, S. Baumann, E. Horcher}

Medical University of Vienna, Vienna, Austria

Background. Appendicostomies for an antegarde continence enema (ACE) represent a practical way to help children with stool incontinence become clean. It is technically simple to perform and allows a direct connection from the skin to the cecum through an appendix stoma. Stomal stenosis are the most common complications and consequent noncompliance of colonic irrigation leading to recurrent incontinence.

Methods. We present 2 cases in which stomal stenosis was overcome with the help of a Chait Trapdoor (R) device in an easy to perform procedure.

Results and conclusions. We present a simple but useful alternative to re-operation in patients presenting with stricture of a previously placed appendicostoma. Life quality significantly increased in these two patients. The Chait Trapdoor button represents and easy to perform and effective device for this purpose.

\section{9}

\section{Klassifizierung und Richtlinien zum Management von Neugeborenen mit "Congenital Pouch Colon"}

\author{
A. Saxena ${ }^{1}$, P. Mathur ${ }^{2}$ \\ ${ }^{1}$ Medizinische Universität Graz, Graz, Austria; \\ ${ }^{2}$ SMS Medical College, Jaipur, India
}

Grundlagen. Das Ziel dieser Studie war die Erstellung eines Leitfadens der Managementstrategie in der Behandlung von Congenital Pouch Colon (CPC).

Methodik. Vom 1995 bis 2007 wurden 426 Patienten wegen anorektaler Fehlbildungen am RNT Medical College, Udaipur, behandelt. CPC wurde bei 80 Patienten festgestellt. Die 80 Patienten wurden in 5 Typen und Managementstrategien - abhängig von der Lokation des sackförmigen Reservoirs und seiner Beschaffenheit zum Zeitpunkt des Operierens - kategorisiert.

Ergebnisse. Bei Typ-1 und Typ-2 CPC bestimmte die Beschaffenheit des Pouch (ischämisch oder gesund), ob eine einstufige (Pouchexzision und anorektaler Durchzug), oder dreistufige Behandlungsmethode (Ileostomie, Pouch-Coloplastik mit anorectalem Durchzug und Ileostomie-Rückverlagerung) als förderlich angesehen wurde. Bei Typ-3 und Typ-4 CPC wird eine dreistufige Behandlungsmethode (Pouchexzision mit Colostomie, anorectaler Durchzug und Colostomie-Rückverlagerung) empfohlen. Bei Typ-5 wird einer dreistufigen Behandlungsmethode (distale Pouchexzision mit proximaler Pouch-Coloplastie zusätzlich zur Ileostomie, anorectaler Durchzug und Colostomie-Rückverlagerung) der Vorzug gegeben.

Schlussfolgerungen. CPC-Management unter Einbeziehung der vorgeschlagenen Klassifizierung zeigt klare Richtlinien für die Patientenbehandlung in Bezug auf die anatomische Morphologie des Pouch auf. Bei Typ-1 und Typ-2 CPC bestimmt die Beschaffenheit des sackförmigen Reservoirs (gesund oder ischämisch) die Operationsstrategie. Bei Typ-3, Typ-4 und Typ-5 sind klare Richtlinien für das Pouch-Management nachvollziehbar dargestellt. 


\section{$249 a$}

\section{Frakturbehandlung bei Kindern und Jugendlichen mittels resorbierbarer Implantate}

\section{J. Schnöll, J. Bauer, G. Schimpl}

UK für Kinder und Jugendchirurgie, Salzburg, Austria

Grundlagen. Seit den 60-er Jahren werden resorbierbare Materialien in den Traumatologie verwendet um Frakturen zu stabilisieren. Zunehmende Qualitätsverbesserung und Bedienerfreundlichkeit der Implantate veranlassten uns im Jahr 2005 ebenfalls mit diese Art der Osteosynthese $\mathrm{zu}$ beginnen.

Methodik. Wir stellten die Indikation bei Patienten jenseits des 13 Lebensjahres die eine Epiphysenfraktur/lösung an der unteren Extremität zeigten. An der oberen Extremität kam die Methode beim Abriss des Epicondylus ulnaris bei Ellbogenluxationen zum Einsatz. In den Jahren 2005-2010 wurden so 11 Patienten versorgt. 9 Patienten an der unteren Extremität, 2 an der oberen Extremität. Bei allen Patienten mit Verletzungen an der unteren Extremität wurde nach dem konventionellen Röntgen eine CT/MRI Untersuchung der betroffenen Region durchgeführt. Die Versorgung wurde minimal invasiv durchgeführt. (Geschlossen Reposition, Einbringen der Schrauben mittels kanülierter Instrumente). An der oberen Extremität erfolgte die Reposition offen nach konventioneller Röntgendiagnostik.

Ergebnisse. Alle Patienten zeigten eine gute knöcherne Konsolidierung, seitengleichen Bewegungsumfang und gehen ihren Interessen und Aktivitäten im täglichen Leben ohne Einschränkungen nach.

Schlussfolgerungen. Die Methode gewährleistet ausreichende Stabilisierung der Fraktur. Wir beobachteten keine Wachstumsstörung. Als größten Vorteil sehen wir aber das Ausbleiben einer 2. Narkose zur Metallentfernung. Als Nachteil gelten die (noch) hohen Herstellungskosten und die Unsichtbarkeit des Implantates während der Operation.

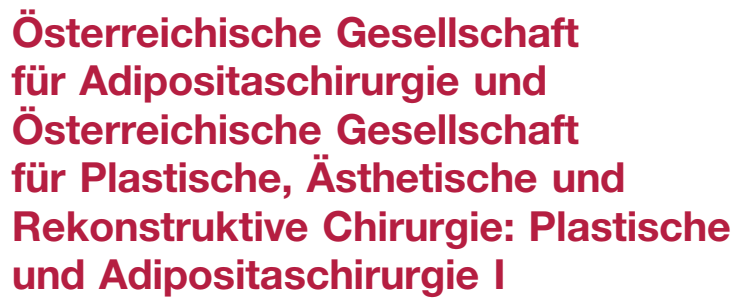

\section{0}

\section{Bodylifting des unteren Stammbereiches. Eine Analyse von 70 Fällen}

\section{Koller, P. Mayr, T. Hintringer}

Abteilung für Plastische-,Ästhetische-, und Rekonstruktive Chirurgie Kh Barmherzige Schwestern Linz, Linz, Austria

Grundlagen. Das Bodylifting des unteren Stammbereiches kann zu einer erheblichen ästethischen und funktionellen Verbesserung führen. Mit einer resultierenden Narbe wird der Abdomenbereich inkl. Schamhügelbereich, der seitliche und vordere Oberschenkelbereich, und der Glutealbereich gestrafft.

Methodik. An unserer Abteilung führten wir seit 2009 an siebzig Patienten ein Bodylifting des unteren Stammbereiches durch. Das superfizielle Fasziensystem diente dabei als Präparations- und Suspensionsschicht.

Ergebnisse. Im Vergleich mit der Literatur war die Komplikationsrate gering. Lediglich zwei Patienten hatten eine Nachblutung, die revidiert werden musste. Die Seromrate lag unter $8 \%$. Es kam zu keinen Hautnekrosen oder tiefen Wunddehiszenzen.

Schlussfolgerungen. Das untere oder zentrale Bodylifting ist eine ausgedehnte Operation mit grosser Wundfläche. Gerade deshalb ist die Schonung der tiefen Lymphgefässe sinnvoll. Das superfizielle Fasziensystem ist ein geeignetes Gewebe zur Präparation, Straffung und Formung dieser Körperregion.

\section{1}

\section{Unteres Bodylift - subjektive Ergebnisbewertung durch die Patienten und psychologische Statusdiagnostik des Körperbilds}

\author{
H. B. Kitzinger, A. Pittermann, R. Wenger, B. Karle, \\ M. Frey \\ Abt. für Plastische und Rekonstruktive Chirurgie, \\ Medizinische Universität Wien, Wien, Austria
}

Grundlagen. Ausgedehnte Hautschürzen nach massiver Gewichtsreduktion führen beim PatientInnen zu den 
typischen Problemen einer subjektiven Unattraktivität. Durch eine ausgedehnte Straffungsoperation, wie dem unteren Bodylift, erwartet man sich trotz langer Narben eine deutliche Verbesserung des körperlichen Wohlbefindens. Diese Hypothese wird am eigenen PatientInnengut retrospektiv überprüft.

Methodik. Zur Erhebung der postoperativen Patientenzufriedenheit, der Körperwahrnehmung und dem Körperempfinden wurden die international validierten Fragebögen FBK20, FBeK und ein eigens entworfener Fragebogen verwendet. Aufgrund der kleinen Patientenpopulation beschränkte sich die statistische Auswertung auf eine deskriptive Analyse.

Ergebnisse. Von 30 PatientInnen, die zwischen 2007 und 2009 operiert worden sind, konnten 28 PatientInnen in die Studie eingeschlossen werden. $90 \%$ der PatientInnen würden die Operation noch einmal durchführen lassen. Alle acht, häufig mit Hautüberschüssen assoziierten Parameter, zeigten sich postoperativ signifikant gebessert. Weder die postoperative ablehnende Körperbewertung noch die vitale Körperdynamik zeigten ein auffälliges Ergebnis im Vergleich zu den Kontrollgruppen. Konträr dazu fand sich in der Skala Attraktivität/Selbstvertrauen ein niedriger Prozentrang, welcher einer geringen Zufriedenheit mit dem eigenen Körper entspricht.

Schlussfolgerungen. PatientInnen beschreiben eine große Zufriedenheit mit dem unteren Bodylift. Aufgrund der unterschiedlichen Ergebnisse in den Fragebögen scheint aber die Wahl des Fragebogens zur postoperativen Bewertung eines Bodylifts eine entscheidende Rolle zu spielen.

\section{2}

\section{Body contouring nach postbariatrischer Chirurgie - ein Erfahrungsbericht}

\section{F. Sokullu, D. E. Afshar, R. Bartsch, W. Michlits, M. Deutinger}

Krankenanstalt Rudolfstiftung - Abteilung für Plastische und Wiederherstellungschirurgie, Vienna, Austria

Grundlagen. Vielen Adipositas-Patienten gelingt es nicht durch Ernährungsumstellung und Bewegung eine adäquate Gewichtsreduktion zu erzielen. In diesen Fällen führt alleine die operative Intervention zum Ziel. Mit Zunahme der Adipositaschirurgie rückt auch immer mehr die Plastische Chirurgie in den Mittelpunkt.

Methodik. An unserer Abteilung wurden zwischen 2008 und 201071 post-bariatrische Patienten behandelt. In 43 Fällen wurde eine Dermolipektomie, in 15 ein Bodylift, in 5 eine Oberschenkelstraffung, in 4 eine Oberarmstraffung und in einem Fall eine Beltlipektomie durchgeführt. Das Alter der Patienten reichte von 24 bis 69 .
Ergebnisse. Der postoperative Verlauf war in den meisten Fällen komplikationslos. In 7 Fällen $(6 \times$ Dermolipektomie, $1 \times$ Bodylift) musste eine Revision durchgeführt werden. In 4 Fällen kam es zur Ausbildung eines Seroms $(2 \times$ Dermolipektomie, $2 \times$ Bodylift) und in 5 Fällen zu einer Wundheilungsstörung $(2 \times$ Dermolipektomie, $3 \times$ Bodylift). Die Patienten nach Oberschenkel- und Oberarmstraffungen, sowie Beltlipektomie zeigten keine postoperativen Komplikationen. Komplikationen, wie Infektionen, Thrombosebildung sowie Lungenembolie wurden nicht beobachtet.

Schlussfolgerungen. Die Adipositaschirurgie hat sich in den letzten Jahren sehr rasch weiter entwickelt und ermöglicht Patienten mit morbider Adipositas völlig neue Möglichkeiten. Einen wesentlichen Stellenwert nimmt dabei die Plastische Chirurgie ein, denn ohne „Body contouring" wären viele Patienten zwar schlanker, würden sich aber in ihrem Körper nicht wohl fühlen.

\section{3}

\section{Bruststraffung nach massiver Gewichtsabnahme}

\section{Koller, P. Mayr, T. Hintringer}

Abteilung für Plastische-, Ästhetische-, und Rekonstruktive Chirurgie Kh Barmherzige Schwestern Linz, Linz, Austria

Grundlagen. Die Gewebeerschlaffung nach massiver Gewichtsabnahme ist individuell unterschiedlich. Dies zeigt sich am deutlichsten im Brustbereich. Je nach Ptosegrad, Ausmaß der Hauterschlaffung und Beschaffenheit des Eigengewebes wird die für den Patienten am besten geeignete Operationsmethode gewählt.

Methodik und Ergebnisse. Von 70 Patienten, die an unserer Abteilung ein zentrales oder unteres Bodylifting erhielten, wurde bei 26 (23 Frauen, 3 Männer) eine Bruststraffung durchgeführt. Verschiedene Methoden kamen dabei zur Anwendung: Die Hautstraffung erfolgte über periareoläre, vertikale oder inverse T-Narben. Die Gewebestraffung erfolgte mit Eigengewebe oder Fremdgewebe bzw. einer Kombination aus beiden. Die Mamillenstielung wurde cranial, craniomedial, oder inferior gewählt.

Bei der Straffung mit Eigengewebe wurden auch Techniken mit lokalen Lappen zur Autoaugmentation angewandt.

Das eigene Patientengut wird analysiert und die verschiedenen Operationsmethoden erläutert.

Schlussfolgerungen. Auf Grund der Heterogenität der Brusterschlaffung, die sich im Patientengut nach starker Gewichtsabnahme zeigt, sollte aus mehreren Methoden die für den Patienten am besten geeignete ausgewählt werden. In einer Vielzahl der Fälle kommen dabei herkömmliche Methoden der Bruststraffung zur Anwendung. In ausgewählten Fällen eignen sich Techniken der Autoaugmentation mit lokalen Lappenverschiebungen. 


\section{4}

\section{Erfahrungen bei der Behandlung des Oberkörpers bei MWL-Patienten}

\section{G. Helml, G. M. Huemer}

Allgemeines Krankenhaus Linz, Linz, Austria

Grundlagen. Der Oberkörper und Oberarme stellen für den Patienten nach massiver Gewichtsabnahme (MWL) nach der Abdominalregion und den medialen Oberschenkeln die wichtigste Körperregion für einen konturverbessernden Eingriff dar. Während in der unteren Körperhälfte das lower body lifting eine Vielzahl der Problemzonen verbessert, bedarf es bei der oberen Körperhälfte meist innovativerer Lösungen um ein optimales Ergebnis zu erzielen.

Methodik. Alle MWL-Patienten, die eine konturverbessernde Operation im Bereich des Oberkörpers erhalten haben, wurden in einer prospektiv geführten Datenbank identifiziert.

Ergebnisse. Bei 39 Patienten (4 männlich) wurde zwischen April 2006 und Jänner 2010 eine konturverbessernde Operation am Oberkörper durchgeführt. Es handelte sich bei jedem Eingriff um zumindest eine Korrektur von 2 verschiedenen anatomischen Regionen. Postoperative Ergebnisse inklusive Komplikationen werden präsentiert.

Schlussfolgerungen. Zur optimalen Behandlung des Oberkörpers bei MWL-Patienten müssen innovative und kombinierte Verfahren zum Einsatz gebracht werden. Insbesondere die weibliche Brust stellt hier hohe Anforderungen und nur durch Kenntnis mehrerer Techniken kann eine ästhetische Brustform rekonstruiert werden. Das upper body lifting ist eine sichere Operation, welches eine profunde Verbesserung der Kontur des Oberkörpers in nur einer Sitzung erlaubt.

\section{5}

\section{Konturverbessernde Operationen nach} massivem Gewichtsverlust: Was sind die Erkenntnisse aus dem letzten Jahrzehnt aus plastisch-chirurgischer Sicht?

\section{H. B. Kitzinger, B. Karle, M. Frey}

Abt. für Plastische und Rekonstruktive Chirurgie, Medizinische Universität Wien, Wien, Austria

Grundlagen. Retrospektive Analyse der operativen Strategie und der Ergebnisse anhand eines selektionierten Patientengutes aus zehn Jahren.

Methodik. Zwischen 1995 und 2005 wurden an 283 Patienten konturverbessernde Eingriffe nach Gewichtsabnahme durch Diät bzw. bariatrischer Operation durchge- führt. 127 Patienten erfüllten die Einschlusskriterien der Studie. Es erfolgte die Auswertung der Patientenakten sowie der telefonischen Patientenbefragung in Bezug auf Gewichtsverhalten und Patientenzufriedenheit.

Ergebnisse. 127 Patienten (105 Frauen, 22 Männer) in einem durchschnittlichen Alter von 39,4 Jahren und nach einem durchschnittlichen Gewichtsverlust von $45,3 \mathrm{~kg}$ (BMI 28,6) unterzogen sich insgesamt 164 plastisch-chirurgischen Operationen. An 123 Patienten wurden eine Abdominoplastik bzw. abdominelle Fettschürzenresektion durchgeführt, gefolgt von Mastopexie (8), subkutaner Mastektomie (3), Oberschenkelstraffung (8), Oberarmstraffung (4), Gesäß-Lifting (1) und der Dermolipektomie am Rücken (1) bzw. Mons pubis (1). $19 \%$ aller Patienten sahen ihre Erwartungshaltung an das Ergebnis nicht erfüllt.

Schlussfolgerungen. Die Korrekturbedürftigkeit aus Sicht der Patienten betrifft mit großem Abstand den abdominellen Gewebeüberschuß, gefolgt von der Mammaptose und dem Gewebeüberschuß am medialen Oberschenkel. Aufgrund des immer größer werdenden Patientenguts mit St. p. massiver Gewichtsreduktion nach bariatrischem Voreingriff, führen regional begrenzte Straffungsoperation immer weniger zum Erfolg. Aus diesem Grund werden an unserer Abteilung die regional begrenzten Straffungsoperation seit 2007 zunehmend durch untere und obere Bodylift-Operationen ergänzt.

\section{6}

\section{Die vertikale Oberschenkelstraffung beim MWL-Patient}

\section{G. Helml, G. M. Huemer}

Allgemeines Krankenhaus Linz, Linz, Austria

Grundlagen. Der Oberschenkel gehört zu den Regionen, die am stärksten von einer massiven Gewichtsabnahme (MWL) betroffen sind. Die klassische Oberschenkelstraffung mit horizontaler Narbe in der Leiste ist bei diesem Patientengut nicht geeignet, um eine zufriedenstellende Konturierung zu erreichen.

Methodik. Alle MWL-Patienten, die eine vertikale Oberschenkelstraffung erhalten haben, wurden in einer prospektiv geführten Datenbank identifiziert.

Ergebnisse. Bei 38 Patientinnen wurde zwischen Juli 2007 und Jänner 2010 eine vertikale Oberschenkelstraffung durchgeführt. Es handelte sich dabei meist um den 3. Schritt in einem 3-Stufen Plan für Patienten nach massiver Gewichtsabnahme. Präsentiert werden ein Op-Video, postoperative Ergebnisse und Komplikationen.

Schlussfolgerungen. Der Oberschenkel stellt meist den letzten Schritt in der Behandlung des postbariatrischen Patienten dar. Nichtsdestotrotz handelt es sich aufgrund der oft ausgeprägten Hauterschlaffung um eine ästhetisch wichtige Region. Die vertikale Oberschenkel- 
straffung führt zu einer zirkulären Verbesserung über die gesamte Länge des Oberschenkels und ist deshalb, trotz einer nicht unbeträchtlichen Komplikationsrate, die Methode der Wahl bei dieser Patientengruppe.

\section{7}

\section{Hautverschluss mit Prineo (Dermabond Protape) bei Abdominoplastik. Eine offene prospektive, randomisierte, kontrollierte klinische Studie}

\section{Parvizi, I. Justich, M. V. Schintler, S. Spendel}

Klinische Abteilung für Plastische, Ästhetische und Rekonstruktive Chirurgie Universitätsklinik für Chirurgie Medizinische Universität Graz, Graz, Austria

Grundlagen. Die Dermatolipektomie zählt zu den aufwändigsten und den Körper am meisten belastenden ästhetisch-chirurgischen Eingriffen. Da es sich bei diesem Eingriff um große Wundverhältnisse handelt, kommt es bei der Annäherung der Gewebeschichten zu großen Zugspannungen an der Haut (Gefahr der Wunddehiszenz). Das Hauptaugenmerk in dieser Studie wurde auf die Wundverhältnisse sowie auf das kosmetische Ergebnis der Narbe gerrichtet.

Methodik. In zwei randomisierten Gruppen von jeweils 20 PatientInnen wurde ein unterschiedliches Hautverschluss-System angewendet. In beiden Gruppen wurde die Spannung der Haut mittels tiefer kutaner und/oder intrakutaner Nähte vor dem definitiven Verschluss reduziert. Die Gruppe 1 wurde mittels Prineo (Dermabond Protape), die zweite Gruppe mittels Steristrips versorgt. Die Beurteilung der Wundverhältnisse (14-tage post-operativ) erfolgte anhand des „Hollander Wound Evaluation and Cosmesis Scale“. Sechs Monate post-operativ erfolgte eine symptomatische sowie kosmetische Narbenbeurteilung (subjektiv sowie objektiv) durch den „Patient Scar Assessment Scale“ und „Vancouver Scar Scale“. Die Scores der beiden Behandlungsgruppen (Prineo und Steristrips) wurden mit einem zweiseitigen $T$-Test (Signifikanzniveau $5 \%$ ) auf Unterschied untersucht.

Ergebnisse. Der „Hollander Wound Evaluation and Cosmesis Scale“ lag nach Hautverschluss mittels Prineo bei durchschnittlich 8,45 Punkte im Vergleich zu Steristrips mit 6,15 Punkte. Hier zeigte sich ein statistisch signfikanter Unterschied ( $p$-value $=0,004$ ) in den Wundverhältnissen sowie im kosmetischen Ergebnis 14d postoperativ zu Gunsten von Prineo (Dermabond Protape). Bei der Beurteilung der kosmetischen Beschaffenheit der Narbe 6-Monate post-operativ anhand des „Vancouver Scar Scale" wurde mittels Prineo (Dermabond Protape) ein Mittelwert von 3,60 Punkte, im Vergleich zur herkömmlichen Methode 4,45 Punkte erzielt. Statistisch gesehen zeigte sich auch hier zu Gunsten von Prineo (Dermabond Protape) ein signifikanter Unterschied $(p$-value $=0,03)$. Im „Patient Scar Assessment Scale“
6-Monate post-operativ wurde durch Prineo (Dermabond Protape) ein Mittelwert von 8,45 Punkte gegenüber der herkömmlichen Methode 11,45 Punkte erzielt. Der statistische Unterschied ( $p$-value $=0,03$ ) in beiden Gruppen war wiederum signifikant für Prineo (Dermabond Protape).

Schlussfolgerungen. Die Ergebnisse dieser Studie zeigen, dass der Hautverschluss durch Prineo (Dermabond Protape) eine vernünftige Alternative in Bezug auf verbesserte Wundverhältnisse sowie kosmetischen Erscheinungsbildes gegenüber zur herkömmlichen Methode ist.

\section{8}

\section{Plastisch-chirurgische Operationen nach Adipositaschirurgie: Seltener als Erwartet}

\section{S. Shakeri-Leidenmühler, M. Poglitsch, R. Kefurt, A. Bohdjalian, F. Langer, G. Prager, F. X. Felberbauer}

Department of Surgery, Medical University, Vienna, Austria

Grundlagen. Erfolgreiche bariatrische Eingriffe erfordern oft plastisch-chirurgische Folgeeingriffe. Unbedingte Voraussetzung ist ein stabiles Körpergewicht, daher erfolgen diese Operationen frühestens 1,5 bis zwei Jahre nach der Adipositasoperation. Folgeeingriffe kommen oft schon bei Erstvorstellungen zur Sprache und stellen an PatientInnen, Behandler und öffentliches Gesundheitssystem beträchtliche Anforderungen. Wir haben im Krankengut unserer Klinik die Häufigkeit, den Erfolg und die Zufriedenheit mit postbariatrischen Eingriffen erhoben.

Methodik. Strukturiertes Telefoninterview mit folgenden Fragen:

- Haben Sie nach der Magenoperation eine (oder mehrere) plastische Operationen durchführen lassen?

- Wenn JA:

- Welche Operation(en)?

- Sind Sie mit dem Ergebnis zufrieden?

- Wenn NEIN:

- Planen Sie eine solche Operation, wenn ja, welche?

Ergebnisse. PatientInnen: insgesamt: 930

Befragt: 621

Folgeeingriff: 92 geplant: 68 abgelehnt: 14

Operationen: insgesamt: 92

Abdominalplastik: 65

unteres Bodylift/Oberschenkelstraffung: 25

Oberarmstraffung: 7

Andere: 4

Zufriedenheit:

Hoch: $52 \%$

Mässig: $30 \%$

Niedrig: $18 \%$

Schlussfolgerungen. Obwohl sich die Mehrzahl der PatientInnen bereits bei der Erstvorstellung nach Folgeeingriffen erkundigt, wurden lediglich $14,8 \%$ der PatientInnen operiert. 52\% der PatientInnen waren mit 
dem Ergebnis sehr zufrieden. Viele (11\%) der befragten PatientInnen überlegen noch einen Eingriff; Bei 2,3\% wurde ein Folgeeingriff bereits einmal abgelehnt, vor allem wegen instabilem Körpergewicht oder noch nicht ausreichendem Gewichtsverlust.

\section{Proktologie}

\section{9}

\section{Chirurgie der Analfistel in einem proktologischen Zentrum}

\section{Haunold, K. Sorko, M. Wunderlich}

$\mathrm{KH}$ der Barmherzigen Schwestern, Wien, Austria

Grundlagen. Das Spektrum der analen Fistelchirurgie umfasst Ringdrainage, Fistulotomie und Fistulektomie mit Sphinkternaht und Schleimhautlappenplastik (FEP). Ziel dieser retrospektiven Studie war es, diese Eingriffe an unserer diesbezüglich spezialisierten Abteilung zu analysieren.

Methodik. Von 2003 bis 2010 wurden im KH der BHS Wien 851 Fisteloperationen durchgeführt. Der Anteil an Rezidiveingriffen war überdurchschnittlich hoch.

Soferne nicht offenbar ein submucöser Verlauf vorlag, wurde primär nie fistulotomiert, sondern stets der Gang in Form einer Fistulektomie vollständig exzidiert, was in vielen Fällen eine Präparation bis an Levator ani implizierte. Verblieb unter dem Exzisionskanal eine dünne Gewebsbrücke ohne substantielle Muskulatur, so wurde diese im Sinne einer Fistulotomie gespalten. In allen anderen Fällen wurde, wann immer möglich, eine FEP durchgeführt. Bei Abszessen oder unklarer anatomischer Situation wurde zunächst eine Vessel-loop Ringdrainage durchgezogen.

Ergebnisse. Intraoperative Komplikationen waren extrem selten. Nachblutungsrisiko in den ersten 24 Stunden unter $2 \%$.

Rezidive manifestierten sich nahezu immer schon in den ersten sechs Wochen danach. Eine postoperative faekale Inkontinenz war selten.

Schlussfolgerungen. Unser Prinzip der vollständigen Excision jedes Fistelganges, einschließlich seines in spitzem Winkel verlaufenden hohen cranialen Anteils resultiert einerseits in einem großen Anteil an FEP-Eingriffen, andererseits in einer niedrigen Rezidivrate. Aus unserer Sicht ist dieses gründliche chirurgische Vorgehen Therapien wie plug oder VAC vorzuziehen.

\section{0}

\section{Normal values for high resolution anal manometry}

\section{S. Gellersen, A. Dinnewitzer, D. Öfner-Velano}

Universitätsklinik für Chirurgie, Salzburg, Austria

Background. Approximately up to $25 \%$ of the population is affected by disorders of the anorectum and pelvic floor. Anal 3 Dimensional High-resolution manometry (3D-HRM) allows a more detailed analysis than conventional anal manometry. The purpose of this study was to define the essential features of 3D-HRM and establish normative values.

Methods. 30 volunteers without any symptoms of anorectal disorder and normal defecation pattern were investigated by normal HRM with a circumferential solid-state probe with $4.2 \mathrm{~mm}$ diameter including 10 sphincter sensors followed by 3D-HRM with 16 circumferential sensing regions including 256 total sensors, $10 \mathrm{~mm}$ diameter. Results for resting pressure, squeeze, bear down and sensation with an air and water filled balloon were recorded.

Results. A total of 29 patients were studied. Simultaneously recorded resting, squeeze, and relaxation pressures showed the two methods to be correlated although 3 dimensional HRM gives a much more detailed resolution. Patient comfort was higher with the flexible $4.2 \mathrm{~mm}$ catheter. Insertion of the $10 \mathrm{~mm}$ catheter including the central balloon could be a problem in patients after colorectal surgery.

Conclusions. These preliminary observations demonstrate that anorectal 3D-HRM is much more detailed compared to conventional HRM, especially for the detection of partial sphincter insufficiency.

\section{1}

\section{Langzeitergebnisse nach individuell maßgeschneiderter Therapie durch Hämorrhoidalarterienligatur (HAL) mit oder ohne zusätzliche Mukopexie}

\author{
M. Scheyer ${ }^{1}$, W. Herrnhof ${ }^{1}$, H. Pokorny ${ }^{2}$, G. Rollinger ${ }^{1}$, \\ S. Lancee ${ }^{1}$, E. Antonietti ${ }^{1}$, L. Brotzge ${ }^{1}$ \\ ${ }^{1}$ LKH Bludenz, Bludenz, Austria; ${ }^{2}$ LK Wiener Neustadt, Wiener \\ Neustadt, Austria
}

Grundlagen. Die Hämorrhoidalarterienligatur (HAL) in Kombination mit Mukopexie (=RAR) bietet für den Patienten Vorteile hinsichtlich geringerer postoperativer Schmerzen, kürzerem Spitalsaufenthalt und höherer Zufriedenheit im Vergleich zum derzeitigen Goldstandard - der konventionellen Hämorrhoidektomie. 
Ziel dieser retrospektiven Studie sind die Langzeitergebnisse nach HAL bei Patienten mit Hämorrhoiden im Stadium II wie auch nach RAR bei Patienten mit Hämorrhoiden im Stadium III oder IV.

Methodik. Nach retrospektiver Analyse von insgesamt 408 Patienten betrug das mediane Follow-up 6,5 Jahre. Die Nachuntersuchung erfolgte mittels anonymem Fragebogen, als Zielparameter wurden die erneute Protrusion von Hämorrhoidalknoten, Blutung, Juckreiz und Schmerz definiert.

Ergebnisse. 290 Patienten $(71,1 \%)$ waren beschwerdefrei, 118 Patienten $(28,9 \%)$ gaben persistierende Beschwerden an, 95 Patienten $(23,3 \%)$ erneut aufgetretene beziehungsweise weiterhin bestehende Hämorrhoidalknoten.

Anhaltende Blutungen, Juckreiz und Schmerzen beschrieben 113 Patienten (27,7\%). Ein Patient klagte postoperativ über geringgradige Inkontinenzbeschwerden.

Schlussfolgerungen. Sowohl die HAL bei 2.gradigen Hämorrhoiden als auch die Kombination von HAL und Mukopexie bei 3. und 4.gradigen Noduli erwiesen sich in der Langzeitbeobachtung als effektive und komplikationsarme Operationsmethoden.

\section{2}

\section{Fistulectomy with primary sphincter reconstruction: the new kid on the block for complex fistula in ano?}

\section{K. Wolff, C. Gingert, M. Adamina, L. Marti}

Klinik für Chirurgie, St.Gallen, Switzerland

Background. The ideal approach for high transsphincteric and suprasphincteric fistula in ano is controversial. The anal advancement flap (AF) servesas abenchmarkprocedurehoweveritssuccess rate is reportedly disappointing with a healing rate between 50 and $98 \%$ and as low as $29 \%$ for primary and recurrent fistula, respectively. The recently proposed anal fistula plug could not live up to the expectations with a success rate falling below $50 \%$. In this context, low recurrence rates have been reported after fistulectomy via sphincter division and immediate sphincter reconstruction (FSR) an approach that was formerly much opposed because of a feared poor sphincter function. This report aims at investigating FSR as performed inadedicated coloproctology unit.

Methods. FSR was performed as a single stage fistulectomy including sphincter division along the full length of the fistula tract, and immediate sphincter reconstruction. All patients were routinely followed up at 3 and 6 months in the outpatient clinic Fistula recurrence reoperation anal incontinence, perineal complaints and patient satisfaction (worst 0 best 10 ) were assessed by a structured telephone interview.
Results. From January 2009 to December 2010 we performed a FSR in 11 patients ( 2 female) of a total of 183 operated on in our department for a fistula in ano. All FSR patients presented with a high transphincteric (7) or suprasphincteric fistula (4), and 9 of them (82\%) underwent repeat fistula surgery. Perioperative and 30-day surgical morbidity was nil. Median length of stay was 6 days (range 3-8). Overall, 8 out of 11 (73\%) fistula remained closed at a median follow-up of 19 months (range 3-24). Fecal continence was good, with two patients reporting occasional flatus incontinence one of whom had also slight soiling (Wexner scores of 6 and 7). 3 patients complained from residual perianal pain (1-2 on a scale from 0 to 10) and further 4 reported non specific perineal symptoms (e.g. itching) at final follow-up. Mean and median patient satisfaction was 8 (range 5-10)

Conclusions. These preliminary results suggest that FSR is a safe procedure of high interest in the treatment of complex and recurrent fistula in ano. So far functional outcomes have been good and patients' satisfaction high Pending further investigations FSR may have the potential to become the procedure of choice for complex anal fistula.

\section{8}

Erreger- \& Resistenzspektrum von Infektionen einer chirurgischen Intensivstation im 10-Jahres-Vergleich 1996-2005 im Rahmen des mikrobiologischen Routinemonitorings

\section{S. Arndt ${ }^{1}$, P. Mroczkowski ${ }^{1}$, H. Lauf ${ }^{2}$, U. Lodes ${ }^{1}$, G. Weiß ${ }^{3}$, H. Schulz ${ }^{1}$, H. Lippert ${ }^{1}$, F. Meyer ${ }^{1}$}

${ }^{1}$ Universitätsklinikum A.ö.R., Klinik für Allgemein-, Viszeral\& Gefäßchirurgie, Magdeburg, Germany; ${ }^{2}$ Universitätsklinikum A.ö.R., Institut für Mikrobiologie, Magdeburg, Germany; ${ }^{3}$ Städtisches Klinikum, Klinik für Intensivtherapie, Magdeburg, Germany

Grundlagen. Prospektive Datenerfassung \& retrospektive Auswertung konsekutiver mikrobiologischer Untersuchungen (chirurgische ITS: 1996/2002/2004 \& 2005) zur Charakterisierung i) 10-Jahres-Verlauf (1996 vs. 2005); ii) mögliche Alterationen bei abgeschafftem Pavillonsystem \& neuer Zimmeraufteilung (2002 vs. 2004) nach Klinikneubau.

Methodik. Erfassung/Auswertung des Keimspektrums mit Spezifizierung (Anzahl/Anteil) einzelner Grup- 
pen (Gram-positive/-negative Erreger/Pilze) sowie separat bei den 3häufigsten Materialgruppen (Blut-/Urinkultur, Wundabstriche) incl. Resistenzbestimmungen selektierter Erreger.

Ergebnisse. Blutkulturen - kontinuierliche Verringerung Gram-positiver Erreger von $74,3 \%$ auf $45,2 \%$ \& relativer Anstieg der Pilze von 4,8\% auf 19,3\% (19962005: $p<0,001 / 2002-2004: \quad p=0,037)$. Abstriche: Signifikanter Abfall des Pilzprozentsatzes von 20,6\% auf 12,9\%; dafür Anteilvergrößerung Gram-positiver Erreger von $43,2 \%$ auf $57,5 \%$ (jeweils $p<0,001$ ). Spektakulär ist der Anstieg relevanter Gram-negativer Erreger in Blutkulturen (E.-coli-Anteil von 6,5\% auf 45,4\%; Klebsiellen von $11,5 \%$ auf $24,2 \%$ [1996-2005: $p<0,001 / 2002-2004$ : $p=0,244]$ - ähnlich signifikante Änderung [1996-2005: $p=0,014 / 2002-2004: p=0,007$ ] bei Abstrichen).

Schlussfolgerungen. Systematisches mikrobiologisches Langzeitmonitoring erscheint unentbehrlich, da i) zügige Therapieentscheidungen im intensivmedizinischen Behandlungsregime nach Erregerlage möglich sind, ii) mikrobielle Erregernachweise für Infektionsdiagnosen abrechnungstechnisch relevant werden, iii) erst längerfristig Alterationen des Keimspektrums erkennbar (MRSA/Pilze) \& iv) simultan Resistenzentwicklungen zu ermitteln sind (MRSA/ESBL-Stämme bei Enterobakterien, Fluconazol-resistente Pilze). Das kann institutionell eine infektionsbiologisch-hygienische \& kostenbestimmende/ gesundheitspolitische Relevanz erlangen sowie insbesondere fatale Verläufe abwenden helfen, nicht zuletzt durch begründbaren Zusatzaufwand (z.B. Isolationsmaßnahmen, kostenträchtige Ersatz-/Ausweichmedikation) mit zu erzielender Abdeckung/Vergütung.

\section{Hauptsitzung - Erweiterte Tumorchirurgie 2, Chirurgie im hohen Senium}

\section{0}

\section{Curative resections of pancoast tumors}

\author{
K. Hoetzenecker ${ }^{1}$, P. Altmann ${ }^{1}$, A. Z. Aliabadi ${ }^{2}$, \\ G. Lang ${ }^{1}$, C. Aigner ${ }^{1}$, S. Taghavi ${ }^{1}$, W. Klepetko ${ }^{1}$ \\ ${ }^{1}$ Department of Thoracic Surgery, Vienna, Austria; \\ ${ }^{2}$ Department of Cardiac Surgery, Vienna, Austria
}

Background. Pancoast tumors are a rare disease entity associated with a very poor prognosis. Due to their infiltrating nature into the thoracic wall, big brachiocervical vessels and nerval structures complete resection of the tumor is technically challenging. However, after induction therapy a resection with curative intent is feasible in most cases.
Methods and results. We retrospectively evaluated patients presenting with a Pancoast tumor at our department from 1998 to 2011. A total of 47 patients were included in the analysis, $88 \%$ of patients received an induction therapy. Most of the patients' tumors were classified as stage $\mathrm{T} 4$. In order to achieve an R0 resection, 6 laminectomies, 6 vascular grafts, 11 plexus resections and 4 clavicula resections had to be performed. The 30 -day-mortality-rate was $2 \%(n=1)$, morbidity rate was acceptable. Overall-survival was 1-year: 80\% 3-year: 54\% 5-year: 38\%, median DFI was 19 months after operation. $\mathrm{N}$ status, histological response after induction therapy were prognostically relevant, however, the extent of resection had no impact on prognosis.

Conclusions. Our experience shows that even advanced tumors of the pulmonary apex can be radically resected with good clinical results. A close co-operation between pneumologist, oncologist and orthopaedic/neuro-surgeon is pivotal in order to provide patients with a potentially curative treatment.

\section{1}

\section{Ergebnisse der Leberresektion beim metastasierten Magenkarzinom Gibt es ein Risikokollektiv?}

\section{W. Schildberg, R. Croner, V. Schellerer, W. Hohenberger, S. Merkel, A. Perrakis}

Chirurgische Klinik der Universität Erlangen/Nürnberg, Erlangen, Germany

Grundlagen. Generell gelten hepatisch metastasierte Magenkarzinom als prognostisch ungünstig. Ziel dieser Studie war es, einen potentiellen operativ-kurativen Ansatz zu untersuchen.

Methodik. Im Universitätsklinikum Erlangen-Nürnberg wurden im Zeitraum von 1972-2008 insgesamt 31 Patienten mit einem hepatisch metastasierten (synchron oder metachron) Magenkarzinom behandelt. Das mediane Alter betrug 66 Jahre (35-84 J) und das Verhältnis männlich zu weiblich lag bei 2:1. Alle Daten wurden prospektiv für das Krebsregister Erlangen erhoben und retrospektiv untersucht.

Ergebnisse. In $68 \%$ der Fälle konnten atypische oder anatomische Segmentresektionen durchgeführt werden. Größere Eingriffe im Sinne einer Hemihepatektomie (rechts/links) wurden h bei $32 \%$ der Patienten durchgeführt. Die R0-Resektionsrate betrug $74 \%$. Die postoperative Komplikationsrate lag bei $16 \%$, die Krankenhausletalität bei $6 \%$. Die 5-Jahresüberlebensrate aller operierten Patienten war $13 \%$. Patienten mit metachroner Metastasierung hatten ein signifikant besseres 5-Jahresüberleben als Patienten mit synchronen Lebermetastasen $(0 \%$ vs. $29 \%, p<0,001)$. Patienten mit solitären Lebermetastasen zeigten ein signifikant besseres 
medianes Überleben als Patienten mit multiplen Lebermetastasen ( $21 \mathrm{~m} v s .4 \mathrm{~m}, p<0,005)$.

Schlussfolgerungen. Das hepatisch metastasiertes Magenkarzinoms stellt ist im Hinblick auf unseren erhobenen Daten nicht unbedingt eine Palliativsituation dar, da in $13 \%$ der Fälle die 5-Jahresüberlebensmarke erreicht wurde. Besonders scheinen Patienten mit metachronen, solitären Lebermetastasen zu profitieren. Weitere kontrollierte Studien sollten durchgeführt werden.

\section{2}

\section{The influence of the hepatic venous pressure gradient on postoperative complications after liver resection for hepatocellular carcinoma}

\section{S. Stremitzer ${ }^{1}$, D. TamandI ${ }^{1}$, K. Kaczirek ${ }^{1}$, J. Maresch ${ }^{2}$,} B. A. Payer ${ }^{3}$, A. Ferlitsch ${ }^{3}$, T. Grünberger ${ }^{1}$

${ }^{1}$ Medical University Vienna/Department of General Surgery, Vienna, Austria; ${ }^{2}$ Medical University Vienna/Clinical Institute of Pathology, Vienna, Austria; ${ }^{3}$ Department of Gastroenterology and Hepatology, Medical University Vienna, Vienna, Austria

Background. Portal hypertension seems to increase the risk of postoperative complications after liver resection for hepatocellular carcinoma (HCC). The value of preoperative assessment of the hepatic venous pressure gradient (HVPG) to identify portal hypertension is unclear.

Methods. We retrospectively analyzed 132 patients who underwent liver resection for HCC between 2000 and 2009 at the Department of General Surgery, Medical University Vienna.

Results. In 39 (29.5\%) patients, the HVPG was preoperatively assessed. Mean HVPG was $6.4( \pm 3) \mathrm{mmHg}$ and $4.3( \pm 1.4) \mathrm{mmHg}$ in patients with and without postoperative complications, respectively $(p=0.028)$. The complication rate significantly differed at a cut-off of $5 \mathrm{mmHg}$ (52.4\% vs. $85.7 \% ; p=0.045)$. In patients with an HVPG $>5 \mathrm{mmHg}$, higher grades of liver fibrosis occurred more frequently ( $p=0.004)$. They also had a higher rate of postoperative liver dysfunction $(5.6 \% v s .38 .5 \%, p=0.022)$ and suffered more frequently from postoperative ascites (14.3 vs. $50 \%, p=0.022$ ). Furthermore, their median length of stay in hospital was significantly longer $(8(4-20)$ vs. 11 (7-26) days, $p=0.034$ ). Postoperative morbidity did not differ compared to patients without preoperative HVPG assessment $(p=0.14)$.

Conclusions. Preoperative HVPG assessment predicts liver fibrosis and postoperative complications. Postoperative morbidity was not influenced by preoperative HVPG measurement in this analysis.

\section{4}

Begleitappendektomie bei großen colorektalen Eingriffen- Standard oder unnötiges zusätzliches Trauma? Eine retrospektive Analyse 382 histologischer Präparate

\section{R. Exner, M. Sachsenmaier, A. Stift}

Univeristätsklinik für Chirurgie, Wien, Austria

Grundlagen. Bei Operationen zur kurativen Behandlung kolorektaler Karzinome wird häufig eine Begleitappendektomie durchgeführt. Die Entscheidung zur Durchführung dieses zusätzlichen Eingriffes wird an vielen chirurgischen Abteilungen subjektiv durch den zuständigen Operateur getroffen, ein Goldstandard liegt bis jetzt nicht vor.

Methodik. In dieser retrospektiven Studie wurden die Daten von 1352 konsekutiven Patienten, die wegen kolorektaler Karzinome an der Medizinischen Universität Wien in den Jahren 1993 bis 2009 operiert wurden, gesammelt und die histopathologischen Ergebnisse der Begleitappendektomiepräparate evaluiert. Außerdem wurden Komplikationen und Kosten im Zusammenhang mit dem Zusatzeingriff analysiert.

Ergebnisse. $316(23,37 \%)$ Patienten waren aufgrund einer Appendizitis in der Vergangenheit zum Zeitpunkt der Operation bereits appendektomiert. Eine Begleitappendektomie wurde bei $380(28,11 \%)$ Patienten durchgeführt, nur $86(22,63 \%)$ dieser hatten eine reguläre Histologie ihrer Appendix, bei 289 (76,05\%) Patienten zeigte sich ein pathologischer Befund und bei 9 (2,37\%) der Patienten fand sich eine Neoplasie.

Der zusätzliche Eingriff hatte in keinem Fall Komplikationen zur Folge. Der Kosten- und Zeitaufwand war vernachlässigbar.

Schlussfolgerungen. Die Begleitappendektomie ist ein sicherer Eingriff und sollte als Standard Rahmen großer kolorektaler Tumoroperationen integriert werden, da pathologische Veränderungen inklusive Neoplasien der Appendix häufig sind und so zukünftige Operationen vermieden werden können.

\section{5}

\section{Laparoskopische Chirurgie des kolorektalen Karzinoms in hohem Alter}

\section{J. Sauer}

Karolinen-Hospital Hüsten, Arnsberg, Germany

Grundlagen. Die Ergebnisse der laparoskopischen Chirurgie in hohem Alter sind in der Literatur unterschiedlich. Besonders bei komplexeren Techniken, wie der 
kolorektalen Karzinomchirurgie sind differente Ergebnisse veroeffentlicht.

Methodik. Alle wegen eines kolorektalen Karzinoms laparoskopisch resezierten Patienten zwischen 2008 und 2010 wurden prospektiv analysiert. Es wurden zwei Altersgruppen gebildet: unter 75 Jahre $(n=86)$ und ab 75 Jahre $(n=46)$.

Ergebnisse. Nach ASA zeigte sich ein Vorteil $(p<0,03)$ fuer die juengere Gruppe (ASA II vs. ASA III). Das UICCStadium war im Median UICC II. Die Operationszeit war in der Gruppe der aelteren Patienten kuerzer (155 vs. 179 Minuten; n.s.). Bei $13 \%$ der juengeren Patienten und bei $2 \%$ der aelteren Patienten wurde umgestiegen $(p<0,01)$. Nahtinsuffizienzen traten bei den juengeren Patienten in $4 \%$ auf, bei den aelteren Patienten in $3 \%$ (n.s.). $2 \%$ der ueber 74-jaehrigen und $1 \%$ der unter 75-jaehrigen Patienten verstarben (n.s.).

Schlussfolgerungen. Die laparoskopische kolorektale Karzinomresektion ist bei Patienten im hohen Alter ebenfalls sicher. Die in der Literatur beobachtete hoehere Rate an Anastomoseninsuffizienzen und die hoehere Mortalitaet konnte durch unsere Daten nicht bestaetigt werden. Die kuerzere Operationszeit und die geringere Umsteigerate sind der Lernkurve geschuldet, in deren Anfang die juengeren Patienten operiert wurden.

\section{6}

\section{Die Cholezystektomie im hohen Senium - ist 90 das neue 70 ?}

\author{
A. Dubecz, M. Langer, R. Stadlhuber, M. Schweigert, \\ H. J. Stein \\ Klinikum Nürnberg, Klinik für Allgemein-, Viszeral- und \\ Thoraxchirurgie, Nürnberg, Germany
}

Grundlagen. Über-Neunzigjährige sind die am schnellsten wachsende Bevölkerungsschicht im westlichen Europa. Trotz steigender Zahlen bei Gallenblasenleiden ist die elektive Cholezystektomie immer noch kontrovers diskutiert.

Methodik. Eine retrospektive Datenauswertung der Jahre 2004 bis 2009 aller Cholezystektomiepatienten über 90 Lebensjahre wurde durchgeführt. In diesem Zeitraum erfolgten insgesamt 3007 Choloezystektomien. Es wurden Co-Morbiditäten, OP-Indikation, Operationsverfahren, intraoperativer Befund, Histologie, sowie perioperative Morbidität und Mortalität ausgewertet.

Ergebnisse. 22 Über-Neunzigjährige wurden im untersuchten Zeitraum cholezystektomiert. Acht (33\%) Patienten hatten Diabetes, 12 (50\%) Bluthochdruck und 10 (42\%) eine koronare Herzerkrankung. 19 (86\%) Patienten wurden notfallmäßig operiert. Ein Patient wurde nach erfolgloser konservativer Therapieversuche operiert, nur 2 Patienten wurden elektiv operiert. 13 (59\%) Patienten wurden laparoskopisch operiert, bei dreien erfolgte die Konversion und 8 (36\%) Patienten wurden primär offen operiert. Histologisch zeigte sich in $14(64 \%)$ Fällen eine gangränöse Cholezystitis. Der durchschnittliche Krankenhausaufenthalt betrug 10 Tage (4-23 Tage). Ein Patient erlitt eine Cystikus-Stumpf-Insuffizienz und ein Patient verstarb im postoperativen Verlauf. Konversionsrate, Anteil offener OP-Verfahren, Anteil fortgeschrittener histologischer Befunde, ASAScore und Krankenhausaufenthalt waren signifikant höher im Vergleich zum Gesamtpatientengut.

Schlussfolgerungen. Unsere Ergebnisse zeigen, dass die Cholezystektomie auch im hohen Senium mit akzeptabler Morbidität und Mortalität sicher durchführbar ist. Das Pateintenalter alleine stellt keine Kontraindikation zur elektiven Cholezystektomie dar.

\section{Österreichische Gesellschaft für Kinder- und Jugendchirurgie: Komplikationsmanagement}

\section{9}

\section{Chirurgische Komplikationen der komplizierten Pneumonie im Kindesalter}

\section{Pones, S. Baumann, E. Horcher}

AKH Wien, Univ. Klinik f. Kinderchirurgie, Vienna, Austria

Grundlagen. Ziel dieser Studie ist die Erhebung aufgetretener Komplikationen in der chirurgischen Behandlung einer komplizierten Pneumonie bei Kindern, um in Zukunft die Entscheidungsfindung in der Therapie dieser komplexen Erkrankung zu erleichtern.

Methodik. Retrospektive Analyse aller Kinder, die zwischen August 1996 und März 2010, aufgrund einer komplizierten Pneumonie einen thoraxchirurgischen Eingriff erhalten haben.

Ergebnisse. Es wurden 56 Patienten (61 \% männlich) identifiziert. Das mittlere Alter betrug 5,89 Jahre. In 22 Patienten war die Pathologie links, in 28 rechts und in 2 bilateral. Die mittlere Zeit bis zur Entlassung betrug 14,07 Tage. $55 \%(n=31)$ erhielten 1 Intervention, $34 \%(n=19)$ erhielten 2, $9 \%(n=5)$ erhielten 3 und $2 \%(n=1)$ erhielten 4. Bei den Interventionen handelte es sich um 3 Pleurapunktionen, 56 Bülaudrainagen, 4 videoassistierte Thorakoskopien, 3 Minithorakotomien und 25 Thorakotomien. Bei letzteren wurde zusätzlich zur Dekortikation in 7 Fällen eine Bronchusnaht bzw. Fistelklebung, in 2 Fällen eine Lobektomie, in je einem Fall eine atypische Segmentresektion bzw. Lingularesektion, in einem Fall eine Parenchymnaht nach iatrogener Verletzung im Rahmen der Bülaudrainagenlegung und in einem Fall eine Rekonstruktion der Vena subclavia nach iatrogener Durchtrennung im Rahmen der Dekortikation durchgeführt. 
Schlussfolgerungen. Eine exaktere Planung der chirurgischen Therapie soll in Zukunft die hohe Anzahl an Folgeeingriffen reduzieren.

\section{0}

\section{Komplikationsmanagement einer Miliartuberkulose mit rezidivierenden Pneumothoraces und Kavernenbildung bei einem 14 Monate alten Jungen}

\section{K. Rothe ${ }^{1}$, S. Lau ${ }^{2}$, K. Magdorf ${ }^{3}$ \\ ${ }^{1}$ Klinik für Kinderchirurgie, Charite, Berlin, Germany; ${ }^{2}$ Klinik für Pädiatrie m.S. Pulmonologie und Immunologie, Charite, Berlin, Germany; ${ }^{3}$ Klinik für Pädiatrie, Helios Klinikum Emil von BehringCharite, Berlin, Germany}

Wir berichten über den ungewöhnlichen Fall eines 14 Monate alten Jungen mit einer Miliartuberkulose und rezidivierenden Pneumothoraces infolge einer Kavernenbildung im Bereich der der linken Lunge. Nach mehreren reanimationspflichtigen Zuständen infolge des wiederholten mediastinalen Shifts und unbefriedigenden Drainageanlagen erfolgte die chirurgische Sanierung mit Thorakotomie und Lingularesektion einschließlich der Kaverne. Der postoperative Verlauf war aus chirurgischer Sicht unproblematisch. Die initiale T-Zell Funktionsstörung erholte sich nach 6 Wochen. Das Kind entwickelte indessen ein IRIS (immune reconstitution inflammatory syndrome), was weitere Sauerstoff- und Prednisolontherapie für einige Wochen nach sich zog. Nach 1 Jahr präsentierte sich das Kind vollständig gesund.

\section{1}

\section{Pulmonary complications of acute hematogenous osteomyelitis}

\section{A. Pereyaslov, N. Opykan, O. Borova}

Medical University, Lviv, Ukraine

Acute hematogenous osteomyelitis (AHO) potentially serious disease, especially in cases of the pulmonary complications development. Timely diagnosis and adequate antibiotic therapy may prevent these complications.

Among 47 patients with AHO, the pulmonary complications had $13(27.7 \%)$ patients. Laboratory data assessed included white blood cell (WBC) count, C-reactive protein (CRP), and cultures from blood and pus from the lesion site. The highest CRP levels (158.82 $\pm 112.71 \mathrm{mg} / \mathrm{dL}$ ) were noted in patients with lung involvement vs. $96.44 \pm 66.32 \mathrm{mg} / \mathrm{dL}$ in other patients. Staphylococcus aureus caused AHO and pulmonary complications in 11 (84.6\%) patients and in 4 of them the multi-resistant St. aureus was identified. Pseudomonas auriginosa was identified in 2 (15.4\%) children. Dynamics of CRP showed that in patients with complicated course of AHO it had the bimodal character.

Initial treatment started with cefepime in all patients. At follow up, taking into account the microbial etiology the antibiotics were changed. Despite the intensive care 2 (15.4\%) children died due to the severe respiratory insufficient: one patient with belobar multi-resistant St. aureus pneumonia and one patient with AHO and pulmonary disorders caused by P. auriginosa. Thus, the CRP is feasible marker of the development of pulmonary complications in patients with AHO.

\section{2}

\section{Komplikationen der Tubularized-Incised- Plate (TIP) Harnröhrenplastik}

\section{Arneitz, A. Gössler, G. Fasching}

Kinder- und Jugendchirurgie Klinikum Klagenfurt am Wörthersee, Klagenfurt, Austria

Grundlagen. Ziel dieser Arbeit war die retrospektive Analyse der tubularized incised plate Harnröhrenplastik mit und ohne Rekonstruktion der Vorhaut sowie die Erhebung aller postoperativ aufgetretenen Komplikationen. Bei Vorliegen einer hypoplastischen Urethra wurde diese in die Harnröhrenrekonstruktion integriert.

Methodik. Es wurden 64 konsekutive Patienten mit distalen $(n=54)$, penilen $(n=8)$ und penoskrotalen $(n=2)$ Hypospadien identifiziert, die sich zwischen November 2000 und September 2007 einer TIP Harnröhrenplastik unterzogen hatten (Durchschnittsalter: 2,5 Jahre | Median: 2,0 | Spanne: 8 bis 176 Monate). Bei 33 Patienten (52\%) wurde die Vorhaut primär rekonstruiert, in 19 Fällen (30\%) lag eine dünne distale Urethra vor, die in die Rekonstruktion integriert wurde. Die Daten der Krankengeschichten wurden in allen Fällen ausgewertet und 55 Patienten (86 Prozent) durchschnittlich 4,4 Jahre (Median 4,0 | Spanne: 1-8) nach der Operation klinisch und funktionell mittels Uroflowmetrie nachuntersucht.

Ergebnisse. Zusammengefasst kam es in sechs Fällen (9\%) zu urethrokutanen, bei drei Patienten kam es $\mathrm{zu}$ einer Glansdehiszenz (5\%). Bei keinem Patienten wurde eine Stenose festgestellt. Nach Vorhautrekonstruktion zeigte sich eine signifikant erhöhte Rate an Harnröhrenfisteln $(p=0,012)$. In zwei Fällen kam es zur Vorhautdehiszenz (6\%) und in drei zu einer Vorhautlücke (9\%). Die Integration einer hypoplastischen Urethra in die Harnröhrenrekonstruktion führte $\mathrm{zu}$ einer signifikant gesteigerten Komplikationsrate $(p=0,021)$.

Schlussfolgerungen. Die tubularized incised plate Harnröhrenplastik ist ein einzeitiger Eingriff für distale und proximale Hypospadien mit geringer Komplikations- 
rate. Die hohen Zufriedenheitswerte beruhen auf guten kosmetischen Ergebnissen mit einem vertikal orientierten, schlitzförmigen und normal aussehenden Meatus an der Glansspitze sowie auf einer gut funktionierenden Neourethra.

Das generelle Erscheinungsbild wurde nach Rekonstruktion der Vorhaut signifikant besser bewertet, jedoch auf Kosten einer signifikant erhöhten Harnröhrenfistelrate. Die Vorteile der Verwendung einer distal dünnen Harnröhre haben sich nicht bestätigt.

\section{3}

\section{Hypertrophe Pylorusstenose: Der Einfluss von gastrointestinalen Viruserkrankungen bei postoperativem Erbrechen nach Pyloromyotomie}

\section{A. K. Saxena, T. Peschaut, J. Schalamon, M. E. Höllwarth}

Universitätsklinik für Kinder- und Jugendchirurgie Graz, Graz, Austria

Grundlagen. Bei postoperativem Erbrechen nach Pyloromyotomie muss eine inkomplette Spaltung der Muskulatur in Betracht gezogen werden. Ziel der Studie ist es, mögliche andere Ursachen für postoperatives Erbrechen nach Pyloromyotomie zu identifizieren.

Methodik. Wir inkludierten alle Säuglinge, die in einem 6-Jahres-Intervall (2004-2010) auf Grund einer hypertrophen Pylorusstenose operiert wurden. Im Falle von postoperativem Erbrechen $>3$ Tage wurden folgende Punkte analysiert: (1) mögliche Ursache, (2) Ergebnisse einer Reoperation, (3) postoperatives Ernährungsregime (langsam/fast track). Sowohl PatientInnen mit offener (OM) und laparoskopischer Myotomie (LM) wurden inkludiert.

Ergebnisse. 54 PatientInnen (23 OM, 31 LM) wurden inkludiert, $13 \%(n=7)$ zeigten rezidivierendes postoperatives Erbrechen. 6 von diesen 7 Säuglingen wurden reoperiert. Bei 3 von 4 Kindern nach initialer LM konnte kein Rezidiv gefunden werden, nur in einem Fall handelte es sich um eine inkomplette Myotomie. Bei 2 Kindern mit $\mathrm{OM}$ wurden ein intraoperativer Normalbefund und eine inkomplette Myotomie gefunden. Bei allen reoperierten Säuglingen mit Normalbefund wurde ein Virusinfekt diagnostiziert. Die Aufenthaltsdauer im Krankenhaus stand in keinem Zusammenhang mit dem postoperativen Ernährungsregime.

Schlussfolgerungen. Postoperatives Erbrechen nach Pyloromyotomie steht in vielen Fällen mit gastrointestinalen Viruserkrankungen in Zusammenhang. Weder die OP-Technik noch das Ernährungsregime hatte einen signifikanten Einfluss auf die Reoperationsrate oder die Aufenthaltsdauer.

\section{4}

Komplizierter Krankheitsverlauf bei einem Kind mit Morbus Hirschsprung Fallbericht

\section{S. Deluggi, G. Schimpl}

Universitätsklinik für Kinder- und Jugendchirurgie, Salzburg, Austria

Grundlagen. Die Therapie der Wahl des Mb. Hirschsprung ist die Resektion des aganglionären Darmabschnittes. Zu den häufigsten postoperativen Komplikationen zählen Obstipation, Stenosen, Fisteln und die prognostisch schlechte Enterocolitis. Nur selten kommt es als Folge ausgedehnter Resektionen zu einem Kurzdarmsyndrom.

Methodik und Ergebnisse. Wir berichten über einen Patienten der auf Grund eines kurzen Mb. Hirschsprungs einen schweren neonatalen Ileus und Enterocolitis entwickelte. Er wurde primär mit einem Ileostoma versorgt. Als Folge der Enterocolitis kam es zu ausgedehnten Schleimhautnekrosen des Dünn- und Dickdarmes. Neben der transanalen Resektion des Colosigmoids erfolgten mehrere ausgedehnte Dünn- und Dickdarmresektionen. Letztlich konnten nur ein Meter Jejunum und dreißig cm Colon erhalten werden. Aufgrund der entzündlichen Schleimhautveränderungen im betroffenen Gebiet entwickelte der Patient eine Eiweißverlustenteropathie mit sekundärem Immunglobulin-Mangel. Letztlich musste erneut wegen einer Anastomosenstenose und einem chronischen Dünndarmileus operiert werden; die Stenose wurde reseziert und eine Kathetercolostomie eingesetzt. Über den gesamten Zeitraum musste ein Drittel der Kalorien als parenteraler Ernährung zugeführt werden. Mittlerweile nimmt der Patient bei gleichbleibender parenteraler Ernährung gut an Gewicht zu. Problematisch ist nach wie vor die reduzierte Wasserrückresorptionsleistung.

Schlussfolgerungen. Selten kommt es beim Morbus Hirschsprung zu einem derart komplizierten Verlauf. Vordergründig ist, die unbehinderte Darmpassage wieder herzustellen. Dann kann eine optimale Adaptation an die Kurzdarmproblematik erfolgen.

\section{5}

\section{Complications after laparoscopic hernia repair in children}

\section{A. Dvorakevich ${ }^{1}$, A. Pereyaslov ${ }^{2}$, J. Kolivoshka ${ }^{2}$ \\ ${ }^{1}$ Regional Children's Hospital "OHMATDYT", Lviv, Ukraine;}

${ }^{2}$ Medical University, Lviv, Ukraine

Since 2008 in Lviv Regional Children's Hospital "OHMATDYT" we have been performing video laparo- 
scopic surgeries for congenital inguinal hernia by PIRS (percutaneous internal ring suturing) method. We operated 146 children.

Out of 146 patients in every 5th patient was found signs of contralateral inguinal hernia that were not apparent earlier, in such cases surgery was done on both sides. Intraoperative and in remote postoperative period in 21 (14.4\%) children the complications were noted. Intraoperative bleeding due to trauma of branches of hypogastric vessels were noted in 2 patients and hematoma of parietal peritoneum - in 12 children. Hernia recurrence was noted in 4 patients, appearance of hydrocele - in 2 children, and twist and necrosis of a loop of small intestine was in one child. Most of intraoperative complications appeared in the beginning of our use of PIRS method. The hernia recurrence determined by the not tight suturing of internal ring and was corrected by classical Duhamel hernioplasty. Reason for twist and necrosis of a loop of small intestine in one boy was strangulated intestinal obstruction, due to torsion of the loop of ileum around omentum which was captured inside not tightly closed internal inguinal ring.

\section{6}

\section{Laparoskopische Revision eines Haemoperitoneums nach laparoskopischer Appendektomie bei Willebrand-Jürgens Syndrom}

\section{A. Gössler, K. Krafka, G. Fasching}

Abteilung für Kinder- und Jugendchirurgie, Klinikum Klagenfurt am Wörthersee, Klagenfurt, Austria

Grundlagen. Beim Morbus von Willebrand-Jürgens handelt es sich um eine autosomal-dominante Erkrankung, bei der der makromolekülare Anteil des Faktors VIII, der für Trombozytenadhäsion verantwortlich ist, eine mangelhafte Funktion zeigt. Leitsymptom ist eine verlängerte Blutungszeit. Die leichten Formen werden manchmal erst bei einem operativen Eingriff erkannt. Zur Substitution des Typ I dient DDAVP (Minitrin), das Faktor VIII und von Willebrand Faktor aus körpereigenen Depots freisetzt.

Methodik und Ergebnisse. 13 jährige Patientin mit rezidivierenden Bauchschmerzen und Appendizitissymptomatik bei bekannter von-Willebrand Krankheit Typ I. Die präoperative Laboruntersuchung zeigte eine PTT von 35 sec. $(25-36,5)$ und Faktor VIII mit $63 \%$ (70-150) geringgradig herabgesetzt. Das Blutbild war im Normbereich. Die laparoskopische Appendektomie wurde wegen Appendizitis-Symptomatik nach einer Vorbereitung mit Minitrin laparoskopisch durchgeführt. Der Eingriff verlief problemlos ohne perioperative Blutung, Die Arbeitstrokare wurden unter Sicht entfernt. Histologisch fand sich eine katarrhalische Appendizitis mit Schleimhauterosion. Postoperativ wurde die Patientin auf der Intensivstation überwacht. Am ersten postoperativen Tag wurde klinisch und sonographisch ein Hämoperitoneum, sowie später ein ausgedehntes subkutanes Hämatom im linken Hypogastrium festgestellt.

Es kam zum Abfall des Hämatokrits auf $18 \%$ (35-44), $\mathrm{Hb}$ auf 6,1 g/dl $(12-15,4)$ und Erythrozyten auf 2,27 $\times 10^{6}$ $(4,8-12)$. Faktor VIII war nach der Substitution mit $168 \%$ erhöht und die Gerinnung im Normbereich. Nach der Gabe von Erythrozytenkonzentraten wurde die Indikation zur laparoskopischen Revision am 2. postoperativen Tag gestellt. Während der Laparoskopie wurde das flüssige Blut, sowie die vielen Koageln ausgeräumt, intraperitoneal kein blutendes Gefäß festgestellt. Als Blutungsquelle wurde eine bereits nur mehr geringgradige subfasziale Blutung aus der Trokarwunde im linken Mesogastrium identifiziert. Die Wunde wurde erweitert und die Blutung mit bipolarer Pinzette und Durchstechungsligatur behandelt. Der weitere postoperative Verlauf war komplikationslos.

Schlussfolgerungen. Eine kompensierte von Willebrand-Jürgens Krankheit präsentiert keine Kontraindikation zu einer Laparoskopie. Nachdem die Trokare beim ersten Engriff unter Sicht entfernt wurden, konnte man die Komplikation nicht beeinflussen.

Im Rückblick stellt sich die Frage, ob die Laparoskopie beim primären Eingriff indiziert war oder ein höheres Risiko darstellte. Andererseits war die laparoskopische Revision trotz der schwierigen Bedingungen beim ausgedehnten Hämoperitoneum und der von Willebrand Krankheit erfolgreich und komplikationslos.

\section{Österreichische Gesellschaft für Adipositaschirurgie: Chirurgische Technik}

\section{7}

\section{Gute Erfahrungen mit der Sleeve Gastrektomie als einziges geplantes bariatrisches Verfahren}

\section{Kees-Belyus, C. Sperker, M. Schermann, A. Abrahim, A. Landsiedl, R. Roka}

Rudolfstiftung Vienna, Department of Surgery, Vienna, Austria

Grundlagen. Seit 1,5 Jahren wird an der Krankenanstalt Rudolfstiftung Wien ein Konzept der Single Step Sleeve Gastrektomie verfolgt. Die Sleeve Gastrektomie ist bisher als Ersteingriff für super-obese Patienten etabliert. Der Vortrag beschreibt das Gesamtkonzept dieser Operation als einzigen geplanten bariatrischen Eingriff für ein erweitertes Patientengut. 
Methodik. In den letzten 18 Monaten wurden 83 Patienten (59 Frauen, 24 Männer) einer Single Step Sleeve Gastrektomie unterzogen. Der mediane BMI betrug vor der Operation $47 \mathrm{~kg} / \mathrm{m}^{2}$ (range 38-74).

Ergebnisse. Die Patienten, die für eine Single Step Sleeve Gastrektomie in Frage kommen, zeichnen sich vor allem durch junges Alter und fehlende Komorbidität aus. Die begleitende Ernährungstherapie hat, wie auch bei malabsorptiven Eingriffen, einen besonderen Stellenwert.

Schlussfolgerungen. Bereits nach 6 bzw. 9 Monaten erreichten die Patienten einen medianen Übergewichtsverlust von 57 Prozent (range 26-86) bzw. 71 Prozent (range 39-85). Der postoperative stationäre Aufenthalt betrug median 4 Tage (range 3-7). Als Komplikationen traten bei 2 Patienten Nachblutungen auf, die aber laparoskopisch gut beherrschbar waren. Im Rahmen der Nachsorge berichteten 6 Patienten von milden Reflux Symptomen. Die Single Step Sleeve Gastrektomie findet sowohl medizinisch als auch von Patientenseite hohe Akzeptanz.

\section{8}

\section{Jahres Ergebnisse Single Step Sleeve Gastrektomie Neuer Goldstandard der bariatrischen Chirurgie?}

\section{A. Abrahim, M. Kees, S. Ali-Abdullah, A. Landsiedl, M. Schermann, C. Sperker, R. Roka}

Krankenanstalt Rudolfstiftung, Wien, Austria

Grundlagen. Die laparoskopische Sleeve-Gastrektomie stellt ein Operationsverfahren dar, das als First Step Sleeve Eingriff für „super obese“ Patienten entwickelt wurde. In weitere Folge sollte nach bereits begonnener Gewichtsreduktion ein laparoskopischer Magenbypass angelegt werden. Der Großteil der Patienten jedoch war mit der bereits stattgefunden Gewichtsabnahme so zufrieden, dass sie von einem zweiten Eingriff Abstand nahmen.

Methodik. Alle Patienten aus den Jahren 2003-2006, die sich einzig einer Sleeve-Gastrektomie unterzogen haben, wurden untersucht. Maximaler Gewichtsverlust, aktuelles Gewicht, Refluxsymptomatik, die Entwicklung vorhandener Komorbiditäten, sowie das subjektive Wohlbefinden wurden berücksichtigt.

Schlussfolgerungen. Unsere Ergebnisse bestätigen, dass ein Single Step Sleeve Verfahren als bariatrische Operation eine ausreichende Gewichtsabnahme mit all den assoziierten Vorteilen erzielen kann. „Weight regain“ kann in Einzelfällen beobachtet werden, ist jedoch nicht primär die Folge fehlender Langzeitwirkung der SleeveGastrektomie. Weiters zeigt sich ein hohes subjektives Wohlbefinden. Weitere Studien sind notwendig, um den bisherigen Stellenwert der Sleeve Gastrektomie als „First Step“ neu zu evaluieren.

\section{9}

Single center experience with sleeve gastrectomy: short-term results and technical considerations of 44 cases

\section{A. Weißenbacher, A. Perathoner, E. Laimer, G. MühImann, J. Pratschke, R. Mittermair}

Department of Visceral, Transplant and Thoracic Surgery, Center of Operative Medicine, Medical Unive, Innsbruck, Austria

Background. Bariatric surgery has been established as the best option of treatment for morbid obesity. Sleeve gastrectomy provides adequate loss of weight with little morbidity. The aim of this study was to report our experience with sleeve gastrectomy.

Methods. Between 04/2006 and 11/2010 sleeve gastrectomy was performed in 44 consecutive patients. All operations were performed by a technique with attention to avoiding strictures at the incisura angularis and stapling close to the esophagus at the angle of His. A retrospective analysis was conducted to determine the results.

Results. Thirty-two women and 12 men received a sleeve gastrectomy. Eight patients underwent laparoscopic gastric banding before; simultaneous band removal was done twice. In 40 cases the sleeve gastrectomy was performed laparoscopically, in which 1 conversion to an open procedure was necessary. Four patients underwent an open sleeve gastrectomy primarily. After a mean follow-up of 13.61 ( $S D \pm 13.87$ ) months the change in BMI and body weight was $7.658 \mathrm{~kg} / \mathrm{m}^{2} \pm 1.903(p=0.0001)$ and $21.69 \mathrm{~kg} \pm 6.379(p=0.0011)$.

Conclusions. Sleeve gastrectomy leads to stable weight loss. Benefits of sleeve gastrectomy include low rate of complications, the avoidance of foreign material and the maintenance of normal gastro-intestinal continuity.

\section{0}

\section{Single incision laparoscopic sleeve gastrectomy: tips and tricks}

\section{R. Mittermair, S. Scheidl, J. Pratschke, G. Mühlmann}

Department of General and Transplant Surgery, Innsbruck, Austria

Background. Bariatric surgery has been established as the best option of treatment for morbid obesity. In recent years single-incision laparoscopic surgery (SILS) has emerged as another modality of carrying out the bariatric procedures. While SILS represents an advance, its application in morbid obesity at present is limited. 
Methods. Patients who underwent single-incision laparoscopic sleeve gastrectomy at our instituation were analyzed. A transumbilical incision was used. Laparoscopic sleeve gastrectomy was performed in a standard fashion using a 36 French bougie.

Results. There were no intra- and postoperative complications.The patients were highly satisfied with the scar. We report here some tips and tricks to perform an easily feasible SILS sleeve gastrectomy.

Conclusions. Because of abundant visceral and subcutaneous fat and multiple comorbidities in morbid obesity, it is more challenging for surgeons to perform the procedures with SILS. It is clear that extensive development of new instruments and technical aspects of these procedures as well as randomized studies to compare them with traditional laparoscopy are essential before these procedures can be utilized in day-to-day clinical practice.

\section{1}

\section{Therapie morbider Adipositas - Renaissance des Magenballons?}

\section{A. Abrahim, C. Sperker, M. Kees, S. Ali-Abdullah, A. Landsiedl, M. Schermann, R. Roka}

Krankenanstalt Rudolfstiftung, Wien, Austria

Grundlagen. In der metabolischen Chirurgie haben sich die laparoskopische Sleeve-Gastrektomie und der Magenbypass weitgehend durchgesetzt. Bei zahlreichen Patienten erscheint ein derartiges Vorgehen jedoch als „Overtreatment“ oder sie lehnen einen Eingriff auf Grund der Invasivität bzw. Irreversibilität grundsätzlich ab. In solchen Fällen präsentiert sich der neu entwickelte adjustierbare Magenballon als vielversprechende Alternative.

Methodik. Eine 49 jährige Patientin zeigte eine deutliche Gewichtszunahme (BMI 30) unter Nikotinabstinenz. Alle konservativen Versuche, ihr Normalgewicht zu halten, schlugen fehl. Nach entsprechender Evaluierung ihrer Situation wurde ihr ein neu entwickelter adjustierbarer Magenballon im Rahmen einer herkömmlichen Gastroskopie implantiert. Die Patientin wurde über den Zeitraum von 6 Monaten kontinuierlich einem Follow-up unterzogen.

Schlussfolgerungen. Der adjustierbare Magenballon stellt eine effiziente, minimal-invasive und kostengünstige Alternative $\mathrm{zu}$ den bereits etablierten chirurgischen Verfahren zur Therapie von Adipositas dar. Die Renaissance des Magenballons als Therapiekonzept für ein selektioniertes Patientengut kann nicht geleugnet werden.

\section{2}

\section{Langzeitkomplikationen beim laparosopkischen Magenbypass}

\section{Sperker, A. Abrahim, S. Ali-Abdullah, M. Kees, A. Landsiedl, M. Schermann, R. Roka}

Krankenanstalt Rudolfstiftung, Wien, Austria

Grundlagen. Adipositas ist eine sich in zunehmenden Ausmaß ausbreitende Zivilisationserkrankung, wobei viele dieser Patienten den letzten Ausweg in der bariatrischen Chirurgie sehen. Dabei gilt es jedoch nicht nur die Anforderungen des Eingriffes selbst sowie der unmittelbaren Nachsorge zu bewältigen, sondern auch mit den Langzeitkomplikationen und deren Managment $\mathrm{zu}$ rechnen.

Methodik. Es werden Case Reports von Patienten vorgestellt, welche mit einer Langzeitkomplikation nach Anlage eines laparoskopischen Magenbypasses aufgrund einer morbiden Adipositas im Jahr 2010 an der chirurgischen Abteilung der Krankenanstalt Rudolfstiftung behandelt wurden.

Ergebnisse. Unter den Langzeitkomplikationen fanden sich Bridenileus, Dünndarmtorsionen, Blutungen aus dem Restmagen, penetrierende Anastomosenulcera, Anastomosenstenosen sowie die gesamte Erscheinungsbreite der Mangelernährung bis hin zur transfusionspflichtigen Eisenmangelanämie.

Schlussfolgerungen. Anbetracht der zunehmenden Operationszahlen und des häufig sehr jungen Alters der Patienten ist eine besonders strenge Indikationsstellung in der bariatrischen Chirurgie notwendig.

\section{3}

\section{Bariatric surgery effects addiction for overeating and eating disorders}

\author{
K. Miller ${ }^{1}$, E. Ardelt-Gattinger ${ }^{2}$, S. Ring ${ }^{3}$, \\ D. Weghuber ${ }^{4}$, M. MeindI ${ }^{5}$
}

${ }^{1}$ Chirurgie, Hallein, Austria; ${ }^{2}$ Psychologisches Institut Universität, Salzburg, Austria; ${ }^{3}$ Sportinstitut Universität, Salzburg, Austria; ${ }^{4}$ Kinderklinik Privatmedizinische Universität, Salzburg, Austria; ${ }^{5}$ Psychologie Universität, Salzburg, Austria

Grundlagen. Chirurgische Adipositastherapie erzielt die für die Vermeidung oder Besserung von lebensgefährlichen Komorbiditäten nötige dauerhafte Gewichtsabnahme. Über Veränderungen der psychologischen Ätiologie- und Risikofaktoren ist wenig bekannt.

Methodik. In der vorliegende Studie wurde die Veränderung von psychischen Symptomen, die als Ätiologiefaktoren der Adipositas in Frage kommen bzw. stark belasten an 60 PatientInnen prä $(24 \mathrm{~m} / 36$ w, 18-71, BMI 
$\mathrm{M}=44,95, \mathrm{SD}=6,91$ ) und post (BMI $\mathrm{M}=33,92$, SD $7,23)$ Bypass bzw. Magenband mit Hilfe des neuen Testsystems AD-EVA überprüft. Alle PatientInnen weisen zufriedenstellende Testgütekriterien auf und enthalten Normwerte für die einzelnen BMI Gruppen, die an einer repräsentativen österreichischen Stichprobe ermittelt wurden.

Ergebnisse. Die Studie zeigte, dass sich die Variablen "Sucht" ( $t=11,15, p<0,000)$ und „Binge Eating Disorder“ $(t=2,13, p<0,05)$ über alle PatientInnen hinweg signifikant in die gewünschte Richtung verändern, sich aber in Abhängigkeit von der Methode Bypass versus Band hoch signifikante $(p<0,01)$ Unterschiede ergeben

Schlussfolgerungen. Indikation zu Magenband versus Bypass sowie Vorschriften über präoperative Psychotherapien sind am Hintergrund jener Ergebnisse, die mit rezenter Literatur konform sind, differenzierter als bisher zu betrachten.

\section{4}

\section{Laparoscopy in the morbidly obese}

\section{N. Loibner-Ott, T. Weber, C. Harrer, S. Kriwanek}

SMZ-Ost, Donauspital, Vienna, Austria

Background. Laparoscopic bariatric surgery requires abdominal insufflation with increased intraabdominal pressure up to $18 \mathrm{mmHg}$.

Methods. We setup a study to investigate the intraabdominal pressure at the beginning of the insufflation as well as the volume of $\mathrm{CO}_{2}$ needed to achieve a pneumoperitoneum of $12 \mathrm{mmHg}$ and $14 \mathrm{mmHg}$. Operating time and size of the left liver lobe were documented, and the surgeon was asked to give a personal score of the difficulty of the procedure.

Results. Physiologically, morbidly obese patients demonstrate 2 - 3-fold higher itraabdominal pressures compared to non obese persons. The level of difficulty of the procedure is influenced by several factors including the size of the left liver lobe and has an effect on the operating time.

Conclusions. The results of the study will give information how intraabdominal pressures and volume of insufflated $\mathrm{CO}_{2}$, as well as the size of the left liver lobe have an effect on difficulty and duration of procedures. An extended pre- (ultrasonography) and intraoperative (pressure and volume measurement) assessment could be valuable for the planning of bariatric procedures especially in high risk patients.

\section{5}

\section{Stenose der Gastrojejunostomie nach Roux-Y-Magenbypass}

\section{P. Beckerhinn, S. Schöppl, A. Grill, D. Cadariu, C. Fenz, F. Hoffer}

Chirurgie, Hollabrunn, Austria

Grundlagen. Die Stenose an der Gastrojejunostomie nach Roux-Y-Magenbypass (RYGBP) ist eine relativ häufige Komplikation, die in den ersten postoperativen Monaten auftreten kann und mittels Ballondilatation unter Sedierung mit Midazolam oder Propofol therapiert wird. Das Ziel dieser Studie war, die Stenoserate an unserer Abteilung nach mehr als 600 Eingriffen mit einem $21 \mathrm{~mm}$-Ringstapler $\mathrm{zu}$ untersuchen um die eventuelle Notwendigkeit eines Staplerwechsels auf $25 \mathrm{~mm}$ zu überlegen.

Methodik. Zwischen Juni 2004 und Dezember 2010 wurden 642 (w: 524, m: 118) RYGBP-Operationen durchgeführt (592 laparoskopisch, 50 offen). Darunter waren 121 Revisionseingriffe nach Magenband, Sleeveresektion, VBO oder Magenschrittmacher. In 641 Fällen wurde ein 21-mm Ringstapler verwendet, einmal ein 25-mm Stapler. Der durchschnittliche BMI zum Operationszeitpunkt betrug $45 \mathrm{~kg} / \mathrm{m}^{2}$ (19 bis 72), das Durchschnittsalter war 41 Jahre (17 bis 69$)$.

Ergebnisse. 21 Patienten entwickelten im ersten postoperativen Jahr eine Stenose an der Gastrojejunostomie $(3,29 \%) .20$ mal wurde die Stenose erfolgreich dilatiert, eine komplette Stenose musste am ersten postoperativen Tag neu angelegt werden. Es traten keine Komplikationen im Rahmen der Dilatationen auf.

Schlussfolgerungen. Unsere Studie zeigt, dass die Gastrojejunostomie mittels 21-mm Stapler sicher durchgeführt werden kann. Die Stenoserate ist akzeptabel niedrig. Die Therapie mittels Ballondilatation ist sicher und wird von den Patienten gut toleriert.

\section{6}

\section{Petersen Hernie nach laparoskopischem antecolischen Roux-Y Magenbypass}

\section{S. Sattler, O. Riedl, R. Ciovica, W. Lechner, M. Gadenstätter}

Chirurgische Abteilung des LK Krems, Krems, Austria

Die Petersen Hernie ist nach laparoskopischem antecolischen Roux-Y Magenbypass eine seltene aber potentiell gefährliche Komplikation mit einer Inzidenz von bis zu 6,2\%. An unserer Abteilung wurde bis Ende Juni 2010 bei 282 Patienten der Mesenterialschlitz am Petersen Space im Konsens mit anderen Abteilungen nicht verschlossen. 
In diesem Patientenkollektiv sind 4 Fälle von Petersen Hernien aufgetreten. Alle Patienten wurden operiert, in einem Fall konnte die Herniation laparoskopisch behoben werden, in zwei Fällen wurde eine Laparotomie durchgeführt und in einem weiteren Fall wurde eine offene Revision mit Dünndarmteilresektion notwendig.

Die frühzeitige Diagnose ist aufgrund oft unauffälliger CT-Befunde schwierig.

Selbst bei blander Bildgebung sollte bei einem antecolischem Roux-Y Magenbypass und postprandialen Beschwerden eine diagnostische Laparoskopie durchgeführt werden. Bei jedem antecolischen Roux-Y Bypass ist auf einen sicheren Verschluss des Petersen Space zu achten.

\section{Hauptsitzung - Komplikationsmanagement 4: Viszeralchirurgie}

\section{2}

\section{Interdisziplinäres Management schwerwiegender postoperativer Komplikationen nach Pankreasresektion}

T. Hoblaj ${ }^{1}$, S. Stättner ${ }^{1}$, M. Horvath ${ }^{1}$, F. Karnel ${ }^{2}$, G. Strau ${ }^{2}$, W. Hackl ${ }^{3}$, J. Karner ${ }^{1}$

${ }^{1}$ SMZ Süd KFJ, Chirurgische Abteilung, Wien, Austria; ${ }^{2} \mathrm{SMZ}$ Süd KFJ, Zentralröntgeninstitut, Wien, Austria; ${ }^{3} \mathrm{SMZ}$ Süd KFJ, Abteilung für Anästhesie und operative Intensivmedizin, Wien, Austria

Grundlagen. Die Pankreaschirurgie ist nach wie vor mit einer hohen peri- und postoperativen Morbidität verbunden, postoperative Komplikationen treten in 1 bis $9 \%$ auf. Die schwerwiegendsten Komplikationen mit einer Letalität von 11 bis $38 \%$ stellen frühe oder späte postoperative Blutungen dar. Die sichere Diagnostik und eine rasche, adäquate Therapie erfordert eine konsequente interdisziplinäre Zusammenarbeit.

Methodik. Anhand eines Fallberichtes wird das Management von zwei späten postoperativen Major-Komplikationen (Grad IV nach Clavien-Dindo) dargestellt.

Der Fall: Vier Wochen nach Whipple-Operation wegen Pankreaskopfkarzinom kommt es bei einer 66jährigen Patientin zu einer akuten Arrosionsblutung aus der A. hepatica propria, sowie durch einen akuten Verschluß $\operatorname{der} A$. hepatica sinistra zur konsekutiven Nekrose des linken Leberlappens. Nach Diagnose mittels MDCT wird die Patientin intensivmedizinisch betreut und kreislaufstabilisiert, die Blutung interventionell-radiologisch durch Stentimplantantion gestoppt und der nekrotische Leberanteil im Sinne einer linksseitigen Hemihepatektomie chirurgisch entfernt. Drei
Wochen später kann die Patientin in gutem Allgemeinzustand entlassen werden.

Schlussfolgerungen. Die direkte und klare Kommunikation zwischen zusammenarbeitenden Fachrichtungen wie Radiologie, Intensivmedizin und Chirurgie schafft die Rahmenbedingungen für die Behandlung postoperativer Komplikationen. Für eine erfolgreiche Bewältigung ist einerseits die rasche Verfügbarkeit diagnostischer Ressourcen sowie erfahrenen Fachpersonals, andererseits ein Informationsaustausch ohne Umwege erforderlich. Dies ist in kleineren Einheiten mit guter Kommunikationskultur der Fall.

\section{3}

\section{Vergleich der Inzidenz und Morbidität von Pankreasfisteln nach Duodenopankreatektomie und Linksresektion}

\section{O. Gangl ${ }^{1}$, U. Fröschl ${ }^{1}$, T. Sautner ${ }^{2}$, R. Függer ${ }^{1}$}

${ }^{1}$ Krankenhaus der Elisabethinen/Chirurgische Abteilung, Linz, Austria; ${ }^{2}$ Krankenhaus der Elisabethinen/Chirurgische Abteilung, Wien, Austria

Grundlagen. Nach Pankreaslinksresektionen wird die Fistelrate, nach Rechtsresektionen die Blutungsrate höher angegeben. Ziel der Studie war der Vergleich der Fistelrate am eigenen Krankengut und die Analyse des Komplikationsmanagements.

Methodik. In 10 Jahren (2001-2010) erfolgten $n=171$ Pankreas Rechtsresektionen ( $n=155$ pyloruserhaltende Duodenopankreatektomien; $n=16$ Kausch Whipple Operationen) und $n=56$ Pankreas Linksresektionen.

Ergebnisse. Nach Pankreaslinksresektion besteht eine Fistelrate von $8,9 \%$ (5/56), nach Rechtsresektion von $8,2 \%(14 / 171)$ und kein signifikanter Unterschied im Gruppenvergleich mittels fisher exact test (2-tailed $p=0,788$ ). Ebenso waren bei der Blutung (Rechtsresektion $5,3 \% \quad(9 / 171) \quad v s$. Linksresektionen $1,8 \% \quad(1 / 56)$ $p=0,457)$ und der Mortalität $(2,9 \%$ (5/171) vs. $0 \%$ $(0 / 56) \quad p=0,336)$ keine signifikanten Unterschiede. Das Komplikationsmanagement der 5 Fisteln nach Linksresektion (keine operative Revision; 2 sonographisch gezielte Punktionen) unterschied sich von dem der 14 Fisteln nach Rechtsresektion (6 operative Revisionen; 4 CT gezielte Punktionen; 2 Angiographien). Der Schweregrad der Fisteln nach der Definition von DeOliveira et. al. Ann. Surg. 2006; PMID 17122618 zeigt nach Rechtsresektion (II $n=2$; IIIa $n=6$; IVa $n=3$; IVb $n=1 ; \mathrm{V} n=2$ ) und Linksresektion (II $n=3$; IIIa $n=2$ ) einen grenzwertig signifikanten Unterschied der Inzidenz von Grad II Fisteln $(p=0,084)$.

Schlussfolgerungen. Der Vergleich Pankreasrechts$v s$. Linksresektion zeigt keinen signifikanten Unterschied hinsichtlich Fistel- und Blutungsrate oder Schweregrad der Fisteln. 


\section{4}

\section{Behandlungsalgorithmus interventioneller Ultraschall-gestützter Prozeduren bei Pancreaspseudozyste, Abszess \& infizierter Nekrose - Progressionsreport einer prospektiven klinischen "Single- center"-Observationsstudie}

\author{
U. Will ${ }^{1}$, C. Wanzar ${ }^{1}$, F. Meyer ${ }^{2}$ \\ ${ }^{1} \mathrm{SRH}$-Waldklinikum, Klinik für Gastroenterologie, Gera, \\ Germany; ${ }^{2}$ Universitätsklinikum A.ö.R., Klinik für Allgemein-, \\ Viszeral- \& Gefäßchirurgie, Magdeburg, Germany
}

Grundlagen. Machbarkeit/ „Outcome“ der Ultraschall-/ EUS-geführten Drainage von Pancreaspseudozysten/ -abszessen sowie des endoskopischen Debridements infizierter Pancreasnekrosen in systematischer prospektiver „Single-center“-Observationsstudie (Fallserie) mittels Computer-basiertem Datenregister. Machbarkeit wurde durch Erfolgs-/Komplikationsrate charakterisiert, Outcome durch Morbidität, Mortalität \& Rezidivrate.

Ergebnisse. Vom 23.03.2002 bis 31.12.2008 wurden 147 Patienten (Geschlechtsverhältnis: Frauen/Männer 49/98[1:2,0]) mit symptomatischen nichtinfizierten Pancreaspseudozysten $(n=32)$, -abszessen $(n=81)$ \& infizierten-nekrosen $(n=34)$ erfasst. Die technische Erfolgsrate bei der Ultraschall-gestützten externen \& EUS-geführten transmuralen Drainage war $100 \%$ bzw. 97\% (transpapilläre [ERP-gestützte] Drainage: 92,1\%). Während die akute interventionsbezogene Komplikationsrate der externen Drainage 3,7\% ausmachte, lag diese Rate bei transmuraler/transpapillärer Drainage bei 9,6\%/0. Späte Komplikationen ( $>24 \mathrm{~h}$ ) wurden bei $6,4 \%$ der Patienten nach transpapillärer Drainage beobachtet (externe/transmurale Drainage: 5,6\%/19,1\%). Komplikationen in 5Fällen (Blutung: $n=3 /$ Perforation: $n=1$ Prothesendislolation mit Ileumperforation: $n=1$ ) mussten operiert werden. Nach mittlerem Follow-up-Zeitraum von 20,7; 20,9 bzw. 19,4 Monaten betrug die definitive therapeutische Erfolgsrate durchschnittlich 96,2\% für die 3 Diagnosen Pseudozyste (96,9\%), Abszess (97,5\%) \& Nekrose $(94,1 \%$ - mittlere Rezidivrate: $15,4 \%$; Gesamtmortalität: $0,7 \%$, jedoch nicht interventionsbezogen).

Schlussfolgerungen. Mehrheitlich erwies sich konsequentes Ultraschall- \& Endoskopie-gestütztes Management von Pancreasläsionen wie Pseudozyste, Abszess \& infizierter Nekrose mittels transpapillärer, Ultraschallgeführter externer \& EUS-geführter transmuraler Drainage als auch mittels endoskopischem Debridement als machbar \& geeignet, das auch in täglicher Routine zu favorisieren ist, da es als sicher \& effektiv in erfahrener Hand anzusehen ist.

\section{5}

Giant pseudo-liver tumor: pitfalls and complications in the management

\author{
K. Pimpl, W. Brunner, J. Schirnhofer, C. Mittermair, \\ C. Obrist, N. Waldstein, R. Frass, H. Weiss
}

Chirurgie, Salzburg, Austria

Background. Preoperative diagnosis can be made with high accuracy for tumors in the mid and upper abdomen by means of US, CT and serum tests. We describe the case of an inconclusive intra-retroperitoneal tumor.

Methods. 41 year-old male patient, suffering from a giant tumor $(12 \times 10 \mathrm{~cm})$ that was suspicious for exophytic hepatocellular carcinoma in the preoperative staging (US, CT scan). No concrete assignment to an organ was made. Serum tumor-markers and liver-function-tests were normal. Informed consent for a single port laparoscopic procedure was obtained.

Results. Preparation of the tumor could be completed without adding additional trocars. The tumor showed strong vascularisation with a small section of ingrowing duodenal serosa. Preparation was continued in open technique. Assuming the tumor being a sarcoma of the renal capsule an en-bloc exstirpation was performed. On postoperative day seven severe duodenal bleeding required emergency relaparotomy. Thereby a full-thickness resection of the duodenal wall was performed.

Histologic evaluation surprisingly revealed the diagnosis of a gastrointestinal stroma tumor with malignant potential. The interdisciplinary tumor board found the patient eligible for additive Imatinib therapy.

Conclusions. Gastrointestinal stroma tumors also may occur as a primary tumors of the omentum, mesentery or retroperitoneum.

\section{6}

Bridging the gap with an ileocolonic graft after extensive colorectal resections: long-term results

\section{B. Dauser ${ }^{1}$, S. Riss ${ }^{2}$, J. Stopfer ${ }^{1}$, F. Herbst ${ }^{1}$}

${ }^{1}$ St. John of God Hospital, Department of Surgery, Vienna, Austria; ${ }^{2}$ Department of Surgery, Medical University of Vienna, Vienna, Austria

Background. Ileocecal interposition (ICI) for first-line reconstruction after low anterior resection was introduced by von Flüe in 1994. We report our experience using this technique to bridge colonic gaps after significant loss of bowel length.

Methods. Between 1999 and 2009 the left-sided colon was too short for traditional isoperistaltic reconstruction 
in 6 patients. Reasons for extensive bowel loss were a deficient or torn marginal artery with consecutive ischemia $(n=4)$ or repeated resections for local rectal cancer recurrence $(n=2)$. An ICI was done to bridge the gap and enable restoration of the intestinal continuity.

Results. No patient died. Whenever performing a coloanal anastomosis (4/6) a loop ileostomy was raised. Ischemic colitis of the graft was observed in a male patient and was managed non-operatively. Two anastomosis-vaginal fistulas occurred and were treated successfully via transanal or transvaginal approach. All ileostomies were closed subsequently. During follow-up (median: 34 months, range 10-141) no further complications were recorded. A median Vaizey incontinence score of 6 (range: $0-14)$ was found. Average number of bowel movements per day accounted for 1.5 (range: 0.5-6).

Conclusions. ICI to maximise colon length in re-operative cases or after vascular complication is a reasonable approach resulting in good bowel function.

\section{Österreichische Gesellschaft für Gefäßchirurgie: Aktuelles aus der arteriellen und venösen Gefäßmedizin}

\section{2}

\section{Der hohe Abgang der A. tibialis posterior: Inzidenz und Konsequenz für die Chirurgie der Unterschenkelgefäße}

\section{Aspalter, A. Ugurluoglu, K. Linni, T. Hölzenbein}

Paracelsus Medizinische Privatuniversität, Department für Gefäßchirurgie, Salzburg, Austria

Grundlagen. Variationen der Unterschenkeltrifurkation werden in ca. $10 \%$ beschrieben. Die häufigste Variation ist ein hoher Abgang der A. tibialis anterior mit ca. $5 \%$. Variationen der A. tibialis posterior (ATP) sind selten und werden in $0,5-2,5 \%$ beobachtet. Das Ziel unserer Studie war es die Häufigkeit des hohen Abganges der ATP in unserem Krankengut zu untersuchen.

Methodik. Prospektive Analyse von Angiogrammen von Patienten mit peripherer Ischämie an einem universitären Zentrum für Gefäßchirurgie.

Ergebnisse. Zwischen 03/2006 und 11/2010 wurden 560 Angiogramme (360 fem-distale Bypasses, 200 periphere Katheterinterventionen) evaluiert. Davon wurde bei 12 Patienten (durchschnittliches Alter 61,9 Jahre; 10 männlich; PAVK IV: $n=5$, PAVK III: $n=2$, PAVK IIb: $n=4$, Trauma: $n=1$ ) ein hoher Abgang der ATP identifiziert (links: $n=7$; bilateral: $n=2$ ). Die primäre technische Erfolgsrate der Gefäßrekonstruktion (Fem-distaleVenen- bypass (VBP): $n=5$, komplexe Rekonstruktion der Beckenetage: $n=1$, PTA der Unterschenkelgefäße: $n=6$ ) war $80 \%$. Nach einem durchschnittlichen Beobachtungszeitraum von 11,8 Monaten waren alle Rekonstruktionen für pAVK offen. Der Patient mit Unterschenkeltrauma verlor aufgrund eines massiven Weichteiltraumas den Unterschenkel.

Schlussfolgerungen. Der hohe Abgang der ATP wurde in unserem Patientengut in 2,1\% identifiziert und ist mit der angegebenen Literatur vergleichbar. An diese seltene Variation sollte bei der Chirurgie der Unterschenkelgefäße gedacht werden.

\section{3}

\section{A feasibility study to evaluate compartment pressure using ultrasound}

\section{J. Mühlbacher ${ }^{1}$, A. Duma ${ }^{1}$, R. Pauzenberger ${ }^{2}$, R. Pikula ${ }^{2}$, S. Kapral ${ }^{1}$}

${ }^{1}$ MUW, General Hospital, Department of Anaesthesiology and General Intensive Care, Vienna, Austria; ${ }^{2}$ Department of Plastic Surgery, Innsbruck Medical University, Innsbruck, Austria

Background. Direct invasive measurement of the intramuscular pressure is the gold standard in diagnosing a compartment syndrome. This pilot study investigates the angle between the tibia bone and the muscle fascia as a surrogate parameter for the increasing pressure in the ventral compartment of the lower leg using ultrasound.

Methods. Intracompartmental pressure in ten human cadavers (20 legs) was increased stepwise from $10 \mathrm{mmHg}$ up to $100 \mathrm{mmHg}$ under continuous recording using a hydrostatic saline infusion. On standardized measure points in the transversal plain, the angle between the lateral face of the tibia bone and the expanding fascia of the anterior compartment were measured by means of real time ultrasound in $10 \mathrm{mmHg}$ steps while slowly raising the pressure.

Results. The mean measured angle enlarged from $66.6 \pm 10.6$ degrees at $10 \mathrm{mmHg}$ up to $86.1 \pm 8.8$ respectively at a $100 \mathrm{mmHg}$ level. There was positive correlation $(r=0.54, p<0.001)$ between pressure and the described angle.

Conclusions. The expansion of this measured angle represents the current pressure in the ventral muscle compartment. Under standardized measurement, this criterion is objective and repeatable. Continuous monitoring as well as the comparison of both legs may predict a developing compartment syndrome. 


\section{4}

\section{Die Ruptur eines Aneurysmas der Arteria suprarenalis als seltene Ursache eines retroperitonealen Hämatoms-Fallbericht und Literaturrecherche}

\section{B. Müller}

Hanusch Krankenhaus, Abteilung für Allgemeinund Gefäßchirugie, Wien, Austria

Aneurysmen der paarigen Äste der infradiaphragmalen Aorta sind seltene Entitäten, wobei nach unserem Wissensstand in der Literatur nur einzelne Kasuistiken zur Ruptur eines Aneurysmas der A. suprarenalis beschrieben werden.

Es wird der Fall eines 71-jährigen männlichen Patienten vorgestellt, der unter dem Verdacht einer Nierenkolik an der urologischen Abteilung unseres Hauses aufgenommen wurde. Computertomographisch bildete sich ein ausgeprägtes retroperitonales Hämatom ab, dessen Ursprung in einer Ruptur der linken Arteria suprarenalis suspiziert wurde. Die operative Versorgung, die aufgrund der Größe des Hämatoms indiziert war, bestand in einer Dissektionsresektion sowie Ligatur der Arteria suprarenalis, die destruierte Nebenniere wurde partiell reseziert. Der postoperative Verlauf war komplikationslos. Die histologische Aufarbeitung des Operationspräparates zeigte ausgeprägte arteriosklerotische Wandveränderungen im rupturierten Aneurysma.

Wird ein Aneurysma der Arteria suprarenalis als Zufallsbefund diagnostiziert, stellt sich die Frage nach seiner Therapiebedürftigkeit bzw. den therapeutischen Optionen. Der Literatur entsprechend sollte eine Polyarteritis nodosa ausgeschlossen werden. Zur Diskussion steht, ob bei vaskulären Pathologien des Retroperitoneums in Analogie zu den häufiger beschriebenen Viszeralerterienaneurysmen vorzugehen ist. Der endovaskulären Versorgung sollte zumindest bei asymptomatischen Aneurysmen sowie im Frühstadium eines Blutungsgeschehens der Vorzug gegeben werden.

\section{5}

\section{Kidney autotransplantation for renal artery aneurysm}

\author{
J. Celeiro, C. Rouden, S. Bischofberger, M. Adamina, \\ W. Nagel \\ Klinik für Chirurgie, St. Gallen, Switzerland
}

Background. The incidence of renal artery aneurysms (RAAs) goes up to $2.5 \%$ in patients with hypertension. We present a successful kidney autotransplantation as treatment of symptomatic RAA.
Methods. A 44-year-old woman was referred with severe hypertension and a history of left nephrectomy for hydronephrosis and cystic kidney disease at 1 year of age. CT identified 3 RAAs $(14-34 \mathrm{~mm})$. An interdisciplinary conference proposed surgery to prevent RAA rupture and possibly control arterial hypertension.

Results. A subcostal incision was performed to have full vascular control. Kidney preservation was initiated by controlled hypothermia and perfusion of a Celsior ${ }^{\circledR}$ solution. The RAAs, reaching up to the renal hilus, were resected ex vivo leaving short arterial branches, which were sutured into a common ostium. Kidney was autotransplanted in the left iliac fossa. Cold ischemia time was $108 \mathrm{~min}$. Postoperative diuresis and radiological controls were normal. A pyelonephritis was successfully treated with ciprofloxacin. The patient was discharged with normal blood pressure and creatinin.

Conclusions. Several treatment options exist for RAA: resection and interposition, endovascular interventions, including covered stents and embolisation, and autotransplantation. While autotransplantation is most invasive, it allows extensive vascular reconstruction. For complex cases, interdisciplinary review is crucial to sound indication and excellent outcome.

\section{6}

\section{Postinterventionelle diagnostische und therapeutische Folgen der Stentgraftimplantation an der infrarenalen Aorta}

\author{
P. Konstantiniuk ${ }^{1}$, S. Koter ${ }^{1}$, M. Hörletzberger ${ }^{1}$, \\ J. Fruhmann ${ }^{1}$, H. Portugaller ${ }^{2}$, T. Cohnert ${ }^{1}$ \\ ${ }^{1}$ Department of Vascular Surgery, Graz, Austria; \\ ${ }^{2}$ Department of Interventional Radiology, Graz, Austria
}

Grundlagen. Seit Implementation der Aortenstentgraftimplantation wurden zahlreiche Untersuchungen zur Machbarkeit und Sicherheit der Methode durchgeführt. Der postinterventionell notwendige Aufwand wird nicht in annähernd gleicher Weise kritisch dargestellt.

Methodik. Im Zeitraum von 10/1996 bis 01/2010 wurden an der Univ. Klinik für Chirurgie in Graz 171 primäre Stentgraftimplantationen an infrarenalen Aortenaneurysmata durchgeführt. Analysiert werden Anzahl und Verteilung postinvertentioneller diagnostischer Maßnahmen (Computertomographien, diagnostische Angiographien) sowie therapeutischer Schritte (PTA, Stent, Stentgraft, offene Operation). Weiters wird die Entwicklung einer postoperativen Niereninsuffizienz näher untersucht.

Ergebnisse. 43 Patienten hatten einen oder mehrere Folgeeingriffe (29 Patienten mit 41 Interventionen, 34 Konversionen sowie 20 Patienten mit 22 anderen Operationen). Im Mittel wurden postinterventionell 9,99 Kontrastmitteluntersuchungen (Computertomographie oder 
konventionelle Angiographie) durchgeführt (Minimum 1, Maximum 35). In 6 Fällen entwickelte sich nach im Mittel 29,7 Monaten eine dialysepflichtige Niereninsuffizienz. In 12 weiteren Fällen mussten die computertomographischen Nachkontrollen wegen einer kompensierten Niereninsuffizienz abgebrochen werden. Die letzte Kontrastmittelgabe erfolgte im Mittel 40,5 Monate nach dem Primäreingriff.

Schlussfolgerungen. In allfälligen Kostenvergleichen zwischen konventioneller und interventioneller Sanierung eines infrarenalen Aortenaneurysmas müssen auch der postoperative bzw. postinterventionelle Aufwand miteinbezogen werden.

\section{7}

Das Auftreten von Endoleaks nach endovaskulärer Rekonstruktion eines infrarenalen Aortenaneurysmas in Abhängigkeit vom Prothesentyp

\section{P. Konstantiniuk ${ }^{1}$, S. Koter ${ }^{1}$, M. Hörletzberger ${ }^{1}$, S. Schweiger ${ }^{1}$, H. Portugaller ${ }^{2}$, T. Cohnert $^{1}$ \\ ${ }^{1}$ Department of Vascular Surgery, Graz, Austria; ${ }^{2}$ Department of Radiology, Graz, Austria}

Grundlagen. Endoleaks nach Aortenstentgraft sind eine gut bekannte und untersuchte Komplikation. Ziel dieser Untersuchung ist die Evaluation der Abhängigkeit vom Prothesentyp.

Methodik. Im Zeitraum von 10/1996 bis 01/2010 wurden an der Univ. Klinik für Chirurgie in Graz 171 primäre Stentgraftimplantationen an infrarenalen Aortenaneurysmata durchgeführt. Analysiert werden endoleakfreies Überleben nach Kaplan Meier sowie dessen Abhängigkeit vom Prothesentyp durch eine Cox-Regression.

Ergebnisse. 29 Patienten entwickelten ein Endoleak I, wobei die Excluder ${ }^{\circledR}$-Prothese besonders wenig Leaks zeigte (Trend paarweise bis $p=0,07$ ). 35 Patienten zeigten ein Endoleak II, wobei die Excluder ${ }^{\mathbb{B}}$-Prothese deutlich mehr Leaks hatte (paarweise bis $p=0,05$ ). Endoleaks III wurden bei 12 Patienten gesehen. Diese traten überwiegend bei der Vanguard ${ }^{\mathbb{R}}$-Prothese auf (overall $p=0,01$ ). Ein Endoleak IV wurde bei 2 Patienten mit einer Talent ${ }^{\mathbb{R}}$-Prothese gesehen (wegen der geringen Eventzahl keine Statistik).

Schlussfolgerungen. Die vorhandenen Daten zeigen die Stärken und Schwächen der einzelnen Prothesentypen. Diese Analyse könnte für die gezielte Weiterentwicklung verwendet werden.

\section{8}

Der Stellenwert der ultraschall-geleiteten Schaumsklerosierung (SSKL) in der Behandlung insuffizienter transfaszialer venöser Kommunikationen

\section{J. Dlask, H. M. Vischer}

Landesklinikum Waldviertel Gmünd, Chirurg. Abteilung, Gmünd, Austria

Grundlagen. Die nachhaltige Unterbrechung venöser Refluxe ist die Grundlage der Behandlung der primären Varikose. Die dazu erforderlichen Eingriffe, invasiv (Op modifiziert nach Babkock) und „semi-invasiv“ (ThermoAblation), werden hierzulande vorwiegend im Rahmen von KH-Aufenthalten vorgenommen.

Methodik. Die SSKL stellt ein alternatives Verfahren dar, dass die Unterbrechung venöser Refluxe ermöglicht, im extra-muralen Bereich kostengünstig angewandt werden kann und somit eine effiziente und komfortable Alternative zum hierzulande etablierten Behandlungspfad darstellen könnte.

Ergebnisse. Von Jänner 2010 bis Jänner 2011 wurden 183 insuffiziente transfasziale venöse Kommunikationen (113 Patienten, 144 Beine) extramural mit SSKL behandelt. 157 wurden nachuntersucht. In 81,6\% $(n=128)$ konnte ein vollständige Verschluss erreicht werden und in $10,8 \%(n=17)$ die Behebung des venösen Reflux bei unvollständigem Verschluss, aber subjektiver Beschwerdefreiheit. In $7.6 \%(n=12)$ erwies sich die SSKL als ineffektiv. Wurden im Zeitraum Jänner 2009 bis Jänner 2010 noch $100 \%$ der Patienten wegen nachgewiesener insuffizienter transfaszialer venöser Kommunikationen zur stationären Behandlung zugewiesen, waren es von Jänner 2010 bis Jänner 2011 nur noch $29 \%$.

Schlussfolgerungen. Die SSKL stellt aus unserer Erfahrung eine patientenkomfortable, kostengünstige Alternative in der Behandlung der primären Varicose dar. Dargestellt werden Kosten, Komplikationen, Krankenstandsdauer, Patientenzufriedenheit sowie der Stellenwert der SSKL im Rahmen der Behandlung des venösen Ulcus Cruris und des Crosse-Rezidives.

\section{9}

\section{Auswirkungen der endovenösen Laserobliteration mit dem $1470 \mathrm{~nm}$ Laser auf das Lymphsystem}

\author{
M. Schreiner ${ }^{1,2}$, E. Huber ${ }^{1,2}$, A. Shamiyeh ${ }^{1,2}$ \\ ${ }^{1}$ Ludwig Boltzmann Institut für operative Laparoskopie, Linz, \\ Austria; ${ }^{2}$ AKh Linz, 2. Chirurgische Abteilung, Linz, Austria
}

Grundlagen. Die endovenöse Laserobliteration (EVL) hat sich in der Behandlung der insuffizienten Stammvene 
etabliert. Auswirkungen auf das Lymphsystem wurden bisher nicht untersucht.

Methodik. Bei 25 konsekutiven Patienten (6 Männer; 19 Frauen) wurden 36 Beine, 23 mit Stammvarikositas der VSM und 13 mit Stammvarikositas der VSP mittels EVL behandelt. Bei allen wurde der Beinumfang der behandelten Seite(n) an standartisierten Punkten präoperativ, 10 Tage und 6 Wochen postoperativ gemessen. Ermittelt wurde die Umfangdifferenz vor und nach der OP. Mittels Sonographie wurde Anzahl und Umfang der sichtbaren Lymphnknoten in der Leiste (VSM) oder Kniekehle (VSP) gemessen, fotodokumentiert und der präoperative Befund nach 10 Tage und 6 Wochen postoperativ verglichen.

Ergebnisse. Die VSM Gruppe zeigte postoperativ eine Zunahme des medianen Lymphknotendurchmesser in der Leiste der behandelten Extremität. Bei der VSP Gruppe konnte keine eindeutigen Veränderungen hinsichtlich des Lk-Durchmessers postoperativ festgestellt werden. Beim Beinumfang zeigten sich im postoperativen Verlauf keine signifikanten Tendenzen.

Schlussfolgerungen. Die endovenöse Laserobliteration der insuffizienten Stammvene führt in den ersten 6 Wochen postoperativ $\mathrm{zu}$ einer geringradigen Zunahme des Lyphknotendurchmessers, ohne dabei ein pathologisches Ausmaß zu erreichen. Der Beinumfang postoperativ zeigt keine signifikanten Tendenzen. Dies lässt den Schluß zu, dass das abführende Lymphsystem keine wesentliche Beeinträchtigung durch die endovenöse Behandlung erfährt.

\section{Hauptsitzung - Berufsbild und chirurgische Karriere}

\section{5}

\section{Manuelle Intelligenz und chirurgische Ausbildung}

\section{G. Lexer, K. H. Simonitsch, D. Hierm, B. Liebhard, J. Schmerlaib}

Abteilung für Allgemeinchirurgie, LKH Wolfsberg, Wolfsberg, Austria

Prof. F. R. Wilson, Neurologe, Universität San Francisco, vertritt in seinen Arbeiten „Die Hand - Geniestreich der Evolution“ die faszinierende These „Hände denken“.

Das zunehmend extrem erweiterte Bewegungsrepertoire der Hand mündet in kongnitiven Möglichkeiten des Gehirns, führt in der Vernetzung von Hand und Gehirn zur Neuordnung von Schaltkreisen. Die dynamische Verflechtung von Hand, Denken, Sprache und letztlich auch der feinmotorisch ausgeführten Schrift drückt sich im Begriff manuelle Intelligenz aus.
Das hat uns an der chirurgischen Abteilung des LKH Wolfsberg veranlaßt, für die in Ausbildung stehenden Chirurgen einen Workshop mit einem renommierten Künstler zum Thema „Manuelle Intelligenz“ durchzuführen.

Die Aufgabenstellung im Kurs forciert die Selbständigkeit. Der Teilnehmer muß, auf sich selbst gestellt, nach entsprechender Erklärung und Zielangabe, Motiv/Thema bewältigen.

Er befindet sich in einer Real- und Stresssituation und strebt danach, ein entsprechendes Ergebnis zu verbildlichen. Besonderes Augenmerk gilt der schwierigen AugeHand-Zeichnung.

Eine Woche lang, mindestens 6 Stunden pro Tag, wurden anatomische Zeichnungen und Akte, zum Teil zeitlich begrenzt, um diese Real- und Streßsituationen zu simulieren, durchgeführt.

Die Aufgabenstellung ermöglicht kein Unterbrechen, ein halbfertiges Bild wird unmittelbar als inakzeptabel empfunden. Die Anschaulichkeit des Endproduktes hat Priorität.

Das Ergebnis war eine Motivationssteigerung und eine Verbesserung der anatomischen Betrachtungsweise.

\section{Arbeitsgemeinschaft für Coloproctologie}

\section{6}

Ultratiefe Anastomose oder

Descendostoma? - Lebensqualität im Langzeitverlauf bei tiefsitzendem Rektumkarzinom erhoben mittels EORTC QLQ-C30 und QLQ-C38

\section{R. Kafka ${ }^{1}$, C. Zambanini ${ }^{1}$, B. Holzner ${ }^{2}$, J. Giesinger ${ }^{3}$, J. Pratschke ${ }^{1}$, M. Zitt ${ }^{1}$ \\ ${ }^{1}$ Universitätsklinik für Viszeral-, Transplantations- und Thoraxchirurgie, Innsbruck, Austria; ${ }^{2}$ Universitätsklinik für Psychiatrie, Psychoonkologische Ambulanz, Innsbruck, Austria; ${ }^{3}$ Universitätsklinik für Psychiatrie, Klin. Abt. f. Biolog. Psychiatrie, Innsbruck, Austria}

Grundlagen. Beim tiefsitzendem Rektumkarzinom konnte in den letzten Jahren aufgrund technischer Fortschritte die Exstirpationsrate deutlich gesenkt werden. Ultratiefe Anastomosen sind häufig mit partieller Inkontinenz und Entleerungsstörungen vergesellschaftet, während eine definitive Deszendostomie ebenfalls Einschränkungen in puncto Lebensqualität bedeutet.

Ziel dieser retrospektiven Untersuchung ist es mittels eines standardisierten Fragenbogens der EORTC (QLQ- 
C30 und QLQ-C38) Unterschiede in der Lebensqualität im Langzeitverlauf zwischen Patienten mit ultratiefer Anastomose (Tumorunterrand $\leq 4 \mathrm{~cm}$ ) oder Descendostomie und Unterschieden zwischen Männern und Frauen zu erheben.

Ergebnisse. Zum Befragungszeitpunkt tumorfrei und mit der Befragung einverstanden waren 22 Patienten mit ultratiefer Anastomose und 24 Patienten mit Descendostoma, im Alter von 69 (36-90) median (range); davon 15 Frauen und 24 mit neoadjuvanter RCTX.

Schlussfolgerungen. Während sich die im Vergleich zur Normalbevölkerung relative hohe globale Lebensqualität nicht unterscheidet, zeigen sich im Bereich der physischen Funktionsfähigkeit mit 82 zu 62 und Schmerzen Vorteile der ultratiefen Anastomose, im Bereich Duchfall

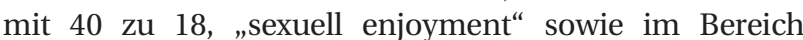
Körperbild bei Frauen Vorteile für das Desendostoma. Unterschiede in einzelnen Bereichen der Lebensqualität vor allem im Bereich Sexualität und body image sollten genauer untersucht werden, um eine individuelle Therapieentscheidung treffen zu können.

\section{7}

\section{Permanent stoma after sphincer- preserving resection for rectal cancer}

\section{A. J. Dinnewitzer, T. Fitzka, D. Öfner-Velano}

Universitätsklinik für Chirurgie der PMU Salzburg, Salzburg, Austria

Background. Resections for rectal cancer aim to remove the tumor completely with preservation of the anal sphincter, so a permanent stoma can be avoided. The purpose of this study was to assess the rate of patients with a permanent stoma after sphincter-preserving resection for rectal cancer.

Methods. 194 out of 244 consecutive patients with primary rectal cancer between 2003 and 2009 were treated with sphincter-preserving resection. Five (3\%) patients who died during the postoperative course were excluded from the analysis. All data were collected prospectively. The median age was 65 years; $40 \%$ were women, $42 \%$ underwent neoadjuvant therapy. Median follow up was 30 months.

Results. During the study period $11(6 \%)$ out of 189 patients ended up with a permanent stoma. Seven patients required an end colostomy, two patients a loop colostomy and another two patients a loop ileostomy. In six patients the reason for a permanent stoma was anastomotic failure. In four patients the reason was fecal incontinence, one patient received a permanent stoma due to progressive tumor disease.

Conclusions. Six percent of patients operated for primary rectal cancer with anastomosis ended up with a permanent stoma. So this has to be taken into account during patient's information.

\section{8}

Sinnhaftigkeit des protektiven lleostomas in der Chirurgie des Rektumkarzinoms Nutzen oder Schaden für Lebensqualität und Outcome?

\section{Zitt, I. Kronberger, P. Kogler, S. Scheidl, P. Gehwolf, F. Cakar-Beck, J. Pratschke, R. Kafka-Ritsch}

Univ.-Klinik für Visceral-, Transplantations- und Thoraxchirurgie, Medizinische Universität Innsbruck, Innsbruck, Austria

Grundlagen. Das protektive Ileostoma soll dazu dienen, kolo-rektale/kolo-anale Anastomosen nach Rektumresektion zu schützen, und somit Anastomoseninsuffizienzen zu verhindern oder zumindest deren Folgen zu minimieren. Während sich die Totale Mesorektale Exzision (TME) als Standardtechnik beim Rektumkarzinom durchgesetzt hat, herrscht in Bezug auf die Sinnhaftigkeit eines protektiven Ileostomas nach wie vor eine kontroverse Diskussion.

Methodik. Seit etwa 10 Jahren wird an unserer Abteilung bei einer tiefen Rektumresektion mit TME routinemäßig ein Ileostoma angelegt. Das eigene Patientengut und die rezente Literatur werden in Bezug auf Komplikationen, Lebensqualität und onkologischem Outcome aufgearbeitet. Dabei wird auch der Ileostoma-Verschluß in die Analyse inkludiert.

Ergebnisse. In Bezug auf die Rate an Anastomoseninsuffizienzen/Reoperationen zeigten sich in Metaanalysen basierend auf randomisierten Studien signifikante Vorteile für das protektive Ileostoma. Andere prospektive Beobachtungsstudien erbrachten auch einen signifikanten Vorteil bezüglich Mortalität. Im Gegensatz dazu ist anzumerken, dass sowohl die Ileostoma-Anlage als auch der Verschluß häufig mit Komplikationen behaftet sind. Die Datenanalyse des eigenen Patientengutes wird am Hintergrund der rezenten Literatur präsentiert.

Schlussfolgerungen. Nach derzeitiger Datenlage ist bei einer tiefen Rektumresektion mit TME die Anlage eines Ileostomas zu empfehlen. Ob alle Patienten davon profitieren oder ob das protektive Ileostoma Patienten mit spezifischen Risikofaktoren vorenthalten sein sollte, müssen weitere Studien beantworten.

\section{9}

Die protektive doppelläufige lleostomie mit prevadh ${ }^{\circledR}$ Antiadhäsionsfilm zum frühzeitigen Stomaverschluss

\section{G. Köhler, W. Zaglmair, F. Kurz, M. Aufschnaiter}

BHS Linz, Chirurgie, Colorectales Zentrum, Linz, Austria 
Die Indikation zur Anlage eines protektiven Stomas besteht mit Empfehlungsgrad 1A und Evidenzlevel 1b3b für die tiefe anteriore Rectumresektion und die Proctokolektomie mit ileoanaler Pouchanastomose. Auch bei unsicheren Anastomosen, Insuffizienzen oder entzündlichen Erkrankungen bzw. Perforationen im unteren GIT mit Peritonitis wird oftmals eine protektive Stomierung durchgeführt. Die Rückverlagerung des protektiven Stomas wurde bis dato aufgrund der zuvor sehr vulnerablen Gewebeschichten frühestens nach 10-12 Wochen empfohlen.

In der Literatur existiert kein evidenter Vorteil zugunsten der Transverso oder Ileostomie. Das Transversostoma wird an unserer Abteilung als Deviationsstoma bei Patienten mit tiefen anterioren Rectumresektionen bevorzugt angelegt. Diese Stomata eignen sich aus heutiger Sicht vor allem aufgrund der lokalen Schwellneigung nicht für den frühzeitigen Verschluss. Durch die Applikation eines prevadh ${ }^{\circledR}$ Antiadhäsionsfilms im Rahmen der Ileostomieanlage, kann die Stomarückverlagerung nach Anastomosenabheilung, also am 10-14 Tag im Rahmen des stationären Aufenthaltes einzeitig erfolgen. Obligat ist eine Anastomosendichtigkeitsprüfung mit Kontrasteinlauf und eine endoskopische Inspektion. Es unterbleiben das oft sehr mühevolle Anlernen der Stomaversorgung und die psychosoziale Beeinträchtigung.Desweiteren verringern sich stomaassozierte Komplikationsmöglichkeiten wie Prolaps, Stenose, Retraktion, Nekrose, Blutungen, high output, peristomale Hautschäden und parastomale Hernienbildungen. Auch kann aus einer längerfristigen Stomierung eine Sphincterschwäche mit schlechteren Bedingungen zum Stomaverschluss resultieren. Wir möchten diese Methode in der klinischen Anwendung vorstellen und über unsere ersten Erfahrungen berichten.

\section{0}

\section{Innsbrucker Erfahrungen bei der operativen Behandlung des Rektumprolapses von 2003-2010}

\section{T. Resch, M. Oberwalder, J. Pratschke, F. Aigner}

Universitätsklinik für Visceral-, Transplantations- und Thoraxchirurgie Innsbruck, Innsbruck, Austria

Grundlagen. Das Ziel dieser retrospektiven Studie ist es, die Indikationen und die Ergebnisse der Operationen bei III ${ }^{\circ}$ Rektumprolaps in einem Zentrum zu untersuchen.

Methodik. Alle zwischen April 2003 und Dezember 2010 wegen III $^{\circ}$ Rektumprolaps behandelten Patienten wurden retrospektiv analysiert. Nachuntersuchungen wurden anhand klinischer Kontrollen in definierten Zeitabständen und telefonischer Umfragen durchgeführt.

Ergebnisse. 36 Patienten (33 weiblich; Ø 63,2; min. 29, max. 87 Jahre) wurden wegen $\mathrm{III}^{\circ}$ Rektumprolaps an unserer Abteilung behandelt. Zwölf Patienten wurden über einen perinealen Zugang (OP nach Rehn-Delorme 3; OP nach Altemeier $=9,12$ weiblich) und 24 Patienten über einen abdominellen Zugang operiert (konventionelle Rektopexie $=1$, konventionelle Resektionsrektopexie $=6$; laparoskopische Rektopexie =11; laparoskopische Resektionsrektopexie $=6 ; 21$ weiblich). Hauptindikation für das perineale Vorgehen war das stark erhöhte Operationsrisiko bei alten Patienten. Das mittlere Follow-up betrug 47,8 Monate (min. 1,4 max. 94 Monate). Die Gesamtrezidivrate betrug über den Beobachtungszeitraum 19,4\% $(n=7)$, wobei 3 Rezidive bei den perinealen Eingriffen $\quad($ Rehn-Delorme $=1, \quad$ Altemeier $=2)$ und 4 Rezidive bei den abdominellen Eingriffen (konventionelle Resektionsrektopexie $=2$, laparoskopische Rektopexie $=1$, laparoskopische Resektionsrektopexie $=1$ ) auftraten. Die präoperative $\mathrm{III}^{\circ}$ Stuhlinkontinenz bei 11 Patienten $(30,5 \%)$ verbesserte sich postoperativ deutlich (1 Patient, $2,7 \%)$.

Schlussfolgerungen. Unsere retrospektive Analyse zeigte einen Vorteil für die laparoskopischen Eingriffe in Bezug auf die Rezidivrate. Die Stuhlinkontinenzrate konnte im Allgemeinen deutlich verbessert werden.

\section{1}

Perineal stapled prolaps resection (PSP) for external rectal prolapse: an elegant, fast and safe procedure

\section{B. Muggli, H. E. Wagner}

Department of Surgery, Spital STS AG, Thun, Switzerland

A new surgical technique, the Perineal Stapled Prolaps resection (PSP) for external rectal prolapse was introduced in a feasibility study in 2008. This analysis reports our earliest results in the new procedure.

From May 2010 to December 2010 Perineal Stapled Prolaps resection was performed on patients with external rectal prolapse. The prolapse was completely pulled out and then axially cut open with a linear stapler at three and nine o'clock in lithotomy position. Finally, the prolapse was resected stepwise with the curved Contour Transtar stapler at the prolapse's uptake. There were no intra- or early postoperative complications and there is no documented recurrence. Median operation time was 26 minutes (20-35), hospital stay 5 days (1-9).

The Perineal Stapled Prolaps resection (PSP) is an elegant, fast and safe procedure with good functional results. There were no minor or major complications and there is no documented recurrence. 


\section{2}

Die perineale Staplerresektion

in der Behandlung des Rektum- und Stomaprolaps - ein Erfahrungsbericht

\section{Binder, R. Klug}

Chirurgie, LK Horn, Horn, Austria

Beim Rektumprolaps handelt es sich um eine Erkrankung des höheren Lebensalters. Es sind vorwiegend Frauen betroffen. Der Leidensdruck ist mitunter erheblich. Neben den klassischen offenen bzw. laparoskopischen abdominellen Verfahren kommen bei älteren Patienten die weniger belastenden peinealen Verfahren zur Awendung. Diese haben allerdings einerseits den Nachteil einer hohen Rezidivrate, andererseits sind sie chirurgisch aufwendig. Wir berichten über unsere Erfahrung mit der perinealen Staplerresektion als zügige, wenig belastende Methode in der Therapie des Rektumprolaps und ihre erweiterte Anwendungsmöglichkeit beim Stomaprolaps.

\section{3}

\section{Internal rectal prolapse - STARR} procedure - functional results after 10 years

\section{Glöckler}

Department of Surgery, St. Elisabeth Hospital, Vienna, Austria

Background. The STARR-procedure (Stapled-Transanal-Rectal-Resection) removes an internal rectal prolapse. The indication is given by the symptomload, mainly obstructed defecation syndrome (ODS). The purpose of this single group prospective trial was to evaluate the long-term functional results of the STARR-procedure.

Methods. 203 selected female patients (mean age: 55.4, range: 20-82), treated by STARR between 1999 and 2002, were enrolled into a continuous follow-up. A symptom score - ODS score 0-40 (0: no obstruction, 40: severe obstruction) - was rated and compared preoperative, at 6 months and at the last follow-up. Patients preoperatively had to score in the range 7-40 (mean: 18.7, sd: 6.2).

Results. After 6 months the mean ODS score was 1.2 (61.6\% of the patients scored zero and $94.1 \%$ in the range $0-6$ ). After a mean follow-up of 7.8 years (sd: 2.5) a mean score of 2.3 was rated $(64.5 \%$ scored zero and $86.2 \%$ in the range $0-6) .84 .7 \%$ of the patients judged their results as "excellent" or "good" at the last follow-up.

Conclusions. The significant and stable reduction of the ODS score after the STARR-procedure underlines its benefit for selected patients. The score related successrate is more than $85 \%$ after a mean follow-up of 7.8 years.
Österr. Ges. für Orthopädie

und Österr. Ges. für Plastische, Ästhetische und Rekonstruktive Chirurgie: Weichteiltumore

\section{6}

\section{Operative Behandlung von Tumoren peripherer Nerven}

\section{W. Girsch}

Orthopädisches Spital Speising, Wien, Austria

Grundlagen. Tumore peripherer Nerven, Neurofibrome, Neurinome und Schwannome sind selten und $\mathrm{zu}$ überwiegend gutartig. Symptome sind zunächst meist Schmerzen, später ein schleichender Funktionsverlust an den Endorganen. Die Diagnostik, Elektroneurographie, Ultraschall und MRT erlaubt üblicherweise keine Differenzierung der Dignität der benignen von den malignen Varianten, Fibrosarkom und malignes Schwannom.

Methodik. Tumoröse Veränderungen am peripheren Nerven erfassen entweder das gesamte Bindegewebe (Neurofibrome) oder einzelne Faszikel (Neurinome, Schwannome). Entsprechend darf eine PE nicht keilförmig aus dem veränderten Nervenanteil erfolgen. Der Nerv muss intraneural disseziert, dh in seine Faszikel aufgetrennt werden um das veränderte Nervengewebe unter gleichzeitiger Schonung der nicht veränderten Anteile resezieren zu können, was meist einer marginalen Tumorresektion entspricht.

Ergebnisse. Die PE mit marginaler Resektion von Nerventumoren erlaubt meist einen weitgehenden Funktionserhalt der betroffenen Nerven und stellt für die benignen Nerventumore bereits die definitive Behandlung dar. Erst die gesicherte Diagnose eines malignen Nerventumors ergibt die Indikation zur radikalen Resektion und der damit erforderlichen Rekonstruktion.

Schlussfolgerungen. Die operative Behandlung peripherer Nerventumore unterscheidet sich deutlich von der Behandlung sonstiger Weichteiltumore und sollte nur von mikrochirurgisch Kundigen unter Einsatz von Sehbehelfen, Lupenbrille oder Mikroskop erfolgen.

\section{7}

\section{Kindliche Tumore an der Hand}

\section{Karner ${ }^{1}$, G. Weigel ${ }^{2}$, W. Girsch ${ }^{1}$}

${ }^{1}$ Orthopädisches Spital Speising, Abt. f. Kinder- und Jugendorthopädie, Wien, Austria; ${ }^{2}$ Medizinische Universität Graz, Universitätsklinik für Chirurgie, Klin. Abt. f. Plastische und Rekonstruktive Chirurgie, Graz, Austria 
Grundlagen. In der Kinderambulanz des Orthopädischen Spitals Speising wurden 2001 und 2002 bei weit mehr als 500 Neuvorstellungen 18 Kinder mit der Überweisungsdiagnose Tumor oder Tumorverdacht an der Hand vorstellig.

Methodik. Das Patientengut umfasste 18 Kinder (3 Buben, 15 Mädchen) in einem mittleren Alter von 9 Jahren (3-16a). Bei 14 Patienten waren die Weichteile, in 4 Fällen war das Skelett betroffen.

Ergebnisse. Die Diagnostik erbrachte folgende Diagnosen: $4 \times$ Ganglion, $5 \times$ Synovialitis, $4 \times$ Fibromatoseverdacht, $4 \times$ Veränderungen im Knochen, $1 \times$ Tumorverdacht (als posttraumatisch gewertet und konservativ ausbehandelt).

Die Diagnose Ganglion wurde histologisch bestätigt. Zwei Synovektomien verifizierten die Verdachtsdiagnose: rheumatoide Arthritis. Eine Synovitis der Beugesehnen erwies sich als Neurofibrom des N. medianus. Die histologische Diagnostik der fibromatoseverdächtigen Tumoren ergab: reaktives Weichteilgewebe, kalzifizierendes aponeurotisches Fibrom, Fibromatose, Lymphangiom. Die Untersuchung der Knochentumore ergab histologisch: $2 \times$ Enchondrom, $1 \times$ fibrös ossärer Pseudotumor, $1 \times$ fibröse Dysplasie.

Schlussfolgerungen. Tumore der Hand sind bei Kindern selten, betreffen üblicherweise die Weichteile und das Skelett und stellen in der Mehrzahl der Fälle benigne Läsionen dar. Die Diagnostik sollte eine exakte Anamnese, eine klinische Untersuchung, Röntgen-Standardaufnahmen, Ultraschalluntersuchungen (Weichteiltumore), MRT-Untersuchung in ausgewählten Fällen umfassen. Eine operative Intervention ist, abgesehen von Knochenzysten, bei jeder tumorösen Veränderung zumindest aus Gründen der Diagnosesicherung indiziert.

\section{8}

The impact of re-excision and residual disease of inadequately resected soft tissue sarcomas on surgical therapy, results and prognosis - a single institution experience with 682 patients

\section{P. T. Funovics, S. Vaselic, J. Panotopoulos, V. Stockhammer, M. Dominkus, R. Windhager}

Department of Orthopaedic Surgery, Vienna General Hospital, Medical University of Vienna, Vienna, Austria

Background. Due to their rare incidence soft tissue sarcomas (STS) are often resected without clear margins. The aim of this study was to investigate the impact of reexcision of STS on survival.

Methods. Out of 752 patients with STS (406 men and 346 women, average age 51 years), 310 patients were referred after an inadequate resection, 442 patients presented for primary treatment. 682 patients were compared over a mean period of 65 months (median,
36 months) according to the treatment groups regarding their survival, clinical, surgical and pathological data.

Results. The five-year survival rate of 621 surgically treated patients was $53.9 \%$. There was a continuous improvement in survival during the whole treatment period in the respective decades. The overall survival rate in both groups was not significantly different regardless of low- or high-grade malignancies. Patients with a re-resection did not have a higher rate of local recurrences; patients with a primary resection had a worse prognosis regarding metastases. A re-resection within 12 weeks indicated a better prognosis.

Conclusions. An inadequate excision of a STS does not cogently mean deterioration of overall survival, but necessitates an ample and quick re-resection.

\section{9}

\section{Desmoidtumor \& Brustimplantat: Fallbericht und Literaturübersicht}

\section{R. Pauzenberger, G. Djedovic, T. Bauer, G. Pierer, A. Schwabegger}

Universitätsklinik für Plastische-, Rekonstruktive- und Ästhetische Chirurgie Innsbruck, Innsbruck, Austria

Grundlagen. Desmoidtumore stellen mit zirka $0.2 \%$ eine Rarität unter den Mammatumoren dar. Dieser Bindegewebstumor wächst lokal infiltrativ und destruierend, jedoch ohne die Fähigkeit zu metastasieren, und weist eine hohe Rezidivrate auf. Der Desmoidtumor stellt eine diagnostische Herausforderung dar, da er klinisch und radiologisch als Mammakarzinom mißinterpretiert werden kann.

Die Ätiologie ist unklar und ein kausaler Zusammenhang zwischen Brustimplantaten und dem Auftreten von Desmoidtumoren kann aktuell nicht bestätigt werden, ist jedoch aufgrund der Seltenheit auch schwer nachzuweisen. In der Literatur sind derzeit 23 Fälle von Implantatassoziierten Desmoidtumoren publiziert

Methodik. Wir berichten über eine 50-jährige Patientin, bei der drei Jahre nach Mammaaugmentation durch Silikonimplantate eine Läsion im Bereich der rechten Brust festgestellt wurde. Die MRT zeigte eine höchst suspekte inhomogene Raumforderung. Die weitere stanzbioptische Abklärung ergab die Diagnose Desmoidtumor. Eine weite Tumorresektion mit partieller Thoraxwandresktion und Rekonstruktion konnte komplikationslos durchgeführt werden.

Ergebnisse. Sechs Monate nach R0-Resektion ist die Patientin rezidivfrei und mit dem funktionellen und ästhetischen Ergebnis zufrieden.

Schlussfolgerungen. Wissenschaftliche Evidenz, Mammaimplantate als Risikofaktor für die Entstehung von Desmoidtumoren der Brust zu sehen, ist aktuell auf Grund der bekannt geringen Fallzahlen nicht gegeben. Die Therapie der Wahl ist die Resektion im Gesunden. Regelmäßige Tumornachsorge ist wegen der hohen Rezidivrate indiziert. 


\section{0}

\section{Präoperative Nervendarstellung bei Tumorresektionen mittels Diffusions Tensor Traktographie am Beispiel von peripheren Nerventumoren}

\section{Schmidt ${ }^{1}$, G. Kasprian ${ }^{2}$, I. Pona ${ }^{1}$, A. Hold ${ }^{1}$, O. Aszmann ${ }^{1}$, M. Frey ${ }^{1}$}

${ }^{1}$ Abteilung für Plastische und Rekonstruktive Chirurgie, MUW, Wien, Austria; ${ }^{2}$ Univ. Klink für Radiologie, MUW, Wien, Austria

Grundlagen. Diffusions Tensor Traktographie (DTT) ist eine kürzlich entwickelte nicht invasive MR-Technik. Das Ziel dieser Studie war die Wertigkeit der DTT in der Therapie von peripheren Nerventumoren zu untersuchen. Insbesondere die Möglichkeit zur präoperativen Visualisierung intakter Nervenfaszikel mit korrektem anatomischen Faszikelverlauf und Differenzierung dieser Faszikel von Tumorgewebe sollte überprüft werden.

Methodik. Patienten mit dem klinischen Verdacht auf einen peripheren Nerventumor wurden im Rahmen einer prospektiven Studie mittels DTT-MRT untersucht. Der Verlauf und die Position intakter Nervenfaszikel in Relation zum Tumor wurden intraoperativ exakt fotodokumentiert. Diese klinischen Befunde wurden anschließend mit den präoperativen DTT- Bildern verglichen und der Grad an Übereinstimmung bestimmt.

Ergebnisse. Bei 12 Patienten (durchschnittliches Alter 42 Jahre) mit peripheren Nerventumoren wurde eine DTT-MRT durchgeführt. Bei 10 Patienten wurde der Tumor reseziert. Bei acht dieser zehn Patienten zeigte sich eine hohe Korrelation der mittels DTT dargestellten intakten Nervenfaszikeln und der intraoperativen Anatomie.

Schlussfolgerungen. Mittels DTT konnte eine korrekte präoperative Visualisierung intakter Nervenfaszikel mit anatomischer Faszikelposition und Differenzierung dieser Faszikel von Tumorgewebe durchgeführt werden. Diese Information zeigte sich als wertvoll bei der Planung der Tumorresektion und eventuell notwendigen Nervenrekonstruktion. Limitationen der Methode offenbarten sich bei extensiven komplexen Plexopathien, bei Nahebeziehungen zu großen Gefäßen und bei Tumordurchmesser kleiner als $5 \mathrm{~mm}$.

\section{1}

Die Vorteile des anterolateralen

Oberschenkellappens bei der Weichteilrekonstruktion in der KopfHals-Region

F. Ensat ${ }^{1}$, H. Schubert ${ }^{1}$, M. Hladik ${ }^{1}$, E. Russe ${ }^{1}$, J. Beck ${ }^{2}$, G. Rasp ${ }^{3}$, G. Wechselberger ${ }^{1}$

${ }^{1}$ Abteilung für Plastische- und Wiederherstellungschirurgie, Krankenhaus der Barmherzigen Brüder, Salzburg, Austria;
${ }^{2}$ Univ. Klinik für Mund- Kiefer-und Gesichtschirurgie, Landeskrankenhaus Salzburg, Salzburg, Austria; ${ }^{3}$ Univ. Klinik für Hals- Nasen-und Ohrenkrankheiten, Landeskrankenhaus Salzburg, Salzburg, Austria

Grundlagen. Kaum eine Region stellt so viele unterschiedliche Ansprüche bei der Defektdeckung wie der Kopf- Halsbereich. Auf kleinsten Raum sind Hautund Weichteildefekte, oft in Kombination Schleimhautdefekten zu decken. Wir beschreiben in dieser Arbeit unsere Erfahrungen nach der Verwendung von insgesamt 23 ALT Lappenplastiken zur Defektdeckung im Kopf- Halsbereich während der vergangenen 18 Monate.

Methodik. Es erfolgte die retrospektive Analyse des Krankheitsverlaufs von 22 Patienten welche zwischen Dezember 2008 und Juni 2010 aufgrund eines Weichteildefekts im Kopf- Halsbereich mit einer ALT Lappenplastik versorgt wurden. Neben Variationen des Hebevorganges wurden Indikationen, Lappencharakteristika, Komplikationen, Nebendiagnosen und klinische Ergebnisse evaluiert.

Ergebnisse. Die Länge des Pedikels betrug im Durchschnitt 9,5 cm,die Größe der Hautinsel $5 \times 12 \mathrm{~cm}$ (4-7 mal 5-16 cm). Der Perforator verlief in 10 Fällen intramuskulär, in 13 Fällen septokutan. Bei 2 Patienten musste aufgrund von Durchblutungsproblemen revidiert werden, alle übrigen Lappenplastiken zeigten minimale oder keine Auffälligkeiten im postoperativen Verlauf. Alle Hebestellen konnten direkt verschlossen werden, lediglich in 2 Fällen kam es zu Wundheilungsstörungen an der Entnahmestelle welche konservativ beherrscht werden konnten.

Schlussfolgerungen. Der lange und kaliberstarke Gefäßstiel und verschiedenen Möglichkeiten der Gewebskomposition bei minimaler Hebedefektmorbidität machen den ALT zu einem hervorragend geeigneten Lappen für die Weichteilrekonstruktion im Kopf- Hals Bereich.

\section{Hauptsitzung -}

Komplikationsmanagement 5: Dehiszenz der ösophagogastrischen oder ösophagojejunalen Anastomose, Ösophagusperforation

\section{3}

\section{Zum Stellenwert von Stentungen in der Chirurgie des thorakalen Oesophagus}

\section{Möschel, M. Hufschmidt, K. Ammann, P. Tschann, E. Wenzl}

Department of General and Thoracic Surgery, Feldkirch, Austria 
Grundlagen. Stentungen, 1924 durch Sir HENRY SOUTTAR inauguriert, werden in Bezug auf Effizienz und „outcome“ im primären therapeutischen Ansatz und im Management der bekannt hohen Komplikationsbelastung der thorakalen Oesophaguschirurgie reevaluiert.

Methodik. Retrospektive „single center“-Untersuchung an 74 Patienten mit maligner, benigner oder iatrogener Pathologie mit drei Indikationsgruppen für interventionelle Prozeduren: I. Primär therapeutischer bzw. palliativer Ansatz ( $n=15)$, II. Insuffizienzen (DINDO IIIa) nach resezierenden Verfahren in VATS-, transhiataler, AKIYAMA-, IVOR-LEWIS- oder McKEOWN-Technik mit teilweise komplexer kolischer oder jejunaler Interponat-Passagerekonstruktion $(n=8)$, III. Anastomosenstenosen $(n=7)$.

Ergebnisse. Für die Indikationsgruppe III erwiesen sich interventionelle Maßnahmen in allen Fällen als zielführend, teilweise in Mehrfachsitzungen, wohingegen in der Gruppe I zusätzliche Chirurgische Maßnahmen bei 3 Patienten notwendig wurden. Die Insuffizienzen der Gruppe II entsprechen etwa $40 \%$ der postoperativen Komplikationen und wurden in $75 \%$ erfolgreich interventionel therapiert. Zusatzmaßnahmen bestanden in chirurgischen mediastinalen Abszessausräumungen $(n=3)$, einer palliativen Oesophagusresektion bei Tumorperforation sowie einer radiologisch-interventionellen Abszessdrainage. Interventionelle Prozeduren haben ein Eigenkomplikationspotential mit einer Mortalitätsrate von $15,3 \%$.

Schlussfolgerungen. Inhärentes Komplikationsrisiko und drohender fataler Verlauf bei Unzulänglichkeit oder Scheitern interventioneller Maßnahmen lassen das EARLAM'sche Statement weiterhin gültig erscheinen: „Dilatation and intubation are absolutely essential skills for the oesophageal surgeon, and should not be handed over to any other person".

\section{4}

\section{Long-term self-expanding esophageal stenting is safe and effective independent of indication}

\section{S. F. Schoppmann, F. Langer, G. Prager, J. Zacherl}

Universitätstsklinik für Chirurgie, Vienna, Austria

Background. Esophageal stenting with self-expanding plastic or covered metal stents is believed to be safe and effective. As data on the outcome of patients with longterm stenting are not available, this study was conducted to evaluate the safety and efficacy of long-term esophageal stenting.

Methods. From 2002 to 2008, 70 patients with long-term esophageal stenting for various indications from a prospective database were included into this analysis.
Results. The median stent-period 297 (124-1980) days during a mean follow-up time of $55 \pm 52.4$ months. Overall-complication rate was $15.7 \%$ with a primary successrate of $97.2 \%$. The stent-related total re-intervention rate was $11.4 \%$ and the disease-related re-intervention rate was $5.7 \%$. There were no correlations between stent-characteristics and the risk of complication or dislocation. No stent-related death was observed.

Conclusions. Our data provide evidence that long-period esophageal stenting is safe and effective for various indications.

\section{5}

\section{Ösophagusperforation - Therapie und Komplikationsmanagement}

\section{R. J. Stadlhuber, M. Schweigert, A. Dubecz, H. J. Stein \\ Klinik für Allgemein-, Viszeral und Thoraxchirurgie, Klinikum Nürnberg Nord, Nürnberg, Germany}

Grundlagen. Die spontane Ösophagusperforation infolge heftigen Erbrechens zusammen mit der traumatischen und der iatrogenen Ösophagusperforation stellt eine seltene Notfallsituation mit erheblicher Morbidität und Mortalität dar. Es treten zunehmend konservative Therapieansätze in den Vordergrund, ab 2004 haben wir die endoskopische Stent-Implantation als Primärtherapie übernommen.

Methodik. Im Zeitraum 2004-2008 wurden 14 Patienten mit einer Perforation des thorakalen Ösophagus behandelt. 8 Patienten mit einem Boerhaave-Syndrom (57\%), 4 iatrogene Perforationen ( $29 \%$ ) sowie 2 traumatische Perforationen (14\%). 11 der 14 Patienten $(79 \%)$ erhielten einen endoskopisch platzierten selbstexpandierenden Stent.

Ergebnisse. Das Durchschnittsalter betrug 65,7 Jahre. Ein Pleuraempyem entwickelten 9 Patienten (64\%), eine Mediastinitis 3 (21\%). Die Mortalität betrug 14,3\% (2/14). Bei den übrigen 12 Patienten $(85,7 \%)$ konnte ein guter Therapieerfolg kompletter Ausheilung der Perforation erzielt werden.

Für die operative Therapie wird in der Literatur eine Mortalität von ca. $20 \%$ beschrieben. Nach primärer Naht werden Nahtinsuffizienzen von $20-25 \%$ angegeben. In unserem Behandlungskonzept haben wir die endoskopische Stent-Implantation bei einer Mortalität unter $20 \%$ eingesetzt. Der Organverlust des Ösophagus konnte vermieden werden.

Schlussfolgerungen. Das Behandlungskonzept mit primärer endoskopischer Stentimplantation sowie chirurgischer Therapie septischer perforationsbedingter Komplikationen ist sicher und im hohen Prozentsatz erfolgreich. Die Ziele der primären operativen Therapie können ohne Anstieg der Mortalität ebenso erreicht werden 


\section{6}

\section{Effizienzsteigerung des präoperativen Trainings viszeralchirurgischer Anastomosentechniken}

\section{J. Gröne, J. C. Lauscher, C. Holmer, H. J. Buhr, J. Ritz}

Chirurgische Klinik I, Campus Benjamin Franklin, Charité Universitätsmedizin Berlin, Berlin, Germany

Grundlagen. Während für das Training laparoskopischer Techniken OP-Simulatoren zur Verfügung stehen, ist das Angebot für konventionelle Eingriffe begrenzt.

Ziel war die Untersuchung der Effizienz eines OPTrainers für viszeralchirurgische Anastomosentechniken in der chirurgischen Weiterbildung.

Methodik. 36 Chirurgen wurden nach ihrer operativen Erfahrung (Anfänger/Erfahrene) stratifiziert und in eine Trainingsgruppe mit („B+“; $n=17)$ und ohne Trainer („B-“; $n=19$ ) randomisiert. Zu Beginn (t1) und am Ende (t2) einer 4-tägigen Trainingsphase erfolgte die Auswertung einer Video- und Foto-dokumentierten, standardisierten fortlaufenden Darmanastomose durch 2 unabhängige Chirurgen in Bezug auf Zeit und Qualität. Um eine möglichst realistische OP-Situation zu simulieren, erfolgte die Naht zum Zeitpunkt (t2) am offenen Situs eines intubierten Hausschweines.

Ergebnisse. Erfahrene Teilnehmer waren mit und ohne Trainer signifikant schneller als die Anfänger ( $p=0,038)$. Gruppe "B+" war zum Zeitpunkt $\mathrm{t} 2$ schneller $(192,4 \pm 53.8$ vs. $221,3 \pm 47,8 \mathrm{~s} ; \quad p=0,064)$ und erzielte einen höheren Qualitäts-Score $(12,1 \pm 2,0$ vs. 10,2 $\pm 2,6$ Punkte; $p=0,032$ ) als Gruppe „B-“. Gruppe „B+“ zeigte im Vergleich zu "B-" zum Zeitpunkt t2 eine signifikante Verbesserung in der Geschwindigkeit $(p=0,049)$ und der Qualität (Darmanastomosen-Score; $p=0,039$ ).

Schlussfolgerungen. Durch den Einsatz des Trainers wird die Effizienz des präoperativen chirurgischen Trainings optimiert. Zusätzlich werden neben der eigentlichen Nahttechnik wichtige Parameter, wie die OP-Situation in Bezug auf Körperstellung zum Situs und Arbeiten im räumlich begrenzten OP-Feld realistisch vermittelt.

\section{Herzchirurgie Thoraxchirurgie: Varia}

\section{4}

Minimally invasive resection of atrial myxoma - the Innsbruck experience

\author{
A. Heinz, J. Kilo, K. Stifter, H. B. Hangler, \\ M. Grimm, L. Müller
}

Medical University of Innsbruck, Innsbruck, Austria
Background. Primary tumors of the heart are rare, with the predominant risk of embolization. Therefore, excision of myxoma should be performed soon after diagnosis. We reviewed our experience on the development of a minimally invasive surgical technique and its introduction into clinical routine.

Methods. We reviewed all patients who underwent surgical myxoma resection at our institution from $01 / 2003$ to $12 / 2010$. Analysis was performed concerning myxoma localization, patient characteristics and operative variables.

Results. From 01/2003 through 12/2010, 30 patients underwent isolated myxoma resection. In that period, 13 operations were performed in a minimally invasive technique through a right-sided minithoracotomy and remote-access perfusion through the right groin. Three myxomas were located in the right atrium, 10 in the left atrium. During the last years, the number of patients undergoing minimally invasive surgery is rising steadily (2003: $1 \mathrm{pt}, 2005$ : $1 \mathrm{pt}, 2007-08$ : 2 pts, 2009: 3 pts, 2010: 4 pts). The mean age was 62.0 years (51-83 yrs), mean weight was $77.0 \mathrm{~kg}(48-110 \mathrm{~kg})$. Mean aortic crossclamp time was $52.5 \mathrm{~min}$, mean CPB was $137.8 \mathrm{~min}$. There was no operative death and no conversion to median sternotomy. No patient presented with recurrence of myxoma.

Conclusions. Minimally invasive resection of intraatrial myxoma has successfully been introduced into clinical routine at our institution.

\section{5}

\section{Causes and consequences of postoperative atrial fibrillation (Afib) in robotic totally endoscopic coronary bypass surgery (TECAB)}

\section{F. Weidinger ${ }^{1}$, T. Schachner ${ }^{1}$, N. E. Bonaros ${ }^{1}$, B. Hofauer ${ }^{1}$, E. J. Lehr ${ }^{2}$, M. Vesely ${ }^{2}$, D. Zimrin ${ }^{2}$, J. O. Bonatti ${ }^{2}$ \\ ${ }^{1}$ Universitätsklinik für Herzchirurgie Innsbruck, Innsbruck, Austria; ${ }^{2}$ Division of Cardiac Surgery, University of Maryland Medical Center, Baltimore, USA}

Background. The aim of our study was to evaluate the incidence of AFib after TECAB and to investigate factors influencing its occurrence.

Methods. TECAB was performed in 384 patients, $73 \%$ male, aged 60 (37-90).

Results. A total of 59 patients (15.4\%) developed AFib after TECAB. Univariate analysis showed hypertension $(p=0.005)$, and increased age $(p=0.007)$, body weight $(p=0.006)$, body mass index $(p=0.005)$, EuroSCORE $(p=0.035)$, and total TECAB operation time $(p=0.01)$ to be significantly associated with AFib. We also found an increased incidence of AFib in patients undergoing hybrid interventions $(p=0.036)$ and beating heart 
TECAB $(p=0.003)$. Age $(p<0.001)$ and body weight $(p=0.003)$ were the only predictors found to be significant in multivariate analysis. Hospital mortality was $1.7 \%$ $(1 / 59)$ in the group of patients with AFib and $0.6 \%(2 / 325)$ in the group that showed no AFib after operation ( $p=$ n.s.). There was no significant 5 year survival difference in patients with and without postoperative Afib $(95.8 \% v s$. $94.5 \%, p=$ n.s.).

Conclusions. We conclude that the incidence of postoperative AFib in TECAB is relatively low. Age and body weight are the most important predictors of postoperative AFib following TECAB. Short term clinical outcome and intermediate term survival are similar in patients with and without postoperative AFib.

\section{6}

\section{Update I: usefulness of urinary biomarker NGAL in the management of renal replacement therapy (RRT, Hemofiltration)}

\section{O. Stanger ${ }^{1}$, I. Aigner ${ }^{1}$, S. Mitterwallner ${ }^{1}$, B. Bacher ${ }^{1}$, E. Haschke-Becher ${ }^{2}$}

${ }^{1}$ St. John's Hospital, Salzburg, Austria; ${ }^{2} \mathrm{KH}$ Elisabethinen, Linz, Austria

Background. Acute kidney injury (AKI) after cardiac surgery requiring initiation of hemofiltration (HF) is associated with high morbidity and mortality. Neutrophil Gelatinase Associated Lipocalin (NGAL) is a novel urinary biomarker released by renal tubules very early in response to ischemic damage. A new algorithm including NGAL was used to improve management criteria for early HF treatment.

Methods. Urinary NGAL was tested routinely in all consecutive patients (2010) before and postoperatively. HF therapy was only initiated when NGAL at 4 hours was markedly increased versus baseline and/or urine production decreased within 4 days $(<0.5 \mathrm{~mL} / \mathrm{kg} / \mathrm{h},>4 \mathrm{~h})$ independent of creatinine and urea. Safety and HF management issues were analysed.

Results. HF therapy in 2006-2009 was initiated at day 3 after surgery in $7.5 \%$ to $9.0 \%$ of patients for 7.7 days (mean). Using the new algorithm, the number of HF therapies was reduced to $4,9 \%$ (4.2 days). Preliminary data suggests no difference in extrarenal complications and permanent dialysis requirement.

.Conclusions. Urinary NGAL can be used safely as an early predictor for HF requirement in patients after cardiac surgery. Inclusion of NGAL can help in the RRT management and may reduce cost by decreasing the number of necessary HF treatments and length of therapy.

\section{7}

Der Morbus Whipple als primär kardiale Manifestation - der erste österreichische Fall einer Tropheryma-WhippeliiAortenklappenendokarditis Fallpräsentation und Literaturreview

\section{K. Mészáros ${ }^{1}$, B. Zirngast ${ }^{1}$, R. Krause ${ }^{2}$, G. Gorkiewicz ${ }^{3}$, L. Salaymeh ${ }^{1}$, H. Maechler ${ }^{1}$, K. Tscheliessnigg ${ }^{1}$ \\ ${ }^{1}$ Klinische Abteilung für Herzchirurgie, Graz, Austria; \\ ${ }^{2}$ Klinische Abteilung für Infektiologie, Graz, Austria; \\ ${ }^{3}$ Institut für Pathologie, Graz, Austria}

Grundlagen. Morbus Whippelii ist eine durch Aktinomyzeten bedingte seltene infektiöse Erkrankung, die sich meistens primär durch intestinale Symptome, enteropathische Arthritis/Spondylarthropathie, ZNS - und Augenbeteiligung manifestiert.

Eine rein kardiale Manifestation ohne begleitende extrakardiale Manifestationen ist eine Rarität.

Krankheitsverlauf: Ein 65-jähriger Patient wurde wegen eines fieberhaft-respiratorischen Infekts antibiotisch behandelt. Nach einer Woche entwickelte er einen Arteria-cerebri-anterior Infarkt mit linksseitiger Hemiparese. In der transösophagealen Echokardiographie zeigte sich eine akute Aortenklappenendokarditis mit höhergradiger AR.

Methodik. Nach Einleiten einer empirischen Antibiose mit Vancomycin, Gentamycin und Rifampicin wurde ein mechanischer Aortenklappenersatz vorgenommen. Intraoperativ zeigten sich endokarditische Vegetationen am akoronaren und rechtskoronaren Segel. Ein anamnestisch bekanntes kleines persistierendes Foramen ovale wurde verschlossen.

In den Blutkulturen konnte kein Keim nachgewiesen werden; die intraoperativ entnommenen Abstriche von der Aortenklappe und die bakteriologische Untersuchung des Operationspräparates blieb ebenso wie die Serologie auf Coxiellen, Bartonella und Mykoplasmen ohne Befund.

Ergebnisse. Erst die histopathologische Untersuchung der Aortenklappe ergab eine Infektion mit Tropheryma Whippelii. Die Antibiose wurde adaptiert auf Ceftriaxon, Doxycyclin und Hydrodychloroquine und wird als Langzeittherapie fortgeführt.

Die detaillierte Durchuntersuchung des Patienten ergab keine extrakardialen Manifestationen (intestinal, neurologisch, opthalmologisch, arthitisch) des $\mathrm{M}$. Whippelii.

Schlussfolgerungen. Drei Monate postoperativ war der Patient klinisch völlig stabil und ohne Infektzeichen; aufgrund seiner Hemiparese wurde eine ambulante neurologische Rehabilitation durchgeführt. 


\section{8}

\section{Neue Aspekte der chirurgischen Therapie der Pulmonalarterienembolie}

\section{A. E. Yates, K. Meszaros, I. Keeling, E. Beran, B. Zirngast, H. Mächler, D. Dacar, K. Tscheliessnigg}

Cardiac Surgery, Graz, Austria

Grundlagen. Die akute fulminante Pulmonalarterienembolie stellt einen lebensbedrohlichen Zustand mit akutem rechtsventrikulären Versagen und kardiogenem Schocks dar. Unsere retrospektive Studie analysiert die Resultate der chirurgischen Therapie bei Patienten mit hämodynamischer Instabilität. Die Indikation zur operativen Sanierung ist selten und nur bei der Lyse-Kontraindikation oder Lyse-Versagen bei massiven oder fulminanten Pulmonalarterienembolie zu stellen.

Methodik. Seit 1996 wurden 16 Patienten (11 Frauen, mittleres Alter 65,1 Jahre) aufgrund einer massiven oder fulminaten Pulmonalarterienembolie operiert. Fünf Patienten (31,3\%) kamen unter Reanimationsbedingungen in den Operationssaal. Drei Patienten wurden präoperativ lysiert, bei vier Patienten war diese Lyse-Therapie aufgrund von vorhergegangenen Operationen, Darmblutungen oder polytraumatischen Verletzungen kontraindiziert. Die Diagnose der PAE wurde in $50 \%$ der Fälle mittels Computerangiographie und $50 \%$ mittels Echokardiographie gestellt. Das Follow-up konnte $100 \%$ nachvollzogen werden.

Ergebnisse. Die 30-Tage-Mortalität betrug 43,8\% (7 Patienten), von denen vier präoperativ unter Reanimationsbedingungen in den Operationssaal kamen. Die restlichen neun Patienten überlebten die 30-Tage, 2 Patienten verstarben Jahre später an nicht kardialer Ursache. Die präoperativ stabilisierten Patienten zeigten bessere Operationsergebnisse als Patienten unter Reanimationsbedingungen.

Schlussfolgerungen. Die Indikation der chirurgische Embolektomie ist selten. Die Stabilisierung des Kreislaufes stellt einen wichtigen Indikator für die weitere Überlebensrate dar und kann mittels rascher Diagnostik, schneller Entscheidungsfindung und zusätzlich perkutane Herz-Kreislaufunterstützung verbessert werden.

\section{9}

Damage repair nach TEVAR: Erfahrungen mit der offen-chirurgischen thorakalen Konversion

\section{A. Greiner ${ }^{1}$, J. Grommes ${ }^{1}$, S. Langer ${ }^{1}$, R. Autschbach ${ }^{2}$, M. Jacobs ${ }^{1}$}

${ }^{1}$ Klinik für Gefäßchirurgie, Europäisches Gefäßzentrum Aachen Maastricht, Universitätsklinik der RWTH, Aachen, Germany; ${ }^{2}$ Klinik für Herz-Thorax und Gefäßchirurgie, Universitätsklinik der RWTH, Aachen, Germany
Grundlagen. Behandlungen thorakaler Aortenaneurysmen mittels Stentimplantation (TEVAR) sind nicht immer suffizient. Ist ein endovaskuläres Vorgehen dann nicht mehr möglich muss auf eine offen-chirurgische Behandlung konvertiert werden. Die Ausschaltung eines thorakalen Aneurysmas mit bereits liegenden Stentprothesen stellt eine gefäßchirurgische Herausforderung dar, welcher man sich zukünftig bei steigenden Zahlen von TEVARs stellen werden muss. Es war Ziel der Arbeit die klinischen Ergebnisse dieser Konversionsoperationen $\mathrm{zu}$ analysieren.

Methodik. Bei 17 TEVAR-Patienten bestand folgende Indikation zur Konversion: retrograde Typ A Dissektion $(n=3)$, Typ Ia-Endoleak $(n=8)$, Typ Ib-Endoleak $(n=4)$, Stentgraftinfektion $(n=1)$, Aneurysma distal des Stentes $(n=1)$.

Die Behandlung umfasste je nach Ausmaß der Aortenpathologie den Ersatz der thorakoabdominellen oder thorakalen Aorta jeweils mit oder ohne Bogenersatz.

Bei 11 Patienten wurden die Stents komplett entfernt, in 6 Fällen wurden sie komplett oder partiell in die Aortenrekonstruktion miteinbezogen. Alle Konversionseingriffe erfolgten unter extrakorporaler Zirkulation, Liquordrainage und Neuromonitoring.

Ergebnisse. Alle Patienten überlebten die Operation. Die Mortalität betrug 17,6\%. Todesursachen waren: aortoösophageale Fistel ( $n=1$, Tag 51), septische Arrosionsblutung $(n=1$ Tag 24), Aortenklappenendokarditis $(n=1)$. Schweren Komplikationen beinhalteten 2 Paraparesen, 1 Nierenversagen, 1 Schlaganfall.

Schlussfolgerungen. Die offene Konversion nach TEVAR ist trotz der schwerwiegenden Pathologie mit akzeptabler Morbidität und Mortalität durchführbar. Die Mortalität ergibt sich vorwiegend aus septisch-infektiologischen Komplikationen.

\section{0}

\section{Biomechanical analysis of sternum support devices}

\section{Gorlitzer ${ }^{1}$, M. Bijak², M. Egger², S. Folkmann', E. Unger ${ }^{2}$, R. Moidl ${ }^{1}$, M. Grabenwöger ${ }^{1}$}

${ }^{1}$ Krankenhaus Hietzing, Vienna, Austria; ${ }^{2}$ Medizinische Universität Wien, Zentrum für Biomedizinische Technik und Physik, Vienna, Austria

Background. Based on the clinical success using the Posthorax support vest to prevent sternum related complications the biomechanical mechanism of different sternum support systems were evaluated.

Methods. Elastic bandages were compared with the Posthorax support vest in 27 patients after sternotomy and 27 volunteers. The effect of the supportive devices was acquired with eight special designed high sensitive real time pressure transducers, which were placed between chest wall and support devices. Measuring points during normal breathing, coughing and arm movement were analyzed. 
Results. The Postthorax support vest proved to be superior in the parasternal and posterior region comparing with the elastic bandage in all groups at normal breathing (Sensor $0+4: 2.788 \pm 0.79 \mathrm{~N}$ vs. $0.88 \pm 0.2 \mathrm{~N}$ and Sensor $3+7: 2.06 \pm 0.88 \mathrm{~N}$ vs. $0.78 \pm 0.04 \mathrm{~N} ; p=0.001$ ).

During coughing and arm movement all sensors at the anterior and posterior chest wall revealed a significant higher pressure using the Posthorax support vest (Sensor $0+4: 3.34 \pm 0.98 \mathrm{~N}$ vs. $1.47 \pm 0.59 \mathrm{~N}$; Sensor 3+7: 5.69 $\pm 2.99 \mathrm{~N} v s .1 .92 \pm 0.7 \mathrm{~N} ; p=0.001)$

Conclusions. The findings of the study proved a positive biomechanical effect of the Posthorax support vest in the anteroposterior movement which predicates its favourable clinical effect in avoiding sternum related complications.

\section{1}

\section{Chirurgie des zervikothorakalen Übergangs}

\section{W. Schreiner, O. Oster, M. Hanika, A. Zdrojek, H. Sirbu}

Thoracic Surgery, University Hospital, Erlangen, Germany

Grundlagen. Die Tumore im Bereich der oberen Thoraxapertur sind unabhängig von ihrer Äthiologie selten. Die enge anatomische Beziehung zur neurovaskulären Achse, der Wirbelsäule und der Thoraxwand stellt besondere Ansprüche an die chirurgische Sanierung. Der operative Zugang zur dieser Region durch den Zugang nach Shaw oder einer Reihe anteriorer Zugänge erreicht wird. In dieser Arbeit werden unsere Ergebnisse nach operativen Sanierung benigner und maligner Tumore im Bereich der Thoraxapertur zusammengefasst.

Methodik. Im Zeitraum 07.2007-10.2010 wurden 16 Patienten operiert. In 4 Fällen wurde ein DartevelleZugang oder die Modifikation, in je 6 Fällen eine Hemiclamshell-Inzision und Shaw-Paulson-Zugang gewählt.

Ergebnisse. In 7 Fällen wurde die Rippenresektion (1,4 \pm 1,3 Rippen) durchgeführt. Zwei Patienten benötigten den Thoraxwandersatz mit Marlexpatch. In zwei Fällen erfolgte Laminektomie. In allen Fällen wurde die R0Resektion erzielt. In zwei Fällen trat die Wundheilungstörung auf. Eine Plattenosteosynthese nach Klavikulotomie benötigte eine Revision bei Instabilität. In zwei Fällen wurde leichte Mobilitätseinschränkung im Schultergelenk festgestellt.

Schlussfolgerungen. Der operative Zugang wird unter der Berücksichtigung der genauen Tumorlokalisation und seiner Ausdehnung als auch der anatomischen Beziehung zur den Nachbarstrukturen gewählt. Durch die Wahl des optimalen Zugangs kann den besonderen anatomischen Anforderungen an die operative Sanierung gerecht werden.

\section{Arbeitsgemeinschaft für Chirurgische Endokrinologie 1: Incidentalom der Schilddrüse. Wann observieren? Wann operieren? Wie operieren?}

\section{2}

\section{Almost $50 \%$ of thyroid nodules are "incidentaloma"}

\section{G. Wolf, D. Malliga, H. Cerwenka}

Surgical Clinic, Graz, Austria

Background. Due to emerging Health Screening Programs, thyroid ultrasound is performed generally in all patients. Subclinical thyroid nodes get dedected, the vast majority in younger patients.Further screening dedects a new proportion of indications for operation.

Methods. The preclinical/preoperative course of all patients from 2000 to 2009 was evaluated. Those without clinical signs, without overt complaints,dedected only by sonography, where considered "incidentaloma".

Results. Those eligible for surgery counted for about $10 \%$ of all those diagnosed. Mostly, cold nodules,suspicious or inconclusive FNAP, or progredient size where operated, endocrine disorders counted for only $15 \%$. About $60 \%$ where nodes between 1 and $2 \mathrm{~cm}$, and $30 \%$ larger limbs, but yet unknown to the patient. All cases of thyroid cancers in this group (all Tla or Tlb) had a correct or suspicious FNAP, or where minimal-invasive follicular cancers, all less than $2 \mathrm{~cm}$.

Conclusions. Thyroid Ultrasound is performed regularely in Health Screening Programs, so subclinical nodes get detected very early. The vast majority can be observed, but further evaluation reveals a considerable number of very early diagosed thyroid cancers (pTla-b) that can be treated less radical. Minimal non-malignant, progredient nodes can be treated minimally-invasive way before emerging in a clinical state.

\section{3}

Multifocality of incidental papillary microcarcinoma of the thyroid - there is no evidence for increased malignant potential

M. Adambegan ${ }^{1}$, E. Gschwandtner ${ }^{1}$, N. Neuhold ${ }^{2}$, K. Tonninger ${ }^{1}$, A. Schultheis ${ }^{2}$, F. Kober ${ }^{1}$, M. Hermann ${ }^{1}$

${ }^{1}$ Department of Surgery, Kaiserin-Elisabeth-Spital der Stadt Wien, Vienna, Austria; ${ }^{2}$ Department of Pathology and Bacteriology, Kaiserin-Elisabeth-Spital der Stadt Wien, Vienna, Austria 
Background. According to present guidelines, postoperative finding of unifocal papillary microcarcinoma does not require completion thyroidectomy and radioiodine treatment. In contrast, multifocality obligates radical therapeutical concept, however, evidence based data do not exist.

Methods. We analysed histopathological features of patients with papillary microcarcinoma (PMC) treated in Kaiserin Elisabeth Hospital Vienna, and studied the clinical outcome in a follow-up period of up to 35 years.

Results. Incidental PMC was diagnosed in 754 patients. Only 5 PMC presented preoperatively with clinically apparent lymph node metastases. Multifocality was seen in $31 \%$, extra thyroidal extension in $2 \%$ of the tumours. $23 \%$ received radioiodine therapy. Only three patients, who all presented with clinically suspect cervical nodes at initial surgery, experienced recurrence in cervical lymph nodes and underwent successful re-operation. No patient died of the disease.

Conclusions. In incidentally detected PMC, multifocality per se is not a risk factor for a more aggressive course; therefore, we do not recommend completion thyroidectomy and radioiodine treatment. However, PMC detected due to clinically suspect and histologically confirmed lymph node metastases may show a more aggressive course with disease recurrence despite radical therapeutical concept. We recommend implementing a routine cervical lymph node sonography in thyroid diagnosis prior to thyroid surgery.

\section{4}

\section{Thyroid carcinoma in patients with} Hashimoto's thyroiditis: a retrospective analysis

\section{T. Resch, J. Pratschke, R. Prommegger}

Universitätsklinik für Visceral-, Transplantationsund Thoraxchirurgie Innsbruck, Innsbruck, Austria

Background. The role of Hashimoto's thyroiditis (HT) as a risk factor for the development of thyroid cancer as well as its prognostic significance remain controversial.

Methods. Between 06/2006 and 10/2010, 27 patients with HT associated thyroid cancer were operated on at the surgical department of Innsbruck Medical University Hospital and were retrospectively analysed.

Results. Twenty-three female and 4 male patients (24-69 yrs, mean 43) were diagnosed with thyroid cancer following thyroidectomy, Follow up ranged from 3.5 to 36 (mean 18.4) months. All tumors presented as papillary thyroid carcinomas (PTC) (follicular variant: 8, papillary tall cell variant: 2, 1 with signs of anaplastic transformation). $26 \%$ ( $n=7$ ) were detected intraoperatively by frozen section analysis (59\% not detected, 4 cases: not performed). The majority of patients presented at an early tumor stage (pT1a: 14, pT1b: 5, pT2: 2, pT3: 5, pT4:1; mean diameter $=6.9 \mathrm{~mm}$ ), $40 \%$ were multifocal, $22 \% \mathrm{N1}$; $0 \% \mathrm{M} 1$. Cancer recurrence occured in 3 cases. $62 \%$ received adjuvant radioactive iodine therapy. None of the patients died from tumor progression.

Conclusions. Although most HT-associated carcinomas were diagnosed at an early tumor stage, unfavourable tumor histology and recurrence rate are high.

\section{5}

\section{Rezidivstrumektomie nach primär benigner Schilddrüsenerkrankung}

\section{Sperker, A. Abrahim, M. Kees, M. Pramhas, M. Schermann, R. Roka}

Krankenanstalt Rudolfstiftung, Wien, Austria

Grundlagen. Unklare anatomische Verhältnisse durch Vernarbungen oder komplikationsbedingten Verlauf der Erstoperation stellen hohe Anforderungen an den Chirurgen bei Eingriffen an Rezidiv Strumen. Als Leitprinzip gilt maximal notwendige Radikaliät bei minimaler Komorbidität.

Methodik. Untersucht wurden alle Patienten, die in den Jahren 2000 bis 2010 aufgrund einer Rezidivstruma bei primär benigner Histologie operiert wurden.

Ergebnisse. Insgesamt wurden 147 Patienten aufgrund einer Rezidivstruma operiert. Die Rate einer passageren Rekurrensparese lag bei 6,8\% (Literatur: 0-22\%), eine permanente Rekurrensparese trat bei 3,4\% (Literatur: $0-13 \%$ ) der Patienten auf. Hypocalciämien bedingt durch Schädigung der Nebenschilddrüsen traten bei 8,8\% passager und bei 2,7\% der Patienten permanent auf (Literatur: $0-22 \%$ )

Schlussfolgerungen. Die besonderen Anforderungen bei intrathorakaler Lage sowie technische Empfehlungen werden diskutiert. Das Neuromonitoring des Nervus laryngeus recurrens ist eine essentielle Hilfe zum Auffinden des Nervens und kann bei kontinuierlicher Überprüfung zur intraoperativen Entscheidungshilfe das Resektionsausmaß betreffend dienen. Auch die intraoperative Überwachung des Parathormonspiegels könnte in Zukunft einen höheren Stellenwert erlangen, wenn klare Grenzwerte definiert werden können. Dabei muss auf „missed Glands“ geachtet werden, da die Gefahr eines Hypoparathyreodismus durch Nebenschilddrüsen-Autotransplantation deutlich verringert werden kann. 


\section{Arbeitsgemeinschaft für Endoskopie in der Chirurgie: Neues und Bewährtes in der chirurgischen Endoskopie}

\section{9}

„Tailor Made“ Therapiekonzept für Zenker Divertikel - Erfahrungswerte der letzten 10 Jahre

\section{A. Abrahim, C. Sperker, S. Ali-Abdullah, R. Roka}

Krankenanstalt Rudolfstiftung, Wien, Austria

Grundlagen. Eine chirurgische Sanierung des Zenker Divertikels ist bei Auftreten von klinischen Beschwerden sinnvoll, jedoch stellt sich die Frage nach dem optimalen Verfahren.

Methodik. Es wurden über den Zeitraum von mehr als 10 Jahren (2000-2010) die Daten von allen Patienten $(n=51)$ mit chirurgisch therapiertem Zenker Divertikel ausgewertet. Durchgeführt wurden entweder die transorale Schwellendurchtrennung $(n=45)$ oder der offene Zugang im Sinne einer Zervikotomie $(n=6)$. Ein modifizierter hauseigener Score wurde prä-, frühpost- und spätpostoperativ erhoben, um die klinische Effektivität der jeweiligen Methode zu objektivieren.

Ergebnisse: Die Patienten wurden im Durchschnitt 35,3 Monate einer Nachsorge unterzogen. Unseren Ergebnissen entsprechend, haben sich hinsichtlich des erhobenen Scores unabhängig vom operativen Verfahren, eine frühpostoperative Beschwerdefreit von $82,2 \%$ und eine spätpostoperative Beschwerdefreiheit von 55,6\% ergeben. Die transorale Schwellendurchtrennung zeigt eine signifikant kürzere postoperative Aufenthaltsdauer (transorale Schwellendurchtrennung Mittel: $4 \mathrm{~d} /$ Zervikotomie Mittel: $9,83 \mathrm{~d} / p=0,008)$. Die Entwicklung eines Zenker-Rezidivs in Abhängigkeit des operativen Verfahrens, fällt für die offene Methode günstiger aus (transorale Schwellendurchtrennung Mittel: 0,09/Zervikotomie $0 / p=0,044$ ). Die postoperativen Komplikationen sind vergleichbar.

Die transorale Schwellendurchtrennung besticht durch die kurze postoperative Aufenthaltsdauer und die geringe Invasivität.

Schlussfolgerungen. Unter Berücksichtigung des klinischen Scores zeigen beide Methoden eine hohe Effizienz. Vom rein morphologischen Aspekt und der Rezidivwahrscheinlichkeit erzielt jedoch die Zervikotomie postoperativ die besseren.

\section{0}

\section{Transrectal Single Port Approach: an alternative to NOTES, TEM or ESD}

\author{
C. Mittermair, W. Brunner, J. Schirnhofer, R. Frass, \\ N. Waldstein, K. Pimpl, C. Obrist, H. Weiss
}

KH Barmherzige Brüder, Salzburg, Austria

Background. For the past 25 years development of both, transanal and endoscopic techniques for resection of local tumours in the rectum have been established. The technique of single port surgery allows an alternate approach utilizing laparoscopic instruments for endolumenal colonic tumour resection. Herein we present the technical aspects of this novel method.

Methods. In November 2010 we operate on two male patients suffering from benign tumours in the mid and low rectum. In both cases endoscopic snare resection was not feasible for length and orientation of the lesion. Patients were placed in lithotomy position and a SILS port (Covidien) was fixed transanally. Using intralumenal carbon-dioxide insufflation, two working instruments and a five millimetre $30^{\circ}$ camera were delivered through the port. Submucosal preparation was carried out by means of ultrasonic or monopolar electrocauteric dissection. No closure of the mucosal defect was necessary.

Results. Both resections were completed successfully (120 and $60 \mathrm{~min}$ ), no intraoperative complication was observed. Entire continuity of dissection plane could be achieved. Histological evaluation revealed free resection margins, postoperative recovery of the patients were uneventful.

Conclusions. Transanal resection of large polyps using laparoscopic instruments can, in some cases, be a save and feasible alternative to NOTES, TEM, or ESD.

\section{1}

Ballonenteroskopie-gestützte ERCP als alternative Technik bei Patienten mit biliären Erkrankungen und postoperativ bestehender Roux-en-Y-Anastomose Progressionsbericht klinischer Altagserfahrungen an repräsentativer Fallserie einer bizentrischen systematischprospektiven Beobachtungsstudie

\section{P. Büschel ${ }^{1}$, K. Mönkemüller ${ }^{2}$, L. C. Fry ${ }^{2}$, P. Malfertheiner ${ }^{3}$, H. Lippert ${ }^{1}$, F. Meyer ${ }^{1}$}

${ }^{1}$ Universitätsklinikum Magdeburg A.ö.R., Universitätsklinik für Allgemein-, Viszeral- und Gefässchirurgie, Magdeburg, Germany; ${ }^{2}$ Marienhospital, Klinik für Innere Medizin,

Gastroenterologie \& Infektionskrankheiten, Bottrop, Germany; 
${ }^{3}$ Universitätsklinikum Magdeburg A.ö.R., Universitätsklinik für Gastroenterologie, Hepatologie und Infektiologie, Magdeburg, Germany

Grundlagen. Die endoskopische retrograde Cholangiopancreatographie (ERCP) ist technisch bei Patienten mit nachoperativen Rekonstruktionszuständen nach Oberbauchchirurgie anspruchsvoll. Im Sinne einer bizentrischen prospektiven Beobachtungsstudie wurde die technischen Erfolgsrate dieser Technik unter Zuhilfenahme der Ballon-Enteroskopie (BAE) untersucht.

Methodik. Prospektive Auswertung von konsekutiven Patienten mit Roux-en-Y-Anastomose, die eine BAEERCP erfuhren. Diagnostischer Erfolg wurde als erfolgreiche Gangkanülierung bzw. Diagnosesicherung angesehen, therapeutischer Erfolg wurde als erfolgreiche endoskopische Therapie der zugrunde liegenden Erkrankung angesehen.

Ergebnisse. Die BAE-gestützte ERCP wurde $44 \mathrm{mal}$ bei 32 Patienten (8 F, 24 M; mittleres Alter: 62,7) mit Roux-enY-Anastomose ( \pm Z.n. Hepaticojejunostomie) durchgeführt. Die Indikation zur ERCP umfasste: Cholangitis $n=10$, Gallensteine $n=9$; Cholestase $n=12$; Stententfernung $n=4$; postoperatives Galleleck $n=1$. Die diagnostische Erfolgsrate betrug $82 \%$, die therapeutische $78 \%$. Diagnostische Versagen: Keine Erreichbarkeit des proximalen Endes des afferenten Loops $(n=5)$. Therapeutisches Versagen: unmögliches Hochschieben des Führungsdrahtes in den Gallengang trotz adäquater transpapillärer Insertion des Katheters $(n=4)$, nicht mögliche Stentplatzierung trotz adäquat eingeführten Führungsdrahtes $(n=1)$. Es war lediglich eine Majorkomplikation zu verzeichnen (2,8\%): Perforation der Hepaticojejunostomie, welche letztlich chirurgisch saniert warden konnte.

Schlussfolgerungen. Die ERCP mittels BAE-gestütztem Vorgehen ist geeignet, auch Patienten mit komplexer „postoperativer“ Anatomie nach Oberbaucheingriffen im diagnostisch-therapeutischen endoskopischem Vorgehen in der überwiegenden Mehrheit der Fälle anzugehen und zu versorgen.

\section{2}

\section{Chirurgische Therapie des blutenden, peptischen Magenulkus im 21. Jahrhundert}

\author{
A. Dubecz ${ }^{1}$, K. Keller ${ }^{1}$, R. Stadlhuber ${ }^{1}$, \\ M. Schweigert ${ }^{1}$, H. Muschweck ${ }^{2}$, H. J. Stein ${ }^{1}$ \\ ${ }^{1}$ Klinikum Nürnberg, Klinik für Allgemein-, Viszeral- und \\ Thoraxchirurgie, Nürnberg, Germany; ${ }^{2}$ Klinikum Nürnberg, \\ Abteilung für Gastroenterologie, Nürnberg, Germany
}

Grundlagen. Auch wenn die aktuelle Literatur anderes vermuten lässt, ist die chirurgisch operative Versorgung der Goldstandard aller endoskopisch nicht kontrollierbaren blutenden, peptischen Magenulcera.
Methodik. In den Jahren 2004 bis 2009 unterliefen 64 Patienten einer chirurgisch operativen Versorgung eines blutenden, peptischen Magenulkus. Die Daten wurden retrospektiv ausgewertet und Nebenerkrankungen, OPIndikation, Operationsverfahren, vorangegangen endoskopische Verfahren, intraoperativer Befund, sowie perioperative Morbidität und Mortalität berücksichtigt.

Ergebnisse. Das Durchschnittsalter betrug 68 Jahre. $43 \%$ der Ulkusblutungen waren mit der Einnahme von Antikoagulanzien oder nichtsteroidaler Antiphlogistika vergesellschaftet. Bei allen Patienten wurde einer präoperative Gastroskopie durchgeführt. Der präoperative Hämoglobinwert betrug im Mittel 6,2 g/dl. Durchschnittlich erhielten die Patienten 9 Einheiten ErythrozytenKonzentrate. Bei $85 \%$ der Patienten fand sich das Ulkus im Duodenum. Die OP-Indikation war eine endoskopisch nicht kontrollierbare Blutung in 58 Fällen, in 6 Fällen ein erhöhtes Rezidivrisiko. Alle Patienten wurden postoperativ, durchschnittlich für 14 Tage, intensivmedizinisch betreut, bei einer Krankenhausmortalität von $23 \%$.

Schlussfolgerungen. Als unmittelbare Folge der weit fortgeschrittenen Möglichkeiten zur endoskopischen Ulkustherapie haben Patienten mit endoskopisch nicht kontrollierbaren Blutungen des oberen Gastrointestinaltrakts multiple Komorbiditäten und unterliegen einer hohen postoperativen Mortalität.

\section{Hauptsitzung - Komplikationsmanagement 6: Gallengangsverletzungen}

\section{4}

\section{Minimal invasive Therapie einer Läsion des Ductus choledochus nach laparoskopischer Cholecystektomie}

\author{
C. Dollinger ${ }^{1}$, K. Krafka ${ }^{1}$, J. Weber-Eibel ${ }^{2}$, \\ G. Fasching ${ }^{1}$ \\ ${ }^{1}$ Klinikum Klagenfurt, Abteilung für Kinder- und \\ Jugendchirurgie, Klagenfurt, Austria; ${ }^{2}$ Klinikum Klagenfurt, \\ 1. Medizinische Abteilung, Klagenfurt, Austria
}

Grundlagen. Das Risiko für eine Verletzung des Ductus choledochus nach laparoskopischer Cholecystektomie wird mit 0,4-0,6\% angegeben. Sie kann durch Transsektion, Lazeration, Clips, Durchblutungsstörung oder thermische Läsion verursacht sein.

Methodik. Eine 13 jährige Patientin wurde aufgrund von Gallensteinen laparoskopisch cholecystektomiert. Der Verlauf gestalteten sich komplikationslos, die Patientin wurde am 5. postoperativen Tag beschwerdefrei entlassen. 3 Tage später wurde sie aufgrund von Bauchschmerzen vorstellig, in der Sonographie 
zeigte sich zunehmend freie Flüssigkeit. Aufgrund der Symptomatik einer biliären Peritonitis wurde am 10. postoperativen Tag eine laparoskopische Revision durchgeführt, die ein Leck des Ductus choledochus in Nähe des Cysticus-Stumpfes zeigte. Aufgrund des nur kleinen Lecks entschied man sich zum Einlegen einer Drainage. Nach weiterem unzufriedenstellenden Verlauf mit persistierender Gallensekretion erfolgte eine ERCP mit Einlage eines Stents. Der weitere Verlauf war komplikationslos. Der Stent wurde nach 2 Monaten problemlos entfernt, die ERCP zeigte keinen Hinweis auf Striktur.

Schlussfolgerungen. Die Ursache der Läsion bei unserer Patientin ist nicht eindeutig geklärt. Wir vermuten eine thermische Schädigung durch monopolare Koagulation, die zur Nekrose der Wand des Ductus choledochus führte. Erste Symptome der biliären Peritonitis traten am 8. postoperativen Tag auf. Eine interne Ableitung mittels endoskopisch platziertem Stent kann auch bei Kindern erfolgreich angewandt werden.

\section{Hauptsitzung - Multimodale Konzepte}

\section{3}

Das Outcome neoadjuvant radiochemotherapierter Rektumkarzinome im Vergleich zu primär operierten Patienten - Ergebnisse eines onkologischen Schwerpunktkrankenhauses

\section{S. Stättner ${ }^{1}$, T. Braunschmid ${ }^{1}$, T. Hoblaj ${ }^{1}$, F. Bastian ${ }^{1}$,} M. Baur ${ }^{2}$, J. Karner ${ }^{1}$

${ }^{1}$ SMZ Süd, Kaiser Franz Josef Spital, Abteilung Chirurgie, Wien, Austria; ${ }^{2}$ SMZ Süd, Kaiser Franz Josef Spital, ${ }^{3}$ Med Abteilung, Zentrum für Hämatologie und Onkologie, Wien, Austria

Grundlagen. Die kombinierte Radiochemotherapie (RCT) ist bei lokal fortgeschrittenen Tumoren des mittleren und unteren Rektumdrittels vielzitierter Standard. Der hohen Rate an pathologischen Vollremissionen $(14,1 \%)$ und einer Sphinkter Erhaltungsrate über $80 \%$ sind eine erhöhte Morbidität und funktionelle Einschränkungen wie sexuelle Dysfunktion, Blasen- und Stuhlentleerungsstörungen entgegenzustellen.

Methodik. Die Ergebnisse einer prospektiv kontrollierten, einarmigen Phase II Studie zur neoadjuvanten Radiochemotherapie mit 75 ausgewerteten Patienten werden einer nicht vorbehandelten Vergleichsgruppe aus unserer prospektiv angelegten Datenbank colorektaler Karzinompatienten gegenübergestellt.

Ergebnisse. Neben schweren hämatologischen Nebenwirkungen (bis $27 \%$ ) traten im vorbehandelten
Kollektiv bei $5 \%(n=4)$ Anastomoseninsuffizienzen auf (bei einer protektiven Stomarate von 100\%), 1 Patient entwickelte eine Anastomosenstenose und 3 Patienten (4\%) eine erektile Dysfunktion. Die Lokalrezidivrate betrug $6,1 \%$ und das 5-Jahre erkrankungsfreie Intervall betrug 53,7\%. Diese Patienten werden mit mehr als 100 primär operierten Patienten ohne neoadjuvante Radiochemotherapie verglichen und ausgewertet.

Schlussfolgerungen. Langzeitdaten radiochemotherapierter Patienten mit Rektumkarzinom weisen auf eine signifikant höhere Morbiditätsrate und negative Beeinflussung der Lebensqualität hin. Die Indikationsstellung zur neoadjuvanten RCT sollte daher basierend auf standardisierter präoperativer Diagnostik kritisch in den lokalen Tumorboards diskutiert werden.

\section{4}

\section{Her-2 Expression beim gastroösophagealen Karzinom und seinen Metastasen}

\section{G. Jomrich, B. Jesch, F. Maroschke, P. Birner, S. F. Schoppmann}

Universitätstsklinik für Chirurgie, Wien, Austria

Ziel systemischer Krebstherapien die Behandlung klinischer und subklinischer Metastasen. Besonders zielgerichtete Therapien werden anhand der Analyse von Primärtumorgewebe indiziert. Beim gastroösophagealen Karzinomen ist die Konstanz der Her-2 Expression zwischen primärem Tumor und korrespondierenden Metastasen unbekannt. Bei 176 Patienten mit gastroösophagealen Karzinomen (97 Adenokarzinomen (AC) und 79 Plattenepithelkarzinomen (SCCs)) wurden die Her-2 Expressions bzw Hybridisierungmuster des Primärtumors und deren Metastasen immunhistochemisch und mittels dual colorimetric in situ hybritization untersucht. HER-2 status positv wurden $14,4 \%$ der ACs und 1,3\% der SCCs identifiziert. Bei 95,3\% der ACs und 98,6\% der SCCs konnte ein identischer HER-2 Statuts zwischen dem Primärtumor und Lymphnotenmetastase festgestellt werden (sign. mit $p>0,05$ ). 86,3\% der AC-Fernmetastasen zeigten einen identen HER-2-Status verglichen mit dem Primärtumor. Dies konnte auch bei $100 \%$ der SCCs gezeigt werden. 9,1\% der HER-2 negativen ACs entwickelten HER-2 positive Metastasen.Eine neoadjuvante Chermotherapie hatte keinen Einfluss auf den HER-2 Status. Aufgrund einer $85 \%$-igen Konkordanz des HER-2 Status kann eine routinemäßige HER-2 Testung aus Metastasengewebe als Bestätigung eines positiven HER-2 Status nicht generell empfohlen werden. Eine sinnvolle Option kann allerdings eine Beurteilnung des HER-2 Status der Metastasen bei Patienten mit leicht zugänglichen Metastasen, zuvor insuffizienter oder überhaupt fehlender Analyse des Primärtumors darstellen. 


\section{5}

\section{Simultaneous detection of frequent KRAS mutations and BRAF V600E in FFPE colorectal tissue samples}

\author{
V. Buxhofer-Ausch ${ }^{1}$, G. Kriegshäuser ${ }^{1}$, C. Oberkanins ${ }^{2}$, \\ B. Rauscher ${ }^{2}$, R. Zeillinger ${ }^{1,2}$, C. Ausch ${ }^{1,3}$ \\ ${ }^{1}$ Cluster für Translationale Onkologie Ludwig Boltzmann \\ Forschungsgesellschaft, Wien, Austria; ${ }^{2}$ ViennaLab, Wien, \\ Austria; ${ }^{3}$ Chirurgische Abteilung Donauspital am SMZ Ost, \\ Wien, Austria
}

Background. Patients with mutant BRAF V600E colorectal tumors show impaired responsiveness to cetuximab and panitumuomab therapy. Since KRAS and BRAF mutations are mutually exclusive in colorectal cancer (CRC), the BRAF V600E mutation further empowers the selection of eligible patients for EGFR-antibody treatment. We report here a novel reverse-hybridization $(\mathrm{RH})$ assay for the combined detection of frequent KRAS mutations and the BRAF V600E mutations in formalin-fixed paraffin-embedded (FFPE) colorectal tissue samples.

Methods. An existing RH assay for the simultaneous detection of 10 mutations in codons 12 and 13 of the KRAS gene was modified to contain BRAF mutation V600E. The modified RH assay was then used to analyze FFPE tissue samples obtained from 74 CRC patients.

Results. Twentysix (35\%) and $2(1.5 \%)$ of the samples contained a mutation in KRAS and BRAF, respectively. The results were confirmed by DNA sequencing (dideoxy method).

Conclusions. The RH assay test proofed to be a sensitive tool for the simultaneous detection of KRAS/BRAF mutations in archived FFPE tissue samples.

\section{6}

\section{Neoadjuvant chemotherapy increases breast conservation in lobular type breast cancer patients}

\section{F. Fitzal ${ }^{1}$, M. Mittlböck ${ }^{2}$, G. Steger ${ }^{3}$, R. Bartsch ${ }^{3}$,} M. Rudas ${ }^{4}$, P. Dubsky ${ }^{1}$, O. RiedI ${ }^{5}$, R. Jakesz ${ }^{1}$, M. Gnant ${ }^{1}$

${ }^{1}$ Chirurgie, Wlen, Austria; ${ }^{2}$ Statistik, Wlen, Austria; ${ }^{3}$ Innere Medizin I Onkologie, Wlen, Austria; ${ }^{4}$ Pathologie, Wlen, Austria; ${ }^{5}$ Chirurgie, Krems, Austria

Background. Our study aims to determine whether patients with lobular type breast cancer should undergo neoadjuvant chemotherapy (nCT).

Methods. Patients who received nCT and surgery between 1995 and 2007 at the Medical University Vienna were retrospectively analysed.

Results. 325 patients had a median follow up of 53 months. $21 \%$ had lobular cancer, $70 \%$ of them were ini- tially scheduled for mastectomy (MX). 21 finally received breast conservation (BCT) yielding a MX-BCT turnover rate of $45 \%$. $20 \%$ of the patients primarily scheduled for BCT had to finally undergo MX in lobular cancer. The 256 patients with ductal type breast cancer finally had a MXBCT turnover rate of $52 \%(p=0.561 v s$. lobular $)$ and a BCT-MX turnover rate of $15 \%$ ( $p=0.933 v s$. lobular). Secondary MX after initial BCT was necessary in $2 \%$ (ductal) and $10 \%$ (lobular; $p=0.110$ ), respectively. There was no difference in local recurrences in lobular compared with ductal type breast cancer patients after BCT $(2.7 \%$ versus $10 \% p=0.135$ ) as well as in lobular breast cancer patients comparing BCT with MX (2.7\% versus $3.4 \% p=0.795)$. Tumor type was neither an independent predictor for BCT nor local recurrence.

Conclusions. Systemic Chemotherapy may increase BCT rate in lobular type breast cancer.

\section{7}

\section{Gastrointestinale Stromatumore - eine retrospektive single center Analyse}

\author{
I. E. Kronberger ${ }^{1}$, Q. D. Chalupar ${ }^{2}$, R. Kafka-Ritsch ${ }^{1}$, \\ J. Pratschke ${ }^{1}$, M. Zitt ${ }^{1}$ \\ ${ }^{1}$ Univ.-Klinik für Visceral-, Transplantations- und \\ Thoraxchirurgie, Innsbruck, Austria; ${ }^{2}$ Medizinische \\ Universität Innsbruck, Innsbruck, Austria
}

Grundlagen. GIST repräsentieren die häufigsten mesenchymalen Tumore im Gastrointestinaltrakt, dennoch handelt es sich um eine seltenes Krankheitsbild, welches erst seit wenigen Jahren mehr Aufmerksamkeit im klinischen Alltag erhält.

Methodik und Ergebnisse. Alle Patienten, die seit 1997 aufgrund eines GIST an unserer Abteilung operiert wurden, werden retrospektiv analysiert. Bis Dezember 2004 waren bei 31 Patienten (19 Männer, 12 Frauen) die klinischen Daten erhebbar. Das mittlere Alter zum Diagnosezeitpunkt betrug 60 Jahre $( \pm 11)$. Primäre GIST des Magens traten in $58 \%$ (18 Patienten, davon 61 \% Männer) auf, $20 \%$ der Tumore kamen im Dünndarm und $10 \%$ im Duodenum vor. Nur 2 Patienten zeigten eine primäre kolorektale Lokalisation. Zum Diagnosezeitpunkt zeigen sich Tumorgrößen bis zu $18 \mathrm{~cm}$ Durchmesser (Median $6 \mathrm{~cm}$ ) - bei einem Drittel der Patienten kann bereits eine Fernmetastasierung gefunden werden. Histologisch erweist sich der spindelzellige Typ bei 21 Patienten (68\%) neben dem epitheloiden Typen (19\%) als der häufigste.

Schlussfolgerungen. Tyrosinkinaseinhibitoren sind seit wenigen Jahren eine neue Option neben der operativen und noch immer rezidivträchtigen Therapie. Aufgrund der rezenten Neuerungen stellen wir mit dieser Studie einen Vergleich zwischen der Patientengruppe bis 2004 und der gerade in Auswertung befindlichen Gruppe ab 2005 an. Dabei werden sowohl histopathologische und genetische als auch klinische Parameter analysiert. 


\section{8}

Retrospective 10 year analysis of 37 patients with anal carcinoma - Outlook and recommendations for a standardized treatment

\section{May ${ }^{1}$, M. Brunialti ${ }^{1}$, K. S. Glaser ${ }^{1}$, C. Stanek ${ }^{2}$}

${ }^{1}$ 2. Department of General Surgery, Wilhelminenspital, Wien, Austria; ${ }^{2}$ Department of Radiooncology, Wien, Austria

Background. Carcinoma of the anus is a rare malignancy representing $1.5 \%$ of all carcinomas of the gastrointestinal tract with an increasing rise in incidence in the last 25 years. One of the main challenges is the correct and early diagnosis which is often delayed causing a potential reduction of curative success. There has been a dramatic change in therapeutic approach 20 years ago switching from surgical excision to combined chemoradiotherapy.

Methods. We have reviewed and followed-up 37 patients (33 female, 4 male) with an average age of $68 \pm$ 12 years presenting with anal cancer that have been seen by our outpatient clinic in the last 10 years and analysed factors regarding survival, quality of life (including degree of continence, chronic skin lesions, SF36), the need for surgery like salvage abdominoperineal resection (APRE).

Results and conclusions. Based on this retrospective analysis recommendations for a standardized treatment will be presented.

\section{Arbeitsgemeinschaft für Chirurgische Endokrinologie 2: Primärer Hyperparathyreoidismus}

\section{9}

Minimally invasive parathyroidectomy without QPTH monitoring in an endemic goiter region

\section{P. Riss ${ }^{1}$, C. Scheuba ${ }^{1}$, R. Asari ${ }^{1}$, C. Bieglmayer ${ }^{2}$, B. Niederle}

${ }^{1}$ Med. Universität Wien, Univ. Klinik für Chirurgie, Sektion Chirurgische Endokrinologie, Wien, Austria; ${ }^{2}$ Med. Universität Wien, Klin. Institut für Med. und chem. Labordiagnostik, Wien, Austria

Background. It is matter of discussion if quick parathyroid hormone (QPTH)-monitoring is helpful in patients with primary hyperparathyroidism (PHPT) and "localized single gland disease" (SGD) to further increase the rate of success performing minimally invasive parathyroidectomy (MIP). The aim of this study was to evaluate, if a randomized controlled trial was justified in order to clarify this discussion.

Methods. The prospective database of patients with sporadic PHPT, SGD, MIP and QPTH-monitoring was evaluated regarding the "conversion rate" to bilateral exploration and permanent normocalcemia ("QPTH"group). The patients were re-analyzed a second time "without" applying QPTH-monitoring ("Non-QPTH"group).

Results. By definition, 338 patients with "localized SGD" underwent MIP. MIP was finished in 308 (91.1\%) patients. 3 of 308 patients $(0.9 \%)$ showed persisting disease

In 30 of 338 patients $(8.9 \%)$ a conversion to bilateral exploration was necessary. Analyzing the "Non-QPTH"group, 14 additional patients showed persisting disease. Thus, without using QPTH- monitoring, the rate of persisting PHPT would increase from $0.9 \%$ (3 patients) to $5.0 \%$ (17 patients; $p=0.0005$ ).

Conclusions. Intraoperative QPTH-assay seems necessary even in patients with "localized SGD" in an endemic goiter region. Abandoning QPTH-monitoring would more than double the rate of persisting disease. A randomized trial seems not to be justified.

\section{0}

\section{Thyreoidektomie und Darstellungspflicht der Nebenschilddrüsen}

\author{
C. Bures ${ }^{1}$, L. Preldzic ${ }^{1}$, S. Göbl ${ }^{1}$, R. Promberger ${ }^{2}$, \\ J. Ott ${ }^{2}$, F. Kober ${ }^{1}$, M. Hermann ${ }^{1}$ \\ ${ }^{1} \mathrm{KES}$, Wien, Austria; ${ }^{2} \mathrm{AKH}$ Wien, Wien, Austria
}

Grundlagen. Nach wie vor gibt es keine evidenzbasierten Daten, wie viele Nebenschilddrüsen zur Vermeidung eines dauerhaften Hypoparathyreoidismus bei einer Thyreoidektomie dargestellt werden müssen.

Methodik. Bei prospektiven Erfassung der dargestellten Anzahl der Nebenschilddrüsen wurden nach abgeschlossener Nachuntersuchung von insgesamt 543 Patienten nach totaler oder fast totaler Thyreoidektomie 4 Gruppen unterschieden: A: permanent manifester Hypoparathyreoidismus (PTH und Ca pathologisch/Substitution), B permanent latenter Hypoparathyreoidismus (PTH normal, Ca pathologisch/Substitution), C postoperativer passagerer Hypoparathyreoidismus (restitutio ad integrum), D Kontrollgruppe ohne Hypoparathyreoidismus.

Ergebnisse. In Gruppe A wurden durchschnittlich 2,1 Nebenschilddrüsen dargestellt, bei Gruppe B 2,2, in Gruppe C 2,6, in der Kontrollgruppe D durchschnittlich 2,1 . 
C war jeweils im Vergleich zu A, zu B und zu D signifikant erhöht, keine Signifikanz ergab sich zwischen A und $\mathrm{B}, \mathrm{A}$ und $\mathrm{D}, \mathrm{B}$ und $\mathrm{D}$.

Schlussfolgerungen. Die höchste Anzahl an NSD im Operationsfeld wurde in der Gruppe des postoperativ passageren Hypoparathyreoidismus gesehen. Zwischen Patienten ohne Komplikation und jenen mit permanenten Hypoparathyreoidismus war kein Unterschied zu verzeichnen. Die Forderung nach einer definierten Anzahl aufzufindender Nebenschilddrüsen kann nicht aufrechterhalten werden. Der Unterschied liegt möglicherweise in einer individuell unterschiedlichen Erkennung bzw. Präparationstechnik der Epithelkörperchen. Wesentlich ist nicht, WIEVIELE Nebenschilddrüsen GESEHEN werden, sondern dass keine ÜBERSEHEN ODER DEVASKULARISIERT werden.

\section{1}

\section{Kann die CT-MIBI-SPECT Bildfusion zur Lokalisationsdiagnostik des primären Hyperparathyreoidismus dessen Mehrdrüsenerkrankung vorhersagen?}

\author{
R. Prommegger ${ }^{1}$, G. Wimmer ${ }^{1}$, R. Bale ${ }^{2}$, D. Putzer ${ }^{3}$, \\ T. Resch ${ }^{1}$, J. Pratschke ${ }^{1}$ \\ ${ }^{1}$ Abteilung für Viszeral-, Transplantations-, und \\ Thoraxchirurgie, Innsbruck, Austria; ${ }^{2}$ Univ. Klinik \\ für Radiologie, Innsbruck, Austria; ${ }^{3}$ Abteilung für \\ Nuklearmedizin, Medizinische Universitätsklinik Innsbruck, \\ Innsbruck, Austria
}

Grundlagen. Für die minimal invsive Operation des primären Hyperparathyreoidismus ist eine präoperative Lokalisationsdiagnostik notwendig. Die CT-MIBI-SPECT Bildfusion (virtuelle Halsexploration) erfüllt diese Voraussetzung in hohen Maß. Ziel dieser Untersuchung war es ob durch die CT-MIBI-Spect Bildfusion die Mehrdrüsenerkrankung beim primären Hyperparathyreoidismus präoperativ diagnostizierbar ist.

Methodik. Bei 116 Patienten mit primärem Hyperparathyreoidismus wurde die CT MIBI SPECT Bildfusion durchgeführt. Mit durch eine Vakuummatratze fixierten Kopf und Hals wurde einerseits eine Computertomographie und anderseits ein MIBI-SPECT durchgeführt. Mit einer speziellen Software wurden die Bilder fusioniert und an einer Workstation betrachtet. Wenn mindestens zwei Drüsen erkennbar waren wurde eine Mehrdrüsenerkrankung diagnostiziert.

Ergebnisse. Bei 6 von 116 Patienten $(5,7 \%)$ wurde spätestens intraoperativ eine Mehrdrüsenerkrankung diagnostiziert. In 5 dieser 6 Patienten konnte die Mehrdrüsenerkrankung bereits präoperativ durch die Bildfusion diagnostiziert werden indem mindestens 2 Drüsen dargestellt werden konnten.

Schlussfolgerungen. Die CT-MIBI SPECT Bildfusion erlaubt in hohem Maße die Mehrdrüsenerkrankung des primären Hyperparathyreoidismus präoperativ zu diagnostizieren. Dies könnte unter Umständen die aufwändige intraoperative PTH Bestimmung ersetzten und auf familiäre Formen des primären Hyperparathyreoidismus hinweisen.

\section{Hauptsitzung - Chirurgie in den Medien}

\section{6}

\section{Plastische, Ästhetische und \\ Rekonstruktive Chirurgie - Ist Google eine Quelle für ausgewogene Information?}

\section{H. Koch, M. Binder}

Klinische Abteilung für Plastische, Ästhetische und Rekonstruktive Chirurgie, Medizinische Universität Graz, Graz, Austria

Grundlagen. Das Internet ist ein wichtiges Informationsmedium für Patient_innen. Die Seriosität und Qualität medizinischer Informationen im Internet ist jedoch unreglementiert. Ziel der Studie war, Informationen aus dem Bereich der Plastischen Chirurgie im Internet zu analysieren.

Methodik. Über die Suchmaschine Google wurden Abfragen nach Begriffen aus den Bereichen Wiederherstellungschirurgie, ästhetische Chirurgie und Handchirurgie durchgeführt. Die jeweils ersten 500 Ergebnisse pro Begriff wurden einer Analyse unterzogen. Diese umfasste zunächst die Informationsquelle. Zusätzlich wurde die Tiefe der Information analysiert. Erhoben wurde, inwieweit auf verschiedene Operationstechniken sowie auf mögliche Komplikationen und die Nachbehandlung eingegangen wurde.

Ergebnisse. Von den ausgewerteten Seiten stammten etwa $25 \%$ von ÄrztInnen, der Rest von unterschiedlichen anderen Anbietern. Verschiedene Operationstechniken in den einzelnen Feldern wurden in deutlich unter $50 \%$ der ärztlichen Internetangebote erwähnt. Mögliche Komplikationen wurden in deutlich weniger als einem Drittel der Fälle erwähnt, die Nachbehandlung wurde in etwa der Hälfte der Fälle erläutert.

Schlussfolgerungen. Ärztliche Informationen zum Thema Plastische Chirurgie im Internet beinhalten tendenziell positive Aspekte. Potentielle Komplikationen werden eher verschwiegen, Nachbehandlungsschemata nur teilweise erwähnt. Unsere Studie zeigt, dass das Internet ärztlicherseits in erster Linie als Marketinginstrument gesehen wird. Da es von Laien als wichtige Informationsquelle genutzt wird, bleibt zu diskutieren, ob von ärztlichen AnbieterInnen eine ausgewogenere Darstellung medizinischer Inhalte zu fordern wäre. 


\section{POSTER}

\section{Adipositaschirurgie}

\section{P01}

\section{Lagerungsbedingte Rhabdomyolyse bei einem Patienten mit Adipositas permagna nach laparaskopischer Magen Sleeve Resektion}

\section{Wielandner, F. Birkfellner, H. Nehoda}

BKH St. Johann, St. Johann in Tirol, Austria

Grundlagen. Aktueller Fallbericht eines adipösen Patienten (BMI 42) mit postoperativer Rhabdomyolyse mit beginnendem Kompartmentsyndrom des Musc. glutaeus maximus nach laparaskopischer SleeveResektion.

Methodik. Standardmäßig wurde er für einen Tag an unserer ICU überwacht. Nach Rückverlegung auf die Normalstation entwickelte der Patient eine zunehmend schmerzhafte Schwellung im Bereich der linken Gesäßhälfte. Unter forcierter Diurese und antiphlogistischer Therapie sowie entsprechender Lagerung kam es zur Abschwellung. Eine chirurgische Intervention war nicht nötig. In den durchgeführten Laborkontrollen zeigte sich eine maximale Auslenkung der Kreatinkinase auf $12.000 \mathrm{U} / \mathrm{l}$. Die Nierenfunktionsparameter stiegen nie an.

Ergebnisse. Der Patient konnte beschwerdefrei aus der stationären Behandlung entlassen werden. Erfolgreicher Gewichtsverlust von $43 \mathrm{~kg}$ innerhalb eines Jahres. Normale Nierenfunktion.

Schlussfolgerungen. Die Rhabdomyolyse ist eine häufige Ursache des akuten Nierenversagens, meist verursacht durch Ischämie/Reperfusion oder ein traumatisches Ereignis. Aktuelle Studien in der bariatrischen Chirurgie verweisen auf ein erhöhtes Risiko adipöser Patienten zur Entwicklung eines lagerungsbedingten Kompartment-Syndroms mit daraus resultierenden Nierenversagens. Ein erhöhtes Risiko haben vor allem männliche Patienten mit ausgeprägter Adipositas und einer langen OP-Dauer.

Unser Patient hatte einen BMI deutlich unter der in der Literatur beschriebenen Risikogruppe. Trotzdem entwickelte er ein gluteales Kompartment-Syndrom. Nephroprotektive Maßnahmen mit rasch begonnener Diurese und antiphlogistischer Behandlung waren erfolgreich.

\section{P02}

Aortendissektion Typ B - eine bislang in der internationalen Literatur nicht beschriebene Komplikation der laparoskopischen MagenbandImplantation. Ein Fallbericht über Komplikation und Management

\section{O. Riedl, S. Sattler, W. Lechner, R. Ciovica, M. Gadenstätter}

Abteilung für Allgemeinchirurgie, Landesklinikum Krems, Krems, Austria

Grundlagen. Bei der laparoskopischen Magenbandimplantation ist der kritische Schritt die Bildung des Bandkanals hinter dem gastro-ösophagealen Übergang. Dies erfolgt ohne Sichtkontrolle durch stumpfe Präparation mittels eines Dissektionsinstruments („Goldfinger“). Die Hinterwand dieses Kanals wird durch den linken Zwerchfellschenkel gebildet. Hier besteht eine unmittelbare Nähe zur Aorta.

Methodik. 42 jährige Patientin. BMI 41. Internistische OP-Freigabe. Intraoperativer Verlauf unauffällig. $2 \mathrm{~h}$ Aufwachraum. Übernahme auf Normalstation. $3 \mathrm{~h}$ postoperativ Parästhesien und Schmerzen im linken Bein; progredient trotz hochdosierter analgetischer Therapie. Schließlich das schmerzhafte Bein kühler.

Diagnostik: Farb-Duplex-Sonographie: Verdacht auf Gefäßverschluss linke Beckenetage. Kontaktaufnahme mit Gefäßchirurgie. Transferierung. CT-Angiographie: Aortendissektion Typ B mit komplettem Verschluss der A. iliaca comm. sin.; Dissektionshöhe Th10.

Therapie: Stentgraft-Implantation supracöliakal; mehrfache Stentimplantation linke Beckenetage. Postinterventionell die Viszeralgefäße offen, Beckenetage frei.

Ergebnisse. Chronisches Schmerzsyndrom, Sensibilitätsstörungen, inkomplette Peronaeusparese links.

Schlussfolgerungen. (1) Aortendissektion vermutlich durch Druck mit Goldfinger bei ev. Vorläsion; (2) dieses „stumpfe Gefäßtrauma“ führte nicht zur Blutung und blieb intraoperativ unbemerkt; (3) die Patientin wurde präoperativ über Möglichkeit einer Gefäßverletzung aufgeklärt - dem Operateur wurde rechtlich kein Vorwurf gemacht; (4) die diagnostischen und therapeutischen Maßnahmen waren korrekt (Gutachten).

Take-home message: Da eine Aortendissektion als Komplikation der laparoskopischen Magenbandimplantation bislang nicht beschrieben worden ist, soll dieser Fallbericht die Möglichkeit und das erfolgreiche Komplikationsmanagement darstellen. 


\section{P03}

Surgical elimination of the gastric digestion leads to an increased risk of food protein sensitization

S. Shakeri-Leidenmühler ${ }^{1}$, A. Lukschal ${ }^{2}$, C. Schultz ${ }^{2}$, A. Bohdjalian ${ }^{1}$, F. Langer1, T. Birsan ${ }^{1}$, S. C. Diesner², T. Kopp ${ }^{3}$, O. Scheiner ${ }^{2}$, E. Jensen-Jarolim², E. Untersmayr ${ }^{2}$, G. Prager ${ }^{1}$

${ }^{1}$ Department of Surgery, Medical University, Vienna, Austria; ${ }^{2}$ Department of Pathophysiology and Allergy Research, Center of Pathophysiology, Infectiology and Immunology, Medical University, Vienna, Austria; ${ }^{3}$ Department of Dermatology, Medical University, Vienna, Austria

Background. We have previously revealed that impairment of gastric digestion due to $\mathrm{pH}$ elevation represents a risk factor for food allergy induction. Therefore we aimed to analyze in a prospective study the effect of elimination of the gastric digestion by surgical intervention on the induction of food allergy.

Methods. Eleven patients undergoing Roux-en-Y gastric bypass surgery for morbid obesity were included in the study. They were compared to a control patient having undergone surgery for treatment of an incisional hernia. Before and 1, 3, 6, 9 and 12 months after the gastric bypass surgery blood was collected for specific IgE antibody analysis. Additionally, skin prick tests with 16 food and 18 aeroallergens were performed.

Results. An increase of positive skin reactivities indicating sensitizations towards the tested food compounds and towards the aeroallergens after the gastric bypass surgery were found in $72 \%$ of the patients. These results were confirmed by measurement of food-specific and aeroallergen-specific IgE antibody titers.

Conclusions. Surgical elimination of gastric digestion leads to a marked change in IgE reactivity pattern underlining the important gate keeping function of gastric digestion against food proteins.

\section{P05}

Kurz- und mittelfristige Resultate nach Gastric Sleeve Operation bei Patienten mit morbider Adipositas

\section{P. Neidenbach, T. Delko, T. Köstler, O. Schöb \\ Spital Limmattal, Schlieren, Switzerland}

Grundlagen. Die laparoskopische Gastric Sleeve (LGS) Operation wird zunehmend als alleinige Methode zur Gewichtsreduktion und Therapie adipositasassoziierte Komorbiditäten angewandt. Wir präsentieren unsere Resultate.
Methodik. Daten der Patienten, die von November 2008 bis Dezember 2010 einer LGS Operation erhielten, wurden ausgewertet. Geschlecht, Alter, Body Mass Index (BMI) und Komorbiditäten wurden erfasst. Bei Nachuntersuchungen nach 6 Wochen, 6 und 12 Monate wurden der Excess body weight loss (EBWL), postoperative Komplikationen und Veränderungen der Komorbiditäten ausgewertet.

Ergebnisse. 30 Patienten (20 Frauen, $66 \%$, Alter: 42,9 Jahre), wurden operiert. Der präoperative BMI lag bei den männlichen Patienten im Durchschnitt bei $50,1 \mathrm{~kg} / \mathrm{m}^{2}$, bei den weiblichen Patienten bei $49,7 \mathrm{~kg} / \mathrm{m}^{2}$. Als Komorbiditäten traten arterielle Hypertonie $(n=21,70 \%)$ und Typ II Diabetes mellitus $(n=13,43 \%)$ auf. Der BMI (EBWL) nach 6 Wochen war bei männlichen Patienten $41,7 \mathrm{~kg} / \mathrm{m}^{2}$ (26,1\%), bei weiblichen $44,2 \mathrm{~kg} / \mathrm{m}^{2}$ (20,1\%), nach 6 Monaten $35,6 \mathrm{~kg} / \mathrm{m}^{2} \quad(51,7 \%)$, respektive $39,4 \mathrm{~kg} / \mathrm{m}^{2} \quad(28,8 \%)$. Nach 12 Monaten $32,9 \mathrm{~kg} / \mathrm{m}^{2}$ (52,4\%), bzw. $36,2 \mathrm{~kg} / \mathrm{m}^{2}$ (50\%). Es kam postoperativ zu einer Insuffizienz der Staplernaht. Eine Refluxysmptomatik wurde bei 11 (36,6\%) Patienten beschrieben.

Schlussfolgerungen. Die Behandlung der morbiden Adipositas mittels LGS im kurz- bis mittelfristigen Verlauf ist eine mit wenigen Komplikationen verbundene Vorgehensweise, die einen guten Gewichtsverlust initiiert.

\section{Ausbildung}

\section{P06}

\section{Residents complication rate performance during surgical education}

\section{T. M. Koch ${ }^{1,2}$, H. J. Mischinger ${ }^{1,2}$, G. A. Bernhardt ${ }^{1,2}$ \\ ${ }^{1}$ LKH Voitsberg, Abteilung für Chirurgie, Voitsberg, Austria; \\ ${ }^{2}$ Medizinische Universität Graz/Universitätsklinik \\ für Chirurgie/Klinische Abteilung für Allgemeinchirurgie, Graz, Austria}

Background. The following work presents the advantages of a prospectively documentation of complications in a surgical department demonstrating the complication rate performance during surgical residency.

Methods. Over a five years period data sheets about post surgical complications classified according to Clavien's system were prospectively collected and entered in a electronically data base. The complication rates of two surgical residents were compared and analyzed relating to their years of residency.

Results. The over all resident's complication rate was $4.1 \%$ and did not differ when compared to the department's complication rate of $6.2 \%(p=0.507)$. There was no difference between the two residents $(4.6 \% v s$. $3.8 \% ; p=0.632$ ). The complication rate performance was 
similar showing an increase in first three years and a decrease of complications in the following time.

Conclusions. Complication rates start to increase when surgical residents start to perform more complex procedures or act more independently and therefore may cause more mistakes. Due to acquired experience complication rates start to decline afterwards. Furthermore young surgeons learn to assess their abilities and enlist assistance if necessary.

Educational guidance and assistance during surgery by experienced surgeons is essential to achieve low complication rates during residency and to allow a high quality surgical education.

\section{Gefäßchirurgie}

\section{P07}

\section{If you have a bleeding stop the bleeding -} Rupturiertes A. hepatica Aneurysma interventionell gelöst

\section{T. Niernberger ${ }^{1}$, T. Meissnitzer ${ }^{2}$, T. Ott ${ }^{1}$, M. Uggowitzer ${ }^{2}$, H. Rabl ${ }^{1}$ \\ ${ }^{1}$ Abteilung für Chirurgie, Leoben, Austria; ${ }^{2}$ Institut für medizinische Radiologie, Leoben, Austria}

Grundlagen. Nur 0,2\% aller arteriellen Aneurysmen betreffen den Truncus coeliacus und seine Äste. Davon ist wiederum in $20 \%$ der Fälle die Arteria hepatica betroffen.

Methodik und Ergebnisse. Wir berichten von einer 68 jährigen Patientin, welche nach auswärts mittels CTDiagnostik abgeklärtem rupturiertem Aneurysma des R. sinister der Arteria hepatica propria mit Einblutung in die freie Bauchhöhle zur definitiven Versorgung an unsere Abteilung zugewiesen wurde. Zum Zeitpunkt der stationären Aufnahme befand sich die Patientin in kreislaufstabilem Zustand, so daß man sich für ein interventionelles Vorgehen entschließen konnte. Der Verschluß des Aneurysmas erfolgte durch coiling. Die Entlassung erfolgte am 7. postinterventionellem Tag nach komplikationslosem Verlauf,mit vollstandiger Resorbtion der freien Flüssigkeit.

Schlussfolgerungen. Der interventionelle Approach ist bei gegebener Kreislaufstabilität sicherlich die für den Patient am wenigsten traumatisierende Therapieoption mit therapeutischem Erfolg unmittelbar am Ort des Geschehen. Gegenüber steht die Notfallslaparotomie mit Ligatur des Aneurysma bis hin zur Resektion mit Rekonstruktion mittels Interponat und einer Mortalität von 1$3 \%$. Nicht selten lassen sich bei Patienten mit Viszeralaterienaneurysmen solche auch zusätzlich auch an anderen Lokalisationen diagnostizieren. Es gilt letztendlich auch gezielt nach einer Aneurysmose zu fahnden.

\section{P08}

Central venous port complications: subclavian vein puncture vs. surgical cut-down to the cephalic vein

\section{B. Dauser, J. Stopfer, S. Ghaffari, F. Herbst}

Department of Surgery, St. John of God Hospital, Vienna, Austria

Background. Central venous ports (CVP) are commonly used in oncology but data comparing complications of different access techniques are rare. We present a retrospective study of two different insertion modalities from a single surgical department.

Methods. 352 CVP were implanted under local anaesthesia in the operation room between 2004 and 2009. In 250 (71\%) cases a non ultrasound-guided direct puncture (DP) of the subclavian vein was performed, $102(29 \%)$ times a cut-down (CD) to the cephalic vein was done. Primary success rate, procedure time and postoperative complications within 30 days were analyzed.

Results. Primary success rate for DP and CD was $96 \%$ and $89 \%$, respectively. Mean procedure time was slightly longer in the CD group (37.8 versus $32.6 \mathrm{~min}$; $p=0.083$ ). On routine postoperative chest radiography pneumothoraces were seen only following DP (6/250; $2.4 \%)$, once $(1 / 250 ; 0.4 \%)$ necessitating drainage. Explantation of CVP for early infection was done 5 times after DP and once after CD (2.0\% vs. $0.98 \%)$. In addition, operative revision due to malfunction of port $(n=5)$ or haematoma $(n=1)$ was necessary in the DP group only.

Conclusions. Surgical cut-down to the cephalic vein for CVP implantation is associated with a minimal risk for severe (early) complications.

\section{P09}

\section{Erfolgreiche Lyse einer akuten Mesenterialembolie}

\section{N. Fischbach, G. Fenzl, R. Klug}

Chirurgie, LK Horn, Horn, Austria

Wir berichten über die erfolgreiche Lyse einer akuten Mesenterialembolie bei einer 76-jährigen Patientin. Nach Aufnahme wird mittels Bildgebung ein akuter Mesenterialverschluß diagnostiziert. Aufgrund der Klinik, der kurzen Anamnese, sowie der raschen Diagnostik entscheiden wir uns im konkreten Fall für eine Lyse. Anhand dieses Falles wird das therapeutische Vorgehen und der Stellenwert der Lyse versus Embolektomie diskutiert. 


\section{P10}

\section{Rare Lokalisation eines seltenen Aneurysmas der A. hepatica im Abgangssegment der A. gastroduodenalis bei ebenfalls seltenem kombiniert-simultanen Auftreten eines Truncus hepatomesentericus}

\author{
P. Büschel ${ }^{1}$, M. Weber ${ }^{1}$, D. Hay ${ }^{1}$, M. Pech ${ }^{2}$, \\ Z. Halloul ${ }^{1}$, F. Meyer ${ }^{1}$ \\ ${ }^{1}$ Universitätsklinikum Magdeburg A.ö.R., Universitätsklinik \\ für Allgemein-, Viszeral- und Gefässchirurgie, Magdeburg, \\ Germany; ${ }^{2}$ Universitätsklinikum Magdeburg A.ö.R., \\ Universitätsklinik für Radiologie und Nuklearmedizin, \\ Magdeburg, Germany
}

Grundlagen. Viszerale arterielle Gefässaneurysmata sind selten, jedoch im Falle einer Ruptur hochgradig lebensbedrohlich. Es besteht eine hohe anatomische Variabilität der arteriellen Leberversorgung.

Methodik. Basierend auf einem klinischen Fall unter Bezugnahme auf diagnostisches Management und bisherigem mittelfristigen Verlauf soll die aussergewöhnliche Koinzidenz eines Aneurysmas der A. gastroduodenalis an der Abzweigung der A. hepatica bei gleichzeitigem Vorhandensein eines truncus hepatomesentericus dargestellt werden unter gleichzeitiger Aufarbeitung selektierter Literaturstellen.

Ergebnisse. Bei einer 39-jährigen Frau (BMI $24 \mathrm{~kg} / \mathrm{m}^{2}$ ) wurde zufällig ein Aneurysma der Arteria hepatica propria mit Übergriff auf die Arteria gastroduodenalis bei gleichzeitig vorliegender seltener Gefässversorgung der Leber durch einen Truncus hepatomesentericus diagnostiziert. Ein typischer Zusammenhang mit arterieller Verschlusskrankheit, mykotischer Embolisation, Trauma, Marfan-Syndrom, Klippel-Trenaunay-Syndrom oder Riesenzellarteriitis bestand nicht. Bei bisher moderatem Grössenwachstums auf nunmehr $18 \mathrm{~mm}$ innerhalb eines Jahres unterliegt die Patientin engmaschigen Kontrollen unter Zuhilfenahme von Duplexsonographiekontrollen als Methode der Wahl.

Schlussfolgerungen. Dies ist der erste in der Literatur beschriebene Fall, der (1) die Seltenheit eines Aneurysma der Arteria hepatica mit Übergriff auf die Arteria gastroduodenalis in Zusammenhang mit der (2) Variante eines Truncus hepatomesentericus - was die Häufigkeit dieser Koinzidenz potenziert \& unterstreicht die hohe Variabilitätsbreite der arteriellen Versorgung der Leber aus anatomischer Sicht, was zur Therapieplanung operativ-interventioneller Verfahren zu berücksichtigen ist.

\section{P11}

Pancreatitis-associated fluid collections: miniinvasive interventions

\section{S. Chooklin, O. Hranat}

Medical University, Lviv, Ukraine

Background. Experimental studies confirm that pancreatogenic ascites contributes to the emergence and progression of multiple organ dysfunction.

Methods. 141 patients with severe acute pancreatitis were studied. Parapancreatic fluid collections was detected in 120 patients $(85.1 \%)$, in the free abdominal cavity - in $83(58.9 \%)$. Simultaneous visualization was noted in 74 patients $(52.5 \%)$. Thus, pancreatogenic fluid was detected in 129 patients (91.5\%) with severe acute pancreatitis. Removal of inflammatory pancreatogenic fluid had 40 patients, of whom thirteen - laparoscopically.

Results. The presence of pancreatogenic fluid was significantly correlated with the presence of systemic complications. Evacuation of fluid improves the condition of patients, reduced the symptoms of intoxication. In this case, noted a gradual decrease in the blood of proinflammatory cytokines. Clearly improves the function of liver and kidney. It is important to note that only 6 (15\%) patients in this group were operated on for septic complications. The elimination of pancreatic ascites and accumulation of fluid can reduce the risk of complications $\left(\chi^{2}=11.50 ; p=0.0007\right)$.

Conclusions. Minimally invasive removal of pancreatogenic fluid collections should be used in treatment of patients with acute pancreatitis for the relief of organ dysfunction and prevention of septic complications.

\section{P12}

Die perkutane transhepatische Portanlage als letzte Chance bei multiplen Venenokklusionen bei einem Kleinkind

\section{Sachwitz ${ }^{1}$, S. Kroker ${ }^{1}$, H. Haß ${ }^{1}$, J. Ricke ${ }^{2}$, Z. Halloul ${ }^{3}$, F. Meyer ${ }^{3}$, H. Krause ${ }^{1}$}

${ }^{1}$ Universitätsklinikum Magdeburg, Klinik f. Allgemein-, Viszeral- u. Gefäßchirurgie,AB Kinderchirurgie, Magdeburg, Germany; ${ }^{2}$ Universitätsklinikum Magdeburg, Universitätsklinik für Radiologie und Nuklearmedizin, Magdeburg, Germany; ${ }^{3}$ Universitätsklinikum Magdeburg, Klinik f. Allgemein-, Viszeral- u. Gefäßchirurgie, Magdeburg, Germany

Grundlagen. Bei Kindern mit langzeitiger parenteraler Nutrition kommt es häufig zu Komplikationen der zentralvenösen Zugänge. Insbesondere wegen Infektionen, Katheter- und Venenokklusionen sind oft Katheter- bzw. Portanlagewechsel notwendig, wodurch 
sich die Optionen zentraler Gefäßzugänge früher oder später erschöpfen.

Methodik und Ergebnisse. Anhand einer ungewöhnlichen Fallkonstellation wird über die erfolgreiche alternative perkutane transhepatische Anlage eines vital erforderlichen Portkathetersystems in die Vena hepatica dextra bei einem Kleinkind mit ausgereizten konventionellen iv-Zugangsverhältnissen berichtet. Ein 5-jähriger Junge wurde 3 Tage post partum einer subtotalen Dünndarmresektion wegen eines ausgeprägten Volvulus' unterzogen, woraus ein Kurzdarmsyndrom resultierte. Die nachfolgend notwendige, supportive parenterale Ernährung erfolgte über diverse zentralvenöse Katheter, wobei aufgrund von Infektionen mehrere Katheterwechsel notwendig wurden. In der Folge waren die venösen Zugangsmöglichkeiten durch multiple Venenthrombosen in der Peripherie so stark eingeschränkt, dass als letzte Möglichkeit durch Interventionsradiologen eine perkutane transhepatische Portkatheteranlage erfolgreich durchgeführt wurde.

Schlussfolgerungen. Als Zugangsweg stellt die perkutane transhepatische Anlage eines Portkathetersystems eine geeignete und risikoarme Alternative dar, die vor allem im Kleinkindalter noch relativ selten genutzt wird. Mit dieser Technik steht ein sicherer alternativer iv-Zugangsweg unter Wahrung der minimal zumutbaren Invasivität mit zu anderen Gefäßzugängen vergleichbaren Komplikationen hinsichtlich Häufigkeit und Spektrum zur Verfügung, der im interdisziplinären Management einen erfahrenen Interventionsradiologen erfordert.

\section{P13}

Atypische Nutzung einer femorofemoralen AV-Prothese zur Erhaltung der Lebensqualität in Zusammenhang mit seltener Notwendigkeit täglicher Infusionen bei chronischem Asthma bronchiale über 2 Dekaden

\section{P. Büschel ${ }^{1}$, U. K. Fetzner ${ }^{2}$, Z. Halloul' ${ }^{1}$, F. Meyer ${ }^{1}$ \\ ${ }^{1}$ Universitätsklinikum Magdeburg A.ö.R., Universitätsklinik für Allgemein-, Viszeral- und Gefässchirurgie, Magdeburg, Germany; ${ }^{2}$ Universitätsklinikum Köln, Klinik für Allgemein-,Viszeral- und Tumorchirurgie, Köln, Germany}

Grundlagen. Die Notwendigkeit täglicher intravenöser Infusionen im Rahmen chronischer Erkrankungen zur Erhaltung der Lebensqualität ist in der heutigen Zeit als extrem selten anzusehen. Dennoch bestehen spezielle Indikationen die eine sichere Punktionsmöglichkeit auch im Notfall - ermöglichen. Anhand einer besonderen Falldarstellung mit 20 Jahre bestehendem femorofemoraler AV-Prothesenshunts soll diese seltene Konstellation der
1) Notwendigkeit von täglichen iv-Gaben,

2) Praktikabilität einer femoro-femoralen AV-Prothese und

3) Langjährige nahezu komplikationslose atypische Nutzung und Lokalisation dargestellt werden.

Methodik. Für eine 68-jährige Frau mit chronischem Asthma bronchiale besteht die Notwendigkeit der jahrzentelangen täglichen iv-Infusionstherapie mit Theophyllin. Nach Ausreizung der peripheren Zugangsmöglichkeiten erfolgten bei der Patientin die iv-Gaben via an den oberen Extremitäten angelegter AV-Fisteln - mit jedoch jeweils nur mittelfristigem Erfolg. Als ultima ration erhielt die Patientin vor nunmehr 20 Jahren einen femoro-femoralen AV-Prothesenshunt sowohl für die Routine, als auch für die Notfall- und ggf. Selbstpunktion aufgrund der günstigen Lage geeignet.

Ergebnisse. Im Rahmen der langjährigen Benutzung hatte sich bisher keine Infektions-, als auch Abstossungsreaktion der Prothese gezeigt. Lediglich einmalig musste bei akutem Verschluss eine Revision mit Thrombektomie erfolgen. Ein Steal-Phänomen konnte ebenfalls nicht beobachtet werden.

Schlussfolgerungen. Dies ist ein sehr seltener Fall einer atypischen Nutzung eines sicheren venösen Gefässzugangs über 2 Dekaden mit aussergewöhnlich komplikationsarmer täglicher Nutzung.

\section{Handchirurgie}

\section{P14}

\section{Die ambulante chirurgische Versorgung der Rhizarthrose}

\section{T. Radda, W. Freilinger, F. Landsiedl, C. Krasny}

orthopädisches Spital Speising 1. Abteilung, Wien, Austria

Wir bieten seit Herbst 2008 die Resektionsarthroplastik des Daumensattelgelenks als ambulanten Eingriff an.

Der Einweiser überprüft die Compliance und die Verfügbarkiet einer Begleitperson. Absolute Ausschlusskriterien sind: ASA Klassifikation schlechter als II, Myokardinfarkt in die letzten zwei Jahren, schlecht eingestellte arterielle Hypertonie, Dialysepatient, insulinpflichtiger Diabetiker, cerebraler Insult im letzten Jahr, Patient mit Ruhedyspnoe, Epilepsie und Myasthenia gravis. Eine Vollnarkose ist nur bei Patienten mit einem BMI unter 33 möglich.

Die Patienten werden etwa zwei Wochen vor dem Eingriff in einer präoperativen Ambulanz begutachtet und aufgeklärt. Postoperativ wird eine Unteramlonguette mit Daumeneinschluss angelegt und dem Patienten die Ödemprophylaxe gezeigt. Die Schmerztherapie besteht 
aus Naproxen und Paracetamol, sowie als Bedarfsmedikation Metamizol und bei sehr starken Schmerzen Hydromorphon. Der Patient erhält einen Entlassungsbrief sowie eine Notfalltelefonnummer.

Zwischen November 2008 und November 2010 wurden 15 Patienten (16 Operationen) zur tagesklinischen Resektionsarthroplastik eingewiesen. Nur ein Patient musste aufgrund von Schmerzen eine Nacht stationär bleiben. Keiner der Patienten rief die Notfalltelefonnummer an, suchte die Ambulanz vor der geplanten Kontrolle auf oder benötigte zur Schmerztherapie Hydromorphon. Die Anzahl der postoperativ benötigten Ergotherapieeinheiten ist nicht höher als die der stationär operierten Patienten.

Die Resektionsarthroplastik des Daumensattelegelenks ist bei Einhaltung bestimmter Standards und Richtlinien für uns eine ambulant gut durchführbare Operation.

\section{P15}

\section{Ambulante Handchirurgie bei Kindern und Jugendlichen?}

\section{W. Girsch}

Orthopädisches Spital Speising, Wien, Austria

Grundlagen. Der Zug der Zeit erfordert Eingriffe an der Hand zunehmend ambulant oder tageschirurgisch durchzuführen - gilt dies auch für Eingriffe an der Hand bei Kindern und Jugendlichen?

Methodik. Eingriffe -und nicht nur solche an der Hand - erfordern bei Kindern und Jugendlichen meist eine Allgemeinnarkose. Voruntersuchungen beim Kinderarzt und im Spital, sowie eine postoperative Überwachung sind erforderlich. HandchirurgischeEingriffe erfordern gerade bei Kindern meist eine kurzfristige Kontrolle des Verbandes am ersten postoperativen Tag. Die obligate Schmerztherapie kann oral oder rectal, muss eventuell auch parenteral erfolgen.

Ergebnisse. Nur wenige Eingriffe an der Hand - Pollex flexus, Ganglien - erlauben es, Kinder und Jugendliche sofort und bezüglich des Verbandes kontrollfrei, versorgt mit oralen Analgetika im Anschluss an den Eingriff zu entlassen.

Schlussfolgerungen. Wenige Argumente - va die Kostenproblematik - sprechen für die ambulante Handchirurgie bei Kindern und Jugendlichen, viele Argumente dagegen. Zeitdruck besteht niemals bei den jungen Patienten sondern wird wenn, von den Eltern verursacht. Häufige Besuche beim Arzt und im Spital prä- und postoperativ „stressen“ Kinder mehr als Erwachsene. Die postoperative Verordnung von Opiaten ist bei Kindern nicht möglich. Letztendlich muss die Behandlungsicherheit von ärztlicher Seite sichergestellt werden, da sie ansonsten von den Eltern gerichtlich eingeklagt wird.

\section{Kinderchirurgie}

\section{P16}

\section{Korrektur der proximalen Hypospadien: Ergebnisse und Komplikationen}

\section{A. Ardelean, C. Schimke, G. Schimpl}

Paracelsus Medizinische Privatuniversität, SALK, Universitätsklinik für Kinder- und Jugendchirurgie, Salzburg, Austria

Grundlagen. Ist bei proximalen Hypospadien die einzeitige Korrektur (EZK) oder die zweizeitige Korrektur (ZZK) anzustreben?

Methodik. 42 Patienten nach Korrektur proximaler Hypospadien wurden analysiert: 24 Patienten hatten eine penoscrotale- und 18 eine perineale Hypospadie. Bei allen 11 Patienten ohne Peniskrümmung wurde eine EZK durchgeführt. Bei 31 Patienten mit ausgeprägter Krümmung wurde die Urethralplatte durchtrennt und bei 13 eine EZK, bei 18 dieser Patienten die ZZK vorgenommen.

Ergebnisse. Komplikationen, die weitere chirurgische Eingriffe verlangten, wurden bei $46 \%(11 / 24)$ der Patienten nach EZK, und bei $17 \%$ (3/18) nach ZZK festgestellt. Die Komplikationsrate der EZK erreichte bei ausgeprägter Krümmung 61 \% (8/13), und bei den Fällen wo das Erhalten der Urethralplatte möglich war $27 \%$ (3/11). Die Nachuntersuchung bei 37 Patienten, zwischen 1 und 11 Jahren nach Ende der Behandlung, zeigte in $89 \%$ der Fälle ein gutes kosmetisches Ergebnis und in $11 \%$ ein befriedigendes Ergebnis. Die maximale Uroflowrate war sehr gut bei $5 \%$ (2/37), gut bei $54 \%(20 / 37)$ und schlecht bei $41 \%(15 / 37)$ der Patienten.

Schlussfolgerungen. Bei Patienten mit proximaler Hypospadie und ausgeprägter Peniskrümmung führt die zweizeitige Korrektur-Technik zu besseren kosmetischen und funktionellen Ergebnissen. Bei Patienten wo das Erhalten der Urethralplatte möglich ist, soll die einzeitige Korrektur angestrebt werden.

\section{Komplikationsmanagement}

\section{P17}

Case report einer spontanen intraabdominellen Blutung auf Grund einer eingeklemmten paraösophagealen Mischhernie

\section{K. Berger}

Abt. für Allgemeinchirurgie, St. Johann in Tirol, Austria 
Wir berichten über einen seltenen Fall einer spontanen, intraabdominellen Blutung auf Grund einer eingeklemmten paraösophagealen Mischhernie.

Eine 70 jährige Patientin wurde mit unspezifischen abdominellen, seit 3 Tagen bestehenden Oberbauchbeschwerden ohne Dysphagie aufgenommen. Bei der kreislaufstabilen Patientin zeigte das Aufnahmelabor einen $\mathrm{Hb}$ - Wert von 7,0, im AbdomenCT ein ausgeprägtes Hämoperitoneum mit partiellem upside down Magen, der anfänglich nicht als Blutungsursache bewertet wurde. In der diagnostischen Laparoskopie wurde ein Hämatom im Bereich der kleinen Kurvatur gefunden und die Blutungsquelle aus einem Seitenast der A. gastrica sinistra identifizert, wohl bedingt durch Zug oder mechanische Alteration kleinkurvaturseits im Rahmen der Inkarzeration. Es wurde konvertiert, der Magen in das Abdomen zurückverlagert, der Hiatus ohne Kunsstoffnetz verschlossen und die Hiatusplastik mit einer Nissenfundoplikatio gesichert. Der postoperative Verlauf (4 Tage ICU) war komplikationslos mit gut toleriertem Kostaufbau.

Die Symptompallette des partiellen Thoraxmagen reicht von symptomlos bis zum Magenvolvolus, wobei wir beim partiellen und totalen Thoraxmagen bei jüngeren Patienten die Indikation zur OP sehen. Eine Komplikation im Sinne eines Hämoperitoneum wurde bislang noch nicht dokumentiert.

\section{P18}

\section{Ausgedehnte iatrogene Tracheaverletzung - Fallbeschreibung}

\section{G. Prisadov, K. Welcker, H. Wallimann, A. Linder}

Klinikum Bremen-Ost, Bremen, Germany

Grundlagen. Die tracheobronchiale Verletzung bei Intubation ist ein seltenes Notfallereignis, aber mit einer hohen Sterblichkeit (bis zu 40\%). Wegen der Seltenheit gibt es keine evidenzbasierte Therapieleitlinien. Die Erfahrung hat gezeigt, dass kleinere Verletzungen (kleiner als $2 \mathrm{~cm}$ ) keiner Behandlung bedürfen. Bei zu beatmenden Patienten muss die Leckage durch eine entsprechende Tubuslage behandelt werden. Die größeren Verletzungen sollten möglich frühzeitig operativ versorgt werden.

Methodik und Ergebnisse. Wir berichten über einen 70 -jährigen polymorbiden Patienten mit einer ca. $10 \mathrm{~cm}$ langen Verletzung der distalen Tracheahinterwand nach Intubation bei respiratorischer Insuffizienz aufgrund einer Pneumonie mit ercheblicher Co-Morbidität. Klinisch hatte der Patient ein zunähmendes Gewebe- und Mediastinalemphysem. Bei der bronchoskopischen Beurteilung der Tracheaverletzung bildete sich ein Spannungspneumothorax rechts, der drainiert wurde. Die chirurgische Versorgung erfolgte durch eine primäre Naht der Trachea über eine anterolateralen Thorakotomie im 4.
ICR. Eine Langzeitbeatmung war erforderlich, und es erfolgte die Tracheostomie am 9 p.o. Tag. Der Niederdruck-Cuff lag im Bereich der Tracheanaht.

Schlussfolgerungen. Die frühzeitige operative Versorgung der ausgedehnten Tracheaverletzung ist der wichtigste Faktor für eine stabile Beatmungssituation. Die anterolaterale Thorakotomie ist für uns der Zugang der Wahl bei Verletzungen der distalen Trachea.

\section{P19}

\section{Lebensbedrohliche endobronchiale Blutung auf Grund einer abnormen Blutversorgung des linken Lungenunterlappens}

\section{W. Schreiner, O. Oster, M. Hanika, A. Zdrojek, H. Sirbu \\ Thoracic Surgery, University hospital, Erlangen, Germany}

Grundlagen. Die abnorme Blutversorgung eines basalen Segmentes des Unterlappens ist eine seltene Fehlbildung. In der Pryce-Klassifikation gehört die abnorme Blutversorgung von normalen Lungenabschnitten zum Typ 1.

Methodik. Wir berichten über einen 25jährigen Patienten, der ein akutes Lungenversagen auf dem Boden einer nicht beherrschbaren endobronchialen Blutung aus dem linken Unterlappen entwickelte.

Zwei abnorme Lungenarterien mit Ursprung aus dem Aortenbogen wurden primär gekoilt. Aufgrund der persistierten Blutung erfolgte Bronchusblockade des linken Unterlappenbronchus. Die Oxygenierung und $\mathrm{CO} 2$-Eliminierung unter ECMO waren infolge der ausgedehnten Lungeneinblutung nicht adäquat. Bei zunehmender Kreislauf-Instabilität durch ein Rechtherzversagen (PAP $45 \mathrm{mmHg}+\mathrm{ZVD}$ ) wurde eine Notfall-Thorakotomie durchgeführt. Drei weitere abnorme Arterien aus der Aorta descendens wurden ligiert. Durch eine Bronchiotomie des linken Hauptbronchus wurde das endobronchiale Hämatom evakuiert. Unter Verneblung mit Prostacyclin normalisierte sich der pulmonalarterielle Druck. Die postoperative Kontrolle nach einem Jahr zeigte einen normal sozial integrierten Patienten.

Ergebnisse und Schlussfolgerungen. Die Histologie konnte keine Gefäßmalformationen oder abnormes Lungengewebe nachweisen. Die Präparation zeigte einen regulären venösen Abfluss. Somit ist diese Veränderungen dem Typ I der Pryce-Klassifikation zuzuordnen. Im unseren Fall zeigten sich fünf abnorme Arterien, die die Fulminanz und Persistenz der Blutung erklären. Diese seltene Fehlbildung führte zu einer lebendbedrohlichen Situation, die mit maximaler intensivmedizinischer Therapie und durch eine Notfall-Thorakotomie zu beherrschen war. 


\section{Krebstherapie}

\section{P20}

\section{Der klinische Stellenwert der Photodynamen Therapie (PDT) in der Thoraxchirurgie}

\section{J. Lindenmann, V. Matzi, N. Neuboeck, C. Porubsky, U. C. Anegg, A. Maier, F. M. Smolle-Juettner}

Klinische Abteilung für Thorax -u. Hyperbare Chirurgie, Univ.Klinik für Chirurgie, Medizinische Universität Graz, Graz, Austria

Grundlagen. Die Prognose von Patienten mit malignem Pleuramesotheliom, inoperablem fortgeschrittenen Ösophagus-, oder Bronchuskarzinom, ist nach wie vor sehr trist. Die Photodyname Therapie (PDT) stellt eine sehr wirkungsvolle Option in der Palliativtherapie dieser Malignome dar.

Methodik. Die Funktionsweise der PDT beruht auf der Illuminierung von Malignomen nach vorangegangener intravenöser Photosensibilisierung und konsekutiver selektiver Tumorzellnekrose. Im Falle des Ösophaguskarzinoms als auch der malignen Bronchusstenose wird die PDT endoskopisch durchgeführt, beim malignen Pleuramesotheliom geschieht dies intra-operativ im Anschluss an die Dekortikation.

Ergebnisse. Mit Hilfe der PDT wird eine signifikante Reduktion der endoluminalen bzw. intrakavitären Tumormasse, verbunden mit deutlich verbesserter Lebensqualität und verlängertem rezidiv-freiem Intervall, erreicht. Außerdem kann der Patient dadurch für weitere palliative Therapiemaßnahmen konditioniert werden.

Schlussfolgerungen. Die PDT stellt eine sichere, gut durchführbare und äußerst wirksame Option in der endoskopischen Palliativtherapie des fortgeschrittenen Ösophagus -, als auch des Bronchuskarzinoms dar. Überdies kann die PDT als supportive Maßnahme in der chirurgischen Therapie des malignen Pleuramesothelioms eingesetzt werden.

\section{P21}

Intraoperative Radiotherapie (IORT) eine alternative Behandlungsform für das funktionell nicht operable nicht kleinzellige Bronchuskarzinom (NSCLC)

N. Neuböck ${ }^{1}$, J. Lindenmann ${ }^{1}$, V. Matzi ${ }^{1}$, G. Jakse ${ }^{2}$, A. Oechs ${ }^{2}$, K. Kapp ${ }^{2}$, A. Maier ${ }^{1}$, F. Smolle-Jüttner ${ }^{1}$

${ }^{1}$ Department für Thorax-und Hyperbare Chirurgie, Graz, Austria; ${ }^{2}$ Department of Therapeutic Radiology and Oncology, Graz, Austria
Grundlagen. Lungenkrebs bleibt eine der häufigsten krebsassoziierten Todesursachen. Bei Limited Disease Non Small Cell Lung Cancer (NSCLC) ist die chirurgische Resektion Therapie der Wahl. Schlechter Allgemeinzustand und funktionelle oder anatomische Inoperabilität erfordern alternative Therapieformen.

Methodik. Eine 1987 initiierte Studie, welche die intraoperative Radiationstherapie (IORT) kombiniert mit externer Bestrahlung (EBI) als therapeutische Alternative für Patienten untersucht, deren reduzierte Gesamtsituation eine Thoracotomie jedoch keine Lobektomie erlaubt, umfasst bislang 52 Patienten - 14 weibliche (26,9\%), 38 männliche $(73,07 \%)$ zwischen 49 und 80 Jahren (Median: 65). Die IORT erfolgte jeweils via posterio-laterale Thoracotomie. 50 Patienten wurden 4 Wochen nach der IORT extern bestrahlt.

Ergebnisse. 50 Patienten beendeten die EBI, die statistische Überlebensrate betrug 71,2\%, 55,8 \%, 34,6\%, die erkrankungsspezifische $76 \%, 63,2 \%$ und $49,5 \%$ jeweils nach 12, 24 und 36 Monaten.

Schlussfolgerungen. Die Kombination von IORT mit EBI ist eine effiziente, brauchbare und gut verträgliche Therapiemöglichkeit für Patienten mit NSCLC mit Untauglichkeit für eine Lungenresektion.

\section{P22}

\section{Unterschiede zwischen Karzinomen} des linken bzw. rechten Kolons in Bezug auf Epidemiologie, perioperativen Verlauf, Histologie und Überleben: Analyse von 17641 Patienten

\author{
F. Benedix ${ }^{1}$, F. Meyer ${ }^{1}$, R. Kube ${ }^{2}$, H. Lippert ${ }^{1}$ \\ ${ }^{1}$ Otto-von-Guericke Universität, Magdeburg, Germany; \\ ${ }^{2}$ Carl Thiem Klinikum, Cottbus, Germany
}

Es gibt Hinweise darauf, dass es sich beim Kolonkarzinom (CC) in Abhängigkeit von der anatomischen Lage rechts- (RCC) bzw. linksseitige Kolonkarzinome (LCC) möglicherweise um verschiedene Tumorentitäten handelt. Ziel der vorliegenden Studie ist die Klärung, inwieweit sich diese Unterschiede epidemiologisch, klinisch, histologisch, im perioperativen sowie im onkologischen Langzeit-Outcome bemerkbar machen. In einem 3-Jahres-Zeitraum wurden alle konsekutiven Patienten der deutschen multizentrischen Beobachtungsstudie mit einem Kolonkarzinom hinsichtlich demografischer Daten und histologischer Parameter analysiert.

Für die Subgruppe der kurativ Resezierten erfolgte die zusätzliche Analyse folgender Parameter: perioperativer Verlauf, TNM- und UICC Stadium, Überleben. Es wurden insgesamt 17.641 Patienten erfasst, $71.1 \%$ davon wurden kurativ reseziert. RCC fanden sich häufiger bei Frauen sowie im höheren Lebensalter. Die histopathologischen Befunde waren bei den RCC häufiger lokal fortgeschritten 
und niedriger differenziert. Darüber hinaus gab es einen höheren Anteil muzinöser, undifferenzierter und siegelringzelliger Karzinome. Die Gesamtrate synchroner Metastasen unterschied sich nicht jedoch die Art der Metastasierung. Patienten mit LCC hatten ein besseres onkologisches Langzeit-Outcome.

Für Karzinome des linken bzw. rechten Kolons lassen sich signifikante Unterschiede hinsichtlich verschiedener Parameter nachweisen. Als Ursache hierfür kommen die zahlreichen genetischen Unterschiede in Frage. Die Unterschiede zwischen RCC und LCCechts- bzw. linksseitigen Karzinomen sollten im Rahmen der Karzinomprävention sowie bei klinischen Studien berücksichtigt werden.

\section{P23}

Seltener Inflammatorischer Pseudotumor im mesenterialen Fettgewebe des Dünndarms: Fallbericht eines 39-jährigen Patienten

\author{
M. Petersen ${ }^{1}$, D. Küster ${ }^{2}$, A. Roessner ${ }^{2}$, K. Schütte ${ }^{3}$, \\ H. Lippert ${ }^{1}$, F. Meyer ${ }^{1}$ \\ ${ }^{1}$ Klinik für Allgemein-, Viszeral- \& Gefäßchirurgie, \\ Magdeburg, Germany; ${ }^{2}$ Institut für Pathologie, Magdeburg, \\ Germany; ${ }^{3}$ Klinik für Gastroenterologie, Hepatologie \\ und Infektiologie, Magdeburg, Germany
}

Grundlagen. Der selten vorkommende inflammatorische myofibroblastäre Pseudotumor (IMT) ist eine relevante Differenzialdiagnose in Bezug auf Häufigkeit, Abgrenzbarkeit und adäquates Management in Diagnostik und Therapie von Darm-assoziierten Tumoren.

Methodik und Ergebnisse. Anhand eines außergewöhnlichen Fallberichts wird der IMT zu Auftreten, Diagnosefindung, Therapiemaßnahmen und „Outcome“ vor dem Hintergrund der relevanten Literaturangaben näher charakterisiert. Bei einem 39-jährigen Mann wurde 12 Jahre nach reseziertem rechts-pulmonalen IMT ein multifokales Tumorrezidiv im rechten Thorax und eine singuläre Zweitmanifestation im Bereich des jejunalen Mesenteriums diagnostiziert. Es erfolgte die Resektion des im mesenterialen Fettgewebe lokalisierten inflammatorischen Konglomerattumors. Eine operative Reintervention bezüglich der pulmonalen/thorakalen Rundherde wurde aufgrund der V.-cava-superior-nahen Lokalisation, mediastinaler Infiltration sowie der Anzahl thoraxchirurgisch nicht favorisiert. Eine empfohlene immunsuppressive Kombinationstherapie lehnte der Patient ab, stimmte jedoch einer passager-systemischen Steroidtherapie zu. Den empfohlenen CT-gestützten Verlaufskontrollen kam der Patient nur einmalig 12 Monate postoperativ nach (mäßiger lokaler Progress), so dass erneut eine Chemotherapie empfohlen, jedoch vom Patienten wiederum abgelehnt wurde (alternativ: Periodische Steroidgabe mit schrittweiser Dosisreduktion).
Schlussfolgerungen. Es liegt hier einer der selten berichteten Fälle eines multifokalen IMT-Rezidives im rechten Thorax 12 Jahre nach primärer Resektion mit seltener Zweitmanifestation/(DD) Metastase ungewöhnlicher, da sonst bevorzugt infantiler Lokalisation im Bereich des jejunalen Mesenteriums vor.

\section{P24}

\section{Eine Monozytenpopulation als möglicher Resistenzfaktor in der anti-VEGF Tumortherapie}

\section{Schauer ${ }^{1}$, P. Starlinger ${ }^{1}$, C. Reiter ${ }^{1}$, T. Maier ${ }^{1}$, L. Alidzanovic ${ }^{1}$, C. Nemeth ${ }^{1}$, B. Herberger ${ }^{1}$, L. Pop ${ }^{1}$, B. Grünberger ${ }^{2}$, T. Grünberger ${ }^{1}$, C. Brostjan ${ }^{1}$}

${ }^{1}$ Medizinische Universität Wien, Allgemeinchirurgie, Wien, Austria; ${ }^{2}$ Abteilung für Innere Medizin, Krankenhaus der Barmherzigen Brüder, Wien, Austria

Grundlagen. Eine Vielzahl an Patienten mit metastasiertem Kolorektalkarzinom zeigt im Rahmen der Behandlung einen Wirkungsverlust unter anti-VEGF Therapie mit Bevacizumab. Die Resistenzmechanismen sind jedoch weitgehend unbekannt, daher sind prädiktive Marker von großem Interesse. Wir haben in diesem Zusammenhang die Population der TIE2exprimierenden Monozyten (TEMs) untersucht. TEMs sind charakterisiert durch die Expression des Angiopoietin Rezeptors TIE2 und haben im Tiermodell angiogenetische Wirkung gezeigt, d.h. das Tumorwachstum durch parakrine Stimulation der Gefäßbildung gefördert.

Methodik. TEMs wurden im Blut von 30 Patienten mit kolorektalen Lebermetastasen, welche eine Leberresektion erhielten, vor und nach neoadjuvanter sowie adjuvanter Chemotherapie mit Bevacizumab über den Nachweis von CD14, CD16 und TIE2 Expression mittels Durchflusszytometrie bestimmt.

Ergebnisse. TEMs sind nach Bevacizumab Therapie signifikant erhöht. Der Anstieg ist neoadjuvant und adjuvant nachweisbar. Hingegen ist keine TEM Induktion bei Chemotherapie ohne Bevacizumab Gabe zu verzeichnen. Es besteht kein genereller Anstieg der Monozytenzahl bei diesen Patienten.

Schlussfolgerungen. Die Bevacizumab Therapie führt zu einer erhöhten Anzahl von zirkulierenden TEMs, die aufgrund ihres stark pro-angiogenetischen Effekts der systemischen VEGF-Blockade entgegenwirken könnten. Die Auswirkungen der Bevacizumab Therapie auf die Funktion der TEMs wird derzeit genauer analysiert, um ihre mögliche Rolle als Resistenzmechanismus in der anti-VEGF Tumortherapie evaluieren zu können. 


\section{P25}

\section{A non-pediatric case of lipofibromatosis}

\section{J. Friesenbichler ${ }^{1}$, A. Leithner ${ }^{1}$, A. Beham ${ }^{2}$, R. Windhager ${ }^{3}$}

${ }^{1}$ Department of Orthopedic Surgery, Graz, Austria; ${ }^{2}$ Institute of Pathology, Graz, Austria; ${ }^{3}$ Department of Orthopedic Surgery, Vienna, Austria

Background. Lipofibromatosis is an extremely seldom not malignant pediatric soft tissue tumor, characterized by slow and painless growth, with a predilection for distal extremities, a poor margination and a high local recurrence rate. The histology consists of abundant adipose tissue traversed by bundles of spindled fibroblastic cells.

Methods and results. We present the case of a 25year-old female patient with a retroclavicular growing tumor. The expansion caused recurrent paresthesia in the left hand. The MR imaging of the affected cervico-thoracal area showed a $4.6 \times 4.3 \times 6.4$ centimeters measuring encapsulated lesion, reaching up to the supraspinatus muscle. An incisional biopsy was performed for diagnostic reasons, leading to the diagnosis of lipofibromatosis. As a consequence the tumor was resected marginally. During a follow-up period of 48 months no local recurrence was detected.

Conclusions. In past lipofibromatosis was interpreted as infantile or juvenile fibromatosis, fibrous hamartoma or fibrosing lipoblastoma which may explain the rarity of this entity. To the best of our knowledge this is the first report of a non-pediatric case of lipofibromatosis. In conclusion the entity of lipofibromatosis should be kept in mind as a potential differential diagnosis for fibrofatty tumors in children as well as young adults.

\section{P26}

Influence of costunolide and dehydrocostus lactone on cell cycle and $A B C B 1 / M D R 1$ expression of human soft tissue sarcoma cell lines

\section{B. Lohberger ${ }^{1}$, B. Rinner ${ }^{2}$, A. Novak ${ }^{2}$, N. Stuendl ${ }^{1}$, N. Kretschmer ${ }^{3}$, R. Bauer ${ }^{3}$, A. Leithner ${ }^{1}$ \\ ${ }^{1}$ Department of Orthopaedic Surgery, Medical University of Graz, Graz, Austria; ${ }^{2}$ Center for Medical Research, Medical University of Graz, Graz, Austria; ${ }^{3}$ Institute of Pharmaceutical Sciences, Department of Pharmacognosy, Karl-Franzens- University, Graz, Austria}

In this study, we investigated the anti-tumor effects of the sesquiterpene lactones costunolide and dehydrocostus lactone, isolated from Saussurea lappa (SL), on human soft tissue sarcoma (STS) cell lines of various origins. Both compounds inhibited cell proliferation of all STS cell lines in a dose- and time-dependent manner at concentrations ranging from $2.5 \mu \mathrm{g} / \mathrm{ml}$ to $7.5 \mu \mathrm{g} / \mathrm{ml}$ and incubation periods from 24 to $72 \mathrm{~h}$. Costunolide treated cells showed no changes in cell cycle, no depolarisation of the mitochondrial membrane potential or expression of cleaved caspase-3 after 24, 48, and $72 \mathrm{~h}$. Dehydrocostus lactone however caused a significant reduction of the Gl phase and an increase in $\mathrm{S}$ and $\mathrm{G} 2 / \mathrm{M}$ phases as well as expression of cleaved caspase-3. In addition, the influence of costunolide and dehydrocostus lactone on the expression of ABCG2/BCRP1, ABCA2 and ABCB1/MDR1 proteins were examined using real-time RT-PCR. Dehydrocostus lactone significantly downregulated the expression of $\mathrm{ABCB1} / \mathrm{MDR} 1$ in the liposarcoma and synovial sarcoma cell lines. In summary, our data demonstrate that especially dehydrocostus lactone affected the cell cycle and ABCB1/MDR1 expression. Furthermore, it leads to expression of cleaved caspase- 3 and cleaved PARP when used a concentration that is twice the IC50.

\section{P27}

\section{The role of p38 MAPK pathway in human colon cancer cell resistance to 5 -fluorouracil}

\author{
Y. Chan, A. Miah, A. Bartnik, B. Fuller, S. Yang, \\ M. Winslet
}

Division of Surgery and Interventional Science, Royal Free Hospital and University College London Medical School, London, UK

Background. Although 5-fluorouracil (5-FU) has been widely used in the treatment of patients with advanced colon cancer, its therapeutic effect is often constrained by various chemoresistance mechanisms. Recent evidence suggests that the stress-activated p38 mitogen-activated protein kinase (MAPK) pathway is involved in oncogenic behaviour of colon cancer cells and may therefore be used as a target to potentiate 5-FU effect.

Methods. 5-FU sensitive SW620 and 5-FU resistant HT29 cells were treated with p38 MAPK inhibitor (SB203580) as a single agent or in combination with 5FU and then evaluated using CellTiter Blue Cell Viability Assay, inverted microscopy, immunofluorescence and Western blot.

Results. We found that inhibition of p38 MAPK alone tended to increase SW620 cell growth but slightly reduced HT29 cell survival with a significant induction of autophagy. In combination therapy, p38 MAPK inhibitor suppressed the inhibitory action of 5-FU in SW620 cells by $17.6 \%$ ( $p=0.029$ ) but was able to enhance the effect of 5FU in HT29 cells with an additional gain in inhibition of $19.7 \%$ ( $p 0.005)$.

Conclusions. Our results suggest that p38 MAPK is involved in colon cancer cell homeostasis following 5-FU treatment, and manipulation of the p38 MAPK pathway can augment colon cancer cell sensitivity to 5-FU treatment. 


\section{P28}

\section{Preoperative capecitabine, radiation and bevacizumab in rectal cancer}

\author{
M. Omejc ${ }^{1}$, V. Velenik ${ }^{2}$, J. Ocvirk², M. Music ${ }^{2}$, \\ M. Bracko ${ }^{2}$ \\ ${ }^{1}$ Department of Abdominal Surgery, Ljubljana, Slovenia; \\ ${ }^{2}$ Institut of Oncology, Ljubljana, Slovenia
}

Background. Role of bevacizumab to capecitabinebased neoadjuvant radiochemotherapy to increase the pathological remission rate (TRG) of locally advanced rectal cancer.

Methods. Sixty-one patients with MRI-confirmed stage II/III rectal cancer were treated with an infusion of Bev 2 weeks prior to neoadjuvant CRT, followed by Bev $5 \mathrm{mg} / \mathrm{m}^{2}$ on week 3, 5, 7 and capecitabine $825 \mathrm{mg} / \mathrm{m}^{2}$ bid including weekends during RT. RT was administered at $50.4 \mathrm{~Gy}$, starting on week 3 . TME was scheduled 6-8 weeks after completion of CRT.

Results. The median tumor distance from anal verge was $6(0-11) \mathrm{cm} .3$ patients presented with stage T2 (4.9\%), 53 T3 (86.9\%), 5 T4 (8.2\%). Lymph node involvement was detected in 49 patients $(80.3 \%)$. In 28 pts (45.9\%) tumor invaded the mesorectal fascia. Radical resection was achieved in 57 pts (95\%), 42 pts (70\%) had sphincter preserving surgery. TRG 4 (pCR) was recorded in 8 pts (13.3\%), TRG 3 in 9 pts (15\%). 38 pts (62.3\%) developed perioperative complications: delayed wound healing $(n=18,30 \%)$, infection/abscess $(n=12,20 \%)$, anastomotic leak $(n=7,11.7 \%)$.

Conclusions. The high rate of radical resections suggests its potential effect on tumor downstaging. However, it is associated with considerable toxicity and no clinically relevant increase in TRG.

\section{P29}

Synchroner Whipple und Teilnephrektomie bei Doppelkarzinom ohne präoperativ konklusive Pankreasdiagnostik - ist der Whipple auf Verdacht gerechtfertigt?

\author{
A. Hauer ${ }^{1}$, R. Klug ${ }^{1}$, H. Reiter ${ }^{2}$ \\ ${ }^{1}$ Chirurgie, LK Horn, Horn, Austria; ${ }^{2}$ Urologie, \\ LK Waidhofen/Thaya, Waidhofen/Thaya, Austria
}

Die 58jährige Patientin unterzog sich einer ERCP mit EPT und Steinextraktion bei symptomatischer Choledocholithiasis. Dabei wurde ein temporärer Stent wegen eines extraluminalen KM Depots gesetzt. Binnen einer Woche nach ERCP führten wir die laparaskopische Cholezystektomie durch und entfernten den Stent nach 6 Wochen. Danach kommt es erneut zum Auftreten einer
Cholestase mit deutlichem Bilirubinanstieg. Eine neuerliche ERCP mit Bürstenabstrich und Probeentnahmen wird durchgeführt und in gleicher Sitzung eine Gallengangsdrainage eingebracht. Die Histologie ergibt eine sklerosierende Papillitis. Nach neuerlicher Stententfernung entwickelt sich wiederum eine Cholestase. Die weitere Bildgebung kann einen kleinen tumorösen Prozess im Papillenbereich nicht sicher ausschließen. Zusätzlich besteht radiologisch der Verdacht eines Hypernephroms rechts, sodass wir mit der Patientin die operative Exploration mit ggf. onkologischer Resektion am Pankreaskopf und zusätzlich die Nierenteilresektion besprechen. Intraoperativ läßt sich der Verdacht auf einen Pankreaskopftumor nicht ausräumen, sodass wir eine Resektion nach Whipple durchführen. In gleicher Sitzung wird seitens der Urologie die Teilnephrektomie durchgeführt. Das histologische Präparat zeigt ein Pankreaskopfkarzinom pT2pN0, R0, G2 und ein klarzelliges Nierenzellkarzinom pT1pNx, G1.

Aufgrund der äußerst schlechten Prognose des Pankreaskopfkarzinoms ist die Radikaloperation nach Whipple auch ohne prä- und intraoperative Diagnosesicherung bei nicht ausräumbarem Verdacht gerechtfertigt. Voraussetzung ist eine institutionell hohe Ergebnisqualität.

\section{P30}

\section{Prognostic value of commonly measured laboratory parameters in patients with biliary malignancies}

\author{
E. Eichmann ${ }^{1}$, F. Eisner ${ }^{1}$, T. Stojakovic ${ }^{2}$, T. Kiesslich ${ }^{3}$, \\ C. Lackner ${ }^{4}$, T. Bauernhofer ${ }^{1}$, H. Mischinger ${ }^{5}$, \\ H. Samonigg ${ }^{1}$, M. Pichler ${ }^{1}$ \\ ${ }^{1}$ Division of Oncology, Medical University of Graz, Graz, \\ Austria; ${ }^{2}$ Clinic for Laboratory Medicine, Medical University \\ of Graz, graz, Austria; ${ }^{3}$ Department of Internal Medicine, \\ Paracelsus Medical University Salzburg, Salzburg, Austria; \\ ${ }^{4}$ Institute of Pathology, Graz, Austria; ${ }^{5}$ Department \\ of Visceral Surgery, Medical University of Graz, Graz, Austria
}

Background. Biliary tract cancer is an aggressive disease with a high cancer-specific mortality despite curative resection. The aim of the present study was to identify whether commonly measured laboratory values can predict the cancer-specific survival after resection.

Methods. A retrospective study of ninety-three patients who underwent radical resection of biliary malignancies was performed. Clinicopathological parameters and commonly determined laboratory parameters including bilirubin, liver enzymes, LDH, CA19-9, CEA and Creactive protein were recorded. Kaplan-Meier survival curves were created and compared using log-rank analysis. Multivariate analysis was undertaken using Cox proportional hazards regression.

Results. There were 52 (56\%) extrahepatic cholangiocarcinomas and 41 (44\%) intrahepatic cholangiocarcino- 
mas included in this study. Univariate survival analysis identified a pre-operative elevated bilirubin, elevated Creactive protein and CA19-9 levels $>1000 \mathrm{U} / \mathrm{ml}$ as negative prognosticators $(p<0.05)$ of cancer-specific survival. However, using multivariate analysis only increased CA19-9 levels were confirmed as an independent negative prognostic factor $(p<0.05)$.

Conclusions. Elevated pre-operative CA 19-9 levels were found to be an independent predictor of poor cancer-specific survival after attempted curative resection for biliary tract cancer. Pre-operative measurement of CA199 levels in patients with biliary tract cancer should be routinely performed and might facilitate individual risk management.

\section{P31}

Multipel rezidivierendes, jedoch jeweils mit kurativer Intention reseziertes retroperitoneales Liposarkom - 19 Jahre nach Erstdiagnose bei stabiler pathohistologischer Morphologie jedoch wechselndem Grading (außergewöhnlicher Fallbericht)

\section{P. Büschel ${ }^{1}$, H. U. Schulz ${ }^{1}$, T. Kalinski ${ }^{2}$, H. Lippert ${ }^{1}$, F. Meyer ${ }^{1}$ \\ ${ }^{1}$ Universitätsklinikum Magdeburg A.ö.R., Universitätsklinik für Allgemein-, Viszeral- und Gefässchirurgie, Magdeburg, Germany; ${ }^{2}$ Universitätsklinikum Magdeburg A.ö.R., Zentrum für Pathologie und Rechtsmedizin, Institut für Pathologie, Magdeburg, Germany}

Grundlagen. Goldstandard der kurativen Therapie des Liposarkoms ist die R0-Resektion. Demonstriert wird der außergewöhnliche Casus eines retroperitonealen Liposarkoms, welches trotz initialer R0-Resektion vor 19 Jahren mehrfach rezidivierte. Aufarbeitung eines seltenen langfristig-klinischen Verlaufes anhand der diagnostischen sowie pathomorphologischen Tumoreigenschaften bei jeweils nur mittelfristig tumorfreien „Outcome“.

Methodik und Ergebnisse. Ein mittlerweile 70-jähriger Mann wurde inital mit einem $23 \times 29 \times 15 \mathrm{~cm}$ großen retroperitonealen Liposarkom diagnostiziert primär R0-reseziert. Im Rahmen der Tumornachsorge wurden insgesamt 18 Tumorrezidivmanifestationen innerhalb von 10 Episoden (durchschnittliche tumorfreie Zeitdauer: 20,04 Monate) diagnostiziert. Bei Folgeeingriffen konnte in kurativer Intention überwiegend R0-reseziert bzw. im Folgeeingriff nachreseziert werden. Die durchschnittliche Präparategröße betrug $8,065 \mathrm{~cm}$. Die histopathologische Aufarbeitung der Tumorrezidive bestätigte jeweils das Liposarkom mit variierenden Gradings. Durch das teilweise verdrängende Tumorwachstum wurden im Krankheit- sverlauf auch multiviszerale Resektionen notwendig, wobei derzeit eine persistierende enterokutane Fistel vorliegt.

Schlussfolgerungen. Die kurative Therapieintention mittels chirurgischer Resektion ist auch beim Liposarkomrezidiv eine aussichtsreiche Therapieoption, da bei jeweils erfolgreichem R0-Resektionsstatus auch bei multipel rekurrentem Liposarkom ein mittel- bis langfristiges, tumortherapeutisch beherrschbares „Outcome“ mit signifikanten tumorfreien Perioden erreicht werden kann. Dies wird eindrucksvoll und erstmals in einer deratigen Eingriffs- \& Präparateserie im vorgestellten spektakulären Fall demonstriert. Bei dieser Sarkomentität erscheint das Tumorrezidiv-assoziierte, prognostisch eher günstigere Grading diesen Langzeitverlauf $\mathrm{zu}$ begünstigen. Engmaschige Tumornachsorge mit bildgebenden Verfahren ist hierfür unerlässlich.

\section{P32}

\section{Sigmaresektionen von 2004 bis 2010 im BKH Kufstein}

\section{T. K. Grißmann, T. Ratschiller, B. Spechtenhauser, B. Furtmüller}

Division of general surgery, general hospital Kufstein, Kufstein, Austria

Grundlagen. Laparoskopische Operationen finden zunehmend in der kolorektalen Chirurgie Anwendung, auch in der Tumorchirurgie. Anhand einer retrospektiven Datenbank wurde die Entwicklung der Chirurgie am Sigma an unserer Abteilung kritisch aufgearbeitet.

Methodik. Im Beobachtungszeitraum von 01/2004 bis $12 / 2010$ wurden an unserer Abteilung 462 Sigma/Rektumresektionen durchgeführt. (217 Frauen, 245 Männer), mittlere Alter 64 (26-93), mittlerer BMI von 26,2 (25,7 bei Frauen, 26,5 bei Männern), ASA 2,37 (1-4). Indikation zur Operation waren 292 benigne und 170 maligne Erkrankungen, 12,7\% waren Akuteingriffe.

Ergebnisse. 388 der Operationen erfolgten primär laparoskopisch (83\%), in 3,2\% Operationen wurde zur offenen Methode konvertiert, 74 Eingriffe erfolgten primär per Laparotomiam. Die durchschnittliche Operationszeit betrug 149 Minuten, die mittlere Verweildauer lag bei 14 Tagen (2-73). Die häufigsten Komplikationen in der Frühphase war die Anastomoseninsuffizienz $(n=27)$ und p.s.Wundheilung, im weiteren Verlauf Hernien und andere.

Schlussfolgerungen. Die Anzahl der laparoskopische Sigmaresektionen nahm im beobachteten Zeitraum deutlich zu. Sie sind sowohl bei benignen als auch maligen Erkrankungen sicher durchzuführen. Mit einer Komplikationsrate von $6.9 \%$ liegen wir im guten internationalen Vergleich. 


\section{P33}

\section{Routinemäßige MR-Mammographie bei stanzbioptisch gesichertem Mammakarzinom/DCIS-operative Konsequenz?}

\section{H. Sheu, M. Miesbauer, D. Heck, R. Helfgott, S. Pöstlberger, M. Aufschnaiter}

Krankenhaus der Barmherzigen Schwestern in Linz, Linz, Austria

Grundlagen. MR-Mg werden nicht routinemäßig bei der präoperativen Abklärung eingesetzt. Aufgrund der niedrigen Spezifität bei sehr hohen Sensitivität wird die Sinnhaftigkeit eines routinemäßigen Einsatzes der MR-Mg zur präoperativen Abklärung angezweifelt. Mit dem Ziel die Nachresektionsrate in unserem Brustgesundheitszentrum zu senken und zur genauen Planung des operativen Procedere, erhielten alle Patientinnen mit der stanzbioptisch gesicherten Diagnose eines Mammacarcinoms/DCIS seit Oktober 2009 routinemäßig eine MR-Mg.

Methodik. Durch die Evaluierung der vorhandenen Daten soll gezeigt werden, ob eine routinemäßige Durchführung der MR Mg sinnvoll ist und bei wie vielen Patienten durch die MR Mg eine Änderung des operativen Procedere stattgefunden hat, insbesondere mit den Fragestellungen: kontralaterales CA, Multizentrizität, Resektionsausmaß, Nachresektion erspart?

Ergebnisse und Schlussfolgerungen. Dies ist eine retrospektive Analyse von 150-200 Patientinnen mit der stanzbioptisch gesicherten Diagnose eines Mammakarzinoms seit Oktober 2009-Oktober 2010. Ergebnisse werden am Kongress vorgestellt werden.

\section{P34}

\section{Lymphknotenentfernung in der}

Metastasenchirurgie - unnötige Fleißaufgabe oder dringliche Pflicht?

\section{G. Seebacher, S. Decker, B. Enderes, G. Kugler, T. Graeter}

Klinik Löwenstein, Löwenstein, Germany

Grundlagen. Die Lymphknotenentfernung in der Metastasenchirurgie ist vielerorts noch kein akzeptiertes Routineverfahren. Auf der Suche nach Standards und zur Qualitätssicherung haben wir unsere Ergebnisse in der Metastasenchirurgie retrospektiv aufgearbeitet.

Methodik. Im Zeitraum 2006 bis 2009 unterzogen sich 217 Patienten (111 Männer, 106 Frauen) im Alter von 29 bis 81 Jahren einer Metastasenresektion. Diese Eingriffe wurden in einer retrospektiven Untersuchung aufgearbeitet und in Hinblick auf die Lymphknotenentnahme analysiert.

Ergebnisse. Bei 334 Operationen erhielten $38 \%$ der Patienten $(n=84)$ Mehrfacheingriffe. $76,9 \%$ aller Eingriffe wurden über Thorakotomie, $18,3 \%$ über Minithorakotomie durchgeführt und 4,8\% mittels VATS. In $55,5 \%$ der Eingriffe $(n=182)$ wurde mit Laser operiert. Das Lymphknotenstaging im Rahmen aller Erst- und Zweiteingriffe ergab eine N0 Situation in 67,6\% ( $n=198$ ) sowie einen positiven Lymphknotenbefall in $11,95 \%(n=35)$. Nach Tumoren getrennt zeigt sich bei Patienten, mit colorektalem Karzinom eine N0 Situation in $77,7 \%(n=73)$, ein positiver Lymphknotenbefall in 5,3\% $(n=5)$. Für das Nierenzellkarzinom zeigte sich ein etwas erhöhter metastatischer Lymphknotenbefall mit $16,7 \%(n=8)$ bei $64,3 \%$ N0 $(n=27)$. Bei Patienten mit Mammakarzinom zeigte sich ein metastatischer Lymphknotenbefall in $29,2 \%(n=7)$ sowie in $56,2 \%$ $(n=13)$ ein N0 Staging.

Schlussfolgerungen. Inwieweit eine radikale Lymphknotenentfernung therapeutischen Wert hat ist unsicher. Zumindest für die weitere Therapieplanung bringt die Lymphadenektomie zusätzliche Informationen.

\section{P35}

\section{Papilläres Schilddrüsenkarzinom bei tumorfreier Schilddrüse}

\section{G. Federmann, C. Hessler}

Klinik für Allgemein- und Visceralchirurgie, Bassum, Germany

Bei einem 75 jährigen Patienten trat eine Lymphknotenschwellung im lateralen Halsdreieck rechts auf. Die Lymphknotenentnahme ergab die Diagnose eines papillären Schilddrüsenkarzinoms. Die daraufhin nochmalige Durchuntersuchung mittels Sonographie, Szintigramm und CT konnte keinen Tumor in Schilddrüse oder oberen Mediastinum respektive im Halsbereich feststellen. Die Operation fand eine äußerlich unauffällige Schilddrüse, es wurde eine Thyreodektomie sowie eine Lymphknotendissektion durchgeführt. Die histologische Aufarbeitung ergab eine völlig tumorfreie Schilddrüse, die nochmalige Aufarbeitung des Lymphknotenmaterials ergab die Diagnose eines papillären Schilddrüsenkarzinoms mit partieller plattenepithelialer Differenzierung. Der Patient wurde zur Radio-Jod-Therapie weitergeleitet. 18 Monate nach der Therapie war der Patient wohlauf und rezidivfrei. Aufgrund der histologischen Befunde handelt es sich am ehesten um ein Schilddrüsenkarzinom auf dem Boden ektopen Schilddrüsengewebes in einem Lymphknoten oder den Resten einer lateralen Halszyste. 


\section{Varia}

\section{P36}

\section{Ultraschallgestützte und EUS-gestützte Therapie des „Haemosussus pancreaticus“}

\section{U. Will ${ }^{1}$, A. Müller ${ }^{1}$, F. Füldner', I. Wanzar' ${ }^{1}$, F. Meyer ${ }^{2}$}

${ }^{1} \mathrm{SRH}-$ Waldklinikum, Klinik für Gastroenterologie, Gera, Germany; ${ }^{2}$ Universitätsklinikum A.ö.R., Klinik für Allgemein-, Viszeral- \& Gefäßchirurgie, Magdeburg, Germany

Anhand von zwei außergwöhnlichen Fällen wird der Stellenwert der Sonographie \& Endosonographie (EUS) in der Diagnostik/Therapie des „Haemosuccus pancreaticus“ beschrieben. (1) 40-jährige Patientin mit wiederholten Blutungsepisoden im oberen GI-Trakt \& hämorrhagischen Schock (Anamnese: Chronische Pankreatitis, Pseudozysten). Die konventionelle Abdomen- \& Duplexsonographie zeigten ein kleines $(1 \mathrm{~cm})$ Pseudoaneurysma im Pankreasschwanz (Ursache des „Haemosussus pancreaticus“). Unter Notfallbedingungen wurden sonographisch gestützt $2 \mathrm{ml}$ Fibrinkleber bzw. bei frustraner interventioneller Angiographie im Intervall $2 \times 2 \mathrm{ml}$ eines Lipiodol-Histoacryl-Gemisches ins Pseudoaneurysma appliziert mit kompletter/permanenter Blutstillung. Nachfolgende Kontrollen mittels Duplexsonographie demonstrierten die suffiziente Exklusion des Pseudoaneurysmas ohne nachweisbare Perfusionstörung des Milzparenchyms. (2) Patient mit chronischer Pankreatitis \& Pankreasgangstenose mit Einweisung zum elektiven Stentwechsel. Nach Stententfernung arterielle Blutung aus dem Pankreasostium. Verschluss der Papille notfallmäßig mit Clips. Anschließend durchgeführte EUS zeigte das Pseudoaneurysma im Pankreas. Punktion \& Installation von $2 \mathrm{ml}$ Fibrin in gleicher Sitzung. Mit dieser Technik Aneurysmaverschluss, allerdings Perfusionsstörungen der Milz mit Milzinfarkten durch Fibrineinschwemmungen in Milzarterie. Weiteres Vorgehen konservativ - bisher keine erneuten Blutungen (Aneurysma nicht mehr detektierbar, Milzinfarkte haben sich konsolidiert).

Zusammenfassung. Es handelt sich hier um die ersten Fallberichte einer erfolgreichen Blutstillung bei „Haemosussus pancreaticus" durch sonographische und EUS-Intervention. Die Methodik ist anspruchsvoll und mit Problemen behaftet (Milzinfarkte), allerdings als Notfallintervention bei hämodynamisch instabilen Patienten oder Hochrisikopatienten durchaus geeignet.

\section{P37}

Dünndarmvolvulus - diagnostisches \& therapeutisches Management einer chirurgischen Rarität des akuten Abdomens im Erwachsenenalter mit befundspezifischer moderner Bildgebung per impressiver CT-basierter Videosequenz

\section{S. Balgon', C. Wex ${ }^{2}$, L. Rapp ${ }^{3}$, H. Lippert ${ }^{2}$, F. Meyer ${ }^{2}$ \\ ${ }^{1}$ Universitätsklinikum A.ö.R., Klinik für Plastische, Wiederherstellende \& Handchirurgie, Magdeburg, Germany; ${ }^{2}$ Universitätsklinikum A.ö.R., Klinik für Allgemein-, Viszeral- \& Gefäßchirurgie, Magdeburg, Germany; ${ }^{3}$ Universitätsklinikum A.ö.R., Klinik für Radiologie \& Nuklearmedizin, Magdeburg, Germany}

Grundlagen. Bericht mittels exemplarischer Fallpräsentation über außergewöhnlichen Casus eines 67jährigen Patienten mit Computertomographie (CT)-bildgebend gesichertem, neuartig durch Schnittbildfolge ergänztem sowie operativ bestätigtem Dünndarmvovlulus.

Methodik und Ergebnisse. Jetzige Anamnese - abdomineller Vernichtungsschmerz \& Obstipation (Notfallvorstellung). Klinisch: Lokale Abwehrspannung \& Druckschmerz im Unterbauch (therapierefraktäre Schmerzsymptomatik) bei wechselnden Darmgeräuschen in allen 4Quadranten im Sinne des akuten Abdomens (unauffällige Paraklinik/Abdominalsonographie). Abdomen-CT: Eindrucksvoll durch videosequenztechnische Transversal-Scans untersetzte \& letztlich beweisende „Twister“-ähnliche Struktur mit spiralförmig angeordneten Verdichtungen. Prompte Laparotomie: Bestätigung des V.a. Dünndarmvolvulus - umgehende essenzielle Detorquierung. Nebenbefundlich: (i) Sigma elongatum, (ii) mäßig ausgeprägtes Coecum mobile, (iii) überlanges (,durchhängendes“) Colon transversum. Ohne erkennbaren Auslöser war am ehesten von primärem Dünndarmvolvulus auszugehen, begünstigt durch überlange Mesenterialwurzel. Replatzierung des detorquierten reperfundierten Dünndarmabschnittes (Ausdehnung: Ca. $80 \mathrm{~cm}$ ) mittels Dünndarmfältelung. Postoperativ: Keine Komplikationen - Entlassung: 11. postoperativer Tag.

Schlussfolgerungen. Die auch im klinischen Alltag unter Notfallkriterien fast „real time“ erzielbare Bildbearbeitung ließ rasch/zweifelsfrei die korrekte Diagnose stellen \& adäquate Therapie mittels zügiger Laparotomie zur Darmdetorquierung einleiten. Die CT unterstreicht schon frühzeitigen Stellenwert im differenzialdiagnostisch abklärenden Diagnostikgang beim unklaren/akuten Abdomen, u.a. mit Befund-assoziierter Akzentuierung durch weiterführende zeitnahe Bildbearbeitung entsprechend gegebener technischer Potenzen, um für den Patienten die diagnostische Sicherheit zu erhöhen, kostbare Zeit im therapeutischen Management zu sparen \& damit die Gesamtprognose effektiv zu verbessern. 


\section{P38}

\section{Obturatorius Hernie - Die Hernie der „kleinen alten Damen“ - ein Fallbericht}

\section{R. J. Stadlhuber, A. Dubecz, M. Schweigert, H. J. Stein}

Klinik für Allgemein-, Viszeral und Thoraxchirurgie, Klinikum Nürnberg Nord, Nürnberg, Germany

Die Obturatorius Hernie ist eine diagnostische Herausforderung, die oft erst im OP gestellt wird. Die Inzidenz beträgt $0,5 \%$ bis $1,4 \%$.

Wir berichten über eine 96-jährige Patientin die mit stärksten abdominellen Schmerzen sowie rezidivierendem Erbrechen über mehrere Tage eingewiesen wurde. Klinisch zeigte sie das typische Bild einer Darmmotilitätsstörung. Die Computertomographie zeigte einen Dünndarm-Ileus als Folge einer Dünndarmherniation durch das offene linke Foramen obturatorium.

Die Notfalllaparatomie bestätigte die CT-graphische Diagnose und der Defekt wurde mit einem Vicryl-Netz verschlossen.

Das Patientengut umfasst fast ausschließlich Damen über 80 als Folge eines erschlafften Beckenbodens. Bei älteren kachektischen Patientinnen mit Ileus und/oder unspezifischen Schmerzen im Bereich der Hüfte, ohne bauchchirurgische Eingriffe in der Anamnese, sollte immer an eine Obturatorius Hernie gedacht werden.

Exakte Diagnose, unverzügliche operative Versorgung und suffizienter Verschluss der Hernie sind die Hauptprognosefaktoren eines positiven Therapieergebnisses.

\section{P39}

\section{Laparoskopische Kolon-/ Rektumchirurgie: Ergebnisübersicht aus dem AKH Linz}

\section{A. Shamiyeh, K. Szabo, A. Pentsch, E. Huber, M. Schreiner, W. U. Wayand}

Linzer Ausbildungs- und Forschungsinstitut für Chirurgie und Onkologie sowie Ludwig Boltzmann Institute for operative laparoscopy, Linz, Austria

Grundlagen. In Österreich werden aktuell ca. $20 \%$ aller Kolonresektionen minimalinvasiv durchgeführt, weniger als $1 \%$ mittels neuester Verfahren wie Single Inzision, transvaginaler, zuletzt auch transrektaler Technik. Neue Techniken müssen sorgfältig evaluiert werden und dürfen für den Patienten kein erhöhtes Risiko darstellen. Im Rahmen unserer Qualitätssicherung führen wir seit über 11 Jahren ein prospektives Register, in dem alle Patienten, die einer laparoskopischen Kolon-/Rektumresektion zugeführt werden, erfasst werden. Die aktuellen
Daten sollen als Poster und Abstrakt zugänglich gemacht werden und stellen einen nationalen Richtwert für Morbidität und Mortalität dar.

Methodik. Von 5/1999 bis 12/2010 wurden 518 Patienten (m 249, w 269, mittleres Alter 62, range 22-92) operiert. 333 aufgrund einer Divertikulitis (157 gedeckt perforiert), 124 Patienten wegen eines Karzinoms oder Tumors. Die übrigen 61 wegen benigner Erkrankungen wie u.A. wie Endometriose, Prolaps, Perforation.

Ergebnisse. Bei 23 Patienten wurde keine Anastomose angelegt. Von den restlichen 495 hatten 11 eine Anastomoseninsuffizienz (2,2\%). Die Gesamtmorbidität beträgt $16,9 \%$, Die Mortalität beträgt $0,77 \%(n=4)$. Die häufigsten Komplikationen waren Wundinfekt (19), postoperative Anämie (11), Anastomosenblutung (9), postoperativer Ileus (8) und Anastomosenstenose (6). Die Konversionsrate beträgt $1,35 \%(7)$.

Schlussfolgerungen. Morbidität und Mortalität halten dem internationalen Vergleich stand. Durch Implementierung neuer nicht evaluierter Techniken sollte es zu keiner Verschlechterung kommen.

\section{P40}

\section{Hollunderzweig-Choledocholithiasis: ein Fallbericht}

\author{
A. U. Rothe ${ }^{1,2}$, M. Sedlak ${ }^{3}$, W. Wayand ${ }^{1,2}$ \\ ${ }^{1}$ Il. Chirurgische Abteilung, AKH Linz, Linz, Austria; \\ ${ }^{2}$ Ludwig Boltzman Institut für Operative Laparoskopie, \\ Linz, Austria; ${ }^{3}$ II. Interne Abteilung, AKH Linz, Linz, Austria
}

Ein 64-jähriger Patient wurde bei Verdacht auf Sigmadivertikulitis zur weiteren Abklärung stationär aufgenommen. Die durchgeführte Abdomen-Computertomographie bestätigte die Verdachtsdiagnose - nebenbefundlich zeigte sich eine Choledocholithiasis. Dieser Zufallsbefund - bei einem Aufnahmebilirubin von 1,3 mg/dl zunächst nur inkomplett obstruierend - führte in der Verlaufskontrolle zu einem Bilirubinanstieg auf $5,8 \mathrm{mg} / \mathrm{dl}$, so dass der Entschluss zur ERCP unter laufender konservativer Divertikulitistherapie fiel.

In der ERCP zeigte sich überraschend ein durch die Papilla Vateri ins Duodenallumen hineinragender Fremdkörper, der nur mühsam nach Nadelpapillotomie mitsamt einem Konglomerat an Choledocholithen geborgen werden konnte. Bei näherer Betrachtung des ungewöhnlichen Bergungsgutes, enpuppte sich der Gegenstand als verzweigtes Ästchen von $4 \mathrm{~cm}$ Länge.

Das Präparat wurde zur weiteren Analyse an das Institut für Dendrochronologie der Universität für Bodenkultur Wien geschickt, mit dem Ergebnis, dass es sich hierbei mit großer Wahrscheinlichkeit um einen Hollunderzweig handelte.

In einer Durchsicht der Literatur wurden unter anderem chirurgische Clips, Hühnerknochen, Fischgräten, Tomatenschalen, Gewehrkugeln und Granatsplitter 
als Ursachen für eine lithogene Choledochusobstruktion beschrieben - die Verlegung durch einen Hollunderzweig wurde bis dato noch nicht publiziert.

Der Patient wurde mit dem Ergebnis konfrontiert und gab an, immer wieder selbstgemachten „Hollerröster“ eine oberösterreichische Spezialität - zu essen.

\section{Thoraxchirurgie}

\section{P41}

\section{Aortokoronare Bypassoperation bei Patienten mit dialysepflichtiger Niereninsuffizienz}

\section{J. Sirch, S. Pfeiffer, T. Fischlein}

Klinikum Nürnberg, Klinik für Herzchirurgie, Nürnberg, Germany

Grundlagen. Dialysepflichtige Koronarpatienten stellen eine Hochrisikogruppe für die Bypasschirurgie dar. Die Daten wurden retrospektiv mittels Aktensichtung sowie Fragebogen an Pat. und Hausarzt bzw. Dialysezentrum ermittelt.

Methodik und Ergebnisse. Im Zeitraum von April 1994 bis Dezember 2010 wurden 8546 Patienten isoliert an den Koronararterien operiert, bei 141 (1,7\%) davon bestand zum Operationszeitpunkt eine dialysepflichtige Niereninsuffizienz, die mittlere Dauer der Dialyse war 46,6 Monate (1-275). Das Alter betrug 63,9 (37-83) Jahre, 112 Patienten waren männlich.

Der durchschnittliche Nachbeobachtungszeitraum war 43,2 Monate. Die 30 Tage-Letalität betrug 5,0\% (7 Pat.), der Aufenthalt auf der Intensivstation postoperativ war 10,1 (1-80) Tage, der Krankenhausaufenthalt insgesamt 22,5 (6-133) Tage. Die statistische Überlebensrate betrug nach einem Jahr $81 \%$, nach 2 Jahren $73 \%$ und nach 5 Jahren $51 \%$. Die subjektive Lebensqualität beschrieben 96 der noch lebenden Pat. (69\%) besser als vor der Operation, 39 Pat. (28\%) unverändert und bei 4 Pat. (3\%) war es zu einer Verschlechterung gekommen. Eine Nierentransplantation war bei 17 Pat. vorgesehen und bei 6 im Verlauf auch durchgeführt.

Schlussfolgerungen. Die Daten zeigen, dass Patienten, die lange Zeit dialysepflichtig sind, für eine Bypassoperation ein höheres Risiko haben. Die Langzeitergebnisse und die subjektiv empfundene verbesserte Lebensqualität bei über $70 \%$ aller operierten Patienten rechtfertigen eine koronare Bypassoperation auch bei dieser Hochrisikogruppe.

\section{P42}

\section{Die seltene Ursache einer Lungenhernie Fallbericht, Pathogenese und Literaturübersicht}

\author{
W. Schreiner, O. Oster, A. Zdrojek, H. Sirbu \\ Thoracic Surgery, University hospital, Erlangen, Germany
}

Grundlagen. Die spontane interkostale Lungenhernie ist ein seltenes Phänomen. Weltweit wurden insgesamt 25 Fälle publiziert.

Methodik und Ergebnisse. 59 jähriger adipöser Patient berichtete vom heftigen Hustenanfall mit einschießenden thorakalen Schmerzen vor 3 Monaten. Zeitgleich aufgetretene laterale Vorwölbung war im Verlauf progredient. Beim Valsava- Manöver konnte gravierende Protrusion provoziert werden. Palpatorisch bestand eine deutliche Distension im Bereich des linken Rippenbogens und ein Volet mobile mit paradoxer Atmung. Ct-morphologisch waren große Anteile des linken Unterlappens nach anterolateral verlagert. Technik: Über die tiefe Thorakotomie im 7. ICR erfolgte die Darstellung der Bruchpforte. Die Interkostalmuskulatur war vollständig zerrissen. Nach Einbringung von mehreren Perikostalnähten wurde die Rippenbogenrekonstruktion und Stabilisierung mit Drahtcerclagen durchgeführt. Zusätzlich zur Sicherung des Interkostalraums erfolgte die Implantation eines Marlexpatches $18 \times 18 \mathrm{~cm}$ doppelschichtig.

Schlussfolgerungen. Die „spontane“ Herniation ist mit plötzlichem Anstieg des intrathorakalen Drucks verbunden. Entsprechend den 25 publizierten Fällen handelt es sich um Husten, Singen oder Heben von Lasten. Anteriore Thoraxwand ist- infolge der relativen Schwäche der ventralen Interkostalmuskulatur einerseits und der Stabilisierung durch extrathorakale Muskulatur im posterioren Bereich anderseits- die Prädilektionsstelle sowohl für traumatische und spontane Hernien. Infolge geringer Tendenz zur spontanen Regression sehen wir die Indikation zur raschen operativen Therapie. Perikostale Fixierung im gesamten instabilen Bereich ist biomechanisch wichtig.

\section{P43}

Erste Erfahrungen mit der implantierbaren Infusionspumpe (Lenus Pro)

mit 4wöchigem Auffüllzyklus bei einer

Patierntin mit pulmonal arterieller

Hypertonie (PAH) zur Verabreichung

von Treprostinil - ein Fallbericht

\author{
R. Steringer-Mascherbauer ${ }^{1}$, V. Eder ${ }^{1}$, C. Ebner ${ }^{1}$, \\ J. Niel ${ }^{1}$, J. Nesser ${ }^{1}$, R. Függer ${ }^{2}$ \\ ${ }^{1}$ Abt für Kardiologie, KH der Elisabethinen, Linz, Austria; ${ }^{2} \mathrm{Abt}$ \\ für Chirurgie, KH der Elisabethinen, Linz, Austria
}


Grundlagen. Treprostinil als subkutane Dauerinfusion ist eine etablierte Therapie der schweren PAH. Lokale Schmerzen und Infektionen führen aber in $15 \%$ zum Therapieabbruch. Erste Erfahrungen mit einer implantierten Pumpe liegen aus Deutschland vor, sind aber in der Praxis durch einen 20tägigen Wiederbefüllzyklus limitiert.

Methodik. Wir berichten über die weltweit erstmalige Implantation einer im 28-Tage-Zyklus wiederbefüllbaren Infusionspumpe zur kontinuierlichen Treprostiniltherapie bei PAH.

Ergebnisse. Bei der 70jährigen Patientin wurde 2009 eine PAH diagnostiziert (bei Aufnahme NYHA-Stadium IV, mPAP $62 \mathrm{mmHg}$, CI $1,38 \mathrm{l} / \mathrm{min} / \mathrm{m}^{2}$, NT-proBNP $1561 \mathrm{ng} / \mathrm{l})$. Eine subkutane Treprostinildauertherapie über eine externe Infusionspumpe zeigte ein gutes Ansprechen mit Verbesserung der rechtsventrikulären Funktion und des klinischen Stadiums, limitiert durch Schmerzen und eine therapiepflichtige subkutane Infektion im Infusionsbereich. Im September 2010 erfolgte schließlich die Implantation der 28-Tage- Lenus Pro ${ }^{\circledR}$ Pumpe. Nach Punktion der rechten Vena subclavia und typischer Einbringung des zentralvenösen Katheters erfolgte die Implantation der Pumpe in eine subkutane Tasche im rechten Oberbauch. Die Pumpe wurde an der Bauchdeckenfaszie fixiert und nach Tunnelierung mit dem zentralvenösen Katheter konektiert. Bis auf ein Serom war der postoperative Verlauf komplikationslos, die Patientin ist unter Treprostinil stabil gebessert.

Schlussfolgerungen. Die bisherigen Erfahrungen zeigen eine gute Praktikabilität des vierwöchigen Befüllzyklus bei Senkung der therapieassoziierten Morbidität gegenüber der subkutanen Infusion.

\section{Unfallchirurgie}

\section{P44}

The Targon ${ }^{\circledR}$-PH Nail as an internal fixator for unstable capital humeral fractures in the elderly patient: a retrospective study of 39 patients

L. Mica ${ }^{1}$, T. Fröhlich ${ }^{2}$, A. Schwaller ${ }^{3}$, A. Vollenweider ${ }^{3}$, M. Rancan ${ }^{4}$

${ }^{1}$ Trauma Surgery University Hospital Zürich, Zürich, Switzerland; ${ }^{2}$ Chirurgie Spital Lachen, Lachen, Switzerland; ${ }^{3}$ Chirurgie Spital Männedorf, Männedorf, Switzerland;

${ }^{4}$ Chirurgie Spital Triemli, Zürich, Switzerland

The proximal humeral fracture occurs often especially in elderly patients suffering osteoporosis. Minimally inva- sive and exercise stable fixation with the Targon ${ }^{\circledR}$-PH proximal humeral nail of the fracture may lead to a good recovery and social integration. 39 patients with unstable humeral head fractures were included into this study. The Constant Murley Score was used to evaluate functionality of the shoulder. One way ANOVA analysis was used according to the numbers of groups. The data were given as mean \pm SEM and significant if $\mathrm{p}<0.05$. The mean age was $74.9 \pm 1.8$ years. The last postoperative check up was made after $26.1 \pm 2.2$ months. The subjective parameters of the Constant Murley Score were significantly lowered in both the female and male population compared to the healthy side (female: $30.0 \pm 1.1$ vs. $33.7 \pm 0.7 ; p<0.05$; male: $30.3 \pm 1.4$ vs. $34.0 \pm 0.7 ; p<0.05)$. The objective parameters were significantly lowered $(34.5 \pm 2.0 \mathrm{vs}$. $45.2 \pm 1.1 ; p<0.001$; female and $37.7 \pm 5.4$ vs. $50.6 \pm 1.7$; $p<0.05$; male). The antegrade angular stable nailing of proximal humeral head fractures may represent a method for early social integration of the elderly patients.

\section{P45}

\section{Violence under the influence of alcohol is age-related, a multiple logistic regression analysis of 300 patients}

\section{Mica, L. Oesterle, G. A. Wanner, H. Simmen, C. Werner}

Trauma Surgery Zürich, Zürich, Switzerland

Rising violence among drunken young people is being reported by different media. Sveral surveys confirmed this fact. There are almost no clinical data analysing the correlation between alcoholic intoxication, age, gender and violence. The aim was to evaluate alcohol, age or gender is independent predictors to commit violence.

In a prospective study between 1.1.2009 and 31.12.2009 at the Trauma Division of the University Hospital of Zürich 300 patients were enrolled in the study with an average age of $36.9 \pm 16.9$ years. The inclusion criteria was a blood alcohol content of $\geq 10 \mathrm{mmol} / \mathrm{L}$ and any traumatic injury. Data were compared with MannWhitney test and $\chi^{2}$-test. The data were considered as significant if $p<0.05$. Predictive ability was evaluated by using receiver operating characteristic (ROC-) curve. Independent predictors were analyzed by logistic regression analysis. The data revealed a significant gender $(p<0.001)$ and age $(29.3 \pm 11.9$ vs. $45.2 \pm 17.5$ years, $p<0.001)$ dependent violence with no correlation to blood alcohol content. Logistic regression analysis revealed gender and age as an independent predictor for violence under the influence of alcohol. Age controled pour out of alcoholic drinks seems to be the best preventive way to reduce alcohol induced violence in young male. 



\section{Author index}

Bold numbers indicate the presenting authors.

\section{A}

Abrahim, A. 297, 298, 301, $302,385,389$

Abugameh, A. 089

Adambegan, M. 383

Adamina, M. 262, 325

Aharinejad, S. 122

Aigner, C. 053, 094, 280

Aigner, F. 120, 340

Aigner, I. 376

Ainoedhofer, H. 123

Alavian, M. 080

Albokrinov, A. 245

Albrecht, K. 122, 149

Ali-Abdullah, S. 298, 301, 302, 389

Aliabadi, A. Z. 280

Alidzanovic, L. 170, P24

Altmann, P. 280

Ammann, K. 363

Anegg, U. C. P20

Anelli Monti, M. 186

Angleitner, P. 247

Ankersmit, H. J. 019, 043, 044, 045, 189, 190

Antonietti, E. 261

Ardelean, M.-A. A. P16

Ardelt-Gattinger, E. 303

Arndt, S. 268

Arneitz, C. 292

Arni, S. 021

Arnold, B. N. 158

Asari, R. 419

Ashraf, M. 076

Aspalter, M. 102, 322

Aszmann, O. 036, 038, 048, 136,350

Aufschnaiter, M. 067, 091, 339, P33

Augustin, F. 103, 112, 120, 236, 237, 238, 239

Ausch, C. 166, 415

Autschbach, R. 379

\section{B}

Bach, F. 074, 075

Bacher, B. 376

Bachleitner-Hofmann, T. 165

Bale, R. 421

Balgon, S. P37

Bartel, T. 081, 083

Bartnik, A. P27

Bartsch, R. 165, 252, 416

Baschata, M. 066

Bastian, F. 413

Baubin, M. A. 032

Bauer, J. 148, 249a

Bauer, R. P26

Bauer, T. 349

Bauernhofer, T. P30

Baumann, S. 247, 248, 289

Bäumel, D. 197

Baumgartner, A. 043, 124, 189,190

Baur, M. 413

Beck, J. 351

Beckerhinn, P. 305

Beham, A. P25

Benedix, F. P22
Benhidjeb, T. 108

Benkoe, T. 247

Beran, C. 116

Beran, E. $126,186,378$

Berényi, E. 045

Berger, K. P17

Berghold, A. 128

Bernhard, D. 024

Bernhardt, G. A. P06

Beyer, M. 134

Bibeau, F. 165

Biebl, M. 010, 046, 061, 097, $103,111,222$

Bieglmayer, C. 419

Bigenzahn, S. 168

Bijak, M. 380

Binder, C. 342

Binder, M. 426

Birkfellner, F. 130, P01

Birner, P. 167, 414

Birsan, T. P03

Bischof, G. 056

Bischofberger, S. 227, 325

Bittner, R. 232

Bodner, J. 103, 112, 237, 238, 239

Boesmueller, C. 103

Bohdjalian, A. 258, P03

Bonaros, N. 022, 081, 083, $122,145,146,184,375$

Bonatti, J. 146, 375

Borova, O. 291

Bösch, F. 075

Bösmüller, C. 046

Bracko, M. P28

Brand, J. 118, 127

Brandacher, G. 020, 024, 071, 072, 125

Braunschmid, T. 413

Brostjan, C. 169, 170, P24

Brotzge, L. 261

Brugger, E. 011

Brugger, P. 169

Brunialti, M. 418

Brunner, W. 007, 009, 012

013, 057, 059, 060, 114,

$115, \mathbf{1 5 7}, 221,224, \mathbf{2 2 5}$,

$226,315,390$

Buchner, S. 241

Buhr, H. J. 366

Bures, C. 420

Burgard, G. 175

Büschel, P. 391, P10, P13, P31

Buxhofer-Ausch, V. 166, 415

\section{C}

Cadariu, D. 305

Cajlakovic, M. 126

Cakar-Beck, F. 338

Cardini, B. 024, 071

Cavadas, P. 020

Celeiro, J. 325

Cerwenka, H. 382

Chalupar, Q. D. 417

Chan, Y.-W. P27

Chevtchik, O. 181, 182, 183

Chiari, F. 028

Chooklin, S. P11

Ciovica, R. 306, P02
Cohnert, T. 326,327

Coosemans, W. 094

Croner, R. 281

Cross, C. 143

Curcic, P. 126

Cziupka, K. 155

D

Dabernig, W. 018

Dacar, D. 378

Dandel, M. 140

Dapunt, E. 064

Dauser, B. 316, P08

De Leyn, P. 094

Decaluwe, H. 094

Dechantsreiter, G. 088

Decker, G. 094

Decker, S. P34

Delko, T. P05

Deluggi, S. 294

Demmer, J. $\mathbf{0 8 0}$

Deutinger, M. 039, 252

Diemath, H.-E. 204

Diesner, S. C. P03

Dietl, H. 048

Dietz, H. 088

Dinnewitzer, A. 260, 337

Dirnberger, J. 228

Djedovic, G. 349

Dlask, J. 328

Dollinger, C. 404

Dominkus, M. 348

Doodnath, R. 191

Dubecz, A. 286, 365, 392. P38

Dubsky, P. $\quad 168,416$

Duess, J. W. 191

Duma, A. 323

Dünser, M. 101

Dunst, C. M. 153

Dvorakevich, A. 245, 295

Dzilic, E. 124

\section{E}

E. Afshar, D. 252

Ebner, C. P43

Ebner, M. 076

Eder, V. P43

Egger, M. 380

Eichmann, E. $\quad$ P30

Eisenbach, C. 075

Eisner, F. P30

Emmanuel, K. 018, 102

End, A. 235

Enderes, B. P34

Ennemoser, T. 032

Ensat, F. 207, 351

Ensinger, C. 195

Entner, T. 040

Enzinger, S. $\mathbf{0 3 3}$

Erne, B. V. 021

Ewers, R. 029

Exner, R. 168, 284

\section{F}

Fartacek, R. 248

Fasching, G. 292, 296, 404
Federmann, G. $\quad$ P35

Feichtinger, E. 143

Feichtinger, M. 030

Felberbauer, F. X. 258

Fenz, C. 305

Fenzl, G. P09

Ferlitsch, A. 282

Fetzner, U. K. P13

Feuchtner, G. 145, 146

Fiegl, K. 090

Fiegl, M. 237

Fink, M. 147

Fischbach, N. P09

Fischlein, T. 054, 085, P4

Fischler, N. $181,182, \mathbf{1 8 3}$

Fitzal, F. $\quad$ 168, 416

Fitzka, T. 337

Folkmann, S. 082, 084, 086 185,380

Fortelny, R. H. 052, 062 ,

063, 118, 127, 232

Foussek, C. 186

Frass, R. 007, 009, 012, 013, 057, 059, 060, 114, 115 .

157, 225, 315, 390

Freilinger, W. P14

Frey, E. 035, 049

Frey, M. 019, 035, 036, 037 038, 040, 048, 049, 136, $251,255,350$

Friedrich, G. 081, 083, 145

Friesenbichler, J. P25

Fröhlich, T. P44

Fröschl, U. 231, 313

Fruhmann, J. 326

Fry, L. C. 391

Függer, R. 006, 058, 231,

313, P43

Füldner, F. P36

Fuller, B. P27

Funovics, P. T. 348

Furtmüller, B. 098, P32

\section{G}

Gabor, S. E. $\quad 095,133$

Gadenstätter, M. 306, P02

Gaggl, A. 028, 033, 034

Gangl, O. 006, 058, 231, 313

Garriboli, M. 191

Gaßner, I. 194, 195, 196

Gassner, R. 032

Gehwolf, P. 074, 075, 242, 243, 338

Geier, K. 121

Gellersen, S.-O. 260

Gerhard, S. 032

Germer, C.-T. 017

Gerzanic, L. 208

Ghaffari, S. P08

Giesinger, J. 093, 336

Gingert, C. 262

Girardi, D. 228

Giretzlehner, M. 228

Girsch, W. 346, 347, P15

Glaser, K. S. 052,418

Glaser, K. 062, 063, 090, 127

Glöckler, M. 343

Gnant, M. 099, 168, 169, 416 Göbl, S. 420 
Gorkiewicz, G. 377

Gorlitzer, M. 084, 086, 179, $185, \mathbf{3 8 0}$

Gössler, A. 292, 296

Götzinger, P. 099

Grabenwöger, M. 082, 084, 086, 179, 185, 380

Graeter, T. P34

Grauhan, O. 140

Greiner, A. 051, 379

Grill, A. 305

Grimm, M. 017, 022, 042, $055,081,083,122,139$,

$144,145,181,182,183$ 184,374

Grißmann, T. K. 098, P32

Grommes, J. 051, 379

Gröne, J. 366

Groß, C. 080

Gross, C. 141

Gruber-Blum, S. 052, 118, 127

Grünberger, B. 170, P24

Grünberger, T. 069,170 , 282, P24

Gschwandtner, E. 383

Gyöngyösi, M. 044, 045

$\mathrm{H}$

Haberlik, A. 176

Hachleiner, J. 033

Hachleitner, J. 034

Hacker, S. 019, 045

Hackl, C. 101

Hackl, W. 137, 312

Hader, H. 123

Hager, J. 192, 193, 194, 195 196, 243

Hager, T. 192, 194, 195, 196

Haid, A. 165

Haider, T. 019

Halloul, Z. P10, P12, P13

Hamilton, G. 166

Hangler, H. B. $\quad 055,139,144$ 374

Hanika, M. 381, P19

Happak, W. 136

Harrer, C. 304

Hartert, M. 089

Haschke-Becher, E. 376

Haslik, W. 136

$\mathrm{Ha}$, H.-J. P12

Hasun, M. 023, 043, 124, 189, 190

Hauer, A. P29

Haunold, I. 223, 259

Hausbrandt, P. A. 198

Häussler, B. 193, 194, 196, 243

Hautz, T. $\quad 020$

Havrysh, Y. 109

Haxhija, E. Q. 197

Hay, D. P10

Hechenleitner, P. 193, 194 195, 243

Heck, D. P33

Heinz, A. $055, \mathbf{0 8 1}, \mathbf{0 8 3}, 139$ 144,374

Helfgott, R. P33

Helml, G. 254, 256

Helmreich, V. 235

Hengster, P. 070

Herberger, B. 170, P24

Herbst, F. 316, P08

Herczeg, M. 048

Hermann, M. 070, 071, 233, 383,420
Herold, M. 054

Herrnhof, W. 261

Hessler, C. P35

Hetterle, R. 186

Hetzer, R. 140, 180

Hiemann, N. 140

Hierm, D. 335

Hintringer, T. 250, 253

Hinzmann, M. 054

Hirschl, K. 035, 040, 049

Hladik, M. 207, 207, 351

Hoblaj, T. 135, 137, 312, 413

Hoetzenecker, K. 044, 045 , 189, 280

Hofauer, B. 145,375

Hofer, W. 006, 058

Hoffer, F. 305

Höfler, T. 234

Hohenberger, W. 281

Hold, A. 037, 350

Holfeld, J. 042

Hollinsky, C. 116

Hollinsky, S. 116

Höllwarth, M. 123, 191, 197, 198, 293

Holmer, C. 366

Hölzenbein, T. 322

Holzner, B. 093, 336

Horcher, E. 247, 248, 289

Hörletzberger, M. 326, 327

Horvath, M. 312

Horvath, Z. 168

Hotz, T. 150

Hötzenecker, K. 053

Hranat, O. P11

Huber, E. 329, P39

Huber, J. 006, 058

Huber, S. 187, 188

Huemer, G. M. 254, 256

Hufschmidt, M. 363

Hutter, J. 066, 241

I

Iberer, F. 087

Inci, I. 021

Inci, M. 023

Innerhuber, M. 141, 143

Ivancic, P. 030, 031

\section{J}

Jacobs, M. 051, 379

Jakesz, R. 416

Jakse, G. P21

Janczak, J. 156

Jara, M. 046

Jaschke, W. 192

Jensen-Jarolim, E. P03

Jesch, B. 167, 414

Jessl, J. 054

Jomrich, G. 167, 414

Jung, M. 017

Jungraithmayr, W. 021

Justich, I. 257

\section{K}

Käch, K. 150

Kaczirek, K. 282

Kafka, R. 093, 096, 336

Kafka-Ritsch, R. 196, 338, 417

Kaiblinger, J. 179

Kalak, N. 227
Kalinski, T. P31

Kamolz, L.-P. 136, 209

Kapp, K. P21

Kapral, S. 152, 323

Kärcher, H. 030, 031, 208

Kargl, S. 142

Karle, B. 136, 251, 255

Karnel, F. 137, 312

Karner, C. 347

Karner, J. 100, 135, 137,

$234,312,413$

Kasprian, G. 350

Kaufmann, C. 120

Keeling, I. 378

Kees, M. 298, 301, 302, 385

Kees-Belyus, M. 297

Kefurt, R. 258

Keller, K. 392

Kern, B. 072, 125

Khajanchee, Y. S. 158

Kienzl-Wagner, K. 010

Kiesslich, T. P30

Kilo, J. 055, 139, 144, 374

Kircher, S. 017

Kitzinger, H. B. 136, 251 , 255

Klapper, H. 065

Klaus, A. 061, 111, 222, 223

Klein-Franke, A. 192

Klepetko, W. 043, 044, 045 , $053,189,190,280$

Klimpfinger, M. 100

Klug, C. 029

Klug, R. 065, 342, P09, P29

Knauer, M. 165

Knez, I. 126

Kniepeiss, D. 068

Knosalla, C. 140

Kober, F. 383, 420

Koch, H. 426

Koch, T. M. P06

Kocher, A. 022, 122

Köckerling, F. 232

Kofler, M. 122

Kogler, P. $\quad 075,096,338$

Köhler, G. $\quad 067,339$

Koidl, C. 075

Kolivoshka, J. 295

Koller, M. 250, 253

Komoni, A. S. 197

Konstantiniuk, P. 326, 327

Kopp, T. P03

Kornigg, K. 084, 179

Köstler, T. P05

Kostron, A. 074

Koter, S. 326, 327

Kovanyi Holzer, B. 092

Kozuchowska, U. 223

Krabatsch, T. 180

Krafka, K. 296, 404

Krapf, C. 122

Krasny, C. P14

Krause, H. P12

Krause, R. 377

Kreibich, M. 124

Kremer, J. 023, 124

Krenkel, C. $\quad 033,034$

Kresse, A. 073

Kretschmer, N. P26

Kriegshäuser, G. 415

Kriwanek, S. 092, 166, 178, 304

Krois, W. 244

Kroker, S. P12

Kronberger, I. 338, 417

Kröpfl, A. 152

Krumnikl, J. 126

Kube, R. P22

Kubiena, H. $\quad$ 035, 040, 049 136

Kuess, A. 121

Kugler, G. P34

Kühbacher, C. 133

Kührer, I. 169

Kukucka, M. 180

Kurz, F. 091, 339

Küster, D. P23

Kuznetsov, A. 022

L

Lackner, C. P30

Lade, B. 130

Laimer, E. 061, 097, 222 240, 299

Lakkis, F. G. 020

Lammer, J. 035, 040, 049

Lampl, L. 134

Lancee, S. 261

Landin, L. 020

Landmann, D. 017

Landsiedl, A. 297, 298, 301, 302

Landsiedl, F. P14

Lang, G. 053, 280

Lang, R. 186

Langer, F. 258, 364, P03

Langer, M. 286

Langer, S. 379

Längle, F. 069

Lau, S. 290

Lauf, H. 268

Laufer, G. 022

Lauscher, J. C. 366

Lazariotou, M. 017

Leberfing, O. 022

Lechner, M. 050, 066, 117, 232

Lechner, W. 306, P02

Lee, W. 020, 020, 072, 125

Lehmkuhl, H. 140

Lehr, E. 146, 375

Leithner, A. P25, P26

Lerut, T. 094

Lexer, G. 335

Lichtenauer, M. 043, 044

$\mathbf{0 4 5}, 189,190$

Liebhard, B. 335

Lin, C.-H. 072, 125

Lindenmann, J. P20, P21

Linder, A. P18

Linke, G. 156

Linni, K. 322

Lippert, H. 268, 391, P22, P23, P31, P37

Lodes, U. 268

Lohberger, B. P26

Loibner-Ott, N. 304

Lucciarini, P. 103, 236, 237

Lukschal, A. P03

Lutke-Holzik, M. 165

Luz, S. 156

\section{M}

Maaß, V. 018 
Maier, H. 237

Maier, T. $\quad 170$, P24

Mair, R. 141, 142, 143

Malfertheiner, P. 391

Malliga, D. E. 073, 382

Maresch, J. 282

Margreiter, R. 020, 071, 240

Mark, W. 010, 024, 097, 111

Maroschke, F. 414

Marsoner, H. 064

Marti, L. 262

Martin, F. 011

Martinec, D. V. 158

Maruszewski, M. 122

Mathur, P. 249

Matzi, V. 230, P20, P21

May, C. 418

Mayer, F. 066, 117, 232

Mayr, P. 250, 253

Mayr, T. 098

Meier, M. 247

Meindl, M. 303

Meissnitzer, T. P07

Merkel, S. 281

Messenbäck, F. G. 108

Messner, B. 024

Mészáros, K. 087, 377, 378

Metzger, P. 092

Metzler, S. 184

Meyer, F. 268, 314, 391, P10,

P12, P13, P22, P23, P31,

P36, P37

Miah, A. P27

Mica, L. 149, P44, P45

Michaelidou, M. 037

Michlits, W. 039, 211, 252

Miesbauer, M. P33

Mika, K. 118

Mikuz, G. 196, 240

Mildner, M. 019, 043, 044, $045,189,190$

Mille, M. 175

Miller, K. 303

Mirow, L. 155

Mischinger, H. J. 068, P06, P30

Mitterbauer, A. $\quad 044,189$

Mittermair, C. 007, 009, 012,

$013,057,059,060,114$,

$115,157,221, \mathbf{2 2 4}, 225$, $226,315,390$

Mittermair, R. $\quad$ 061, 222, 299 . 300

Mitterwallner, S. 376

Mittlböck, M. 416

Moidl, R. (084, 086, 185, 380

Mönkemüller, K. 391

Moons, J. 094

Möschel, M. 363

Moser, P. 124

Mroczkowski, P. 268

Muggli, B. 341

Mühlbacher, J. 323

Mühlmann, G. 061, 222, 299, 300

Müller, A.-K. P36

Müller, B. 324

Müller, H. 087

Müller, L. 055, 081, 083, $139,144,374$

Müller, S. 081, 083

Muschweck, H. 392

Music, M. P28

\section{N}

Nafteux, P. 094

Nagel, F. 124
Nagel, W. 325

Nagele-Moser, D. 128

Nagiller, J. 184

Nasr, I. 020

Nehoda, H. 130, P01

Neidenbach, P. $\mathbf{P 0 5}$

Nemec, H. 148

Nemeth, C. 170, P24

Nesser, J. P43

Neuböck, N. P20, P21

Neuhold, N. 383

Neureiter, D. 102

Nickl, S. 044, 189, 190

Niederle, B. 419

Niel, J. P43

Niernberger, T. 095, 133, P07

Novak, A. P26

\section{O}

Oberhuber, R. $\quad$ 024, 071

Oberkanins, C. 415

Oberwalder, M. 340

Obrist, C. 007, 009, 012,

$013,057,059,060,114$,

115, 157, 221, 224, 225,

226, 315, 390

Obrist, P. 071

Obwegeser, A. A. 032

Ocvirk, J. P28

Oechs, A. P21

Oesterle, L. P45

Öfner-Velano, D. 018, 066, 096, 102, 117, 129, 230, $232,241,260,337$

Öllinger, R. 046, 074, 075, 097

Olszewski, U. 166

Omejc, M. P28

Opykan, N. 291

Oster, O. 381, P19, P42

Ott, J. 420

Ott, T. P07

Otterbein, L. E. 074

Ovcina, I. 126

Özkan, S. 186

\section{P}

Pachinger, O. 081, 083

Pandini, U. 064

Panotopoulos, J. 348

Papp, C. 211

Partri, P. 116

Parvizi, D. 257

Pau, M. 030

Paulhart, M. 036, 038

Pauschinger, M. 054

Pauzenberger, R. 323, 349

Payer, B. A. 282

Pech, M. P10

Pentsch, A. P39

Perathoner, A. 061, 090, 194, 222, 240, 299

Pereyaslov, A. 109, 245, 291, 295

Perrakis, A. 281

Peschaut, T. 293

Petersen, M. P23

Petter-Puchner, A. 052, 062 , 063, 118, 127

Pfeiffer, S. 054, 085, P41

Pichler, M. P30

Pierer, G. 349

Pierro, A. 191

Pieske, B. 187,188
Pikula, R. 323

Pilz, M. 110

Pimpl, K. 007, 009, 012, 013,

057, 059, 060, 114, 115,

157, 221, 224, 225, 226, 315, 390

Pirklbauer, K. 029

Pisarek, H. 179

Pittermann, A. 251

Pizzinini, R. 064

Placheta, E. 037

Plank, C. 040

Plank, F. 145

Pluschnig, U. 168

Podesser, B. K. 023, 043 , 045, 124, 189, 190

Poglitsch, M. 119, 258

Pokorny, H. 261

Pollak, H. 082

Pomberger, G. 246

Pomper, G. $\mathbf{0 2 3}$

Pona, I. $\quad 037,038,350$

Pones, M. 244, 289

Ponschab, M. 152

Pop, L. 170, P24

Portugaller, H. 326, 327

Porubsky, C. P20

Pöschl, G. 151

Pöschl, P. W. 029

Post, H. 187, 188

Pöstlberger, S. P33

Potapov, E. 180

Prager, G. $119,258,364$, P03

Pramhas, M. 385

Prandl, E. C. 073

Prandstetter, C. 142

Pratschke, J. 010, 020, 024, 046, 061, 071, 072, 074,

075, 093, 096, 097, 101,

103, 111, 120, 125, 222,

240, 242, 299, 300, 336,

$338,340,384,417,421$

Preldzic, L. 420

Pressl, G. 091

Presul, E. 070

Prisadov, G. P18

Profanter, C. 243

Promberger, R. 420

Prommegger, R. $\quad 384,421$

Pülzl, P. 207

Pumberger, W. 142

Puri, P. 191

Putzer, D. 421

Putz-Scheicher, K. 230

\section{R}

Raab, S. 134

Rabl, H. 095, 133, P07

Radauer, W. 207

Radda, C. T. $\quad$ P14

Rafolt, D. 128

Rancan, M. P44

Rapp, L. P37

Rasp, G. 351

Rath, T. 036, 038, 136

Ratschiller, T. P32

Rauscher, B. 415

Razek, P. 116

Rebhandl, W. 244, 247

Reck, C. A. 248

Redl, H. 052, 062, 063, 118, 127

Reich-Weinberger, S. 129, 241

Reinbacher, K. 030, 031

Reischl, M. 152

Reiter, C. 169, P24

Reiter, H. P29

Renz, O. 193

Resch, T. 075, 340, 384, 42

Ribitsch, V. 126

Ricke, J. P12

Riede, G. 024

Rieder, E. 119, 153, 158

Riedl, O. 306, 416, P02

Rieger, K. 186

Rieger, M. 032, 192

Rienmüller, R. 087

Rigler, M. Y. 073

Ring, S. 303

Rinner, B. P26

Riss, P. 419

Riss, S. 316

Ritz, J.-P. 366

Roessner, A. P23

Roka, R. 297, 298, 301, 302, 385,389

Roka-Palkovits, J. $\quad 035,040$, 049, 136

Roka-Palkovits, J. 035

Rokitansky, A. 246

Rollinger, G. 261

Rosen, H. 050

Rosenberg, R. 165

Rossmann-Tsybrovskyy, M. 073

Rothe, A. U. $\quad$ P40

Rothe, K. 290

Rouden, C. 325

Rudas, M. 168, 416

Rudin, M. 150

Russe, E. 207, 351

Russmüller, G. 029

Ruttenstock, E. M. 191

Ruttmann-Ulmer, E. 181 , 182, 183

\section{S}

Sachsenmaier, M. 284

Sachwitz, D. P12

Sahora, K. 099

Sakic, A. 181, 182, 183

Salaymeh, L. 377

Salazar, R. 165

Sames-Dolzer, E. 141, 143

Samonigg, H. P30

Sandner Kiesling, A. 186 , 230

Santarpino, G. $\quad$ 054, 085

Santer, D. 124

Sattler, S. 306, P02

Sauer, J. 285

Sautner, T. 231, 313

Saxena, A. 121, 123, 198 , 249, 293

Schachner, T. $022,145,146$ 184,375

Schaden, W. 042

Schalamon, J. 198, 293 
Schimpl, G. 148, 207, 249a, 294, P16

Schintler, M. 138, 257

Schirnhofer, J. $\quad \mathbf{0 0 7}, 009,012$ $013,057, \mathbf{0 5 9}, 060,114$ $115,157,221,224,225$. $226,315,390$

Schistek, R. 181, 182, 183

Schlager, A. 120

Schlechta, B. 122

Schmerlaib, J. 335

Schmid, K. 018

Schmid, T. 103, 112, 120,

$236,237,238, \mathbf{2 3 9}, 240,242$

Schmid, W. 053

Schmidt, J. 088

Schmidt, K. 092

Schmidt, M. $\mathbf{3 5 0}$

Schmitz, M. 129

Schneeberger, S. 020, 072 , 097, 125

Schneider, F. $\mathbf{0 5 6}$

Schnöll, J. 148, 249a

Schöb, O. P05

Schober, P. H. 198

Schöppl, S. 305

Schoppmann, A. 069

Schoppmann, S. 069, 167, 169, 364, 414

Schratt, J. 138

Schreiner, M. 329, P39

Schreiner, W. 381, P19, P42

Schrögendorfer, K. F. 136

Schubert, H. 207, 351

Schubert, S. 140

Schultes, G. 031

Schultheis, A. 383

Schultz, C. P03

Schulz, H. U. 268, P31

Schütte, K. P23

Schwabegger, A. 236, 349

Schwaller, A. P44

Schwarzl, M. 187, 188

Schweiger, M. 140, 180

Schweiger, S. 327

Schweigert, M. 286, 365, 392, P38

Sedlak, M. P40

Seebacher, G. P34

Seebacher, U. 197

Seemann, R. 029

Sellner, F. 100

Senbaklavaci, O. 089

Sergi, C. 070

Shakeri-Leidenmühler, S 258, P03

Shamiyeh, A. 220, 329, P39

Sheu, H. P33

Siercks, I. 156

Simmen, H. 149, P45

Simonitsch, K. H. 335

Singh, D. 034

Sipos, W. 045

Sirbu, H. 381, P19, P42

Sirch, J. P41

Sitzmann, G. 011

Slama, A. 053

Smolle-Jüttner, F. M. 230, P20, P21

Sodeck, G. H. 179

Sokullu, F. 252
Soleiman, A. 076

Sommerfeldt, S. 169

Sorko, K. 259

Spaun, G. O. 158

Spechtenhauser, B. 098, P32

Spendel, S. 121, 138, 257

Sperker, C. 297, 298, 301, 302, 385, 389

Stadlhuber, R. J. $\quad 286, \mathbf{3 6 5}$,

$$
\text { 392, P38 }
$$

Stanek, C. 418

Stanger, O. 376

Stangl, F. 133

Starlinger, P. 169, 170, P24

Stättner, S. 100, 135, 137, 234, 312, 413

Stauber, R. 087

Steendijk, P. 187, 188

Steger, C. 024

Steger, G. 416

Stein, H. J. 286, 365, 392, P38

Steiner, G. 152

Stepanenko, A. 180

Steringer-Mascherbauer, R. P43

Stier, A. 175

Stift, A. 284

Stifter, K. $\quad 055,139,144,374$

Stockhammer, V. 348

Stoiber, M. 119

Stojakovic, T. P30

Stopfer, J. 316, P08

Strasak, A. M. 182

Strau, G. 137,312

Stremitzer, S. 282

Stromberger, K. 075

Struller, F. M. 074

Stuendl, N. P26

Sucher, R. 072, 075, 125

Swanstrom, L. L. $\quad 153,158$

Szabo, K. P39

\section{$\mathrm{T}$}

Tabernero, J. 165

Taghavi, S. 280

Taheri, N. 179

Tamandl, D. 069, 169, 282

Tarantino, I. 156

Taspinar, H. 089

Tepeköylü, C. $\mathbf{0 4 2}$

Thalhammer, S. $\quad \mathbf{1 0 0}, 135$

Thalmann, M. 082, 179

Thomas, M. 075

Thometich, B. 023

Toller, W. 186

Tomasch, G. 128

Tomaselli, F. 006, 058

Tonninger, K. 383

Tontsch, A. 236

Trescher, K. 023, 124

Troppmair, J. 076

Truschnig-Wilders, M. 187, 188

Tschachler, E. 019, 045

Tschann, P. 363

Tscheliessnigg, K. H. 087, 126, 186, 230, 377, 378

Tsybrovskyy, O. 073
Tuchmann, A. 116

Tulzer, G. 141, 143

Turina, M. 149

Tzou, C.-H. J. 037

U

Uggowitzer, M. P07

Ugurluoglu, A. 322

Ulmer, H. $\quad 182,183$

Unger, E. 380

Untersmayr, E. P03

Uranüs, S. 073, 128

Urbanek, B. 053

\section{V}

Vahl, C. F. 089

Vallant, S. 076

van der Hoeven, J. 165

Van Raemdonck, D. 094

Vaselic, S. 348

Veit, F. H. $\quad$ 082, 179

Veits, L. 240

Velenik, V. P28

Vesely, M. 375

Vierecke, J. 180

Virnik, S. A. 028

Vischer, H. M. 328

Vogel, W. 240

Vollenweider, A. P44

von Rahden, B. 017

\section{W}

Waclawiczek, H. 102

Wagner, D. 068, 087

Wagner, H. E. 341

Waldenberger, F. 185

Waldert, J. 128

Waldstein, N. 007, 009, 012, $013,057,059,060,114$

$115,157,225,315,390$

Wallimann, H. P18

Wallner, C. 076

Wandschneider, W. 147

Wang, C. J. 042

Wang, F. S. 042

Wanner, G. A. 149, P45

Wanzar, C. 314

Wanzar, I. P36

Warschkow, R. 156

Watschinger, K. 024, 071

Wayand, W. 110, P39, P40

Weber, M. P10

Weber, T. 304

Weber-Eibel, J. 404

Wechselberger, G. 207, 351

Weder, W. 021

Weghuber, D. 303

Wegiel, B. 074

Weidinger, F. 145, 146, 184, 375

Weigel, G. 121, 138, 347

Weiss, G. 084, 086, 185
Weiß, G. 268

Weiss, H. 007, 009, 012, 013 , 057, 059, 060, 114, 115, $157,221,224,225,226$ 315,390

Weissenbacher, A. 020, 046, 075, 299

Welcker, K. P18

Wenger, R. 251

Wenzl, E. 165,363

Werba, G. 019, 043, 044 099, 189, 190

Werkgartner, G. 068

Werner, C. 149, P45

Werner, E. R. 024, 071

Wetscher, G. 090

Wex, C. P37

Whiteford, M. H. 158

Wiedemann, D. 022, 122, 146

Wiegele, A. 064

Wielandner, M. P01

Will, U. 314, P36

Wimmer, C. 056

Wimmer, G. 421

Windhager, R. 348, P25

Windhofer, C. 211

Winslet, M. P27

Wiplinger, S. 090

Wittens, C. H. 051

Witzel, K. 108

Wolf, C. 097

Wolf, G. 073, 382

Wolff, K. S. 151, 262

Wolzt, M. 074

Wunderlich, M. 259

Wutzl, A. 029

Wykypiel, H. F. 090, 240, 242

\section{Y}

Yang, S.-Y. P27

Yates, A. E. 378

Yates, A. 186

\section{Z}

Zacherl, J. 364

Zaglmair, W. 339

Zambanini, C. 093,336

Zdrojek, A. 381, P19, P42

Zeillinger, R. 415

Zelger, B. $\quad 020,125,192$

Zerz, A. 156, 227

Zheng, X. 072

Zimmermann, M. $\quad 045,189$, 190

Zimrin, D. 146, 375

Zirngast, B. $\quad 087,186,377$. 378

Zitt, M. 093, 096, 229, 336, 338, 417

Zlabinger, G. 247

Zöch, G. 208

Zosso, C. 150

Zuckermann, A. O. 023

Medieninhaber und Herausgeber: Springer-Verlag GmbH, Sachsenplatz 4-6, 1201 Wien, Österreich. - Datenkonvertierung und Umbruch

Thomson Press (India) Ltd., Chennai; Druck: Holzhausen Druck GmbH, 1140 Wien Österreich. - Verlagsort: Wien. - Herstellungsort: Wien. 